\title{
Forcing e Regularidade na Reta Real
}

\author{
Michel Fernandes Gaspar \\ DISSERTAÇÃO APRESENTADA \\ $\mathrm{AO}$ \\ Instituto De Matemática e Estatística \\ DA \\ UNIVERSIDADE DE SÃo PAULO \\ PARA \\ OBTENÇÃO DO TÍTULO \\ $\mathrm{DE}$ \\ Mestre em CiÊnCIAS
}

Programa: Matemática

Orientador: Prof. Dr. Rogério Augusto dos Santos Fajardo

Durante o desenvolvimento deste trabalho o autor recebeu auxílio financeiro do CNPq

São Paulo, março de 2018 


\section{Forcing e Regularidade na Reta Real}

Esta é a versão original da dissertação elaborada pelo candidato Michel Fernandes Gaspar, tal como submetida à Comissão Julgadora. 


\section{Forcing e Regularidade na Reta Real}

Esta versão da dissertação contém as correções e alterações sugeridas pela Comissão Julgadora durante a defesa da versão original do trabalho, realizada em 05/03/2018. Uma cópia da versão original está disponível no Instituto de Matemática e Estatística da Universidade de São Paulo.

Comissão Julgadora:

- Prof. Dr. Rogério Augusto dos Santos Fajardo (orientador) - IME-USP

- Prof. Dr. Leandro Fiorini Aurichi - ICMC-USP

- Prof. Dr. Ana Carolina Boero - CMCC-UFABC 


\section{Agradecimentos}

Primeiramente, agradeço ao meu orientador, Professor Dr. Rogério Augusto dos Santos Fajardo, por todos os anos de dedicação como orientador. Segundo, agradeço aos membros da banca pelas correções e valiosos comentários em prol da melhora deste trabalho.

Agradeço também à funcionária Ana Paula, por seu excelente trabalho na secretaria da pósgraduação.

Agradeço aos meus colegas do IME, especialmente à Cláudia Correa, que se disponibilizou a assistir à prévia da minha defesa; ao Gabriel Fernandes, com quem pude discutir diversos dos problemas deste trabalho; e ao Vinícius Rodrigues, com que tirei diversas dúvidas sobre teoria dos conjuntos.

Agradeço à minha mãe, Rosa Emília Fernandes da Silva Bonfim, por todo o amor, e por todo o incentivo para que eu seguisse meu sonho de estudar Matemática.

Agradeço a todos os meus amigos por toda a alegria que passamos juntos, por todo o suporte e por toda a compreensão. Gostaria de agradecer especialmente à Ana Flávia Gindro e ao Lucas Felipe Iria, por estarem ao meu lado nos eventos mais importantes da minha vida; e ao meu querido amigo Tales Rodrigues, com quem morei nos últimos três anos e cujo suporte nesse período foi imprescindível para o bom andamento do meu mestrado.

Agradeço ao Arthur Bonfá Fernandes, por todo o amor e carinho.

Por fim, agradeço ao CNPq, pelo auxílio financeiro que tornou este trabalho possível. 


\section{Resumo}

Gaspar, M. F. Forcing e Regularidade na Reta Real. 2018. 118 f. Dissertação (Mestrado) Instituto de Matemática e Estatística, Universidade de São Paulo, São Paulo, 2010.

O estudo das propriedades de regularidade na reta real é tão antigo quanto o surgimento da teoria dos conjuntos no final do século XIX. Essas propriedades indicam bom comportamento para subconjuntos da reta real, sendo os exemplos mais proeminentes a propriedade do conjunto perfeito, a Lebesgue mensurabilidade e a Baire mensurabilidade. Neste trabalho outras propriedades de regularidade são exploradas, como a propriedade de Ramsey, a propriedade doughnut, a Marczewski mensurabilidade, a Miller mensurabilidade, a Laver mensurabilidade, dentre outras. A relação que existe entre propriedades de regularidade e forcing é conhecida desde a década de 70 com os trabalhos de Robert Solovay, que, por exemplo, construiu um modelo de teoria dos conjuntos onde todo subconjunto da reta real é Lebesgue mensurável, Baire mensurável e tem a propriedade do conjunto perfeito. Todas essas propriedades de regularidade são capturadas em uma definição geral recorrendo à poderosa técnica do forcing idealizado, introduzida e explorada por Jindrich Zapletal em 2004. O principal estudo sistemático das propriedades de regularidade via forcing idealizado foi feito por Yurii Khomskii em 2012 em sua tese de doutorado. O resultado de Solovay mencionado acima é provado nesse contexto geral de regularidade. Também são exploradas caracterizações para a regularidade dos conjuntos no segundo nível da hierarquia projetiva via forcing sobre L. Para a maioria dos assuntos abordados é dada alguma nota histórica.

Palavras-chave: Regularidade na reta real, Forcing, Forcing idealizado, Hierarquia projetiva. 


\section{Abstract}

Gaspar, M. F. Forcing and Regularity in the Real Line. 2018. 118 f. Dissertação (Mestrado) - Instituto de Matemática e Estatística, Universidade de São Paulo, São Paulo, 2018.

The study of the regularity properties in the real line is as old as the beginning of set theory at the end of the 19th century. These properties indicate well behavior for subsets of the real line, being the Lebesgue measurability, Baire measurability and perfect set properties the most prominent examples. In this work other regularity properties are explored, such as the Ramsey property, the doughnut property, the Marczewski measurability, the Miller measurability, the Laver measurability, among others. The relationship between regularity properties and forcing is known since the 70's with the work of Robert Solovay, who, for example, constructed a model of set theory in which every subset of the real line is Lebesgue measurable, Baire measurable, and has the perfect set property. All of theses regularity properties are captured by a general definition making use of the powerful technique of idealized forcing, introduced by Jindrich Zapletal in 2008. The main systematic study of regularity properties via idealized forcing was done by Yurii Khomskii in 2012 in his $\mathrm{Ph} . \mathrm{D}$ dissertation. The result of Solovay mentioned above is proved in this general framework. Characterization results for regularity properties of the sets in the second level of the projective hierarchy via forcing over L are also explored. Some historical notes are provided for most of the addressed subjects.

Keywords: Regularity in the real line, Forcing, Idealized Forcing, Projective hierarchy. 


\section{Sumário}

1 Introdução $\quad \mathbf{1}$

1.1 Considerações iniciais . . . . . . . . . . . . . . . . . . . . 1

1.2 O Problema do Continuum e a primeira noção de regularidade . . . . . . . . . . . 1

1.3 A emergência da teoria axiomática dos conjuntos . . . . . . . . . . . . . 3

1.4 O estudo da regularidade ao longo de hierarquias de complexidade . . . . . . . . . 10

1.5 Em L há contraexemplos para a regularidade dos conjuntos projetivos . . . . . . . . 18

2 Teoria descritiva dos conjuntos $\quad 23$

2.1 Como codificar borelianos usando números reais . . . . . . . . . . . . . . . . 23

2.2 Representação arbórea dos conjuntos analíticos . . . . . . . . . . . . . . 26

2.3 Representação arbórea de Shoenfield dos conjuntos $\boldsymbol{\Sigma}_{2}^{1} \ldots \ldots \ldots$. . . . . . . . 31

2.4 Como provar teoremas sobre borelianos usando jogos . . . . . . . . . . . . . . . 34

$\begin{array}{lll}3 & \text { Teoria geral do forcing } & 39\end{array}$

3.1 Forcing: uma abordagem heurística . . . . . . . . . . . . . . . . 39

3.2 Tecnologia do forcing . . . . . . . . . . . . . . . . . . . . . . . 48

3.3 Um mínimo sobre forcing iterado . . . . . . . . . . . . . . . . . 55

4 Dualidade entre regularidade e forcing $\quad 61$

4.1 Plano geral . . . . . . . . . . . . . . . . . . . . . . . . 6 . 61

4.2 O forcing aleatório foi o primeiro forcing idealizado . . . . . . . . . . . . . 62

4.3 Marczewski mensurabilidade e o forcing de Sacks . . . . . . . . . . . . . . . . 66

4.4 E se trocarmos árvores perfeitas por superperfeitas? . . . . . . . . . . . . . . . 69

4.5 Uma noção de regularidade advinda da conjectura de Borel . . . . . . . . . . . . . . 72

4.6 Uma noção de regularidade relacionada com colorações de subconjuntos de $\omega$. . . . 75

4.7 Fazendo doughnuts com árvores de Silver f . . . . . . . . . . . . . . . . 78

$\begin{array}{llr}5 & \text { Regularidade idealizada } & \mathbf{8 3}\end{array}$

5.1 Propriedades gerais do forcing idealizado . . . . . . . . . . . . . 83

5.2 Mensurabilidade obtida colapsando um cardinal inacessível . . . . . . . . . . . . . 88

5.3 Mensurabilidade dos conjuntos $\boldsymbol{\Delta}_{2}^{1}$ e $\boldsymbol{\Sigma}_{2}^{1}$ via forcing sobre L . . . . . . . . . . . . . 93

5.4 Considerações finais . . . . . . . . . . . . . . . . . . . . . . 100

$\begin{array}{ll}\text { Referências Bibliográficas } & 103\end{array}$ 


\section{Capítulo 1}

\section{Introdução}

\subsection{Considerações iniciais}

Com relação aos requisitos, é assumida familiaridade com teoria dos conjuntos, lógica matemática, topologia geral e um mínimo em teoria da medida. Além desses assuntos, também esperamos que o leitor tenha visto alguma vez as definições de álgebra de Boole e de álgebra de Boole completa. Usamos a linguagem $\mathcal{L}=\left\{\leq, \vee, \wedge,{ }^{\prime}, 0,1\right\}$ com os significados usuais para a teoria das álgebras de Boole.

Faremos uma convenção importante: para a maioria dos matemáticos, o conjunto dos números reais é denotado por $\mathbb{R}$, e é caracterizado como o único corpo ordenado completo a menos de isomorfismo, para certas operações de soma e produto. O conjunto dos reais com a topologia usual é conexo, não-compacto, $\sigma$-compacto, etc. Porém, para os teoristas dos conjuntos e para nós, os reais podem ser qualquer um dos seguintes espaços:

$$
\omega^{\omega}, 2^{\omega},[\omega]^{\omega} .
$$

Frequentemente precisaremos trabalhar com um espaço ou outro, na hora de estudar propriedades de regularidade. Se $X$ é 2 ou $\omega$, então para cada $s \in X^{<\omega}$, definimos

$$
\mathcal{O}_{s} \doteq\left\{f \in X^{\omega} \mid s \subseteq f\right\} .
$$

Assim, $\left\{\mathcal{O}_{s} \mid s \in X^{<\omega}\right\}$ forma base para uma topologia em $X^{\omega}$, que nada mais é do que a topologia produto, onde $X$ e $\omega$ são equipados com a topologia discreta. Consideraremos também os espaços $\left(\omega^{\omega}\right)^{k}$ e $\left(2^{\omega}\right)^{k}$, para $k>1$, tendo o significado usual. Trabalharemos exclusivamente com esses espaços, fazendo toda a teoria geral para $\omega^{\omega}$ (para $2^{\omega}$ é facilmente adaptável), raramente fazendo referência a $\mathbb{R}$ "de verdade". Note que há diferenças topológicas não negligenciáveis entre esses espaços. Por exemplo, $2^{\omega}$ é compacto, $\omega^{\omega}$ não é compacto, nem $\sigma$-compacto, enquanto ambos são 0-dimensionais, e portanto desconexos. No entanto, propriedades clássicas relativas à medida de Lebesgue, categoria de Baire, e propriedade do conjunto perfeito podem ser transportadas usando certas funções "boas" (veja [Lev02], Capítulo VII). Outras propriedades só fazem sentido em um espaço ou outro. Por essas e outras razões, alguns autores preferem o nome regularidade do continuum, ao invés de "regularidade na reta real".

\subsection{O Problema do Continuum e a primeira noção de regularidade}

Sempre ouvimos que a teoria dos conjuntos surgiu com a investigação de Cantor acerca das séries trigonométricas. O matemático Heine em 1869 propôs para o jovem Cantor o seguinte problema sobre a unicidade da representação das séries trigonométricas: se $a_{0} / 2+\sum_{n=1}^{\infty}\left(a_{n} \cos (n x)+\right.$ $\left.b_{n} \operatorname{sen}(n x)\right)=0$ para todos $x, a_{n}, b_{n} \in \mathbb{R}$, é verdade que $a_{n}=b_{n}=0$ para todos $n \in \mathbb{N}$ ? Esse problema surgiu dos trabalhos de Heine e de Riemann; e sua solução afirmativa foi dada por Cantor em 1870. A fim de generalizar seu resultado sobre séries trigonométricas, Cantor em 1872 [Can72] 
enfraqueceu a hipótese de que a série converge em todos os pontos, da seguinte forma: para uma coleção $P$ de números reais, Cantor definiu $P^{\prime}$ o conjunto dos pontos de acumulação de $P$, e então iterou essa operação sobre $P$ definindo $P^{(n)}$ o resultado de $n$ iterações da aplicação $P \mapsto P^{\prime}$.

Assim, Cantor provou que se $\frac{a_{0}}{2}+\sum_{n=1}^{\infty}\left(a_{n} \cos (n x)+b_{n} \operatorname{sen}(n x)\right)=0$ para todos $a_{n}, b_{n} \in \mathbb{R}$ e $x \in \mathbb{R} \backslash P$, onde $P$ é uma coleção de reais tal que $P^{(m)}=\emptyset$ para algum $m \in \mathbb{N}$, então $a_{n}=b_{n}=0$ para todo $n \in \mathbb{N}$. Indo mais além, Cantor já considerava iterações infinitas de sua operação $P^{\prime}$, mais ou menos da seguinte forma:

- $P^{(\infty)}=\bigcap_{n \in \mathbb{N}} P^{(n)}$.

- $P^{(\infty+1)}=P^{(\infty)^{\prime}}$.

- $P^{(\infty+2)}, \ldots, P^{(\infty \cdot 2)}, \ldots, P^{(\infty \cdot \infty)}$, etc.

Eventualmente, Cantor começou a investigar coleções infinitas de números reais e enumerações infinitas, e isso o levou aos conceitos básicos sobre o continuum e à formulação dos números transfinitos. Em dezembro de 1873 Cantor estabeleceu a não-enumerabilidade do conjunto dos números reais e em 1874 [Can74] estabeleceu a enumerabilidade para os números reais algébricos, fornecendo uma nova prova de que existem números transcendentais. Em 1877 [Can77] Cantor voltou sua atenção para correspondências bijetoras motivado pelo fato de que em seu trabalho anterior [Can74] ele mostrou a inexistência de uma correspondência bijetora entre $\mathbb{N}$ e $\mathbb{R}$. Cantor, entretanto, só conseguiu encontrar duas potências de infinitos: enumerável e continuum. Assim, Cantor conjecturou:

"Todo conjunto infinito de reais ou é enumerável ou tem a potência do continuum."

Esse problema é conhecido como Hipótese do Continuum $(\mathrm{CH})$. Pode-se dizer que a teoria dos conjuntos teve seu início numa tentativa de solucionar o Problema do Continuum.

Em 1883 [Can83] Cantor introduziu os números ordinais e o conceito de boa ordem, e trocou o uso do símbolo $\infty$ por $\omega$, a última letra do alfabeto grego. Fundamental nesse trabalho de Cantor foi a sua formulação do Princípio da Boa Ordem:

"É sempre possível colocar qualquer conjunto bem-definido na forma de um conjunto bem-ordenado."

Cantor então tomou dois caminhos na tentativa de resolver o Problema do Continuum:

1. A abordagem através da potência.

2. A abordagem via conjuntos definíveis de reais.

Na primeira abordagem, Cantor definiu o que hoje conhecemos como $\omega$ (ou $\aleph_{0}$ ), o conjunto dos números naturais, $\omega_{1}$ (respec. $\aleph_{1}$ ) o conjunto dos ordinais infinitos enumeráveis e $2^{\omega}$ como sendo o continuum. Com essa nomenclatura, provar a Hipótese do Continuum é provar que $2^{\omega}$ e $\omega_{1}$ são equipotentes, que é o que Cantor gostaria de ter feito. Essa ideia não deu frutos, sendo o principal problema a impossibilidade de Cantor estabelecer diretamente uma boa-ordem para os reais, a partir da boa ordem para $\omega$.

$\mathrm{Na}$ segunda abordagem, Cantor voltou a considerar iterações infinitas de sua operação $P^{\prime}$, usada em seu trabalho sobre séries trigonométricas. Cantor utilizou o conceito de conjunto perfeito (i.e., um conjunto $P$ tal que $P=P^{\prime}$ ), provando que todo conjunto fechado é a união de um conjunto enumerável com um conjunto perfeito. Como Cantor havia provado já em trabalhos anteriores que todo conjunto perfeito tem a cardinalidade do continuum, Cantor então havia estabelecido a Hipótese do Continuum para os conjuntos fechados. Assim, o problema estaria resolvido se Cantor provasse que existe algum conjunto fechado de cardinalidade $\aleph_{1}$. 
Definição 1.2.1. Seja $A \subseteq \omega^{\omega}$. Dizemos que $A$ é perfeito se é não-vazio, fechado e não possui pontos isolados. Isto é, $A^{\prime}=A$.

Teorema 1.2.2 (Bendixson [Ben83], Cantor [Can84]).

1. Para todo conjunto fechado $F \subseteq \omega^{\omega}$, existe um conjunto perfeito $P \subseteq F$ tal que $F \backslash P$ é enumerável.

2. Todo conjunto perfeito tem a cardinalidade do continuum.

Definição 1.2.3. Seja $A \subseteq \omega^{\omega}$. Dizemos que A tem a propriedade do conjunto perfeito se $A$ é enumerável ou se existe $P \subseteq A$ um subconjunto perfeito.

Uma breve digressão: o conjunto de todos os subconjuntos enumeráveis de $\omega^{\omega}$ forma uma $\sigma$-ideal em $\omega^{\omega}$; apenas por razões notacionais, introduzimos a seguinte definição.

Definição 1.2.4. O $\sigma$-ideal dos conjuntos enumeráveis de reais é denotado por ctbl.

Nos anos seguintes, sempre gastando esforços para solucionar o Problema do Continuum, Cantor desenvolveu sua teoria de aritmética para números ordinais (como um caso particular da aritmética para tipos de ordens) e cardinais. Em [Can91] Cantor deu seu famoso argumento de diagonalização, provando que para qualquer conjunto $M$ o conjunto das funções de $M$ em um conjunto de dois elementos tem a cardinalidade maior do que a de $M$, argumentando pela primeira vez explicitamente que existe potência acima do continuum e, mais geralmente, que não existe uma potência maior que todas as outras. Ficou em aberto, porém, seu Princípio de Boa Ordem, que seria essencial para enquadrar todos os conjuntos nessa escala transfinita que Cantor havia criado. Mais tarde Zermelo resolveria o problema da boa ordenação de todos os conjuntos.

\subsection{A emergência da teoria axiomática dos conjuntos}

Zermelo começou a investigar a teoria dos conjuntos de Cantor em Göttingen por influência de Hilbert. Zermelo em [Zer04] formulou o que ele chamou de Axioma da Escolha (AC), e com isto, estabeleceu o seu Teorema da Boa-Ordenação: "Todo conjunto pode ser bem ordenado". Entretanto, seu axioma na época já causou muita controvérsia, pois se tratava de uma função arbitrária, que fazia uma escolha simultânea, ao invés de escolhas sucessivas. Tratava-se de uma extensão da noção de função, já antecipada por Cantor.

Devido às críticas da época, Zermelo em resposta publicou, em 1908 ([Zer08]), uma axiomatização para a teoria dos conjuntos, a fim de justificar sua prova específica do Princípio da BoaOrdenação. Hoje conhecemos este sistema como ZFC, mas para chegar na forma como o conhecemos hoje, muitas alterações foram feitas ao longo dos anos.

O sistema de Zermelo era uma variação dos axiomas que conhecemos hoje como Extensão, Vazio, Par, União, Partes, Infinito, Escolha e Separação. Vale mencionar que Zermelo propôs o Axioma da Escolha a fim de formalizar a sua prova do Princípio da Boa Ordenação ([Zer07]). O sistema de Zermelo também separava os conjuntos dos elementos que não eram conjuntos. Isto é, além do símbolo relacional binário primitivo $\in$, o sistema também tinha um símbolo relacional unário que dizia quando um objeto era um conjunto. Os elementos que não eram conjuntos eram ditos urelementos.

Fraenkel teve como contribuição principal para o sistema de Zermelo o acréscimo do Axioma da Substituição, e as provas da independência dos Axiomas da Extensão, Separação e Escolha, relativamente à teoria de Zermelo, que continha urelementos. Em 1922 [Fra22] Fraenkel enunciou o Axioma da Substituição da seguinte forma: se $M$ é um conjunto e se cada membro de $M$ for substituído por um conjunto ou urelemento, então $M$ é transformado num conjunto. Quanto à independência do Axioma da Escolha, é importante ressaltar que a prova de Fraenkel usou de forma indispensável os urelementos do sistema de Zermelo, e não respondia, por exemplo, quando valiam instâncias do axioma da escolha para conjuntos "ordinários", como os reais. Apesar disto, 
Fraenkel introduziu técnicas de simetria e permutação essencialmente da mesma forma que Cohen faria para provar a independência do Axioma da Escolha, na teoria dos conjuntos sem urelementos, mais de 40 anos depois.

Além de Fraenkel, muitos trabalhos em modelos para teorias de conjuntos foram influenciados por Skolem. Skolem formulou a teoria de conjuntos dentro da lógica de primeira ordem transformando o Axioma da Separação na verdade em um esquema de axiomas [Sko22]. Skolem, porém, não achava a teoria dos conjuntos adequada para fundamentar a matemática. Influenciado por esta concepção, ele aplicou ao sistema de Zermelo o que conhecemos hoje como Teorema de LöwenheimSkolem para mostrar que a teoria de Zermelo não era categorial: se $\mathfrak{B}$ é um modelo para os axiomas de Zermelo com o Axioma da Substituição, então existe um modelo enumerável $\mathfrak{B}^{\prime}$ para os axiomas de Zermelo mais Substituição. Skolem também observou que seria sempre possível obter subestruturas sem urelementos e com somente conjuntos bem-fundados [Sko22]. A não-categoricidade do sistema de Zermelo (isto é, a impossibilidade de obter um domínio único para a teoria dos conjuntos) levou Skolem a fazer considerações sobre modelos enumeráveis para a teoria dos conjuntos que mais tarde seriam centrais no desenvolvimento do Forcing: ele observou que talvez fosse possível começar com um modelo enumerável para os axiomas de Zermelo e adicionar um conjunto de naturais de modo que a estrutura obtida ainda seria um modelo. Ele escreveu em uma nota de rodapé:

"Como os axiomas de Zermelo não determinam unicamente o domínio $\mathfrak{B}$, é muito improvável que todos os problemas de cardinalidade sejam decidíveis em termos desses axiomas. Por exemplo, é bem provável que o que é chamado de Problema do Continuum, a saber, se $2^{\aleph_{0}}$ é maior que $\aleph_{1}$ ou se é igual, não seja solúvel nesse sentido; nada é necessariamente é decidível sobre isso. A situação deve ser exatamente a mesma que no seguinte caso: um corpo comutativo não específico é dado e nos pergutamos se este contém um elemento $x$ tal que $x^{2}=2$. Isso não é determinado pois o domínio não é único."

[Sko22]

John von Neumann na tentativa de obter uma axiomatização categorial para a teoria dos conjuntos, eliminou os urelementos do sistema de Zermelo e introduziu o Axioma da Fundação [VN23]. Ele também mostrou que se o seu conjunto inicial de axiomas é consistente então é consistente também esse conjunto de axiomas junto com o seu Axioma da Fundação [VN29]. No entanto, influenciado pelo Teorema de Löwenheim-Skolem, ele suspeitava que qualquer teoria com modelo infinito não poderia ser axiomatizada categorialmente. Alfred Tarski (1901-1983) confirmou esta suspeita (em apêndice [Sko34]) mostrando que toda teoria de primeira ordem sem modelo finito possui modelo não-enumerável. E isto, somado ao Teorema de Löwenheim-Skolem, encerrou com a possibilidade de axiomatizar categorialmente a teoria dos conjuntos em lógica de primeira ordem.

A seguir listamos, em linguagem moderna, os axiomas que hoje conhecemos como $Z F C$ (ZermeloFraenkel-Choice):

Definição 1.3.1. A linguagem da teoria dos conjuntos é definida por $\mathcal{L}_{\epsilon} \doteq\{\in\}$, e os axiomas ZFC são enunciados na seguinte lista (infinita):

\section{Axioma da Extensão.}

$$
\forall x \forall y \forall z(x=y \leftrightarrow(z \in x \leftrightarrow z \in y)) .
$$

\section{Axioma da Fundação.}

$$
\forall x(x \neq \emptyset \rightarrow \exists y(y \in x \wedge \neg \exists z(z \in x \wedge z \in y))) .
$$

\section{Axioma do Par.}

$$
\forall x \forall y \exists z(x \in z \wedge y \in z) .
$$


4. Axioma da União.

$$
\forall x \exists y \forall z(z \in y \leftrightarrow \exists w(z \in w \wedge w \in x)) .
$$

5. Axioma das Partes.

$$
\forall x \exists y \forall z(z \in y \leftrightarrow z \subseteq x)
$$

6. Axioma do Infinito.

$$
\exists x(\emptyset \in x \wedge \forall y(y \in x \rightarrow S(y) \in x)) .
$$

7. Esquema de Axiomas da Separação. Para cada $\mathcal{L}_{\in}$-fórmula $\varphi$ em que y não ocorre livre, é um axioma:

$$
\forall x \exists y \forall z(z \in y \leftrightarrow z \in x \wedge \varphi) .
$$

8. Esquema de Axiomas da Substituição. Para cada $\mathcal{L}_{\in}$-fórmula $\varphi$ em que $Z$ não ocorre livre, é um axioma:

$$
\forall x(\forall y(y \in x \rightarrow \exists ! z \varphi) \rightarrow \exists Z \forall y(y \in x \rightarrow \exists z(z \in Z \wedge \varphi))) .
$$

9. Axioma da Escolha (AC).

$$
\forall x(\emptyset \notin x \wedge \forall y \forall z(y \neq z \rightarrow y \cap z=\emptyset) \rightarrow \exists C \forall y(y \in x \rightarrow \exists z(C \cap y=\{z\}))) .
$$

A seguinte tabela resume as seguintes subteorias:

\begin{tabular}{|l|l|}
\hline $\mathrm{ZFC}$ & Axiomas 1-9 \\
$\mathrm{ZF}$ & Axiomas 1-8 \\
$\mathrm{Z}$ & Axiomas 1-7 \\
$\mathrm{ZC}$ & Axiomas de $\mathrm{Z}+9$ \\
$T-\mathrm{P}$ & T sem o Axioma 5 \\
$T^{-}$ & T sem o Axioma 2 \\
$T-\operatorname{Inf}$ & T sem o Axioma 6 \\
\hline
\end{tabular}

Onde $T$ pode ser ZFC, ZF, Z ou ZC.

Vamos formalizar a ideia de "modelos para ZFC", que já foi mencionada anteriormente sob o nome domínio. A primeira coisa que devemos fazer é lidar com os conceitos de relativização e satisfação. Como é assumida uma certa familiaridade com lógica matemática e teoria dos conjuntos, faremos apenas uma breve revisão, dando ênfase apenas a alguns detalhes importantes.

Classe, para nós, significa classe definivel, de modo que é sempre possível falar dessa classe em ZF ou ZFC. Isso quer dizer que estamos considerando apenas classes do tipo

$$
\mathrm{M} \doteq\left\{x \mid \varphi\left(x, x_{1}, \ldots, x_{n}\right)\right\}
$$

para alguma $\mathcal{L}_{\epsilon}$-fórmula $\varphi\left(v_{0}, v_{1}, \ldots, v_{n}\right)$ e $x_{1}, \ldots, x_{n}$. Assim, $x \in \mathrm{M}$ é apenas uma notação que importamos da teoria dos conjuntos, para facilitar o raciocínio.

Definição 1.3.2. Sejam $\varphi$ uma $\mathcal{L}_{\in}$-fórmula, $\mathrm{M}$ uma classe e $E$ uma relação binária em $\mathrm{M}$. A relativização de $\varphi$ à estrutura $(\mathrm{M}, E)$ é a fórmula $\varphi^{\mathrm{M}, E}$ definida recursivamente na complexidade de $\varphi$ : 
- $S e \varphi$ é $x \in y:(x \in y)^{\mathrm{M}, E} \leftrightarrow x E y$

- $\operatorname{Se} \varphi$ é $x=y:(x=y)^{\mathrm{M}, E} \leftrightarrow x=y$

- $S e \varphi \dot{e} \neg \psi:(\neg \psi)^{\mathrm{M}, E} \leftrightarrow \neg \psi^{\mathrm{M}, E}$

- $S e \varphi$ é $\psi \wedge \chi:(\psi \wedge \chi)^{\mathrm{M}, E} \leftrightarrow \psi^{\mathrm{M}, E} \wedge \chi^{\mathrm{M}, E}$

- $S e \varphi e^{\exists} \exists x \psi:(\exists x \psi)^{\mathrm{M}, E} \leftrightarrow \exists x \in \mathrm{M} \psi^{\mathrm{M}, E}$

Às vezes escreveremos também $(\mathrm{M}, E) \models \varphi((\mathrm{M}, E)$ satisfaz $\varphi)$ ao invés de $\varphi^{\mathrm{M}, E}$. Escreveremos $\varphi^{\mathrm{M}}$ ou $\mathrm{M}=\varphi$ quando $E=\epsilon$.

Mais geralmente, para uma linguagem $\mathcal{L}$ qualquer, $\mathcal{M}$ uma $\mathcal{L}$-estrutura, $\varphi\left(v_{1}, \ldots, v_{n}\right)$ uma $\mathcal{L}$ fórmula e $x_{1}, \ldots, x_{n}$ elementos do domínio de $\mathcal{M}$, é possível definir a relação de satisfação $\models$, que depende de $\mathcal{M}$ e $\varphi$, recursivamente na complexidade das fórmulas, de modo que $\mathcal{M} \models \varphi\left(x_{1}, \ldots, x_{n}\right)$ indica que a fórmula $\varphi\left(v_{1}, \ldots, v_{n}\right)$ é verdadeira na estrutura $\mathcal{M}$, com atribuição de variáveis $x_{1}, \ldots, x_{n}$.

Agora faremos uma breve discussão sobre a relação de satisfação em ZFC. Primeiramente, da mesma forma que existe diferença entre os números naturais da metateoria e da teoria, existe diferença entre as fórmulas como objetos da metateoria e as fórmulas da teoria. Em se tratando de teoria dos conjuntos, a metateoria é usualmente a aritmética primitiva recursiva, PRA, e a teoria é ZF ou ZFC. Considere a teoria ZF(C) que temporariamente denota ZF ou ZFC. ZF(C) é uma teoria capaz de codificar PRA e os números naturais dentro de si mesma. Esse processo pode ser feito através de uma aritmetização da sintaxe, como foi feito por Gödel (falaremos mais disso na Seção 1.5), em que cada fórmula $\varphi$ corresponde a um único número natural, denotado por $\ulcorner\varphi\urcorner$. Em $\mathrm{ZF}(\mathrm{C})$, considere o conjunto $F$ orm das $\mathcal{L}_{\epsilon}$-fórmulas (i.e., se $\varphi$ é uma fórmula, $\ulcorner\varphi\urcorner \in$ Form).

Lema 1.3.3. Sejam $M$ um conjunto, $E$ uma relação binária em $M, \varphi\left(v_{1}, \ldots, v_{n}\right)$ uma $\mathcal{L}_{\in}$-fórmula e $x_{1}, \ldots, x_{n} \in$ M. Então

$$
\varphi^{M, E}\left(x_{1}, \ldots, x_{n}\right) \leftrightarrow(M, E) \models\ulcorner\varphi\urcorner\left(x_{1}, \ldots, x_{n}\right) .
$$

Isso significa que para o caso em que $M$ é um conjunto, a noção de relativização e de satisfação são a mesma coisa. O que de certa forma quer dizer que podemos refletir as $\mathcal{L}_{\in}$-fórmulas para dentro das $\mathcal{L}_{\epsilon}$-estruturas. Uma vez que fixamos um conjunto $M$ e uma $\mathcal{L}_{\epsilon}$-fórmula $\varphi$, a relação $\models$ é uma $\mathcal{L}_{\in}$-fórmula $\models\left(v_{0}, v_{1}\right)$ e definimos $\models(M,\ulcorner\varphi\urcorner)$. Entretanto, para o caso em que $\varphi \in F$ orm, i.e., uma fórmula dentro de $\mathrm{ZF}(\mathrm{C})$, a noção de relativização não está definida, e para o caso em que $M$ é uma classe própria, a noção de satisfação não está definida.

Definição 1.3.4. Uma noção de verdade é uma $\mathcal{L}_{\in}$-fórmula True $(v)$ com as seguintes propriedades:

- $\forall x(\operatorname{True}(x) \rightarrow x \in \omega)$

- Se $\sigma$ é uma sentença, então $\sigma \leftrightarrow \operatorname{True}(\ulcorner\sigma\urcorner)$.

Teorema 1.3.5 (Teorema da Indefinibilidade da Verdade). Não existe uma noção de verdade.

Demonstração. Suponha que existe True $(v)$ uma noção de verdade. Seja $\varphi_{0}, \ldots, \varphi_{n}$ uma enumeração de todas as $\mathcal{L}_{\in}$-fórmulas com uma variável livre e considere $\psi(x)$ a fórmula

$$
x \in \omega \wedge \neg \operatorname{True}\left(\left\ulcorner\varphi_{x}(x)\right\urcorner\right) .
$$

Existe um número natural $k$ tal que $\psi$ é $\varphi_{k}$. Seja $\sigma$ a sentença $\psi(k)$ (i.e., $\varphi_{k}(k)$ ). Então

$$
\sigma \leftrightarrow \psi(k) \leftrightarrow \neg \operatorname{True}\left(\left\ulcorner\varphi_{k}(k)\right\urcorner\right) \leftrightarrow \neg \operatorname{True}(\ulcorner\sigma\urcorner) .
$$

Isso contradiz a definição de noção de verdade. 
Para resolver esse problemas, introduziremos a primeira (das muitas) hierarquia desse texto: a hierarquia de Levy. Considere $\varphi$ uma $\mathcal{L}_{\in}$-fórmula. Dizemos que $\varphi$ tem quantificadores limitados se todo quantificador de $\varphi$ é da forma $\forall x \in y$ ou $\exists x \in y$. Convencionaremos essa nomenclatura sem uma definição precisa.

Definição 1.3.6. Seja $n<\omega$. Definimos a hierarquia dos conjuntos $\Sigma_{n}$, $\Pi_{n}$ e $\Delta_{n}$ por recursão finita da seguinte forma:

- $\varphi \in \Sigma_{0}$ se, e somente se, $\varphi \in \Pi_{0}$ se, e somente se, $\varphi$ é uma $\mathcal{L}_{\in}$-fórmulas com quantificadores limitados.

- $\varphi \in \Pi_{n}$ se, e somente se, $\neg \varphi \in \Sigma_{n}$

- $\varphi \in \Sigma_{n+1}$ se, e somente se, $\varphi$ é da forma $\exists v \psi$ e $\psi \in \Pi_{n}$.

- $\Delta_{n} \doteq \Sigma_{n} \cap \Pi_{n}$.

Essa hierarquia é dita hierarquia de Levy. Dizemos que $\varphi \in \Sigma_{n}^{\mathrm{ZF}}$ se existe uma fórmula $\varphi^{\prime}$ tal que $\mathrm{ZF} \vdash \varphi \leftrightarrow \varphi^{\prime}$.

A teoria ZF classifica todas as $\mathcal{L}_{\epsilon}$-fórmulas na hierarquia de Levy. Sequências de quantificadores iguais podem ser contraídos usando sucessivas aplicações do Axioma do Par, e quantificadores limitados não aumentam a complexidade de uma fórmula. A primeira observação sobre a hierarquia de Levy é que fórmulas com complexidade baixa são absolutas. Tornaremos essa noção precisa.

Definição 1.3.7. Sejam M uma classe e $\varphi\left(v_{1}, \ldots, v_{n}\right)$ uma $\mathcal{L}_{\in}$-fórmula. Dizemos que $\varphi$ é absoluta para $\mathrm{M}$ se para todos $x_{1}, \ldots, x_{n} \in \mathrm{M}$,

$$
\mathrm{ZF} \vdash \varphi^{\mathrm{M}}\left(x_{1}, \ldots, x_{n}\right) \leftrightarrow \varphi\left(x_{1}, \ldots, x_{n}\right) .
$$

Verificar que certas noções são absolutas para modelos transitivos de ZF é uma prática rotineira e pode ser encontrada em livros clássicos como [Kun14]. Convencionamos que, nos contextos em que absolutividade for relevante, comentaremos a absolutividade para as fórmulas não triviais, e sempre explicitaremos as fórmulas não absolutas.

Teorema 1.3.8. Sejam M uma classe transitiva modelo de $\mathrm{ZF}$ e $\varphi$ uma $\Delta_{1}^{\mathrm{ZF}}$ fórmula. Então $\varphi$ é absoluta para M.

Como foi dito anteriormente, a relação de satisfação "=" para estruturas que são conjuntos é definível. Mais do que isso, essa relação é $\Delta_{1}^{\mathrm{ZF}}$ e, portanto, absoluta para modelos transitivos de ZF. Para o caso das classes próprias, isso não é possível pelo Teorema da Indefinibilidade de Tarski, mas é relevante que para cada $n$, é possível definir a relação de satisfação para cada classe $\Sigma_{n}$ da hierarquia de Levy. Resumimos os comentários desse parágrafo a seguir.

Teorema 1.3.9. Se $M$ é um conjunto então a relação $\{(M,\ulcorner\varphi\urcorner) \mid \varphi \in$ Form $\}$ é $\Delta_{1}^{\mathrm{ZF}}$. Essa relação é $=$ em $M$.

Definição 1.3.10. Sejam M uma classe transitiva e $n<\omega$. A relação $\models_{n}^{\mathrm{M}}$ é definida recursivamente da seguinte forma:

- $\models_{0}^{\mathrm{M}} \varphi\left(x_{1}, \ldots, x_{n}\right) s e$ : $\varphi \in \Sigma_{0} \wedge \exists y \in \mathrm{M}\left(y\right.$ é transitivo $\left.\wedge x_{1}, \ldots, x_{n} \in y \wedge(y, \in) \models \varphi\left(x_{1}, \ldots, x_{n}\right)\right)$. 
- $=_{n+1}^{\mathrm{M}} \exists x \varphi\left(x_{1}, \ldots, x_{n}\right)$ se:

$$
\varphi \in \Pi_{n} \wedge \exists x \in \mathrm{M} \neg \models_{n}^{\mathrm{M}} \neg \varphi\left(x, x_{1}, \ldots, x_{n}\right) .
$$

Agora vamos considerar alguns modelos clássicos para teorias de conjuntos. Alguns desses modelos são classes próprias, e embora a relação de satisfação não seja definível para classes próprias, estamos assumindo o sentido de relativização. Vamos começar com a hierarquia cumulativa de von Neumann, que já apareceu nos trabalhos de von Neumann para motivar a introdução do Axioma da Fundação.

Definição 1.3.11. A hierarquia cumulativa de von Neumann - ou, simplesmente, hierarquia cumulativa - é definida por recursão transfinita da seguinte forma:

- $V_{0}=\emptyset$;

- $V_{\alpha+1}=\mathcal{P}\left(V_{\alpha}\right)$;

- $V_{\gamma}=\bigcup_{\alpha<\gamma} V_{\alpha}$, se $\gamma$ é ordinal limite;

- $\mathrm{WF} \doteq \bigcup_{\alpha \in \mathrm{ON}} V_{\alpha}$.

Cada $V_{\alpha}$ trivialmente é $\in$-transitivo. O Axioma da Fundação é equivalente à escrever a fórmula "V = WF". Essa hierarquia tem papel estrutural fundamental. A grosso modo, ela diz que todo conjunto é construído a partir do vazio, iterando a operação $\mathcal{P}$, e tomando uniões. Diversas construções recursivas são amparadas nessa hierarquia, simplesmente porque cada conjunto pode ser associado a um ordinal, que chamaremos de posto.

Definição 1.3.12. Seja $x$ um conjunto qualquer, o posto de $x$ é definido por

$$
\rho(x)=\min \left\{\alpha \in \mathrm{ON} \mid x \in V_{\alpha+1}\right\}
$$

A noção de "posto" aparecerá mais algumas vezes, sob diversas formas, e deve ser entendida de acordo com o contexto. Cada $V_{\alpha}$ é simplesmente o conjunto dos elementos de posto menor do que $\alpha$. Um resultado clássico é que quando $\gamma>\omega$ é um ordinal limite, $V_{\gamma}$ é modelo de ZC. Para o caso em que $\kappa$ é um cardinal inacessível, temos ainda que $\mathrm{V}_{\kappa}=\mathrm{ZFC}$.

Um exemplo de modelo para ZFC-P é o modelo dos conjuntos de cardinalidade hereditariamente menor do que $\kappa$, onde $\kappa$ é um cardinal regular não-enumerável.

Definição 1.3.13. Seja $\kappa$ um cardinal. Definimos os conjuntos de cardinalidade hereditariamente menor do que $\kappa$ por

$$
H_{\kappa} \doteq\{x \in \mathrm{WF}|| \operatorname{trcl}(x) \mid<\kappa\} .
$$

Como já foi dito, se $\kappa$ é um cardinal regular não-enumerável, $H_{\kappa}=\mathrm{ZFC}-\mathrm{P}$. Resumindo os comentários acima:

\section{Teorema 1.3.14.}

1. $V_{\gamma}=\mathrm{ZC}$, se $\gamma$ é um ordinal limite não-enumerável.

2. $V_{\kappa}=\mathrm{ZFC}$ se $\kappa$ é inacessivel. 
3. $H_{\kappa}=\mathrm{ZFC}-\mathrm{P}$, se $\kappa$ é cardinal regular não-enumerável.

Com a importante noção de definibilidade sobre estruturas, introduzimos também duas construções amparadas na hierarquia cumulativa que são importantes.

Definição 1.3.15. Sejam $\mathcal{L}$ uma linguagem e $\mathcal{M}$ uma $\mathcal{L}$-estrutura. Dizemos que um conjunto $Y$ é definivel sobre $\mathcal{M}$ se existem uma $\mathcal{L}$-fórmula $\varphi\left(x_{0}, x_{1}, \ldots, x_{n}\right)$ e $a_{1}, \ldots, a_{n}$ no domínio de $\mathcal{M}$ tais que

$$
y \in Y \leftrightarrow \mathcal{M} \models \varphi\left(y, a_{0}, \ldots, a_{n}\right)
$$

\section{Definição 1.3.16.}

- Para cada $\alpha \in \mathrm{ON}$, definimos $O D_{\alpha}^{\omega} \doteq\left\{x \in V_{\alpha} \mid x\right.$ é definivel sobre $\left.\left(V_{\alpha} \cap \mathrm{ON}^{\omega}, \in\right)\right\}$;

- A classe dos conjuntos sequencialmente ordinal definiveis é definida por

$$
\mathrm{OD}^{\omega}=\bigcup_{\alpha \in \mathrm{ON}} O D_{\alpha}^{\omega}
$$

Definição 1.3.17. A classe dos conjuntos hereditariamente sequencialmente ordinal definiveis é definida por $\mathrm{HOD}^{\omega} \doteq\left\{x \in \mathrm{OD}^{\omega} \mid \operatorname{trcl}(x) \subseteq \mathrm{OD}^{\omega}\right\}$.

Essas duas últimas definições servem para a Seção 5.2, onde HOD ${ }^{\omega}$ será construído dentro de um modelo de ZFC, fornecendo um modelo que satisfaz ZF + DC, onde DC é o Princípio da Escolha Dependente.

Encerramos esta seção fazendo uma breve discussão sobre relações bem-fundadas e como "transformar" uma relação bem-fundada na relação de pertinência. Isso será feito usando o colapso transitivo de Mostowski.

Definição 1.3.18. Sejam $M$ um conjunto qualquer e $E \subseteq M^{2}$ uma relação irreflexiva. Dizemos que $E$ é bem-fundada em $M$ se para todo subconjunto não vazio $X \subseteq M$, existe um elemento E-minimal.

Teorema 1.3.19. Sejam $M$ um conjunto e $E \subseteq M^{2}$ uma relação irreflexiva. Então $E$ é uma relação bem-fundada em $M$ se, e somente se, existe um homomorfismo $\rho:(M, E) \rightarrow(\mathrm{ON}, \in)$. Além disso, a fórmula " $E$ é bem-fundada" é $\Delta_{1}^{\mathrm{ZF}}$, e portanto, absoluta para modelos $\in$-transitivos de $\mathrm{ZF}$.

Esse homomorfismo é simplesmente um mapa que preserva a "ordem". Ele é dado explicitamente na próxima definição.

Definição 1.3.20. Sejam $M$ um conjunto e E uma relação bem-fundada em $M$, definimos a função posto $\rho: M \rightarrow$ ON recursivamente por

$$
\rho(x)=\{\rho(y)+1 \mid x E y\} .
$$

Outra situação importante é quando uma relação bem-fundada se comporta de forma parecida com a relação de pertinência, o que quer dizer que a estrutura $(M, E)$ satisfaz o Axioma da Extensionalidade. Veremos que nesse caso essa relação "é" essencialmente a relação de pertinência. 
Definição 1.3.21. Sejam $M$ um conjunto e $E \subseteq M^{2}$ uma relação em $M$. Dizemos que $E$ é extensional em $M$ se

$$
\forall x, y \in M((x E y \leftrightarrow y E x) \rightarrow x=y)
$$

Teorema 1.3.22. Sejam $(M, E)$ uma estrutura com $E$ uma relação bem-fundada $M$. Então existe um único homomorfismo sobrejetor $\pi_{M, E}:(M, E) \rightarrow(\bar{M}, \in)$, definido recursivamente por

$$
\pi_{M, E}(x)=\left\{\pi_{M, E}(y) \mid y E x\right\} .
$$

Se, além disso, E é uma relação extensional em $M$, esse homomorfismo é um isomorfismo.

Definição 1.3.23. Sejam $(M, E)$ uma estrutura com E uma relação bem-fundada M. O homomorfismo $\pi_{M, E}$ do Teorema 1.3.22 é dito colapso transitivo de Mostowski - ou, simplesmente, colapso transitivo - da estrutura $(M, E)$.

\subsection{O estudo da regularidade ao longo de hierarquias de complexi- dade}

A teoria descritiva dos conjuntos tem origem como sendo a teoria definivel do continuum - i.e., o estudo de propriedades estruturais para conjuntos definíveis, em um sentido específico, de reais. Essas propriedades são propriedades que indicam bom comportamento, ou regularidade.

Foram os matemáticos franceses Emile Borel, René Baire e Henri Lebesgue que iniciaram o estudo dos subconjuntos definíveis de reais. Eles não só retomaram a abordagem de Cantor para resolver o Problema do Continuum via propriedade do conjunto perfeito, como levaram o estudo dos conjuntos definíveis de números reais a um novo nível de complexidade.

Borel em sua tese de 1898 [Bor98] introduziu para a sua teoria da medida a família dos conjuntos que consistem por intervalos abertos, fechando por uniões enumeráveis e complementação. Essa família é conhecida hoje como a família dos borelianos da reta.

Definição 1.4.1. $\mathcal{B}\left(\left(\omega^{\omega}\right)^{k}\right)$ é a $\sigma$-álgebra gerada pelos abertos básicos de $\left(\omega^{\omega}\right)^{k}$, a $\sigma$-álgebra dos conjuntos borelianos.

É possível construir uma teoria da medida sobre $\mathcal{B}\left(\omega^{\omega}\right)$. Considere $\mu_{\omega}$ a medida em $\mathcal{P}(\omega)$ definida por

$$
\mu_{\omega}(\{n\})=\frac{1}{2^{n+1}}, \text { se } n<\omega \text {, e então } \mu_{\omega}(a)=\sum_{n \in a} \frac{1}{2^{n+1}} \text {, se } a \subseteq \omega .
$$

A partir disso, é possível definir uma medida $\mu_{\mathcal{B}}$ em $\mathcal{B}\left(\omega^{\omega}\right)$, como sendo a medida produto em $\mathcal{B}\left(\omega^{\omega}\right)$ induzida por $\mu_{\omega}$. Explicitamente, se $s \in \omega^{<\omega} \backslash\{\emptyset\}$,

$$
\mu_{\mathcal{B}}\left(\mathcal{O}_{s}\right) \doteq \prod_{n<|s|} \frac{1}{2^{s(n)+1}}
$$

e $\mu_{\mathcal{B}}$ é a única medida em $\mathcal{B}\left(\omega^{\omega}\right)$ com essa propriedade.

Em 1899 [Bai99], Baire em sua tese construiu uma teoria de funções reais que fez uso pela primeira vez depois de Cantor de uma hierarquia transfinita: as funções de Baire classe $\alpha$, onde $\alpha$ é um ordinal $<\omega_{1}$. Baire também desenvolveu em sua tese os conceitos de categoria e Baire mensurabilidade. Os conjuntos de reais Baire mensuráveis são aqueles que só não são abertos por uma "medida negligenciável". Logo veremos que "negligenciável" quer dizer "pertence a um $\sigma$-ideal".

\section{Definição 1.4.2.}

1. Dizemos que um conjunto $F \subseteq \omega^{\omega}$ é raro, se $\operatorname{int}(\bar{A})=\emptyset$. Ou, equivalentemente, $A \cap O=\emptyset$, para todo aberto denso $O$ (em inglês, nowhere dense set). 
2. Dizemos que um conjunto $A \subseteq \omega^{\omega}$ é magro, se existe uma família $\left(F_{n}\right)_{n<\omega}$ de conjuntos raros tal que

$$
A=\bigcup_{n<\omega} F_{n}
$$

3. Dizemos que um conjunto $A \subseteq \omega^{\omega}$ é Baire mensurável se existe um aberto $O$ tal que $A \Delta O$ é magro.

Primeiramente, note que a união enumerável de conjuntos magros é um conjunto magro. Isso quer dizer que a coleção dos conjuntos magros é o $\sigma$-ideal gerado pelos conjuntos raros, e denotaremos esse ideal por $\mathcal{M}$. Além disso, cada conjunto magro está contido em um boreliano (nesse caso, inclusive, é boreliano), e nesse caso diremos que $\mathcal{M}$ é Borel gerado. Os $\sigma$-ideais Borel gerados que aparecerem serão destacados, pois desempenharão um papel importante nesse texto.

Definição 1.4.3. Definimos $\mathcal{M}$ como sendo o $\sigma$-ideal Borel gerado dos conjuntos magros.

Um argumento canônico de indução boreliana (i.e., provar que uma certa propriedade vale para os abertos e é fechada por união enumerável e complementação) implica que todos os borelianos são Baire mensuráveis.

Influenciado pelos trabalhos de Borel e Baire, Lebesgue em sua tese (1902 [Leb02]) desenvolveu sua teoria de medida e integração. Sua teoria de medida generalizava a teoria de Borel, enquanto sua teoria de funções mensuráveis generalizava a teoria das funções de Baire classe $\alpha$. Em seu célebre trabalho de 1905 [Leb05], Lebesgue correlacionou as funções de Baire classe $\alpha$ com os borelianos, mostrando que os borelianos são exatamente as pré-imagens $f^{-1}(O)$ de abertos $O$ por funções $f$ de Baire classe $\alpha$, relacionando a hierarquia de Baire com uma hierarquia natural para os borelianos. A hierarquia canônica de Borel que conhecemos é a apresentada por Hausdorff em 1914 [Hau14], que difere pouco da hierarquia introduzida por Lebesgue.

Definição 1.4.4. Sejam $0<k<\omega$ e $0<\alpha<\omega_{1}$. As classes $\boldsymbol{\Sigma}_{\alpha}^{0}, \boldsymbol{\Pi}_{\alpha}^{0}$ e $\boldsymbol{\Delta}_{\alpha}^{0}$ de subconjuntos de $\left(\omega^{\omega}\right)^{k}$ são definidas recursivamente da seguinte forma. Seja $A \subseteq\left(\omega^{\omega}\right)^{k}$.

- $A \in \Sigma_{1}^{0}$ se, e somente se, $A$ é aberto em $\left(\omega^{\omega}\right)^{k}$.

- $A \in \Pi_{1}^{0}$ se, e somente se, $A$ é fechado em $\left(\omega^{\omega}\right)^{k}$.

- $A \in \boldsymbol{\Sigma}_{\alpha+1}^{0}$ se, e somente se, $A=\bigcup_{n<\omega} B_{n}$ e cada $B_{n} \in \mathbf{\Pi}_{\alpha}^{0}$.

- $A \in \mathbf{\Pi}_{\alpha}^{0}$ se, e somente se, $\left(\omega^{\omega}\right)^{k} \backslash A \in \boldsymbol{\Sigma}_{\alpha}^{0}$.

- $\boldsymbol{\Sigma}_{\gamma}^{0}=\bigcup_{\delta<\gamma} \boldsymbol{\Sigma}_{\delta}^{0}$, se $\gamma$ é ordinal limite.

- $\Delta_{\alpha}^{0}=\boldsymbol{\Sigma}_{\alpha}^{0} \cap \Pi_{\alpha}^{0}$.

O seguinte diagrama representa a hierarquia boreliana .

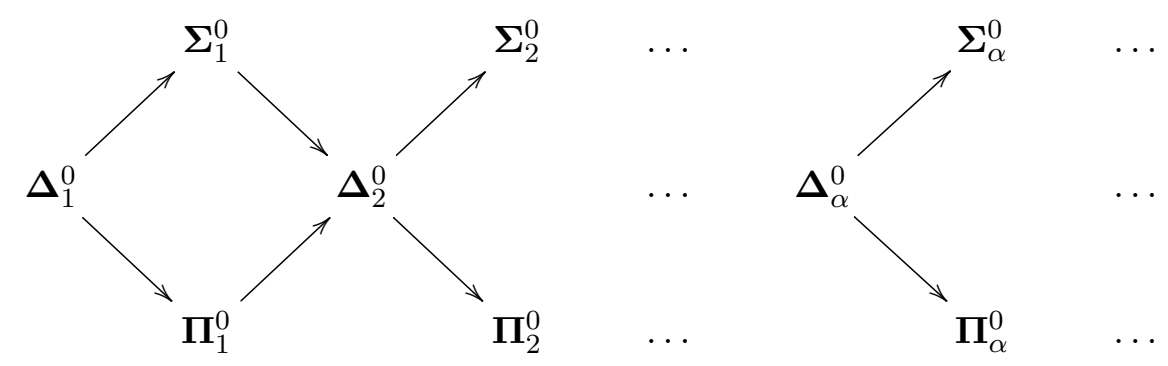

Teorema 1.4.5. Se $k \geq 1$, então

$$
\mathcal{B}\left(\left(\omega^{\omega}\right)^{k}\right)=\bigcup_{0<\alpha<\omega_{1}} \Sigma_{\alpha}^{0}=\bigcup_{0<\alpha<\omega_{1}} \Pi_{\alpha}^{0} .
$$


A direção da flecha representa $\subsetneq$. Portanto, está implícito que essa hierarquia é própria. Lebesgue em seu [Leb05] mostrou que essa hierarquia é de fato própria, provando que a hierarquia das funções de Baire classe $\alpha$ é própria. Pela primeira vez, ficou clara a necessidade de se considerar ordinais além de $\omega$, somando para a inevitável aceitação do ordinal $\omega_{1}$. A seguinte definição revela de forma simples como a medida de Lebesgue generaliza a medida de Borel, e ao mesmo tempo se assemelha à Definição 1.4.2, de Baire mensurabilidade.

\section{Definição 1.4.6.}

1. Um conjunto $N \subseteq \omega^{\omega}$ é dito nulo se existe um boreliano $B$ tal que $\mu_{\mathcal{B}}(B)=0$ e $N \subseteq B$.

2. Um conjunto $A \subseteq \omega^{\omega}$ é dito Lebesgue mensurável se existe um boreliano $B$ tal que $A \Delta B$ é nulo. Nesse caso, definimos

$$
\mu_{L}(A) \doteq \mu_{\mathcal{B}}(B)
$$

Assim como no caso dos conjuntos magros, o ideal $\mathcal{N}$ dos conjuntos de medida nula é um $\sigma$-ideal Borel gerado.

Definição 1.4.7. Definimos $\mathcal{N}$ como sendo o $\sigma$-ideal Borel gerado dos conjuntos nulos.

Trivialmente todos os borelianos são Lebesgue mensuráveis. Por um lado, as noções de Lebesgue mensurabilidade e Baire mensurabilidade são bastante análogas, por outro elas são bem diferentes ${ }^{1}$. Com efeito:

Teorema 1.4.8. Existe um conjunto nulo $N \subseteq \omega^{\omega}$ tal que $\omega^{\omega} \backslash N$ é magro.

Ainda em [Leb05], Lebesgue construiu explicitamente um conjunto Lebesgue mensurável que não é boreliano, mostrando que a hierarquia de Borel não esgota todos os conjuntos de reais.

Durante os próximos anos a medida de Lebesgue se tornaria amplamente aceita como uma medida de regularidade, em conjunto com a Baire mensurabilidade e a propriedade do conjunto perfeito. Não era claro, porém, a extensão dessas propriedades para os conjuntos de reais. Assim, em 1905 [Vit05] Vitali construiu, fazendo uso do Axioma da Escolha, um conjunto de reais não Lebesgue mensurável, que também serviu de exemplo de conjunto não Baire mensurável. Em 1907 [Ber07] Bernstein construiu, também fazendo uso do Axioma da Escolha, um conjunto que não possui a propriedade do conjunto perfeito, um conjunto de Bernstein.

Teorema 1.4.9 (Vitali [Vit05]). Existe um conjunto $A \subseteq \omega^{\omega}$ que não é Lebesgue mensurável, nem Baire mensurável.

Essa prova de Vitali, como esperado, não é construtiva. Não estava claro, portanto, quais conjuntos não seriam Lebesgue mensuráveis. Esse problema é conhecido como Problema da Medida:

“Alguém consegue 'nomear' um conjunto não mensurável?" [Leb05].

Teorema 1.4.10 (Bernstein [Ber07]). Existe um conjunto $A \subseteq \omega^{\omega}$ tal que $A \cap P \neq \emptyset$ e $P \backslash A \neq \emptyset$, para todo conjunto perfeito $P \subseteq \omega^{\omega}$.

Definição 1.4.11. Um conjunto como acima é dito conjunto de Bernstein .

Ao contrário das propriedades de Baire e Lebesgue, que trivialmente valem para os conjuntos borelianos, não era clara a extensão da propriedade do conjunto perfeito para a hierarquia de Borel, já que uma simples indução boreliana não parece possível: note que um conjunto ter a propriedade do conjunto perfeito não implica que seu complementar também tem. Veremos no Capítulo 4 que a

\footnotetext{
${ }^{1}$ Veja o texto de Oxtoby [Oxt13] para uma excelente exposição das analogias e diferenças entre esses dois conceitos, assumindo somente ZFC
} 
propriedade do conjunto perfeito se enquadra em uma propriedade de regularidade do tipo dicotomia (Definição 5.1.10), enquanto as propriedades de Baire e Lebesgue se enquadram nas propriedades de regularidade do tipo mensurabilidade (Definição 5.1.14), e esses dois tipos de propriedades são potencialmente bem diferentes.

Familiarizado com o trabalho de Borel, Baire e Lebesgue enquanto estudante em Paris, Luzin deu continuidade ao estudo dos conjuntos descritivos, fundando o seminário sobre "teoria descritiva das funções" em 1914 na Universidade de Moscow. Foi Aleksandrov [Ale16], aluno de Luzin, quem estabeleceu pela primeira vez a propriedade do conjunto perfeito para os conjuntos borelianos, assim provando que a hierarquia dos borelianos satisfaz CH. Na Seção 2.4, no Teorema 2.4.11, é apresentada a prova de Davis desse fato, via determinância boreliana para jogos infinitos.

Teorema 1.4.12. Se $A \subseteq \omega^{\omega}$ é boreliano, então A tem a propriedade do conjunto perfeito. Consequentemente, vale a Hipótese do Continuum para os conjuntos borelianos.

Demonstração. Veja o Teorema 2.4.11.

Enquanto estudava [Leb05] de Lebesgue, outro estudante de Luzin, Souslin, fez uma descoberta em 1916. Lebesgue cometeu o erro de afirmar que a projeção de um boreliano do plano é um conjunto boreliano. Percebendo isso, Suslin deu um contraexemplo para essa afirmação, dando origem, em 1917 [Sus17], ao que conhecemos como conjuntos analíticos, o que Suslin chamou de conjuntos $A$. Mais tarde, em 1925, Luzin [ $\left.\mathrm{L}^{+} 25\right]$ e Sierpinski [Sie25] estenderam a noção de conjunto analítico, iterando a operação de projeção e tomando complementos, dando origem à hierarquia projetiva.

Definição 1.4.13. Sejam $0<k, n<\omega$. As classes $\boldsymbol{\Sigma}_{n}^{1}$, $\boldsymbol{\Pi}_{n}^{1}$ e $\boldsymbol{\Delta}_{n}^{1}$ de subconjuntos de $\left(\omega^{\omega}\right)^{k}$ são definidas recursivamente da seguinte forma. Seja $A \subseteq\left(\omega^{\omega}\right)^{k}$.

- $A \in \boldsymbol{\Sigma}_{1}^{1}$ se, e somente se, $A=p B$, para algum $B \subseteq\left(\omega^{\omega}\right)^{k+1}$ boreliano. Dizemos que $A$ é analítico.

- $A \in \Pi_{1}^{1}$ se, e somente se, $\left(\omega^{\omega}\right)^{k} \backslash A$ é analítico. Dizemos que A é coanalítico.

- $A \in \boldsymbol{\Sigma}_{n+1}^{1}$ se, e somente se, $A=p B$, para algum $B \subseteq\left(\omega^{\omega}\right)^{k+1}$ tal que $B \in \mathbf{\Pi}_{n}^{1}$.

- $A \in \boldsymbol{\Pi}_{n}^{1}$ se, e somente se, $\left(\omega^{\omega}\right)^{k} \backslash A \in \boldsymbol{\Sigma}_{n}^{1}$.

- $\Delta_{n}^{1}=\boldsymbol{\Sigma}_{n}^{1} \cap \Pi_{n}^{1}$.

O seguinte diagrama representa a hierarquia projetiva .

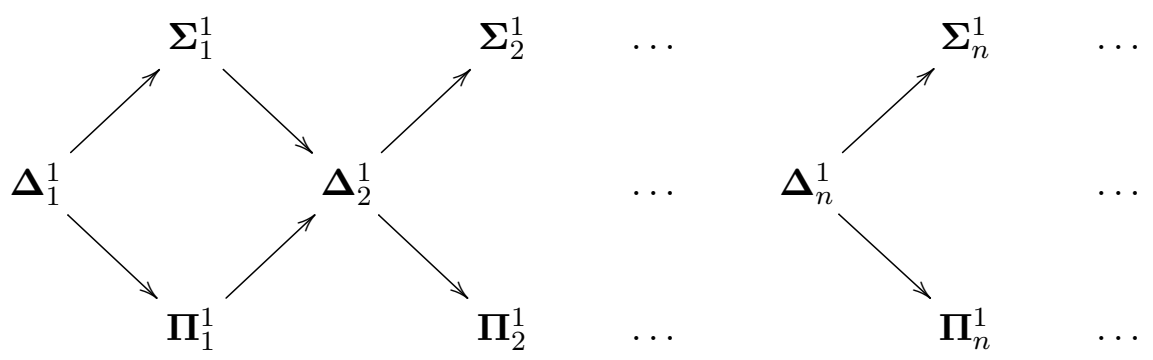

Suslin [Sus17] provou que os borelianos são exatamente os conjuntos analíticos-coanalíticos (i.e., $\Sigma_{1}^{1} \cap \Pi_{1}^{1}$ ), e que existe um conjunto analítico que não é boreliano, sendo o exemplo o mesmo que Lebesgue tinha construído de conjunto Lebesgue mensurável não boreliano. Luzin [Luz17] anunciou que as propriedades de Baire, Lebesgue e a propriedade do conjunto perfeito valem para os conjuntos analíticos. Luzin $\left[\mathrm{L}^{+} 25\right]$ e Sierpinski [Sie25] provaram que essa hierarquia é própria, de forma análoga à como Lebesgue fez usando o método diagonal de Cantor.

Teorema 1.4.14 (Suslin [Sus17]). Seja $0<k<\omega$. 
1. $\boldsymbol{\Delta}_{1}^{1}=\mathcal{B}\left(\left(\omega^{\omega}\right)^{k}\right)$.

2. $\Sigma_{1}^{1} \backslash \Delta_{1}^{1} \neq \emptyset$.

Teorema 1.4.15 (Luzin [Luz17],). Se $A \subseteq \omega^{\omega}$ é um conjunto analítico, (i.e., $A \in \Sigma_{1}^{1}$ ), então

1. A é Lebesgue mensurável.

2. A é Baire mensurável.

3. A tem a propriedade do conjunto perfeito.

As provas de 1. e 2. apareceram em [LS18] e [LS23], 3. só apareceu em [Lus26] e esse resultado é atribuido a Suslin.

Teorema 1.4.16 (Luzin [L+25], Sierpinski [Sie25]). Para todo $0<n<\omega, \boldsymbol{\Sigma}_{n}^{1} \nsubseteq \boldsymbol{\Delta}_{n}^{1}, \boldsymbol{\Pi}_{n}^{1} \nsubseteq_{\boldsymbol{\Delta}_{n}}^{1} e$ $\Delta_{n+1}^{1} \nsubseteq \boldsymbol{\Sigma}_{n}^{1} \cup \boldsymbol{\Pi}_{n}^{1}$.

Os esforços de Luzin na tentativa de estabelecer as três propriedades de regularidade clássicas para os conjuntos projetivos, especialmente a propriedade do conjunto perfeito para os coanalíticos, o levaram a fazer especulações sobre as limitações para resolver esses problemas.

"A teoria dos conjuntos analíticos apresenta uma harmonia perfeita: todo conjunto analítico ou é enumerável ou tem a cardinalidade do continuum; um conjunto analítico nunca é de terceira categoria [é sempre Baire mensurável]... finalmente, um conjunto analítico é sempre [Lebesgue] mensurável.

Resiste, porém, uma considerável lacuna: não se sabe se qualquer conjunto não enumerável complementarmente analítico (isto é, complemento de analítico) tem a cardinalidade do continuum.

Os esforços que depositei na resolução dessa questão me levaram à totalmente inesperada descoberta: existe uma família... consistindo de conjuntos efetivos [definiveis] tal que não se sabe, ou jamais se saberá, se qualquer conjunto dessa família, se não enumerável então possui a cardinalidade do continuum, ou se é de terceira categoria, ou se é mensurável... Essa é a família dos conjuntos projetivos do Sr. H. Lebesgue. Vale, porém, reconhecer a natureza desse novo desenvolvimento."

É claro que Luzin não imaginava algo como "independência de ZFC", já que os matemáticos da época não estavam atentos ao fenômeno da independência. No entanto, em um certo sentido Luzin estava correto, mas a continuidade desse programa - i.e., de estabelecer a regularidade para os conjuntos projetivos - só seria retomada em 1938 com o trabalho de Gödel [Göd38] (veja a Seção $1.5)$.

Apesar do fracasso em estabelecer as propriedades de regularidade para os conjuntos projetivos, outro tipo de propriedade deu frutos: a a propriedade de uniformização. Essa propriedade de uniformização é uma forma de escolha - a saber, uma uniformização para um conjunto $A \subseteq\left(\omega^{\omega}\right)^{2}$ é função $f: \omega^{\omega} \rightarrow \omega^{\omega}$ tal que $f \uparrow p A$ tem gráfico contido em $A$. Luzin introduziu essa propriedade interessado em uniformizações definiveis.

Definição 1.4.17. Sejam $A \subseteq \omega^{\omega}$ e $f: \omega^{\omega} \rightarrow \omega^{\omega}$ uma função. Dizemos que $f$ uniformiza $A$ se

$$
\forall x(\exists y(x, y) \in A \rightarrow(x, f(x)) \in A)
$$

Nosso interesse então é saber quando um conjunto em uma classe projetiva $\boldsymbol{\Gamma}$ pode ser uniformização por uma função cujo gráfico está na classe projetiva $\boldsymbol{\Lambda}$. Por exemplo, Novikov provou que todo conjunto $\boldsymbol{\Pi}_{1}^{1}$ pode ser uniformizado por uma função $\boldsymbol{\Sigma}_{2}^{1}$, e Kondô em 1937 [Kon37] provou o seguinte: 
Teorema 1.4.18 (Teorema da Uniformização de Kondô [Kon37]). Todo subconjunto $\Pi_{1}^{1}$ de $\left(\omega^{\omega}\right)^{2}$ pode ser uniformizado por uma função (cujo gráfico é) $\boldsymbol{\Pi}_{1}^{1}$.

Embora as propriedades de uniformização desempenhem um papel central em teoria descritiva dos conjuntos, usaremos apenas o Teorema de Kondô, e de forma auxiliar.

A teoria da recursão teve início na década de 1930 com matemáticos como Gödel, Turing, Church, Kleene, entre outros. Querendo estender o Teorema da Incompletude de Gödel, Kleene em 1943 [Kle43] desenvolveu o estudo das relações aritméticas — relações obtidas por quantificações sobre números naturais em relações recursivas — e em 1955 [Kle55c] [Kle55a] [Kle55b] as relações analíticas - relações que permitem quantificações sobre funções (elementos de $\omega^{\omega}$ ).

Seja $r \in \omega^{\omega}$. Definimos a linguagem da aritmética parametrizada por $r$ como sendo

$$
\mathcal{L}_{\mathcal{A}(r)} \doteq\left\{\dot{\omega}, \omega^{\dot{\omega}}, a p,+, \cdot, \exp ,<, 0,1, \dot{r}\right\}
$$

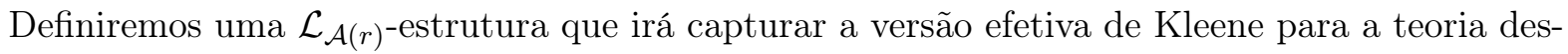
critiva dos conjunto. Na versão efetiva de Kleene, discriminaremos as quantificações sobre números naturais e sobre números reais. Uma das formas de fazer isso é introduzindo quantificadores $\forall^{0}, \exists^{0}$ para variáveis do tipo natural, e quantificadores $\forall^{1} \exists^{1}$ para variáveis do tipo real (ou tipo função), i.e., trabalhando em uma teoria de segunda ordem. Ao invés disso, podemos simular a aritmética de segunda ordem dentro da aritmética de primeira ordem: podemos usar $\dot{\omega}$, um símbolo relacional unário que usaremos para os numeros naturais, $\omega^{\omega}$ símbolo relacional unário para números reais. Com a finalidade de simplificar e seguir textos padrões, vamos convencionar que afirmações do tipo " $\exists n \dot{\omega}(n)$ " e " $\exists x \dot{\omega}^{\omega}(x)$ " são abreviações para $\exists^{0} n$ e $\exists^{1} x$, respectivamente, e analogamente para expressões do tipo " $\forall{ }^{0} n$ " e " $\forall{ }^{1} x$ ". Na linguagem $\mathcal{L}_{\mathcal{A}(r)}$ acima,,$+ \cdot$ e exp são símbolos para as operações usuais nos naturais de soma, produto, e exponenciação, < é símbolo para a relação usual de ordem nos naturais, 0 e 1 são símbolos para as constantes usuais zero e um, ap é a operação de aplicação que conecta os "domínios" e $\dot{r}$ é símbolo para o parâmetro real que introduzimos. Agora, vamos definir a estrutura $\mathcal{A}(r)$.

Definição 1.4.19. Sejam $r \in \omega^{\omega}$ e $\mathcal{L}_{\mathcal{A}(r)}$ a linguagem da aritmética parametrizada por $r$, definimos a $\mathcal{L}_{\mathcal{A}(r) \text {-estrutura canônica como sendo }}$

$$
\mathcal{A}(r) \doteq\left(\omega, \omega^{\omega}, a p,+, \cdot, \exp ,<, 0,1, r\right) .
$$

$\dot{\omega}$ e $\omega^{\omega} \omega$ são interpretados como $\omega$ e $\omega^{\omega}$, respectivamente.,$+ \cdot$, exp $, 0,1,<$ têm as interpretações usuais, como mencionamos anteriormente, $\dot{r}$ é interpretado como sendo $r$. Finalmente o simbolo ap é interpretado como sendo a aplicação ap $: \omega^{\omega} \times \omega \rightarrow \omega$ tal que

$$
a p(x, n)=x(n) .
$$

Além disso, as fórmulas em que todos os quantificadores que aparecem são da forma " $\exists 0 v t$ " ou " $\forall 0 v<s^{0}$ ", para $\mathcal{L}_{\mathcal{A}(r)^{-}}$termos $t$ e $s$ são ditas fórmulas com quantificadores limitados.

Definição 1.4.20. Sejam $r, s, k<\omega$ e uma relação $A \subseteq \omega^{s} \times\left(\omega^{\omega}\right)^{k}$. Dizemos que

1. A é analitica em $r$ se $A$ é definivel em $\mathcal{A}(r)$.

2. A é arimética em $r$ se $A$ é definível em por uma $\mathcal{L}_{\mathcal{A}(r) \text {-fórmula em que não há quantificadores }}$ para funções.

Para introduzir as hierarquias aritmética e analítica de Kleene via alternância de quantificadores, que foram mencionadas anteriormente, precisamos mostrar que sequências de quantificadores limitados sempre podem ser deslocados de forma conveniente, e sequências de quantificadores não limitados " $\exists^{0} \exists^{0}$ " podem ser contraídos em uma única quantificação " $\exists^{0}$ " — isso é análogo a como 
fazemos para contrair duas uniões enumeráveis " $\bigcup_{m<\omega} \bigcup_{n<\omega}$ " em uma única união " $\bigcup_{k<\omega}$ ": usamos bijeções que servem como codificações. A analogia com as heirarquias de Borel e projetiva logo ficará clara.

Definição 1.4.21. Sejam $r, s, k<\omega$ e uma relação $A \subseteq \omega^{s} \times\left(\omega^{\omega}\right)^{k}$. Definimos as relações $\Delta_{0}^{0}(r)$ da seguinte forma: $A \in \Delta_{0}^{0}(r)$ se, e somente se, $A$ é definivel sobre $\mathcal{A}(r)$ por uma $\mathcal{L}_{\mathcal{A}(r) \text {-fórmula }}$ cujos quantificadores são limitados.

Definição 1.4.22. Seja $\left(p_{n}\right)_{n<\omega}$ uma enumeração dos números primos.

1. Para cada $s<\omega$, definimos uma função $\langle\cdot, \ldots, \cdot\rangle: \omega^{s+1} \rightarrow \omega$

$$
\left\langle m_{0}, \ldots, m_{s}\right\rangle=p_{0}^{m_{0}+1} \cdots p_{s}^{m_{s}+1} .
$$

2. Para cada $i<\omega$, definimos $\pi_{n}: \omega \rightarrow \omega$

$$
\pi_{n}(m)=\min \left\{i \mid p_{n}^{i+2} \text { não divide } m\right\} .
$$

A função $\langle\cdot, \ldots, \cdot\rangle$ acima é uma codificação de sequências de naturais de tamanho $s+1$. Trivialmente, todo número natural codifica alguma sequência de tamanho $s$, para algum $s<\omega$. A função $\pi_{n}$ é tal que $\pi_{n}\left(\left\langle m_{0}, \ldots, m_{s}\right\rangle\right)=m_{n}$. Ou seja, $\pi_{n} \circ\langle\cdot, \ldots, \cdot\rangle$ é a projeção na $n$-ésima coordenada. Além disso, note que essas funções são $\Delta_{0}^{0}$. São aritméticas pois $\langle\cdot, \ldots, \cdot\rangle \subseteq \omega^{s+2}$ e $\pi_{n} \subseteq \omega^{2}$, e facilmente podem ser definidas por fórmulas cujos quantificadores são limitados por $s$ e $n$.

Definição 1.4.23. Fixe $s, k<\omega$.

1. Sejam $x \in \omega^{\omega}$ e $m<\omega$. Definimos

$$
\bar{x}(m) \doteq\langle x(0), \ldots, x(m-1)\rangle .
$$

2. Sejam $w \in \omega^{s} \times\left(\omega^{\omega}\right)^{k}, m_{0}, \ldots, m_{s-1} \in \omega$ e $x_{0}, \ldots, x_{k-1} \in \omega^{\omega}$ tais que

$$
w=\left(m_{0}, \ldots, m_{s-1}, x_{0}, \ldots, x_{k-1}\right) .
$$

Para $m<\omega$, definimos $\bar{w}(m) \in \omega^{s+k}$ por

$$
\bar{w}(m) \doteq\left(m_{0}, \ldots, m_{s-1}, \bar{x}_{0}(m), \ldots, \bar{x}_{k-1}\right) .
$$

3. Sejam $x \in \omega^{\omega}$ e $n<\omega$. Definimos $(x)_{n} \in \omega^{\omega}$ da seguinte forma: para cada $m<\omega$, seja

$$
(x)_{n}(m) \doteq x(\langle n, m\rangle) .
$$

Lema 1.4.24. Para relações $\Delta_{0}^{0}, R$ :

1. Podemos mandar todos os quantificadores limitados para a direita:

$$
\left(\forall^{0} p<q\right) \exists^{0} m R(p, q, m, w) \leftrightarrow \exists^{0} m\left(\forall^{0} p<q\right) R\left(p, q, \pi_{p}(m), w\right) .
$$

2. Podemos contrair sequências de quantificadores iguais em um só

$$
\exists^{0} m \exists^{0} n R(m, n, w) \leftrightarrow \exists^{0} p R\left(\pi_{0}(p), \pi_{1}(p), w\right) .
$$


Note que é análogo trocar $\exists^{0}$ por $\forall^{0}$, e a afirmação deve seguir para quantidades finitas arbitrárias de quantificadores trivialmente usando indução. O lema anterior naturalmente sugere que é possível classificar as fórmulas aritméticas numa hierarquia gerada por alternância de quantificadores, como foi feito por Kleene.

Definição 1.4.25. Sejam $0<k, n<\omega, s<\omega$ e $r \in \omega^{\omega}$. As classes $\Sigma_{n}^{0}(r)$, $\Pi_{n}^{0}(r)$ e $\Delta_{n}^{0}(r)$ de relações em $\omega^{s} \times\left(\omega^{\omega}\right)^{k}$ são definidas recursivamente da seguinte forma. Seja $A \subseteq \omega^{s} \times\left(\omega^{\omega}\right)^{k}$.

- $A \in \Sigma_{1}^{0}(r)$ se, e somente se, $\forall w\left(w \in A \leftrightarrow \exists^{0} m R(m, w)\right)$, para alguma relação $R \subseteq \omega^{s+1} \times$ $\left(\omega^{\omega}\right)^{k} \Delta_{0}^{0}(r)$.

- $A \in \Pi_{1}^{0}(r)$ se, e somente se, $\omega^{s} \times\left(\omega^{\omega}\right)^{k} \backslash A \in \Sigma_{1}^{0}(r)$.

- $A \in \Sigma_{n+1}^{0}(r)$ se, e somente se, $\forall w\left(w \in A \leftrightarrow \exists^{0} m R(m, w)\right)$, para alguma relação $R \in \Pi_{n}^{0}(r)$.

- $A \in \Pi_{n}^{0}(r)$ se, e somente se, $\omega^{s} \times\left(\omega^{\omega}\right)^{k} \backslash A \in \Sigma_{n}^{0}(r)$.

- $\Delta_{1}^{0}(r) \doteq \Sigma_{1}^{0}(r) \cap \Pi_{1}^{0}(r)$ é o conjunto das relações recursivas em $r$.

- $\Delta_{n}^{0}(r) \doteq \Sigma_{n}^{0}(r) \cap \Pi_{n}^{0}(r)$.

Essa hierarquia é dita hierarquia aritmética.

Lema 1.4.26. Para relações aritméticas, $R$ :

1. Podemos mandar todos os quantificadores para números naturais para a direita:

$$
\forall^{0} m \exists^{1} x R(m, w, x) \leftrightarrow \exists^{1} x \forall^{0} m R\left(m, w,(x)_{m}\right) .
$$

2. Podemos juntar quantificadores para naturais e reais do mesmo tipo:

$$
\exists^{0} m \exists^{1} x R(m, w, x) \leftrightarrow \exists^{1} x R\left((x)_{0}(0), w,(x)_{1}\right) .
$$

3. Podemos contrair sequências de quantificadores iguais:

$$
\exists^{1} x \exists^{1} y R(w, x, y) \leftrightarrow \exists^{1} z R\left(w,(z)_{0},(z)_{1}\right) .
$$

Definição 1.4.27. Sejam $0<k, n<\omega, s<\omega$ e $r \in \omega^{\omega}$. As classes $\Sigma_{n}^{1}(r)$, $\Pi_{n}^{1}(r)$ e $\Delta_{n}^{1}(r)$ de relações em $\omega^{s} \times\left(\omega^{\omega}\right)^{k}$ são definidas recursivamente da seguinte forma. Seja $A \subseteq \omega^{s} \times\left(\omega^{\omega}\right)^{k}$.

- $A \in \Sigma_{1}^{1}(r)$ se, e somente se, $\forall w\left(w \in A \leftrightarrow \exists^{1} x R(w, x)\right)$, para alguma relação $R \subseteq \omega^{s} \times\left(\omega^{\omega}\right)^{k+1}$ aritmética.

- $A \in \Pi_{1}^{1}(r)$ se, e somente se, $\omega^{s} \times\left(\omega^{\omega}\right)^{k} \backslash A \in \Sigma_{1}^{1}(r)$.

- $A \in \Sigma_{n+1}^{1}(r)$ se, e somente se, $\forall w\left(w \in A \leftrightarrow \exists^{1} x R(w, x)\right)$, para alguma relação $R \in \Pi_{n}^{1}(r)$.

- $A \in \Pi_{n}^{1}(r)$ se, e somente se, $\omega^{s} \times\left(\omega^{\omega}\right)^{k} \backslash A \in \Sigma_{n}^{1}(r)$.

- $\Delta_{n}^{1}(r) \doteq \Sigma_{n}^{1}(r) \cap \Pi_{n}^{1}(r)$.

Essa hierarquia é dita hierarquia analítica .

Lema 1.4.28. Se $n>0$ é par, então existe $\tilde{R} \subseteq \omega^{s+k+n+1}$ uma relação $\Delta_{0}^{0}$ tal que a relação aritmética $R\left(w, x_{1}, \ldots, x_{n}\right)$ como acima pode ser substituida por

$$
\exists^{0} m \tilde{R}\left(m, \bar{w}(m), \bar{x}_{1}(m), \ldots, \bar{x}_{n}(m)\right) .
$$

Analogamente para o caso de $n$ impar trocando $\exists^{0}$ por $\forall^{0}$. 
Agora a analogia entre a hierarquia projetiva e a hierarquia analítica é um teorema:

Teorema 1.4.29. Sejam $A \subseteq\left(\omega^{\omega}\right)^{k}$ e $0<n<\omega$. Então

1. $A \in \boldsymbol{\Sigma}_{n}^{0}$ se, e somente se, existe $r \in \omega^{\omega}$ tal que $A \in \Sigma_{n}^{0}(r)$.

2. $A \in \Sigma_{n}^{1}$ se, e somente se, existe $r \in \omega^{\omega}$ tal que $A \in \Sigma_{n}^{1}(r)$.

Analogamente para as classes $\boldsymbol{\Pi}_{n}^{i}$ e $\boldsymbol{\Delta}_{n}^{i}$, com $i<2$.

\subsection{Em L há contraexemplos para a regularidade dos conjuntos projetivos}

Gödel é provavelmente mais conhecido por seus trabalhos em lógica, relativos à completude e à incompletude da lógica de primeira ordem. Informalmente, a completude estabelece a equivalência entre noções sintáticas e semânticas para a lógica de primeira ordem, e a incompletude impõe limitações sobre o poder de demonstrabilidade para algumas teorias de primeira ordem. Precisamente, para as teorias recursivamente axiomatizáveis e com poder de expressar aritmética, como é o caso da aritmética de Peano, ou de ZF / ZFC. Para o exemplo do caso de ZFC, que é a teoria mais usada para fundamentar toda a matemática, isso significa que, se ZFC for consistente, então existe alguma $\mathcal{L}_{\epsilon}$-sentença que ZFC não pode provar, nem refutar. Mas então, é legítimo perguntar

Quais são essas sentenças?

Como sabemos, um exemplo de sentença do tipo é CH. Assumindo a consistência de ZF, Gödel provou a consistência relativa do Axioma da Escolha e da Hipótese Generalizada do Continuum em 1938 [Göd38]. Gödel fez isso construindo, em ZF, a classe L, o universo dos conjuntos construtíveis.

Teorema 1.5.1. Se ZF é consistente, então também é consistente ZFC com a Hipótese Generalizada do Continuum. Isto é,

$$
\mathrm{Con}(\mathrm{ZF}) \rightarrow \mathrm{Con}(\mathrm{ZFC}+\mathrm{GCH}) .
$$

Quando pensou na classe L, Gödel tinha em mente exatamente o seguinte:

"[Os] conjuntos 'construtíveis' são definidos para serem os conjuntos que podem ser obtidos a partir da hierarquia ramificada de tipos de Russell, se estendida para incluir ordens transfinitas. A extensão para ordens transfinitas tem como consequência que o modelo satisfaz os axiomas impredicativos da teoria dos conjuntos, pois um axioma da redutibilidade pode ser provado para ordens suficientemente grandes." [Göd38].

Hoje em dia, porém, costumamos pensar nesse universo como sendo construído em cada nível na hierarquia cumulativa, de modo que ao invés de tomar as partes no passo sucessor, consideramos somente os subconjuntos definiveis das partes. Com isso, é possível definir indutivamente uma boa ordem para o próximo nível, a partir do anterior. Isso soa como o que o próprio Cantor queria fazer: tentar estabelecer diretamente uma boa ordem para os reais. Essa construção é descrita brevemente a seguir.

Definição 1.5.2. Dado um conjunto $x$, definimos o conjunto dos subconjuntos definiveis de $x$ como sendo

$$
\operatorname{def}(x)=\{y \subseteq x \mid y \text { é definivel sobre }(x, \in)\} .
$$

Definição 1.5.3. A classe dos conjuntos construtiveis é definida por recursão transfinita da seguinte forma: 
- $L_{0}=\emptyset$.

- $L_{\alpha+1}=\operatorname{def}\left(L_{\alpha}\right)$.

- $L_{\gamma}=\bigcup_{\alpha<\gamma} L_{\gamma}$, se $\gamma$ é um ordinal limite.

- $\mathrm{L}=\bigcup_{\alpha \in \mathrm{ON}} L_{\alpha}$.

Além da definição do L, precisaremos também de duas generalizações da construção do L. Assim, fixe um conjunto $A$ qualquer. A primeira construção se refere ao fecho construtivo de $A$, desenvolvida por Hajnal em 1956 [Haj56], e a segunda se refere aos construtíveis com relação a A, desenvolvida por Levy em 1960 [Lév60a].

Definição 1.5.4. Seja A um conjunto. O fecho construtivel de A é definido por recursão transfinita da seguinte forma:

- $L_{0}(A)=\operatorname{trcl}(\{A\})$.

- $L_{\alpha+1}=\operatorname{def}\left(L_{\alpha}(A)\right)$.

- $L_{\gamma}(A)=\bigcup_{\alpha<\gamma} L_{\alpha}(A)$, se $\gamma$ é um ordinal limite.

- $\mathrm{L}(A)=\bigcup_{\alpha \in \mathrm{ON}} L_{\alpha}(A)$.

Primeiramente, note que $\mathrm{L}=\mathrm{L}(\emptyset)$. Agora, $\mathrm{L}(A)$ é sempre um modelo interno de $\mathrm{ZF}$, embora nem sempre seja um modelo de AC. Para que $\mathrm{L}(A)$ seja um modelo de AC é necessário, e suficiente, que $\operatorname{trcl}(A)$ tenha uma boa ordem em $\mathrm{L}(A) . \mathrm{L}(A)$ foi desenvolvido para ser o menor modelo interno que contém $A$ como elemento. A situação para o L $[A]$ é potencialmente diferente:

Definição 1.5.5. Sejam $x, A$ conjuntos. Definimos o conjunto dos subconjuntos definíveis de $x$ a partir de A como sendo

$$
\operatorname{def}^{A}(x)=\{y \subseteq x \mid y \text { é definivel sobre }(x, \in, x \cap A)\},
$$

onde $A \cap x$ é visto como um símbolo relacional unário.

Definição 1.5.6. Seja A um conjunto. Definimos a classe dos conjuntos construtiveis com relação a A por recursão transfinita da seguinte forma:

- $L_{0}[A]=\emptyset$.

- $L_{\alpha+1}=\operatorname{def}^{A}\left(L_{\alpha}[A]\right)$.

- $L_{\gamma}=\bigcup_{\alpha<\gamma} L_{\alpha}$, se $\gamma$ é um ordinal limite.

- $\mathrm{L}[A]=\bigcup_{\alpha \in \mathrm{ON}} L_{\alpha}[A]$.

A primeira diferença do $\mathrm{L}(A)$ para o $\mathrm{L}[A]$ é que $n \tilde{a} o$ temos necessariamente $A \in \mathrm{L}[A]$, embora $A \cap \mathrm{L}[A] \in \mathrm{L}[A]$. A segunda é que $\mathrm{L}[A]$ sempre satisfaz AC. Mas no fim, não faremos diferença entre $\mathrm{L}[A]$ e $\mathrm{L}(A)$, pois, no nosso caso, $A$ sempre será um conjunto de ordinais (inclusive, um número real), o que implica $\mathrm{L}(A)=\mathrm{L}[A]$.

Não iremos muito além disso nas propriedades do L e dessas generalizações. Para uma leitura mais aprofundada referimos textos clássicos como [Kun14], [Jec03] e [Kan08]. Ainda, uma propriedade importante do L vale destacar:

\section{Teorema 1.5.7.}


1. Existe uma $\mathcal{L}_{\in}$-sentença $\sigma$ tal que para qualquer conjunto $M, \in$-modelo para $\mathrm{ZF}$,

$$
M \models \sigma \text { se, e somente se, } M=L_{\delta} \text { para algum ordinal limite } \delta>\omega .
$$

2. Existe uma $\mathcal{L}_{\in}$-fórmula $\varphi\left(v_{0}, v_{1}\right)$ que define em $\mathrm{L}$ uma boa-ordem $<_{L}$ de $L$ tal que para qualquer ordinal limite $\delta>\omega$, para qualquer $y \in L_{\delta}$, e para qualquer $x$,

$$
x<_{L} y \text { se, e somente se, } x \in L_{\delta} \wedge L_{\delta} \mid=\varphi(x, y) .
$$

Além desses resultados famosos de Gödel, pouco se fala sobre outro resultado importante de Gödel, que é o resultado do título dessa seção: em L há contraexemplos para a regularidade dos conjuntos projetivos. Mais precisamente, Gödel anunciou que em L existe um conjunto $\boldsymbol{\Delta}_{2}^{1}$ de reais que não é Lebesgue mensurável, não é Baire mensurável, nem tem a propriedade do conjunto perfeito, embora nunca tenha escrito alguma prova disso. Isso explica (em partes) a dificuldade de Luzin em verificar essas essas propriedades para os conjuntos da hierarquia projetiva. Com isso, Gödel reativou as investigações sobre os conjuntos definíveis de reais: o universo construtível L sendo uma fonte promissora de exploração para noções de regularidade, uniformização, boas ordens, escalas, e diversas outras noções de teoria descritiva dos conjuntos. Nessa época, já se sabia que uma boa ordem para os reais com tipo de ordem $\omega_{1}$ seria suficiente para produzir um conjunto não Lebesgue mensurável (usando um Teorema de Fubini) do plano. A boa ordem $<_{L}$ para todo o universo, quando restrita a $\left(\omega^{\omega}\right)^{2}$, tem tipo de ordem $\omega_{1}$, e Gödel provou que tem complexidade $\Sigma_{2}^{1}$.

Definição 1.5.8. Seja $x \in \omega^{\omega}$.

1. A relação binária codificada por $x$ é definida por

$$
E_{x} \doteq\{(m, n) \mid x(\langle m, n\rangle)=0\} .
$$

2. A $\mathcal{L}_{\in}$-estrutura codificada por $x$ é definida por

$$
M_{x} \doteq\left(\omega, E_{x}\right)
$$

3. Se $E_{x}$ é uma relação bem-fundada e extensional, então denotamos por

$$
\pi_{x}: M_{x} \rightarrow \bar{M}_{x}
$$

o colapso transitivo de $M_{x}$ sobre $\bar{M}_{x}$.

Precisaremos também computar a complexidade para o os reais que codificam relações bemfundadas.

Lema 1.5.9. $\mathrm{WF} \doteq\left\{x \in \omega^{\omega} \mid E_{x}\right.$ é relação bem-fundada $\}$ é $\Pi_{1}^{1}$.

Demonstração. Primeiramente, para $x \in \omega^{\omega}, E_{x}$ ser bem-fundada significa que não existe uma sequência $E_{x}$-decrescente de naturais. Agora, uma sequência de naturais é um número real $y \in \omega^{\omega}$. Assim,

$$
\begin{aligned}
x \in \mathrm{WF} & \leftrightarrow \neg\left(\exists^{1} y \forall^{0} n\left(y(n+1) E_{x} y(n)\right)\right) \\
& \leftrightarrow \forall^{1} y \exists^{0} n(x(\langle y(n+1), y(n)\rangle) \neq \emptyset),
\end{aligned}
$$

o que é trivialmente uma fórmula $\Pi_{1}^{1}$, pois a relação $\langle\cdot, \cdot\rangle$ é recursiva.

Lema 1.5.10. Fixe $\left\{s_{n} \mid n<\omega\right\}$ uma enumeração recursiva de $\omega^{<\omega}$ tal que $\left|s_{n}\right| \leq n$, para todo $n<\omega$. 
1. Para todo $n<\omega$, a relação

$$
\left\{(m, i, x) \in \omega^{2} \times \omega^{\omega} \mid m=\ulcorner\varphi\urcorner \wedge \varphi \in \Sigma_{n} \wedge M_{x} \models \varphi\left(s_{i}\right)\right\}
$$

é aritmética.

2. Para toda coleção $\Gamma$ de $\mathcal{L}_{\epsilon}$-sentenças, o conjunto $\left\{x \in \omega^{\omega}\left|M_{x}\right|=\Gamma\right\}$ é $\Delta_{1}^{1}(\ulcorner\Gamma\urcorner)$.

3. Se $r \in \omega^{\omega}$ e $M_{r}$ é bem-fundado e extensional, então

$$
\left\{(m, x) \in \omega \times \omega^{\omega} \mid \pi_{r}(m)=x\right\}
$$

é uma relação aritmética em $r$.

Teorema 1.5.11. $\left(\omega^{\omega}\right)^{2} \cap<_{L}$ é $\boldsymbol{\Delta}_{2}^{1}$.

Demonstração. Pelo item 2 do Teorema 1.5.7, considere a fórmula $\varphi\left(v_{0}, v_{1}\right)$ e qualquer $\delta>\omega$ ordinal limite tal que $x, y \in L_{\delta} \mathrm{e}$

$$
x<_{L} y \Leftrightarrow L_{\delta}=\varphi(x, y) .
$$

Agora, como o tipo de ordem de $\left(\omega^{\omega},<_{L}\right)$ é $\omega_{1}$, podemos assumir que $\delta<\omega_{1}$. Assim, para $x, y \in \omega^{\omega}$, podemos escrever

$$
x<_{L} y \Leftrightarrow \exists \delta<\omega_{1}\left(x, y \in L_{\delta} \wedge L_{\delta}=\varphi(x, y)\right) .
$$

Por outro lado, todo modelo enumerável pode ser codificado por um número real. Isso significa que o modelo $\left(L_{\delta}, \in\right)$ deve ser isomorfo a um modelo $M_{z}$, para algum real $z$. Nesse caso, passando para o colapso transitivo teremos $L_{\delta}=\bar{M}_{z}$. Além disso, pelo item 1 do Teorema 1.5.7, esse modelo deve satisfaz a sentença $\sigma$, i.e., $\bar{M}_{z} \models \sigma$. Assim,

$$
\begin{aligned}
x<_{\mathrm{L}} y \leftrightarrow \exists^{1} z \exists^{0} m \exists^{0} n\left(M_{z} \text { é extensional e bem-fundado }\right) \\
\left.\wedge \pi_{z}(m)=x \wedge \pi_{z}(n)=y \wedge M_{z}=\sigma \wedge \varphi(m, n)\right) .
\end{aligned}
$$

onde

- " $M_{z}$ é extensional" é trivialmente uma fórmula aritmética em $z$;

- " $M_{z}$ é bem-fundado" é uma fórmula $\Pi_{1}^{1}(z)$, pelo Lema 1.5.9;

- " $\pi_{z}(m)=x "$ (e simetricamente " $\pi_{z}(n)=y$ ") é uma relação aritmética em $z$ pelo ítem 3 do Lema 1.5.10;

- “ $M_{z} \models \sigma \wedge \varphi(m, n)$ " é $\Delta_{1}^{1}(\ulcorner\{\sigma, \varphi(m, n)\}\urcorner)$ pelo ítem 2 do Lema 1.5.10.

Segue que $\left(\omega^{\omega}\right)^{2} \cap<_{L}$ é $\boldsymbol{\Sigma}_{2}^{1}$. Para ver que também é $\boldsymbol{\Pi}_{2}^{1}$, é só usar que para $x, y \in \omega^{\omega}$,

$$
x<_{L} y \leftrightarrow x \neq y \wedge \neg\left(y<_{L} x\right) .
$$

Corolário 1.5.12. Se $\mathrm{V}=\mathrm{L}$, então $\left(\omega^{\omega}\right)^{2} \cap<_{L}$ é uma conjunto $\Delta_{2}^{1}$ que não é Lebesgue mensurável.

Embora isso não seja uma prova para subconjuntos dos reais, o seguinte teorema nos diz que em L existe um conjunto de Bernstein (que é um contraexemplo para a propriedade do conjunto perfeito), e com os resultados do Capítulo 5, veremos que um conjunto de Bernstein não é Lebesgue mensurável, nem Baire mensurável.

Teorema 1.5.13. Se $\mathrm{V}=\mathrm{L}$, então existe um conjunto $\boldsymbol{\Delta}_{2}^{1}$ de Bernstein. 
Demonstração. Seja $\left\{P_{\alpha} \mid \alpha<\aleph_{1}\right\}$ uma $\aleph_{1}$-enumeração de todos os conjuntos perfeitos em L (recorde que $\mathrm{L} \models \mathrm{CH}$ e há c conjuntos perfeitos). Construiremos dois conjuntos, $A$ e $B$, recursivamente em $\alpha<\aleph_{1}$ da seguinte forma: se $A_{\alpha} \doteq\left\{a_{\beta} \mid \beta<\alpha\right\}$ e $B_{\alpha} \doteq\left\{b_{\beta} \mid \beta<\alpha\right\}$ já foram construidos, então selecionamos $a_{\alpha} \doteq \min \left(P_{\alpha} \backslash\left(A_{\alpha} \cup B_{\alpha}\right)\right)$ e $b_{\alpha} \doteq \min \left(P_{\alpha} \backslash\left(A_{\alpha} \cup B_{\alpha} \cup\left\{a_{\alpha}\right\}\right)\right)$ (min, nesse caso, se refere a $<_{\mathrm{L}}$-min). Sejam $A \doteq\left\{a_{\alpha} \mid \alpha<\aleph_{1}\right\}$ e $B=\left\{b_{\alpha} \mid \alpha<\aleph_{1}\right\}$. Temos que $A \cap B=\emptyset \mathrm{e}$ por construção, $A$ e $B$ são conjuntos de Bernstein. É suficiente então mostrar que cada um dos conjuntos $A$ e $B$ é $\boldsymbol{\Sigma}_{2}^{1}$ e, portanto, $\boldsymbol{\Delta}_{2}^{1}$. Faremos para o caso $A$, e para o caso $B$ é igual.

Primeiro, note que se $a_{\alpha} \in L_{\delta}$ para algum ordinal limite enumerável suficientemente grande, então os segmentos iniciais $\left\{a_{\beta} \mid \beta<\alpha\right\}$ e $\left\{b_{\beta} \mid \beta<\alpha\right\}$ também estão em $L_{\delta}$. Além disso, tomar o $<_{L}$-menor elemento pode ser expresso dentro de $L_{\delta}$ usando uma fórmula absoluta que define o segmento inicial da boa ordem $<_{\delta}$. Assim, podemos escrever

$$
x \in A \Leftrightarrow \exists \delta<\omega_{1}\left(L_{\delta} \models x \in A\right) .
$$

De forma análoga à demonstração do teorema anterior, isso pode ser escrito como

$$
\exists^{1} z \exists^{0} m \exists^{0} n\left(M_{z} \text { é extensional e bem-fundado }\right) \wedge \pi_{z}(m)=x \wedge M_{z}=\sigma \wedge \pi_{z}(n) \in A,
$$

o que é uma fórmula $\Sigma_{2}^{1}$. 


\section{Capítulo 2}

\section{Teoria descritiva dos conjuntos}

\subsection{Como codificar borelianos usando números reais}

Veremos na Seção 4.2 que Solovay foi a primeira pessoa a considerar forcings que não são funções parciais (em [Sol70]), definindo um forcing cujas condição são (essencialmente) borelianos de medida positiva (Definição 4.2.4). Assim, dado um boreliano de $\omega^{\omega}$, precisaremos considerar versões relativizadas desse boreliano para diversos modelos, como $\mathrm{V}, \mathrm{V}[G], \mathrm{L}[r]$ etc. Mas temos um problema: a definição de um determinado boreliano não é absoluta para modelos transitivos de ZFC. Para resolver esse problema, Solovay observou que o procedimento para se obter qualquer boreliano pode ser armazenado em um número real. Em outras palavras, todo boreliano pode ser codificado em um número real, de forma análoga a como os abertos $\mathcal{O}_{s}$ são codificados por sequências $s \in \omega^{<\omega}$. O primeiro passo é obter um procedimento para codificar um boreliano, e o segundo passo é obter um procedimento para decodificar um código para um boreliano (i.e., determinar o boreliano correspondente ao código). Para um determinado boreliano armazenado em um código, a decodificação dentro de um modelo transitivo (que contém esse código) é como esse modelo enxerga a definição desse boreliano.

Definição 2.1.1. Sejam $c \in \omega^{\omega}, i<\omega$ e $\Gamma$ a bijeção canônica entre $\omega \times \omega$ e $\omega$. Definimos as sequências auxiliares $u(c), v_{i}(c) \in \omega^{\omega}$ da seguinte forma:

- $u(c)(n)=c(n+1)$

- $v_{i}(c)(n)=c(\Gamma(i, n)+1)$

Agora, se $0<\alpha<\omega_{1}$, definimos as hierarquias $\Sigma_{\alpha}$ e $\Pi_{\alpha}$, recursivamente da seguinte forma:

- $c \in \Sigma_{1}$ se $c(0)>1$

- $c \in \Pi_{\alpha}$ se $c \in \Sigma_{\beta} \cap \Pi_{\beta}$ para algum $\beta<\alpha$ ou $c(0)=0$ e $u(c) \in \Sigma_{\alpha}$

- $c \in \Sigma_{\alpha}$ se $c \in \Sigma_{\beta} \cap \Pi_{\beta}$ para algum $\beta<\alpha$ ou $c(0)=1$ e $v_{i}(c) \in \bigcup_{\beta<\alpha}\left(\Sigma_{\beta} \cup \Pi_{\beta}\right)$ para todo $i<\omega$.

Dizemos que os elementos de $\Sigma_{\alpha}$ são $\Sigma_{\alpha}$-código, e os elementos de $\Pi_{\alpha}$ são $\Pi_{\alpha}$-códigos. O conjunto dos códigos de Borel é definido por

$$
\mathrm{BC}=\bigcup_{\alpha<\omega_{1}} \Sigma_{\alpha}=\bigcup_{\alpha<\omega_{1}} \Pi_{\alpha}
$$

Definição 2.1.2. Sejam $c \in \mathrm{BC} e\left(s_{n} \mid n<\omega\right)$ enumeração recursiva de $\omega^{<\omega}$ tal que $\left|s_{n}\right| \leq n$, para todo $n<\omega$. A decodificação de Borel é a função $c \mapsto B_{c}$ definida da seguinte forma: 


$$
B_{c}= \begin{cases}\bigcup\left\{\mathcal{O}_{s_{n}} \mid c(n)=1\right\} & \text { se } c \in \Sigma_{1} \\ \omega^{\omega} \backslash B_{u(c)} & \text { se } c \in \Pi_{\alpha} \\ \bigcup_{i<\omega} B_{v_{i}(c)} & \text { se } c \in \Sigma_{\alpha} .\end{cases}
$$

Os próximos teoremas nos dizem que esse processo para codificar borelianos e decodificar códigos para borelianos se comporta da forma que esperamos. Além disso, mostraremos que as complexidades de algumas propriedades básicas são limitadas por $\Pi_{1}^{1}$, e na próxima seção veremos que isso é suficiente para assegurar a absolutividade dessas propriedades.

Teorema 2.1.3. Seja $c \in$ BC.

1. Se $c \in \Sigma_{\alpha}$, então $B_{c} \in \Sigma_{\alpha}^{0}$.

2. Se $B \in \Sigma_{\alpha}^{0}\left(\right.$ ou $\left.\Pi_{\alpha}^{0}\right)$, então existe $c \in \Sigma_{\alpha}\left(\right.$ respec. $\left.\Pi_{\alpha}\right)$ tal que $B=B_{c}$.

Demonstração.

1. Segue trivialmente por indução sobre $\alpha<\omega_{1}$.

2. Se $\alpha=1$ e $B \in \boldsymbol{\Sigma}_{\alpha}^{0}$, então $B$ é aberto e

$$
B=\bigcup_{i \in I} \mathcal{O}_{s_{i}}
$$

Para um certo conjunto de índices $i \in I$, onde $\left(s_{n} \mid n<\omega\right)$ é uma enumeração de $\omega^{<\omega}$ tal que $\left|s_{n}\right| \leq n$ para todo $n<\omega$. Assim, bastante tomar $c \in \omega^{\omega}$ tal que $c(0)>1$ e $c(i)=1$ para todo $i \in I$ (podendo estender $c$ arbitrariamente fora de $I$ ). Temos que $c \in \Sigma_{1}$ e $B_{c}=B$.

Suponha que para $\beta<\alpha, B \in \boldsymbol{\Sigma}_{\beta}^{0}$ tenha um código de Borel $c \in \Sigma_{\beta}$ e seja $C=\omega^{\omega} \backslash B \in \boldsymbol{\Pi}_{\beta}^{0}$. Vamos definir $d$ de modo que $d(0)=0$ e $u(d)=c$ :

$$
d(n)= \begin{cases}0 & \text { se } n=0 \\ c(n-1) & \text { se } n>0 .\end{cases}
$$

Assim, $u(d)(n)=d(n+1)=c(n)$, para todo $n<\omega$. Logo, $u(d)=c$. Por definição, $d \in \Pi_{\beta}$. Além disso,

$$
B_{d}=\omega^{\omega} \backslash B_{u(d)}=\omega^{\omega} \backslash B_{c}=\omega^{\omega} \backslash B=B .
$$

Agora, suponha que para todo $\beta<\alpha$, todos os conjuntos $\boldsymbol{\Sigma}_{\beta}^{0}$ têm códigos de Borel. Como consequência do que fizemos anteriormente, também todo $\boldsymbol{\Pi}_{\beta}^{0}$ possui código de Borel. Sejam $\left\{B_{\beta} \mid \beta<\alpha\right\}$ uma família de borelianos em $\boldsymbol{\Pi}_{\beta}^{0}$ e $B \doteq \bigcup_{\beta<\alpha} B_{\beta}$ boreliano em $\boldsymbol{\Sigma}_{\alpha}^{0}$. Considere então para cada $\beta<\alpha$ um $\Pi_{\beta}$-código $c_{\beta}$ que codifica o boreliano $B_{\beta}$.

Podemos reenumerar os códigos $\left\{c_{\beta} \mid \beta<\alpha\right\}$ para $\left\{c_{i} \mid i<\omega\right\}$ pois $|\beta| \leq \aleph_{0}$. Assim, $\left\{c_{i} \mid i<\omega\right\} \subseteq \bigcup_{\beta<\alpha} \Pi_{\beta}$. Para cada $i<\omega$, considere $\beta_{i}$ tal que $c_{i}=\beta_{i}$. Agora vamos definir um $\Sigma_{\alpha}$-código para $B$ :

$$
c(m)= \begin{cases}1 & \text { se } m=0 \\ c_{i}(n) & \text { se } m=\Gamma(n, i)+1\end{cases}
$$

Agora, $c(0)=1$ e $v_{i}(c)(n)=c(\Gamma(n, i)+1)=c_{i}(n)$ e, portanto, $v_{i}(c)=c_{i} \in \Pi_{\beta}$, para todo $i<\omega$. Por definição, $c \in \Sigma_{\alpha}$. 
Além disso,

$$
B_{c}=\bigcup_{i<\omega} B_{v_{i}(c)}=\bigcup_{i<\omega} B_{c_{i}}=\bigcup_{i<\omega} B_{\beta_{i}}=\bigcup_{\beta<\alpha} B_{\beta}=B .
$$

Corolário 2.1.4. Todo boreliano pode ser codificado por um número real. Isto é, a decodificação de Borel é uma função sobrejetora:

$$
\left\{B_{c} \mid c \in \mathrm{BC}\right\}=\mathcal{B}\left(\omega^{\omega}\right) .
$$

Definição 2.1.5. Definimos a relação aritmética $E \subseteq\left(\omega^{\omega}\right)^{2}$ da seguinte forma:

$$
\begin{aligned}
& x E y \leftrightarrow y(0)=0 \wedge x=u(y) \\
& \vee y(0)=1 \wedge \exists^{0} i\left(x=v_{i}(y)\right) .
\end{aligned}
$$

Teorema 2.1.6. $\mathrm{BC} \in \Pi_{1}^{1}$.

Demonstração. Vamos mostrar que

$$
y \in \mathrm{BC} \leftrightarrow \mathrm{E} \text { é uma relação bem-fundada abaixo de } y \text {. }
$$

De fato, é possível mostrar por indução na complexidade do código $y$, usando os seguintes fatos:

- se $y \in \Sigma_{1}$, então $y$ é $E$-minimal;

- se $y \in \Pi_{\alpha}$ e $x E y$, então $x \in \Sigma_{\alpha}$;

- se $y \in \Sigma_{\alpha}$ e $x E y$, então $x \in \bigcup_{\beta<\alpha} \Pi_{\beta}$.

Para a volta, é só usar indução sobre a relação bem-fundada $E$.

Agora,

$E$ é uma relação bem-fundada abaixo de $y \leftrightarrow \nexists\left(z_{n}\right)_{n<\omega}\left(y=z_{0} \wedge \forall n\left(z_{n+1} E z_{n}\right)\right)$,

sendo a expressão da direita uma fórmula $\Pi_{1}^{1}(y)$.

Teorema 2.1.7. Sejam $x, c \in \omega^{\omega}$. Então a propriedade

$$
c \in \mathrm{BC} \wedge x \in B_{c}
$$

$e^{1} \Delta_{1}^{1}$

Demonstração. Podemos definir a relação $R \subseteq\left(\omega^{\omega}\right)^{2} \times \mathcal{P}\left(\omega^{\omega}\right)$ da seguinte forma:

$$
(x, c, T) \in R \leftrightarrow x \in T \wedge c \in T \wedge \forall^{1} z(z E c \rightarrow z \in T) .
$$

Primeiro, note que $T_{(x, c)} \doteq \bigcap\{T \mid(x, c, T) \in R\}$ é enumerável. Assim, podemos supor sem perda de generalidade que $R \subseteq\left(\omega^{\omega}\right)^{2} \times\left[\omega^{\omega}\right]^{\aleph_{0}}$. Além disso, podemos identificar $\left[\omega^{\omega}\right]^{\aleph_{0}}$ com $\omega^{\omega}$ e supor que $R$ é uma relação em $\left(\omega^{\omega}\right)^{3}$.

Também, usando apenas a definição da relação $E$ (Definição 2.1.5), a fórmula $\forall^{1} z(z E c \rightarrow T$ ) pode ser escrita como

$$
(c(0)=0 \rightarrow u(c) \in T) \wedge\left(c(0)=1 \rightarrow \forall^{0} i v_{i}(c) \in T\right) .
$$

Assim, $R$ pode ser vista como uma relação aritmética. 
Agora, defina a relação $S \subseteq\left(\omega^{\omega}\right)^{2} \times\left[\omega^{\omega}\right]^{\aleph_{0}} \times 2^{\left[\omega^{\omega}\right]^{\aleph_{0}}}$ da seguinte forma:

$$
\begin{aligned}
(x, c, T, h) \in S & \leftrightarrow(x, c, T) \in R \\
& \wedge h: T \rightarrow 2 \\
& \wedge c(0)>1 \rightarrow\left(h(c)=1 \leftrightarrow \exists^{0} n\left(c(n)=1 \wedge x \in \mathcal{O}_{s_{n}}\right)\right) \\
& \wedge c(0)=0 \rightarrow(h(c)=1 \leftrightarrow h(u(c))=0) \\
& \wedge c(0)=1 \rightarrow\left(h(c)=1 \leftrightarrow \exists^{0} i h\left(v_{i}(c)\right)=1\right) .
\end{aligned}
$$

Note que existe um único $\subseteq$-menor $T_{(x, c)}$. Também, quando $c$ é um código de Borel, existe uma única $h_{(x, c)}$ tal que $\left(x, c, T_{(x, c)}, h_{(x, c)}\right) \in S$. Assim, podemos considerar apenas uma quantidade $\mathfrak{c}$ de funções $h \in 2^{\left[\omega^{\omega}\right]^{\aleph_{0}}}$ e, portanto, identificar a relação $S$ com uma relação em $\left(\omega^{\omega}\right)^{4}$. Além disso, como a relação $R$ é aritmética em $x, c$, então a relação $S$ é aritmética em $x, c, T, h$.

Por fim,

$$
\begin{aligned}
x \in \mathrm{BC} \wedge x \in B_{c} & \leftrightarrow \forall^{1} T \forall^{1} h((x, c, T, h) \in S \rightarrow h(c)=1) \text { (fórmula } \Pi_{1}^{1} \text { ) } \\
& \leftrightarrow \exists^{1} T \exists^{1} h\left((x, c, T, h) \in S \wedge h(c)=1 \text { ) (fórmula } \Sigma_{1}^{1}\right) .
\end{aligned}
$$

Teorema 2.1.8. As seguintes propriedades são $\Pi_{1}^{1}$ :

1. $B_{c}=\emptyset$

2. $B_{c} \subseteq B_{d}$

3. $B_{c}=B_{d}$

Demonstração.

1. Observe que

$$
B_{c}=\emptyset \leftrightarrow c \in \mathrm{BC} \wedge \forall^{1} x\left(x \notin B_{c}\right) \leftrightarrow \forall^{1} x\left(c \in B C \wedge x \notin B_{c}\right) .
$$

Precisamos verificar que " $c \in \mathrm{BC} \wedge x \notin B_{c}$ " tem complexidade no máximo $\Pi_{1}^{1}$. Para isso, basta observar que essa fórmula é equivalente a " $x \in B C \wedge\left(c \notin B C \vee x \notin B_{c}\right)$ ". Primeiro, " $c \in B C$ " tem complexidade $\Pi_{1}^{1}$. Segundo, como " $c \in B C \wedge x \in B_{c}$ " tem complexidade $\Delta_{1}^{1}$, então " $c \notin B C \vee x \notin B_{c}$ " também tem complexidade $\Delta_{1}^{1}$. Assim, "c $\in B C \wedge x \notin B_{c}$ " tem complexidade " $\Pi_{1}^{1} \wedge \Delta_{1}^{1}$ ", isto é, $\Pi_{1}^{1}$.

2. De forma análoga a como foi feito no item anterior, note que

$$
B_{c} \subseteq B_{d} \leftrightarrow \forall^{1} x\left(\left((d \in \mathrm{BC}) \wedge\left(c \in \mathrm{BC} \wedge x \in B_{c}\right)\right) \vee\left((c \in \mathrm{BC}) \wedge\left(d \in \mathrm{BC} \wedge x \notin B_{d}\right)\right)\right)
$$

é uma fórmula do tipo " $\forall 1 x\left(\left(\Pi_{1}^{1} \wedge \Delta_{1}^{1}\right) \vee\left(\Pi_{1}^{1} \wedge \Pi_{1}^{1}\right)\right)$ " e, portanto, tem complexidade $\Pi_{1}^{1}$.

3. Segue trivialmente do item anterior usando a definição da igualdade.

\subsection{Representação arbórea dos conjuntos analíticos}

No artigo [LS23] de 1923, Luzin e Sierpiński voltaram a atenção também para os conjuntos coanalíticos, fornecendo uma representações para esses conjuntos em termos de árvores bem-fundadas. Com isso, eles conseguiram obter o seguindo resultado: 
Teorema 2.2.1 ([LS23]). Todo conjunto analítico pode ser escrito como união (ou intersecção) de $\aleph_{1}$ borelianos.

Como mencionado anteriormente, Suslin [Sus17] concebeu a noção de conjunto analítico. Suslin descreveu os conjuntos analíticos através de uma operação chamada Operação A. Suslin descreveu um sistema definido como uma família $\left(X_{s}\right)_{s \in \omega<\omega}$ de conjuntos, e definiu a aplicação $A\left(X_{s}\right)_{s \in \omega<\omega}$ sobre um sistema definido da seguinte forma:

$$
x \in A\left(X_{s}\right)_{s \in \omega<\omega} \leftrightarrow \exists^{1} f \forall^{0} n\left(x \in X_{f\lceil n}\right) .
$$

Esse sistema definido é normalmente dito Esquema de Suslin em textos clássicos de teoria descritiva dos conjuntos. Assim, Suslin definiu os conjuntos analíticos como sendo os conjuntos que são imagem da Operação A, para algum Esquema de Suslin. Agora, sejam $Y \subseteq \omega^{\omega}$ um conjunto coanalítico e $X=A\left(X_{s}\right)_{s \in \omega<\omega}$ tal que $Y=\omega^{\omega} \backslash X$. Temos que

$$
x \in Y \leftrightarrow \forall^{1} f \exists^{0} n\left(x \notin X_{f\lceil n}\right) .
$$

Considere a ordem $\supsetneq$ no conjunto $\omega^{<\omega}$ das sequências finitas (i.e., a ordem de estender propriamente). Para um número real $x \in \omega^{\omega}$, defina

$$
T_{x} \doteq\left\{s \in \omega^{<\omega} \mid \forall t \subseteq s\left(x \in X_{t}\right)\right\} .
$$

Assim,

$$
x \in Y \leftrightarrow \supsetneq \text { é uma relação bem-fundada em } T_{x} .
$$

Portanto, relações bem-fundadas desempenham papel crucial na análise dos conjuntos analíticos e coanalíticos. Em um contexto mais geral, a mesma ideia é usada para analisar os conjuntos $\boldsymbol{\Sigma}_{2}^{1}$, ou $\boldsymbol{\Pi}_{2}^{1}$. Definiremos a noção de árvore para englobar o caso mais geral em que consideramos subconjuntos de $\left(\omega^{\omega}\right)^{k}$. Logo ficará clara a importância dessa generalidade. A prova que esboçamos acima é refeita no Teorema 2.2.11, nesse contexto mais geral.

Definição 2.2.2. Sejam $k<\omega, X$ um conjunto qualquer e $s, t \in \bigcup_{m<\omega}\left(\omega^{m}\right)^{k} \times X^{m}$. Suponha que $s=\left(s_{0}, \ldots, s_{k}\right)$ e $t=\left(t_{0}, \ldots, t_{k}\right)$.

1. A concatenaçãoo $\frown^{*}$ de s et é a concatenação coordenada-a-coordenada: $s^{{ }^{*}} t=\left(s_{0} t_{0}, \ldots, s_{k} t_{k}\right)$.

2. A inclusão $\subseteq^{*}$ é a inclusão coordenada-a-coordenada também: $s \subseteq^{*} t \leftrightarrow s_{i} \subseteq t_{i}$ para todo $i \leq n$.

Definição 2.2.3. Sejam $k<\omega$ e $X$ um conjunto qualquer. Dizemos que $T$ é uma árvore em $\omega^{k} \times X$ se

1. $T \subseteq \bigcup_{m<\omega}\left(\omega^{m}\right)^{k} \times X^{m}$.

2. $\left(t_{0}, \ldots, t_{k}\right) \in T$ implica $\left(t_{0}\left\lceil m, \ldots, t_{k}\lceil m) \in T\right.\right.$, para todo $m<\left|t_{0}\right|$.

Os elementos de $T$ são ditos nós.

Para o caso $k=0$, uma árvore $T$ em $X$ é simplesmente um conjunto $T \subseteq X^{<\omega}$ fechado por segmentos iniciais. Veremos árvores especificamente desse tipo, que chamaremos de árvores reais, na Seção $4.3, \operatorname{com} X=\omega$ ou $X=2$

Definição 2.2.4. Sejam $k<\omega, X$ um conjunto e $T$ uma árvore em $\omega^{k} \times X$. Então

1. O conjunto dos ramos de $T$, que também chamamos de corpo de $T$, é definido por

$$
[T]=\left\{\left(x_{0}, \ldots, x_{k-1}, f\right) \in\left(\omega^{\omega}\right)^{k} \times X^{\omega} \mid \forall m<\omega\left(\left(x_{0}\left\lceil m, \ldots, x_{k-1}\lceil m, f\lceil m) \in T)\right\} .\right.\right.\right.
$$


2. $T$ é uma árvore bem-fundada se $[T]=\emptyset$.

3. Seja $t \in T$, definimos

$$
T / t=\left\{s \mid t^{\wedge^{*}} s \in T\right\},
$$

o conjunto das extensões possiveis a partir do nó t.

Sobre o item 1., vale uma observação para o caso particular em que $T$ é uma árvore em $\omega^{k}$ :

Lema 2.2.5. Se é $T$ uma árvore em $\omega^{k}$, então $[T]$ é um subconjunto fechado de $\left(\omega^{\omega}\right)^{k}$. Reciprocamente, se $C$ é um subconjunto fechado de $\left(\omega^{\omega}\right)^{k}$, então existe uma árvore $T$ em $\omega^{k}$ tal que $C=[T]$.

O Item 2. anterior é equivalente, sob DC, à inexistência de cadeias decrescentes, com a ordem $\supsetneq^{*}$. Agora, é sempre possível associar objetos bem-fundados a ordinais, através de uma função posto.

Definição 2.2.6. Sejam $k<\omega, X$ um conjunto qualquer e $T$ uma árvore bem-fundada $\mathrm{em} \omega^{k} \times X$.

1. A função posto de $T$ é uma função $\rho_{T}: T \rightarrow O N$ definida por

$$
\rho_{T}(t)=\sup \left\{\rho_{\mathrm{T}}(\mathrm{s})+1 \mid \mathrm{s} \supsetneq * \mathrm{t}\right\} .
$$

2. A altura de $T$ é definida por

$$
\|T\|=\operatorname{im}\left(\rho_{\mathrm{T}}\right) .
$$

Os três fatos listados no lema a seguir são consequências simples das definições anteriores e de propriedades conhecidas sobre relações bem-fundadas. A seguir, destacamos três fatos que usaremos na nossa análise dos conjuntos analíticos e dos conjuntos $\boldsymbol{\Sigma}_{2}^{1}$.

Lema 2.2.7. Sejam $k<\omega, X$ um conjunto qualquer e $T$ uma árvore bem-fundada em $\omega^{k} \times X$.

1. $\rho_{T}(t)=0$ se, e somente se, $t$ é maximal em $\supsetneq^{*}$.

2. $\|T\|=\rho_{t}(\emptyset, \ldots, \emptyset)$.

3. $\rho_{T}(t)=\|T / t\|$.

Note que a função $\rho_{T}$ é um isomorfismo de ordem, quando restringimos o contradomínio à imagem de $\rho_{T}$. Isto é, $\rho_{T}: T \rightarrow \operatorname{im}\left(\rho_{T}\right)$ é um isomorfismo de ordem. Esse será outro conceito chave na nossa análise dos conjuntos analíticos e $\boldsymbol{\Sigma}_{2}^{1}$.

Definição 2.2.8. Sejam $k<\omega, X$ um conjunto qualquer e $T$ uma árvore em $\omega^{k} \times X$ bem-fundada. Dizemos que uma função $\rho: T \rightarrow$ ON é um isomorfismo de ordem se

$$
\forall s, t \in T\left(s \supsetneq{ }^{*} t \rightarrow \rho(s)<\rho(t)\right) .
$$

Lema 2.2.9. Sejam $k<\omega, X$ um conjunto qualquer e $T$ uma árvore em $\omega^{k} \times X$. Então $T$ é bem-fundada se, e somente se, existe um isomorfismo de ordem $\rho: T \rightarrow$ ON. Além disso, se tal $\rho$ existe, então $\rho_{T}(t) \leq \rho(t)$, para todo $t \in T$ e $\|T\| \leq \max \left\{\omega_{1},|\mathrm{X}|^{+}\right\}$.

No início dessa seção introduzimos a árvore $T_{x}$, onde $x \in \omega^{\omega}$ é elemento de um conjunto coanalítico, que por sua vez pode ser descrito a partir de um Esquema de Suslin usando a Operação A. Esse tipo de árvore, obtida a partir de elementos em subconjuntos de $\omega^{\omega}$, é introduzida no seguinte contexto geral. 
Definição 2.2.10. Sejam $k<\omega, l \leq k, X$ um conjunto qualquer, $T$ uma árvore em $\omega^{k} \times X e$ $w=\left(x_{0}, \ldots, x_{l-1}\right) \in\left(\omega^{\omega}\right)^{l}$. Podemos definir $T_{w}$ uma árvore em $\omega^{k-l} \times X$ da seguinte forma:

$$
T_{w} \doteq\left\{\left(t_{0}, \ldots, t_{k-l}\right) \mid \exists m<\omega\left(\left(x_{0}\left\lceil m, \ldots, t_{k}\left\lceil m, t_{0}, \ldots, t_{k-l}\right) \in T\right)\right\} .\right.\right.
$$

Consideraremos árvores $T_{w}$ com $w \in\left(\omega^{\omega}\right)^{k}$. O caso discutido no início da seção é quando $k=1$. Agora, provaremos o teorema que já discutimos anteriormente, sobre a representação dos conjuntos coanalíticos por árvores bem-fundadas.

Teorema 2.2.11 ([LS23]). Sejam $r \in \omega^{\omega}$ e $A \subseteq\left(\omega^{\omega}\right)^{k}$. Então $A$ é $\Pi_{1}^{1}(r)$ se, e somente se, existe uma árvore $T$ em $\omega^{k} \times \omega$ tal que para qualquer $w \in\left(\omega^{\omega}\right)^{k}$,

$$
w \in A \leftrightarrow T_{w} \text { é bem-fundada, }
$$

e a relação $\left\{(i, w) \mid s_{i} \in T_{w}\right\}$ é recursiva em $r$.

Demonstração. Suponha que existe uma árvore T em $\omega^{k} \times \omega$ tal que

$$
w \in A \leftrightarrow T_{w} \text { é bem-fundada, }
$$

e a relação $\left\{(n, w) \mid s_{n} \in T_{w}\right\}$ é recursiva em $r$.

Note que

$$
T_{w} \text { é bem-fundada } \leftrightarrow \forall^{1} y \exists^{0} n \exists^{0} m\left(s_{n}=y\left\lceil m \wedge s_{n} \notin T_{w}\right) .\right.
$$

Logo, $A \in \Pi_{1}^{1}(r)$.

Reciprocamente, suponha $A \in \Pi_{1}^{1}(r)$ e considere, pelo Lema 1.4.28, uma relação $R \subseteq \omega^{k+2}$ recursiva em $r$ tal que

$$
w \in A \leftrightarrow \forall^{1} y \exists^{0} m R(m, \bar{w}(m), \bar{y}(m)) .
$$

Podemos definir a árvore $T$ em $\omega^{k} \times \omega$ formada por todas as sequências $\left(s_{1}, \ldots, s_{k}, t\right)$ tais que $\forall^{0} i<\left|s_{1}\right|+1$

$$
\neg R\left(i,\left\langle s_{1}(i), \ldots, s_{1}(i-1)\right\rangle, \ldots,\left\langle s_{k}(i), \ldots, s_{k}(i-1)\right\rangle,\langle t(i), \ldots, t(i-1)\rangle\right) .
$$

Assim, $w \in A$ se, e somente se, $T_{w}$ é bem-fundada e $\left\{(n, w) \mid s_{n} \in T_{w}\right\}$ é recursiva em $r$.

Corolário 2.2.12. Sejam $r \in \omega^{\omega}$ e $A \subseteq\left(\omega^{\omega}\right)^{k}$. Então $A$ é $\Sigma_{1}^{1}(r)$ se, e somente se, existe uma árvore $T$ em $\omega^{k} \times \omega$ tal que para qualquer $w \in\left(\omega^{\omega}\right)^{k}$,

$$
w \in A \leftrightarrow T_{w} \text { é mal-fundada, }
$$

e a relação $\left\{(i, w) \mid s_{i} \in T_{w}\right\}$ é recursiva em $r$

Corolário 2.2.13. Seja $A \subseteq\left(\omega^{\omega}\right)^{k}$ um conjunto $\Sigma_{1}^{1}$. Então existe uma árvore $T$ em $\omega^{k} \times \omega$ tal que

$$
A=p[T]
$$

Demonstração. Usando o corolário anterior, seja $T$ árvore em $\omega^{k} \times \omega$ tal que $T_{w}$ é mal-fundada se, e somente se, $w \in A$. Basta notar que

$$
w \in p[T] \leftrightarrow \exists g((w, g) \in[T]) \leftrightarrow T_{w} \text { é mal-fundada. }
$$

O corolário anterior, junto com o Lema 2.2.5, fornece uma representação arbórea para conjuntos analíticos, i.e., um conjunto é analítico se ele é a projeção do conjunto dos ramos de uma árvore em 
$\omega^{k}$. Representações desse tipo são essenciais na análise dos conjuntos $\boldsymbol{\Sigma}_{n}^{1}$, como veremos na Seção 2.3 .

Definição 2.2.14. Sejam $A \subseteq\left(\omega^{\omega}\right)^{k}$ qualquer e $Y$ um conjunto qualquer. Dizemos que $A$ é $Y$ Suslin se existe uma árvore $T$ em $\omega^{k} \times Y$ tal que $A=p[T]$.

Com esta definição, provamos no Corolário 2.2.13 que os conjuntos analíticos são os conjuntos $\omega$-Suslin.

Encerramos essa seção com alguns resultados sobre absolutividade. Considerando que todas as noções envolvidas são absolutas para modelos transitivos de ZF, o seguinte teorema não deve ser tão surpreendente.

Teorema 2.2.15. Sejam $r \in \omega^{\omega}, \varphi \in \Sigma_{1}^{1}(r)$ e $M$ é um modelo transitivo de ZF tal que $r \in M$. Então $\varphi$ é absoluta para $M e \mathrm{~V}$.

Demonstração. Seja $T \in M$ uma árvore em $\omega^{k} \times \omega$ tal que

$$
\varphi(w) \leftrightarrow T_{w} \text { é mal-fundada. }
$$

- Seja $w \in M$ e suponha que $(\varphi(w))^{M}$. Então ( $T_{w}$ é mal-fundada $)^{M}$ e, portanto, $T_{w}$ é malfundada.

- Seja $w \in M$ e suponha $(\neg \varphi(w))^{M}$. Então $\left(T_{w} \text { é bem-fundada }\right)^{M}$ e, portanto, existe $f \in M$ tal que $\left(f: T_{w} \rightarrow \text { ON é isomorfismo de ordem }\right)^{\mathrm{M}}$. Segue que $f \in V$ e, pela absolutividade das noções envolvidas, vale $\varphi(w)$.

Corolário 2.2.16. Sejam $r \in \omega^{\omega}$, e $M, N$ modelos transitivos de ZF com $M \subseteq N$.

1. Se $\varphi \in \Sigma_{2}^{1}(r)$ e $\varphi^{M}$, então $\varphi^{N}$.

2. Se $\varphi \in \Pi_{2}^{1}(r)$ e $\varphi^{N}$, então $\varphi^{M}$.

Corolário 2.2.17. As seguintes fórmulas são absolutas para modelos transitivos de ZF $+\mathrm{DC}$ :
1. $x \in B_{c}$
2. $B_{c}=\emptyset$
3. $B_{c} \subseteq B_{d}$
4. $B_{c}=B_{d}$
5. $B_{e}=B_{c} \cup B_{d}$
6. $B_{e}=B_{c} \cap B_{d}$
7. $B_{e}=\omega^{\omega} \backslash B_{c}$
8. $B_{e}=B_{c} \Delta B_{d}$
9. $B_{c}=\bigcup_{n<\omega} B_{c_{n}}$

Demonstração. As fórmulas de 1. a 4. são $\Pi_{1}^{1}$ (Teoremas 2.1.7 e 2.1.8) e, portanto, absolutas para modelos transitivos de ZF + DC. Agora, se provarmos que a fórmula dos ítens 7. e 9. são absolutas, seguirá trivialmente todas as outras, pois $\cup$ pode ser definida a partir de " $\cup_{n<\omega}$ ", e " $\cap$ " e " $\Delta$ " podem ser definidos a partir de "U" e " $"$ ". Assim, seja $M$ um modelo transitivo de ZF+DC. 
7. Se $c \in M$ e $d \in \omega^{\omega}$ são tais que $d(0)=0$ e $u(d)=c$, então $d \in \mathrm{BC}, d \in M$ e $d$ codifica o conjunto $\omega^{\omega} \backslash A_{c}$ em $V$ e em $M$. Se $e \in M$, então

$$
B_{e}^{M}=\left(\omega^{\omega} \backslash B_{c}\right)^{M}=B_{d}^{M} \leftrightarrow B_{e}^{M}=B_{d}^{M} \leftrightarrow B_{e}=B_{d}
$$

9. Sejam $\left\{c_{n} \in \mathrm{BC} \mid n<\omega\right\}$ uma família de códigos em $M$ e $c \in \omega^{\omega}$ tais que $c(0)=1$ e $v_{i}(c)=c_{n}$ para todo $n<\omega$. Assim, $c \in \mathrm{BC}, c \in M$ e $c$ codifica o conjunto $\bigcup_{n<\omega} B_{c_{n}}$, tanto em $V$ quanto em $M$. Se $e \in M$, então

$$
B_{e}^{M}=\bigcup_{n<\omega} B_{c_{n}}^{M}=B_{c}^{M} \leftrightarrow B_{e}=B_{c} \leftrightarrow B_{e}=\bigcup_{n<\omega} B_{c_{n}} .
$$

\subsection{Representação arbórea de Shoenfield dos conjuntos $\Sigma_{2}^{1}$}

Como mencionado no início da seção anterior, todo conjunto analítico, ou coanalítico, pode ser escrito como a união (ou interseç̧ão) de $\aleph_{1}$ borelianos — esse é o Teorema 2.2.1. Em 1925 [Sie25], Sierpiński estendeu o Teorema 2.2.1 para o segundo nível da hierarquia projetiva: $\boldsymbol{\Sigma}_{2}^{1}$.

Teorema 2.3.1. Todo conjunto $\boldsymbol{\Sigma}_{2}^{1}$ pode ser escrito como a união de $\aleph_{1}$ borelianos.

Com isso, Sierpiński pôde concluir que todo conjunto $\boldsymbol{\Sigma}_{2}^{1}$ ou tem cardinalidade no máximo $\aleph_{1}$, ou possui um subconjunto perfeito e, portanto, tem cardinalidade $2^{\aleph_{0}}$. A prova de Sierpiński fez uso substancial do Axioma da Escolha. Em 1961 [Sho61] Shoenfield mostrou que os conjuntos $\boldsymbol{\Sigma}_{2}^{1}$ são $\omega_{1}$-Suslin, obtendo uma representação arbórea em $L$ para esses conjuntos, e a partir disso uma prova do Teorema 2.3.1 que dispensa o Axioma da Escolha.

Teorema 2.3.2 ([Sho61]). Sejam $r \in \omega^{\omega}$ e $A \subseteq\left(\omega^{\omega}\right)^{k}$ um conjunto $\Sigma_{2}^{1}(r)$. Então existe uma árvore $T \in L[r]$ em $\omega^{k} \times \omega_{1}$ tal que $A=p[T]$. Chamamos essa árvore $T$ de árvore de Schoenfield para A. Em particular, todo conjunto $\Sigma_{2}^{1}$ é $\omega_{1}$-Suslin.

Demonstração. Seja $A^{\prime}$ é um subconjunto $\Pi_{1}^{1}(r)$ de $\left(\omega^{\omega}\right)^{k+1}$ tal que $A=p A^{\prime}$. Pelo Teorema 2.2.11, existe uma árvore $T^{\prime}$ em $\omega^{k+1} \times \omega$ tal que

$$
w \in A^{\prime} \leftrightarrow T_{w}^{\prime} \text { é bem-fundada. }
$$

Primeiramente, note que trivialmente $T^{\prime} \in L[r]$. Agora, pelo Lema 2.2.9,

$$
w \in A^{\prime} \leftrightarrow \exists f\left(f: T_{w}^{\prime} \rightarrow \omega_{1} \text { é isomorfismo de ordem }\right) .
$$

De fato, basta tomar $f=\rho_{T_{w}^{\prime}}$, e então $\left\|T_{w}^{\prime}\right\| \leq \max \left\{\omega_{1},|\omega|^{+}\right\}=\omega_{1}$.

Considere agora a enumeração recursiva $\left(s_{n} \mid n<\omega\right)$ de $\omega^{<\omega}$ tal que $\left|s_{n}\right| \leq n$, para todo $n<\omega$. Podemos definir uma árvore $\hat{T}$ em $\omega^{k+1} \times \omega_{1}$ da seguinte forma:

$$
\hat{T}=\left\{\left(t_{0}, \ldots, t_{k}, u\right)|\forall i, j<| t_{0} \mid\left(s_{j} \subsetneq s_{i} \wedge\left(t_{0} \uparrow\left|s_{i}\right|, \ldots, t_{k} \uparrow\left|s_{i}\right|, s_{i}\right) \in T^{\prime} \rightarrow u(i)<u(j)\right)\right\} .
$$

Agora, se $f: T_{w}^{\prime} \rightarrow \omega_{1}$ é um isomorfismo de ordem, então a função $g: \omega \rightarrow \omega_{1}$ definida por

$$
g(n)= \begin{cases}f\left(s_{n}\right) & \text { se } s_{n} \in T_{w}^{\prime} \\ 0 & \text { caso contrário }\end{cases}
$$

é tal que $(w, g) \in[\hat{T}]$. Reciprocamente, se $g: \omega \rightarrow \omega_{1}$ é uma função tal que $(w, g) \in[\hat{T}]$, então a função $f: T_{w}^{\prime} \rightarrow \omega_{1}$ definida por $f\left(s_{n}\right)=g(n)$ é isomorfismo de ordem. Em qualquer caso, temos 


$$
\begin{aligned}
w \in A^{\prime} & \leftrightarrow \exists g \in \omega_{1}^{\omega}((w, g) \in[\hat{T}]) \\
\leftrightarrow & \leftrightarrow w \in p[\hat{T}] .
\end{aligned}
$$

Logo, $A^{\prime}=p[\hat{T}]$. Agora, a partir de $\hat{T}$, que é uma árvore em $\omega^{k+1} \times \omega$, podemos considerar $T \in L[r]$ uma árvore em $\omega^{k} \times\left(\omega \times \omega_{1}\right)$ tal que $p A^{\prime}=p[T]$. Assim, basta tomar em $L[r]$ uma bijeção $\omega \times \omega_{1} \leftrightarrow \omega_{1}$, que induz de forma natural uma árvore em $\omega^{k} \times \omega_{1}$ isomorfa a $T$. Logo, $A=p[T]$, com $T \in L[r]$.

Os seguintes teoremas serão usados na Seção 5.3.

Teorema 2.3.3 (Teorema da Decomposição de Shoenfield). Sejam $r \in \omega^{\omega}, A \subseteq\left(\omega^{\omega}\right)^{k} u m$ conjunto $\Sigma_{2}^{1}(r)$ e suponha que $\omega_{1}^{L[r]}=\omega_{1}$. Então existe uma família $\left\{B_{\alpha} \mid \alpha<\omega_{1}\right\}$ de borelianos de $\left(\omega^{\omega}\right)^{k}$ codificados em $L[r]$ tal que

$$
A=\bigcup_{\alpha<\omega_{1}} B_{\alpha}
$$

Demonstração. Considere $T$ árvore de Shoenfield para $A$ (Teorema 2.3.2), i.e., $T \in L[r]$ é uma árvore em $\omega^{k} \times \omega_{1}$ tal que $A=p[T]$.

Para cada $\beta<\omega_{1}$, seja $T_{\beta}=T \cap\left(\left(\omega^{<\omega}\right)^{k} \times \beta^{<\omega}\right)$ árvore induzida em $\omega^{k} \times \beta$ e note que $A=\bigcup_{\beta<\omega_{1}} p\left[T_{\beta}\right]$, pois $c f\left(\omega_{1}\right)>\omega$. Além disso, cada $T_{\beta} \in L[r]$ pois $T, \beta \in L[r]$.

A ideia é mostrar que cada $p\left[T_{\beta}\right]$ pode ser escrito como $\omega_{1}$-união de borelianos codificados em $L[r]$. Primeiramente, note que cada $p\left[T_{\beta}\right]$ é analítico pois $T_{\beta}$ é uma árvore em $\omega^{k} \times \beta$, e $\beta$ é um ordinal enumerável (portanto, $T_{\beta}$ é isomorfa a uma árvore em $\omega^{k} \times \omega$ ).

Assim, $\left(\omega^{\omega}\right)^{k} \backslash p\left[T_{\beta}\right]$ é coanalítico e, portanto, pelo Teorema 2.2.11:

$$
w \in\left(\omega^{\omega}\right)^{k} \backslash p\left[T_{\beta}\right] \leftrightarrow T_{w} \text { é bem fundada. }
$$

Agora, $T_{w}$ é uma árvore bem-fundada se, e somente se, para todo $\gamma<\omega_{1}$, ou $\left\|T_{w}\right\| \leq \gamma$ ou existe $s \in \omega^{<\omega}$ tal que $\left\|T_{w} / s\right\|=\gamma$. Assim,

$$
\begin{aligned}
\left(\omega^{\omega}\right)^{k} \backslash p\left[T_{\beta}\right]=\bigcap_{\gamma<\omega_{1}}\left\{w \in\left(\omega^{\omega}\right)^{k} \mid\right. & \left(T_{w} \text { é bem-fundada } \wedge\left\|T_{w}\right\| \leq \gamma\right. \\
& \left.\left.\vee \exists s \in \omega^{<\omega}\left(T_{w} / s \text { é bem-fundada } \wedge\left\|T_{w} / s\right\|=\gamma\right)\right)\right\} .
\end{aligned}
$$

Sendo assim, fixe $\beta<\omega_{1}$ e para cada $\gamma<\omega_{1}$ e naturalmente considere o conjunto

$$
\begin{aligned}
B_{\beta, \gamma}=\left\{w \in\left(\omega^{\omega}\right)^{k} \mid\right. & \left(T_{w} \text { é bem-fundada } \wedge\left\|T_{w}\right\| \leq \gamma\right. \\
& \left.\left.\vee \exists s \in \omega^{<\omega}\left(T_{w} / s \text { é bem-fundada } \wedge\left\|T_{w} / s\right\|=\gamma\right)\right)\right\} .
\end{aligned}
$$

Vamos mostrar que cada $B_{\beta, \gamma}$ é boreliano. Para isso, primeiro note que

$$
\begin{aligned}
B_{\beta, \gamma} & =\bigcup_{\delta \leq \gamma}\left\{w \in\left(\omega^{\omega}\right)^{k} \mid T_{w} \text { é bem-fundada } \wedge\left\|T_{w}\right\|=\delta\right\} \\
& \bigcup_{s \in \omega<\omega}\left\{w \in\left(\omega^{\omega}\right)^{k} \mid T_{w} / s \text { é bem-fundada } \wedge\left\|T_{w} / s\right\|=\gamma\right\} .
\end{aligned}
$$

Fixando também $\gamma<\omega_{1}$, para cada $\delta<\gamma$ e $s \in \omega^{<\omega}$, defina os conjuntos

- $C_{\beta, \gamma}^{\delta}=\left\{w \in\left(\omega^{\omega}\right)^{k} \mid T_{w}\right.$ é bem-fundada $\left.\wedge\left\|T_{w}\right\|=\delta\right\}$. 
- $C_{\beta, \gamma}^{s}=\left\{w \in\left(\omega^{\omega}\right)^{k} \mid T_{w} / s\right.$ é bem-fundada $\left.\wedge\left\|T_{w} / s\right\|=\gamma\right\}$.

Vamos mostrar que cada um desses conjuntos é boreliano. Faremos para os conjuntos da forma $C_{\beta, \gamma}^{s}$ e para $C_{\beta, \gamma}^{\delta}$ é análogo. Procedemos por indução transfinita:

- $C_{\beta, 0}^{s}$ é boreliano.

De fato, seja $w \in C_{\beta, 0}^{s}$. Temos que $T_{w} / s$ é bem-fundada e $\rho_{T}(s)=\left\|T_{w} / s\right\|=0$ e, portanto, $s$ é maximal na ordem $\supsetneq^{*}$. Assim, para todo $n<\omega, s^{\curvearrowleft} n \notin T_{w}$. Ou seja:

$$
C_{\beta, 0}^{s}=\bigcap_{n<\omega}\left\{w \in\left(\omega^{\omega}\right)^{k} \mid s^{\frown} n \notin T_{w}\right\},
$$

que é um boreliano.

- Suponha que para todo $\delta<\gamma$ e todo $s \in \omega^{<\omega}, C_{\beta, \delta}^{s}$ é boreliano. Então $C_{\beta, \gamma}^{s}$ é boreliano.

De fato, seja $w \in C_{\beta, \gamma}^{s}$. Então $T_{w} / s$ uma árvore bem-fundada com $\left\|T_{w} / s\right\|=\gamma$. Assim, para todo $n<\omega$, existe $\delta<\gamma$ tal que $\left\|\left(T_{w} / s\right) /(n)\right\|=\delta$, donde $\left(T_{w} / s\right) /(n)=T_{w} / s^{\frown}(n)$. Isto é, $w \in C_{\beta, \delta}^{s^{\Upsilon}(n)}$. Logo,

$$
C_{\beta, \gamma}^{s}=\bigcap_{n<\omega} \bigcup_{\delta<\gamma} C_{\alpha, \delta}^{s^{\frown(n)}} .
$$

Segue por indução que $C_{\beta, \gamma}^{s}$ é boreliano.

Pelos comentários acima, cada $B_{\beta, \gamma}$ é boreliano. Além disso, como a definição de cada $B_{\beta, \gamma}$ depende somente de $T_{\beta} \in L[r]$ e elementos de $\omega^{<\omega}$, podemos codificar cada um desses borelianos em $L[r]$.

Agora,

$$
p\left[T_{\beta}\right]=\bigcup_{\gamma<\omega_{1}}\left(\left(\omega^{\omega}\right)^{k} \backslash B_{\beta, \gamma}\right) .
$$

Logo,

$$
A=\bigcup_{\beta<\omega_{1}} \bigcup_{\gamma<\omega_{1}}\left(\left(\omega^{\omega}\right)^{k} \backslash B_{\beta, \gamma}\right) .
$$

Por fim, podemos tomar uma bijeção $\omega_{1} \times \omega_{1} \leftrightarrow \omega_{1}$ em $L[r]$, obtendo uma família $\left\{B_{\alpha} \mid \alpha<\omega_{1}\right\}$ de borelianos codificados em $L[r]$ tal que

$$
A=\bigcup_{\alpha<\omega_{1}} B_{\alpha} .
$$

Da mesma forma que as representações arbóreas para os conjuntos analíticos nos levam naturalmente a questões relativas a absolutividade para fórmulas $\Sigma_{1}^{1}(r)$, a representação arbórea de Shoenfield 2.3.2 para os conjuntos $\boldsymbol{\Sigma}_{2}^{1}$ permite um resultado análogo. No caso de fórmulas $\Sigma_{1}^{1}(r)$, a representação arbórea para conjuntos analíticos envolve apenas árvores em $\omega^{k} \times \omega$ e, portanto, envolve definições absolutas para modelos transitivos de ZF. Como a representação arbórea para conjuntos $\Sigma_{2}^{1}$ envolve árvores em $\omega^{k} \times \omega_{1}$, sendo $\omega_{1}$ não absoluto para modelos transitivos de ZF, a correção para absolutividade das fórmulas $\Sigma_{2}^{1}(r)$ é feita requerindo que os modelos envolvidos contenham $\omega_{1}$.

Teorema 2.3.4 ([Sho61]). Sejam $r \in \omega^{\omega}$ e $M$ modelo transitivo de $Z F$ tais que $\omega_{1} \cup\{r\} \subseteq M$. Então toda fórmula $\Sigma_{2}^{1}(r)$ é absoluta para $M$ e $V$. 
Demonstração. Seja $\varphi \in \Pi_{1}^{1}(r)$ e considere a fórmula $\psi \in \Sigma_{2}^{1}(r)$ como sendo a fórmula $\exists^{1} y \varphi$. Seja $T$ uma árvore em $\omega^{2} \times \omega$ tal que, para todo $x \in \omega^{\omega}$

$$
\psi(x) \leftrightarrow \exists^{1} y\left(T_{(x, y)}\right. \text { é bem-fundada). }
$$

Podemos assumir que $T^{M}=T$.

- Suponha $x \in M$ e $\psi(x)^{M}$.

Assim, existe $y \in M \cap \omega^{\omega}$ tal que $\left(T_{(x, y)} \text { é bem-fundada }\right)^{M}$. Assim, em $M$ existe um isomorfismo de ordem $\rho: T_{(x, y)} \rightarrow O N$. Segue que esse mapa também está em $V$, por argumentos canônicos de absolutividade.

- Suponha $x \in M$ e $\psi(x)$.

Como $\omega_{1} \cup\{r\} \subseteq M$, então $L[r] \subseteq M$. Usando a representação de Shoenfield para conjuntos $\Sigma_{2}^{1}(r)$, podemos tomar $T \in L[r]$ uma árvore em $\omega \times \omega_{1}$ tal que para todo $x \in \omega^{\omega}$,

$$
\psi(x) \leftrightarrow T_{x} \text { é mal-fundada. }
$$

Logo, não existe em $V$ um isomorfismo de ordem $T_{x} \rightarrow \omega_{1}$ e, portanto, esse mapa não pode existir em $M$ também.

\subsection{Como provar teoremas sobre borelianos usando jogos}

O estudo dos jogos infinitos e estratégias vencedoras para jogos infinitos tem origem na análise de jogos finitos. Um jogo infinito de dois jogadores com informação perfeita, que chamaremos apenas de jogo, é uma situação entre dois jogadores I e II, que jogam usando dois conjuntos $A$ e $X$. A seguir, vamos introduzir formalmente a noção de jogo infinito, bem como os elementos e conceitos principais associados a essa noção. A teoria geral dos jogos infinitos foi desenvolvida primeiramente por Gale e Stewart em 1953 [GS53].

Definição 2.4.1. Um jogo é um par $(X, A)$ onde:

- $X$ é um conjunto não vazio qualquer.

- $A \subseteq X^{\omega}$ é o que chamamos de conjunto payoff.

Denotamos esse par por $G_{X}(A)$.

Observação. Frequentemente denotaremos um jogo $G_{X}(A)$ apenas por $G(A)$, e $X$ ficará implícito pelo contexto.

Definição 2.4.2. Seja $G_{X}(A)$ um jogo.

1. Uma partida do jogo $G_{X}(A)$ é uma sequência $\left(x_{n}\right)_{n<\omega}$, onde a subsequência $\left(x_{2 n}\right)_{n<\omega}$ é a jogada do jogador I e a subsequência $\left(x_{2 n+1}\right)_{n<\omega}$ é a jogada do jogador II. Ilustramos uma partida da seguinte forma:

$$
\begin{array}{lllllll}
I & x_{0} & & x_{2} & & \cdots & \\
I I & & x_{1} & & x_{3} & & \cdots
\end{array}
$$


Isto é, uma partida é uma sequência $\left(x_{0}, x_{1}, x_{2}, x_{3}, \ldots\right) \in X^{\omega}$ tal que que o jogador I começa jogando $x_{0} \in X$ de modo que $\left(x_{0}\right) \in X$, em seguida o jogador II joga $x_{1} \in X$ de modo que $\left(x_{0}, x_{1}\right) \in X$, então o jogador I joga $x_{2} \in X$ de modo que $\left(x_{0}, x_{1}, x_{2}\right) \in X$, e assim por diante.

2. Dizemos que I vence o jogo $G_{X}(A)$ se $\left(x_{n}\right)_{n<\omega} \in A$. Caso contrário, dizemos que II vence o jogo.

Uma noção que está sempre associada à ideia de jogo é a de estratégia. Uma estratégia para um jogador é uma forma de decidir qual será a próxima jogada a partir das jogadas anteriores. Uma forma natural de pensar em uma estratégia é como uma função $\sigma: X^{<\omega} \rightarrow X$. Assim, se os movimentos do jogo até um dado momento foram $\left(x_{0}, x_{1}, x_{2}, x_{3}, \ldots, x_{2 i-1}\right)$, o jogador I joga $x_{2 i}=\sigma\left(x_{1}, x_{2}, x_{3}, \ldots, x_{2 i-1}\right)$.

Definição 2.4.3. Considere o jogo $G_{X}(A)$.

1. 1.1 Uma estratégia para o jogador I no jogo $G_{X}(A)$ é uma função

$$
\sigma: \bigcup_{n<\omega} X^{2 n} \rightarrow X
$$

1.2 Se o é uma estratégia para o jogador I, e $y=\left(y_{n}\right)_{n<\omega}$ é uma jogada do jogador II, definimos

$$
\sigma * y \doteq\left(x_{0}, y_{0}, x_{1}, y_{1}, \ldots, x_{i}, y_{i}, \ldots\right) .
$$

Onde a sequência $\left(x_{n}\right)_{n<\omega}$ é definida recursivamente da seguinte forma:

- $x_{0}=\sigma(\emptyset)$.

- $x_{i+1}=\sigma\left(x_{0}, y_{0}, \ldots, x_{i}, y_{i}\right)$.

Se $s \in X^{<\omega}$ é uma etapa da jogada do jogador II, $\sigma * s$ é a sequência finita com a definição esperada.

1.3 Dizemos que uma estratégia $\sigma$ é vencedora para $I$ se $\sigma * y \in A$, para todo $y \in X^{\omega}$.

2. 2.1 Uma estratégia para o jogador II no jogo $G_{X}(A)$ é uma função

$$
\tau: \bigcup_{n<\omega} X^{2 n+1} \rightarrow X
$$

2.2 Se $\tau$ uma estratégia para o jogador II e $x=\left(x_{n}\right)_{n<\omega}$ é uma jogada do jogador I, definimos

$$
x * \tau \doteq\left(x_{0}, y_{0}, x_{1}, y_{1}, \ldots, x_{i}, y_{i}, \ldots\right) .
$$

Onde a sequência $\left(y_{n}\right)_{n<\omega}$ é definida recursivamente da seguinte forma:

- $y_{0}=\sigma\left(x_{0}\right)$.

- $y_{i+1}=\sigma\left(x_{0}, y_{0}, \ldots, x_{i}, y_{i}, x_{i+1}\right)$.

Se $t \in X^{<\omega}, t * \tau$ tem a definição esperada.

2.3 Dizemos que uma estratégia $\tau$ é vencedora para II se $x * \tau \notin A$, para todo $x \in X^{\omega}$.

3. $G_{X}(A)$ é dito determinado se um dos jogadores, I ou II, possui uma estratégia vencedora. $\dashv$

Definição 2.4.4. Seja $A \subseteq \omega^{\omega}$. Dizemos que $A$ é determinado se $G_{\omega}(A)$ é um jogo determinado. $\dashv$

É natural então perguntar se todo jogo é determinado. Isso foi provado falso por Gale e Stewart em [GS53]. 
Teorema 2.4.5 ([GS53]). Existe um conjunto de reais que não é determinado.

Demonstração. Seja $A \subseteq \omega^{\omega}$ e considere o jogo $G_{\omega}(A)$. Então existe $\mathfrak{c}$ estratégias nesse jogo. Considere $\left(\sigma_{\alpha} \mid \alpha<\mathfrak{c}\right)$ uma $\mathfrak{c}$-enumeração de todas as estratégias possíveis do jogador I e $\left(\tau_{\alpha} \mid \alpha<\mathfrak{c}\right)$ uma c-enumeração de todas as estratégias possíveis do jogador II. Definiremos conjuntos $A=$ $\left\{a_{\alpha} \mid \alpha<\mathfrak{c}\right\}$ e $B=\left\{b_{\alpha} \mid \alpha<\mathfrak{c}\right\}$ disjuntos por recursão transfinita da seguinte forma. Suponha que já escolhemos $a_{\beta}$ e $b_{\beta}$ com $\beta<\alpha$. Considere o conjunto Plays ${ }_{\alpha} \doteq\left\{\sigma_{\alpha} * y \mid y \in \omega^{\omega}\right\}$ e note que $\mid$ Plays $_{\alpha} \mid=\mathfrak{c}$. Assim, podemos escolher $b_{\alpha} \in$ Plays $_{\alpha} \backslash\left\{a_{\beta} \mid \beta<\alpha\right\}$, digamos $b \doteq \sigma_{\alpha} * y$ para algum $y$. Analogamente, podemos escolher $a_{\alpha} \doteq z * \tau_{\alpha}$ para algum $z$ tal que $a_{\alpha} \notin\left\{b_{\beta} \mid \beta<\mathfrak{c}\right\}$. Sejam $A$ e $B$ como acima e note que $A \cap B=\emptyset$, por construção, e além disso, nem I nem II tem alguma estratégia vencedora.

Por outro lado, Gale e Stewart conseguiram provar que os conjuntos abertos (ou fechados) de reais são determinados. Esse resultado é o primeiro passo na direção de estabelecer a determinância para jogos com conjuntos definíveis de reais. Esse resultado é apresentado no contexto geral em que $X$ é um espaço topológico discreto, ao invés de simplesmente $\omega$.

Teorema 2.4.6 ([GS53]). Todo conjunto aberto (ou fechado) de reais é determinado.

Demonstração. Seja $A \subseteq \omega^{\omega}$ um aberto e considere o jogo $G_{\omega}(A)$. Para um conjunto qualquer $B \subseteq \omega^{\omega}$ e $s \in \omega^{<\omega}$, defina $B \doteq\left\{x \in \omega^{\omega} \mid s^{\wedge} x \in B\right\}$. Note que se I não tem uma estratégia vencedora no jogo $G_{\omega}(B / s)$ então para todo $i<\omega$, existe $j<\omega$ tal que I não tem estratégia vencedora no jogo $G_{\omega}\left(B / s^{\frown}(i, j)\right)$. De fato, suponha que existe $i<\omega$ tal que para todo $j<\omega$, I tenha uma estratégia vencedora $\sigma_{j}$ no jogo $G_{\omega}\left(B / s^{\frown}(i, j)\right)$. Então a seguinte estratégia seria vencedora para o jogador I no jogo $G_{\omega}(B / s)$ : I começa jogando $i$, e joga $i$ após cada jogada $j$ de II.

Agora assuma que o jogador I não tenha uma estratégia vencedora no jogo $G_{\omega}(A)$ e vamos construir recursivamente uma estratégia vencedora $\tau$ para II da seguinte forma: para a jogada $i_{0}$ do jogador I, o jogador II escolhe $i_{1}$ tal que o jogador I não possui estratégia vencedora no jogo $G_{\omega}\left(A /\left(i_{0}, i_{1}\right)\right)$; suponha que a estratégia para II está definida até a jogada $i_{2 n-2}$ do jogador I, i.e., o jogador II escolhe $i_{2 n-1}$ tal que o jogador I não tem estratégia vencedora no jogo $G_{\omega}\left(A /\left(i_{0}, i_{1}\right)^{\frown} \ldots \frown\left(i_{2 n-2}, i_{2 n-1}\right)\right)$; para a jogada $i_{2 n}$ do jogador I, o jogador II escolhe $i_{2 n+1}$ tal que I não tem estratégia vencedora no jogo $G_{\omega}\left(A /\left(i_{0}, i_{1}\right)^{\frown} \ldots \frown\left(i_{2 n-2}, i_{2 n-1}\right)^{\frown}\left(i_{2 n}, i_{2 n+1}\right)\right)$. Definimos uma estratégia $\tau$ tal que para qualquer jogo parcial $s \in \bigcup_{n<\omega} \omega^{2 n}$ de acordo $\operatorname{com} \tau$, o jogador I não tem estratégia vencedora no jogo $G_{\omega}(A / s)$. Vamos mostrar que essa estratégia $\tau$ é vencedora para II.

Suponha que $\tau$ não é uma estratégia vencedora para II. Então existe $x \in \omega^{\omega}$ um jogo tal que II jogou de acordo com $\tau$ mas $x \in A$. Como $A$ é aberto, existe $n<\omega$ tal que $\mathcal{O}_{x \nmid 2 n} \subseteq A$. Mas então, qualquer estratégia para I no jogo $G_{\omega}(A / x\lceil 2 n)$ seria vencedora. Absurdo. Logo, $\tau$ é uma estratégia vencedora para II, como queríamos.

Em [GS53] Gale e Stewart perguntaram se todos os borelianos são determinados. Essa pergunta só foi respondida duas décadas depois por Martin (veja o Teorema 2.4.12).

Em 1962 [MS62] Mycielski e Steinhaus introduziram o Axioma da Determinância, que afirma que todo conjunto de reais é determinado.

Definição 2.4.7. O Axioma da Determinância (AD) é a afirmação

"todo conjunto de reais é determinado."

A relação entre jogos infinitos e regularidade já havia sido especulada por Mazur para o caso da propriedade de Baire. Essa relação foi confirmada por Banach em 1935 (veja [Mau81] para essas informações). O jogo de Banach-Mazur é apresentado a seguir, adaptado para a nossa linguagem. Para uma demonstração desse teorema, veja a Proposição 27.3 de [Kan08] 
Definição 2.4.8. Seja $A \subseteq \omega^{\omega}$. O jogo de Banach-Mazur $G^{* *}(A)$ é definido da seguinte forma:

- Ambos os jogadores jogam com sequências finitas não-vazias de números naturais, como ilustrado abaixo.

$\begin{array}{lllllll}I & s_{0} & & s_{2} & & \cdots & \\ I I & & s_{1} & & s_{3} & & \end{array}$

- O jogador I ganha no jogo $G^{* *}(A)$ se, e somente se, $x \doteq s_{0}^{\frown} s_{1}^{\frown} s_{2}^{\imath} \cdots \in A$.

Teorema 2.4.9 (Teorema de Banach-Mazur, veja [Mau81]). Seja $A \subseteq \omega^{\omega}$.

1. O jogador I tem uma estratégia vencedora em $G^{* *}(A)$ se, e somente se, A é um conjunto magro.

2. O jogador II tem uma estratégia vencedora em $G^{* *}(A)$ se, e somente se, existe $s \in \omega^{<\omega}$ tal que $\mathcal{O}_{s} \backslash A$ é magro.

A partir disso, é possível mostrar que $A \subseteq \omega^{\omega}$ é Baire mensurável se o jogo $G^{* *}\left(A \backslash \mathcal{O}_{A}\right)$ é determinado, onde $\mathcal{O}_{A} \doteq \bigcup\left\{\mathcal{O}_{s} \mid s \in \omega^{<\omega} \wedge \mathcal{O}_{s} \backslash A\right.$ é magro\} (veja o Corolário 27.4 de [Kan08]).

$\mathrm{O}$ primeiro resultado relacionando regularidade com jogos, depois do Teorema 2.4.9, foi o $\mathrm{Te}$ orema de Davis, de 1964 [Dav64], onde Davis formulou um jogo infinito e relacionou sua determinância com a propriedade do conjunto perfeito.

Definição 2.4.10. Seja $A \subseteq \omega^{\omega}$. O jogo de Davis $G^{*}(A)$ é definido da seguinte forma:

- O jogador I joga com sequências finitas não vazias de números naturais (i.e., elementos de $\left.\omega^{<\omega}\right)$. O jogador II joga com números naturais, como ilustrado abaixo.

$\begin{array}{lllllll}I & s_{0} & & s_{1} & & \cdots & \\ I I & & n_{1} & & n_{2} & & \cdots\end{array}$

- O jogador I ganha no jogo $G^{*}(A)$ se, e somente se,

(a) $\forall i \geq 1\left(s_{i}(0) \neq n_{i}\right) e$

(b) $x \doteq s_{0} \widehat{s_{1}} \overbrace{2}^{\imath} \cdots \in A$.

Teorema 2.4.11 (Teorema de Davis [Dav64]). Seja $A \subseteq \omega^{\omega}$.

1. Se o jogador I tem uma estratégia vencedora em $G^{*}(A)$, então A possui um subconjunto não vazio perfeito.

2. Se o jogador II tem uma estratégia vencedora em $G^{*}(A)$, então A é enumerável. 
Provaremos o teorema acima na Seção 4.3. Para a propriedade de Lebesgue, o primeiro argumento via jogos foi dado em 1964 [MŚ64] por Mycielski e Swierczkowski (veja a pg. 375 em [Kan08]). Juntando todos esses resultados, AD implica que todo conjunto de reais é Baire mensurável, é Lebesgue mensurável e tem a propriedade do conjunto perfeito.

Nos década 60 e no começo da década de 70 foram provados diversos teoremas usando a determinância de certos jogos, inclusive teoremas sobre a existência de certos cardinais grandes. Embora a teoria descritiva dos conjuntos tenha tido considerável avanço através de técnicas de determinância para jogos infinitos, até 1975 ainda não era conhecida uma prova da determinância para o caso básico em que os jogos têm como payoff um conjunto boreliano de reais. Para uma prova do seguinte teorema, veja o Capítulo 20 de [Kec95].

Teorema 2.4.12 (Teorema da Determinância Boreliana [Mar75]). Todo conjunto boreliano de reais é determinado.

O Teorema da Determinância Boreliana, da forma como enunciamos, fala apenas sobre jogos do tipo $G_{\omega}(A)$, onde $A \subseteq \omega^{\omega}$. Então a princípio a determinância de jogos como $G^{* *}((A)$ (que é um jogo da forma $\left.G_{\omega<\omega}(A)\right)$ e $G^{*}(A)$ (que também é um jogo da forma $G_{\omega<\omega} \cup \omega(A)$ ) não está assegurada por esse teorema nos casos em que $A$ é boreliano. Para resolver esse problema, é preciso codificar (recursivamente) esses jogos e construir jogos correspondentes da forma $G_{\omega}(A)$, de modo que a determinância para esses jogos codificados seja suficiente para assegurar a determinância dos jogos $G^{* *}(A)$ e $G^{*}(A)$. Em geral, esse processo é possível, mas não nos preocuparemos com isso nesse texto.

Encerramos esta seção fazendo alguns comentários sobre a força de consistência de AD e outros axiomas para determinância de jogos. Primeiramente, é natural perguntar se é possível generalizar o Teorema 2.4.12 para conjuntos analíticos, coanalíticos, etc. A determinância para os conjuntos $\Pi_{1}^{1}$ já implica na existência de um inacessível:

Teorema 2.4.13. Se todo conjunto $\Pi_{1}^{1}$ de reais é determinado, então $\forall r \in \omega^{\omega}\left(\aleph_{1}^{\mathrm{L}[r]}<\aleph_{1}\right)$.

PD é o axioma que diz que todos os conjuntos projetivos são determinados. Sabe-se que ZF + AD tem a força de consistência de ZFC + "existem infinitos cardinais de Woodin", e ZFC + PD tem a força de consistência de ZFC $+\forall n>0$ (existe um modelo interno com $n$ cardinais de Woodin). 


\section{Capítulo 3}

\section{Teoria geral do forcing}

\subsection{Forcing: uma abordagem heurística}

"O fato é que uma ideia nova leva a outra, e essa a uma terceira e assim por diante ao longo do tempo, até que alguém, a quem nenhuma dessas ideias era original, combina todas essas ideias, e produz o que é chamado de nova invenção."

[Jef13]

A abordagem de Cohen consistia em começar com um modelo $M$ de ZF e adicionar um conjunto $G$, que teria alguma nova propriedade desejada. Cohen percebeu que deveria fazer isso de um jeito "minimal", a fim de que a estrutura resultante ainda fosse um modelo de ZF, então precisou pensar sobre condições tanto sobre o modelo $M$, quanto sobre o objeto $G$.

Como noções básicas em teoria dos conjuntos são preservadas para $\in$-modelos transitivos, Cohen definiu a noção de modelo canônico como sendo um modelo para ZF ou ZFC com a relação $\in$. Ele postulou o "Axioma M" como sendo a afirmação "existe um modelo canônico para ZFC". Porém, a existência de um modelo canônico não pode ser provada em ZFC, nem mesmo assumindo ZFC + Con(ZFC).

Definição 3.1.1. Um modelo para a teoria dos conjuntos é dito canônico se é um modelo com a relação $\in$ restrita ao seu domínio.

Teorema 3.1.2. Se $\mathrm{ZFC}+\mathrm{Con}(\mathrm{ZFC}) \vdash \exists M(M$ é modelo canônico de $\mathrm{ZFC})$ então $\mathrm{ZFC}$ é inconsistente.

Demonstração. Assuma ZFC + Con(ZFC). Seja $M$ modelo canônico de ZFC e defina a teoria $T \doteq \mathrm{ZFC}+\mathrm{Con}(\mathrm{ZFC})$. Como $\in$ é bem-fundada e extensional em $M$, então $\pi_{M, \in}: M \rightarrow \bar{M}$ o colapso transitivo de $(M, \in)$ sobre $(\bar{M}, \in)$ é um isomorfismo e, portanto, $\bar{M} \models$ ZFC. Por outro lado, como Con(ZFC) é uma fórmula aritmética, então é absoluta para modelos transitivos de ZFC. Logo, também temos $\bar{M} \models$ Con(ZFC) e, portanto, $\bar{M} \models T$. Segue agora pelo Teorema da Completude de Gödel que

$$
T \vdash \operatorname{Con}(T) .
$$

O que contraria o Teorema da Incompletude Gödel.

Assim, Cohen introduziu o Axioma $\mathrm{M}$ e fez algumas especulações sobre por que esse axioma é "verdadeiro", em algum sentido. No entanto, as provas de independência são todas da forma "ZFC $\vdash \mathrm{Con}(\mathrm{ZFC}) \rightarrow \mathrm{Con}(\mathrm{ZFC}+\varphi)$ ", e pelo que argumentamos acima, Con(ZFC) é estritamente mais fraco do que o Axioma M. Veremos mais adiante como corrigir esse problema.

Além dessa limitação sobre os modelos de $M$, Cohen também impôs uma limitação sobre as possíveis cardinalidades para modelos em que podem valer $\neg \mathrm{CH}$.

Teorema 3.1.3 ([Coh08]). Suponha ZF consistente e seja M é um modelo canônico não enumerável de ZFC. Então 
1. $M \models \omega^{\omega} \subseteq L$

2. $M \models \mathrm{CH}$

Demonstração. Primeiro, note que podemos assumir $\mathrm{ZF}+\mathrm{V}=\mathrm{L}$, pois $\mathrm{Con}(\mathrm{ZF}) \rightarrow \operatorname{Con}(\mathrm{ZF}+\mathrm{V}=$ L). Assim, assuma ZF $+\mathrm{V}=\mathrm{L}$ e seja $M$ um modelo canônico de ZFC.

1. Seja $\alpha \doteq \sup (M \cap \mathrm{ON})$. Vamos verificar que $\alpha$ é não enumerável. Suponha que $\alpha$ é enumerável. Então pela absolutividade da função posto $(\rho)$, e pela não enumerabilidade de $M$, existe algum $\beta<\alpha$ tal que $V_{\gamma} \subseteq M$ é não enumerável. Agora, usando AC em $M$, isso significa que existe uma boa-ordem para $V_{\beta}$ em $M$ e, portanto, existe um ordinal não enumerável em $M$, o que contradiz $\alpha$ ser enumerável. Logo, $\alpha$ é não enumerável e, portanto, $N$ contém todos os ordinais enumeráveis. $\mathrm{De} \mathrm{V}=\mathrm{L}$ sabemos que todo número real é construtível a partir de um ordinal enumerável, logo, relativamente a $M$ todo número real é construtível.

2. Basta observar que $\mathrm{ZF}+\omega^{\omega} \subseteq \mathrm{L} \vdash \mathrm{CH}$ e $M$ como acima satisfaz $\mathrm{ZF}+\omega^{\omega} \subseteq \mathrm{L}$.

Com essa simples observação, concluímos que não é possível obter um modelo canônico não enumerável em que não vale $\mathrm{CH}$. Como mencionado anteriormente, Skolem já especulava sobre a possibilidade de se começar com um certo modelo para a teoria dos conjuntos e adicionar subconjuntos de $\omega$, a fim de variar a cardinalidade de $2^{\aleph_{0}}$, mas admitiu que isso seria potencialmente muito difícil de ser feito (veja a página 4).

Há diversas abordagens em textos introdutórios de forcing que solucionam os problemas dos Teoremas 3.1.2 e 3.1.3 (Veja, por exemplo, [Kun14]). Essas abordagens variam principalmente em gosto pessoal, e por esse motivo foi escolhida a abordagem que será apresentada, em que construimos uma extensão da linguagem $\mathcal{L}_{\in}$ por uma constante $M$, e uma extensão conservativa $\mathrm{ZFC}_{M}$, de ZFC. Essa ideia é usada em textos como [Wea14], e mencionada em apêndice em [Kun14].

Antes de tudo, enunciaremos o Teorema da Reflexão. O Teorema da Reflexão é na verdade um esquema de teoremas, ele afirma para cada fórmula $\varphi$ de $\mathrm{ZF}(\mathrm{C})$ pode ser refletida para dentro de um modelo canônico de ZFC. Montague em 1956 [Mon56] e em 1961 [Mon61] usou o Teorema da Reflexão para investigar axiomatizações finitas para a teoria dos conjuntos, e Levy em 1960 [Lév60a, Lev60b] aplicou o Teorema da Reflexão para ZF para caracterizar certas propriedades de cardinais Mahlo, o primeiro cardinal grande acima de inacessível.

Teorema 3.1.4 ([Mon56, Mon61], [Lév60a, Lev60b]). Para toda sentença $\varphi$ de $\mathcal{L}_{\in}, \mathrm{ZF}$ prova que existe um conjunto transitivo enumerável $M$ tal que

$$
\varphi \leftrightarrow \varphi^{M} .
$$

Definição 3.1.5. Sejam $M$ um novo símbolo de constante, $\mathcal{L}_{\in}(M) \doteq\{\in, M\}$ a linguagem da teoria dos conjuntos expandida pela constante $M$ e a teoria

$$
\mathrm{ZFC}_{M} \doteq Z F C \cup\{M \text { é enumerável e transitivo }\} \cup\left\{\varphi^{M} \mid \varphi \in \mathrm{ZFC}\right\}
$$

Teorema 3.1.6. $\operatorname{Con}(\mathrm{ZFC}) \leftrightarrow \operatorname{Con}\left(\mathrm{ZFC}_{M}\right)$.

Teorema 3.1.7. Seja $\varphi$ uma sentença da linguagem $\mathcal{L}_{\in}$. Suponha que, em $\mathrm{ZFC}_{M}$, conseguimos provar que existe $N$ um conjunto transitivo enumerável, que vale $\varphi^{N}$ e, além disso, valem todos os axiomas de ZFC. Então

$$
\mathrm{Con}(\mathrm{ZFC}) \rightarrow \mathrm{Con}(\mathrm{ZFC}+\varphi)
$$


Demonstração. Suponha $\mathrm{ZFC}_{M}$ inconsistente e sejam $\psi$ uma $\mathcal{L}_{\in}(M)$-fórmula, $\varphi_{1}, \ldots, \varphi_{n}$ axiomas de ZFC tais que

$$
\varphi_{1}^{M} \wedge \cdots \wedge \varphi_{n}^{M} \vdash \psi \wedge \neg \psi
$$

Como $\varphi_{1} \wedge \cdots \wedge \varphi_{n}$ é um teorema de ZFC, então pelo Teorema da Reflexão 3.1.4, existe uma prova em ZFC de que existe $N$ um modelo transitvo numerável satisfazendo $\varphi_{1} \wedge \cdots \wedge \varphi_{n}$, donde $\varphi_{1}^{N} \wedge \cdots \wedge \varphi_{n}^{N}$ é uma prova de $\left.\left.\psi\right|_{M=N} \wedge \neg \psi\right|_{M=N}$, i.e., produz uma contradição como em $\psi \wedge \neg \psi$, mas substituindo as ocorrências de $M$ por $N$. Logo, ZFC é inconsistente.

Até o fim da seção:

Estamos trabalhando na teoria $\mathrm{ZFC}_{M}$.

O seguinte trecho, retirado de $\left[\mathrm{C}^{+} 02\right]$, deve nortear a nossa discussão até o fim dessa seção.

"Então começamos com um modelo canônico enumerável $M$, e desejamos adicionar novos elementos e ainda obter um modelo. Uma decisão importante é não adicionar novos ordinais. A adição mais simples que se pode fazer é adicionar um conjunto de inteiros. (Aliás, esse problema de como adicionar um conjunto de inteiros a um modelo foi apontado por Skolem em suas observações gerais sobre como os axiomas falham para caracterizar o universo dos conjuntos unicamente.) Agora, é trivialmente possível adicionar um elemento que já está em $M$. Para testar a intuição, deve-se tentar adicionar a $M$ um elemento que não possui nenhuma propriedade específica, i.e., algo parecido com a adição de uma variável a um corpo. Chamei esse elemento de 'genérico'. Agora o problema é tornar precisa a definição de elemento genérico. Se for possível adicionar tal elemento, então haverá um método para adicionar vários e então criar vários modelos diferentes com várias propriedades." $\quad\left[\mathrm{C}^{+} 02\right]$

Dados conjuntos quaisquer $I, J \in M$, onde $I$ é infinito e $J \neq \emptyset$, nossa tarefa é conseguir construir um modelo $N \supseteq M$ em que existe uma função sobrejetora $f: I \rightarrow J$. Seguindo essa ideia de aproximar esse objeto $f$ por pedaços de informações sobre ele que vivem em $M$, considere $G \doteq\left\{p \subseteq f|| p \mid<\aleph_{0}\right\}$. Por absolutividade, todas as condições em $G$ pertencem a $M$, assim é natural considerar $\mathbb{P}=\operatorname{Fn}(I, J)$ o conjunto de funções finitas de $I$ em $J$ (i.e., se $p \in \mathbb{P}, \operatorname{dom}(p) \subseteq I$ e $|p| \leq \aleph_{0}$ ), e assim $G \subseteq \mathbb{P}$ e $\mathbb{P} \in M$. Conseguimos assim isolar algumas propriedades sobre $G$ e $f$ :

- Trivialmente $\emptyset \in G$, o que não nos dá nenhuma informação sobre $G$;

- Se $p \in G$ e $q \subseteq p$ então $q \in G$. Certamente $p$, que é uma extensão de $q$, nos dá mais informação sobre $G$. Também dizemos que $p$ é mais forte que $q$;

- Se $p, q \in G$, então $p \cup q \in G$.

Note que, dos comentários acima, temos uma ordem natural em $\mathbb{P}$. Na nossa ordem, convencionaremos que o menor é o mais forte, e com essa convenção, o conjunto $G$ como acima é simplesmente um filtro.

Definição 3.1.8. Uma noção de forcing é uma ordem parcial $(\mathbb{P}, \leq, \mathbb{1})$, onde $\mathbb{1}$ é o maior elemento de $\mathbb{P}$. Os elementos de $\mathbb{P}$ são ditos condições. Além disso, se $p, q \in \mathbb{P}$, dizemos que

1. $p$ é mais forte que $q$, ou $p$ estende $q$, se $p \leq q$.

2. $p$ e q são compatíveis, e denotamos $p \| q$, se existe $r \in \mathbb{P}$ tal que $r \leq p$ e $r \leq q$.

3. $p$ e q são incompativeis se não são compativeis, e denotamos por $p \perp q$.

Frequentemente diremos simplesmente "P é uma noção de forcing", e a ordem com o elemento máximo ficarão implícitos. 
Definição 3.1.9. Seja $\mathbb{P}$ uma noção de forcing. Dizemos que $G \subseteq \mathbb{P}$ é um filtro se

- $\mathbb{1} \in G$,

- para todos $p, q \in G$, existe $r \in G$ tal que $r \leq p$ e $r \leq q$ e

- para todo $p \in G$ e $q \in \mathbb{P}$, se $p \leq q$ então $q \in G$.

Ainda precisamos impor condições sobre $G$ a fim de que $\operatorname{dom}(f)=I$ e $\operatorname{im}(f)=J$ também.

- Para que $\operatorname{dom}(f)=I$, é suficiente que para todo $x \in I$, exista $p \in G$ tal que $x \in \operatorname{dom}(p)$, i.e., para todo $x, G \cap\{p \in \mathbb{P} \mid x \in \operatorname{dom}(p)\} \neq \emptyset$.

- Para que $\operatorname{im}(f)=J$, é suficiente que para todo $y \in J$, exista $p \in G$ tal que $y \in \operatorname{im}(p)$, i.e., para todo $y, G \cap\{p \in \mathbb{P} \mid y \in \operatorname{im}(p)\} \neq \emptyset$.

Note que por conta do primeiro caso que pedimos que $I$ fosse infinito. Agora, os conjuntos $D_{x} \doteq\{p \in \mathbb{P} \mid x \in \operatorname{dom}(p)\}$ e $D_{y} \doteq\{p \in \mathbb{P} \mid y \in \operatorname{im}(p)\}$ são o que chamamos de densos (na verdade a definição de conjunto denso foi dada por Solovay).

Definição 3.1.10. Sejam $\mathbb{P}$ é uma noção de forcing. Dizemos que $D \subseteq \mathbb{P}$ é denso em $\mathbb{P}$ se para todo $p \in \mathbb{P}$, existe $q \in D$ tal que $q \leq p$.

Definição 3.1.11. Sejam $\mathbb{P} \in M$ uma noção de forcing e $G \subseteq \mathbb{P}$ um filtro. Dizemos que $G$ é $\mathbb{P}$-genérico sobre $M$ se

$$
G \cap D \neq \emptyset
$$

para todo denso $D \in M$.

A primeira versão do forcing consistia em construir uma hierarquia ramificada a partir desse modelo canônico transitivo e enumerável. Primeiro, é importante observamos que, para algum ordinal $\beta, M=\bigcup_{\alpha<\beta} V_{\alpha} \cap M$, pois os ordinais de $M$ coincidem com um seguimento inicial de algum $\beta$. Assim, Cohen procedeu definindo um sistema de termos para denotar os elementos do novo modelo, trabalhando em uma linguagem ramificada. Essa ideia é descrita sucintamente a seguir. Para cada $x \in M$ consideramos $\dot{x}$ uma constante correspondente; consideramos $\dot{G}$ uma nova constante (para denotar o filtro genérico) e para cada $\alpha<\beta$ consideramos quantificadores $\exists_{\alpha}$ e $\forall_{\alpha}$. Feito isso, a hierarquia de termos é definida como da seguinte forma:

- $\dot{M}_{0}=\{\dot{G}\}$;

- $M_{\alpha+1}$ é a coleção de termos $\dot{x}$ tais que $x \in V_{\alpha} \cap M$ e termos correspondentes a fórmulas que permitem parâmetros a partir de $\dot{M}_{\alpha}$ e dos quantificadores $\forall_{\alpha}$ e $\exists_{\alpha}$;

- $\dot{M}_{\gamma} \doteq \bigcup_{\delta<\gamma} \dot{M}_{\delta}$, se $\gamma<\beta$ é um ordinal limite.

É importante observar que essa linguagem ramificada é formalizável em $M$ através de uma codificação de símbolos (argumento que já apareceu no trabalho de Gödel). Assim, dado algum $G$ fora do modelo $M, M \doteq \bigcup_{\alpha<\beta} M_{\alpha}[G]$ fica determinado interpretando os termos da linguagem ramificada, $\dot{x}$ é interpretado pelo $x$ de $M$ e $\dot{G}$ é interpretado por $G$ de modo que

- $M_{0}[G]=\{G\}$

- $M_{\alpha+1}$ é o conjunto dos conjuntos em $V_{\alpha} \cap M$ e conjuntos interpretando os termos correspondentes às fórmulas como subconjuntos definíveis de $M_{\alpha}[G]$, com os quantificadores $\exists_{\alpha}$ e $\forall_{\alpha}$ variando sobre o domínio;

- $M_{\gamma}<\beta \doteq \bigcup_{\delta<\gamma} M_{\delta}[G]$. 
As abordagens modernas de forcing seguem a apresentação não ramificada de Shoenfield, apresentada em 1971 [Sho71]. Shoenfield conseguiu adicionar o filtro genérico a um modelo canônico transitivo e enumerável de ZFC sem usar essa ideia de linguagem ramificada.

Definição 3.1.12. Sejam $\mathbb{P} \in M$ uma noção de forcing e $G \neq \emptyset$ um conjunto qualquer.

1. Definimos a relação $\in_{G} \subseteq M \times M$ da seguinte forma:

$$
x \in_{G} y \leftrightarrow \exists p \in G((x, p) \in y) .
$$

2. Considere $\pi_{M, G}: M \rightarrow \operatorname{im}\left(\pi_{M, G}\right)$ o colapso transitivo de $\left(M, \in_{G}\right)$ sobre a imagem. Então definimos a estrutura $\in$-transitiva

$$
M[G] \doteq \operatorname{im}\left(\pi_{M, G}\right) .
$$

Se $x \in M$, escreveremos $x^{G}$ ao invés de $\pi_{M, G}(x)$.

3. Dizemos que $\pi_{M, G}^{-1}(x)$ é o conjunto dos $\mathbb{P}$-nomes para $x$.

Na definição anterior, fica implícito que a relação $\in_{G}$ é uma relação bem-fundada em $M$ (segue trivialmente do fato de $\in$ ser uma relação bem-fundada). Como queremos um modelo para ZF(C) que tenha mais fórmulas verdadeiras, obviamente a relação $\epsilon_{G}$ não pode ser extensional, pois caso contrário $M$ e $M[G]$ seriam estruturas isomorfas. De fato, $\epsilon_{G}$ não é extensional, e o colapso transitivo não é injetor. Logo, a pré-imagem de cada $x \in M[G]$ pode ser um conjunto com mais de um elemento. Dizemos que $\pi_{M, G}^{-1}(x)$ é o conjunto dos $\mathbb{P}$-nomes para $x$.

Com essa forma de adicionar um objeto qualquer ao modelo $M$, resta provar que um filtro genérico sobre $M$ de fato existe. Cohen queria que qualquer sentença verdadeira no modelo $M[G]$ fosse decidida por alguma afirmação definível em $M$. Ainda que o genérico $G$ não exista em $M$ ele é composto por certas condições que existem em $M$, que de certa forma aproximam $G$, e essas condições poderiam forçar (em analogia para implicar) a fórmula $\varphi$ a ser verdadeira em $M[G]$. Mais precisamente, para uma sentença $\varphi$, Cohen queria decidir se $\varphi$ é verdadeira em $M[G]$ verificando se para alguma condição $p, p \in G$, sendo a fórmula " $p \in G$ " expressável na linguagem ramificada de Cohen (definida em $M$ ). Quando isso é possível, dizemos que $p$ força $\varphi$, e denotamos por $p \Vdash \varphi$.

Primeiro, vamos ver que para qualquer condição $p$, sempre existe um filtro $\mathbb{P}$-genérico sobre $M$ que contém $p$. Depois, vamos definir os conceitos dos comentários do último parágrafo.

Teorema 3.1.13. Sejam $\mathbb{P}$ uma noção de forcing e $p \in \mathbb{P}$. Então existe $G$ um filtro $\mathbb{P}$-genérico sobre $M$ tal que $p \in G$.

Demonstração. Sejam $\left(D_{n}\right)_{n<\omega}$ uma enumeração de todos os densos de $\mathbb{P}$ que estão em $M$. Recursivamente defina uma sequência $\left(p_{n}\right)_{n<\omega}$ de elementos em ordem decrescente de modo que $p_{n} \in D_{n}$, para todo $n<\omega$, da seguinte forma:

- $p_{0} \in D_{0}$ é tal que $p_{0} \leq p$;

- $p_{n+1} \in D_{n+1}$ é tal que $p_{n+1} \leq p_{n}$.

As escolhas acima são possíveis usando a densidade de cada $D_{n}$. Agora seja

$$
G \doteq\left\{q \in \mathbb{P} \mid \exists n<\omega\left(p_{n} \leq q\right)\right\} .
$$

É fácil verificar que $p \in G, G$ é filtro e é $\mathbb{P}$-genérico sobre $M$.

Definição 3.1.14. Sejam $\mathbb{P}$ uma noção de forcing, $\varphi\left(v_{1}, \ldots, v_{n}\right)$ uma $\mathcal{L}_{\in}$-fórmula, $p \in \mathbb{P}$ e $x_{1}, \ldots, x_{n} \in$ $M$. Dizemos que $p$ força $\varphi\left(x_{1}, \ldots, x_{n}\right)$, e denotamos $p \Vdash \varphi\left(x_{1}, \ldots, x_{n}\right)$ se

$$
\forall G\left(G \text { é filtro } \mathbb{P} \text {-genérico sobre } M \wedge p \in G \rightarrow \varphi^{M[G]}\left(x_{1}^{G}, \ldots, x_{n}^{G}\right)\right) .
$$


Como discutimos acima, para uma noção de forcing $\mathbb{P}$ e para cada fórmula $\varphi\left(v_{1}, \ldots, v_{n}\right)$ de $\mathcal{L}_{\epsilon}$, queremos encontrar uma fórmula $\bar{\varphi}\left(v, v_{1}, \ldots, v_{n}\right)$ de $\mathcal{L}_{\in}$ satisfazendo o que é chamado de Lema da Verdade: Para todo $G$ filtro $\mathbb{P}$-genérico sobre $M$ e para todos $x_{1}, \ldots, x_{n} \in M$

$$
\varphi^{M[G]}\left(x_{1}^{G}, \ldots, x_{n}^{G}\right) \leftrightarrow \exists p \in G\left(\bar{\varphi}\left(p, x_{1}, \ldots, x_{n}\right)\right)^{M} .
$$

O Lema da Verdade nos diz que qualquer fórmula verdadeira em $M[G]$ é forçada a ser verdadeira em $M[G]$, desde que alguma condição $p$, que vive em $M$, esteja em $G$. Isso quer dizer que conseguimos ter acesso a informações em $M[G]$ apenas olhando pra certas condições dentro de $M$. Essa fórmula $\bar{\varphi}\left(v, v_{1}, \ldots, v_{n}\right)$, que depende de $\varphi\left(v_{1}, \ldots, v_{n}\right)$ e de $\mathbb{P}$, é a relação de forcing que procuramos. Usaremos a seguinte notação: se $p \in \mathbb{P}, x_{1}, \ldots, x_{n} \in M$, denotaremos $\bar{\varphi}\left(p, x_{1}, \ldots, x_{n}\right)$ por $p \Vdash^{*} \varphi\left(x_{1}, \ldots, x_{n}\right)$. Agora, nossa tarefa é desvendar como deve ser essa relação $\Perp^{*}$.

Primeiro, é claro que devemos definir a fórmula $p \Vdash^{*} \varphi$ por recursão na complexidade de $\varphi$. Segundo, uma restrição importante é que se $p \Vdash^{*} \varphi$ e se $q$ é uma condição mais forte, então $q \Vdash^{*} \varphi$. Assim, a fim de que $p \Vdash^{*} \neg \psi$, não podemos ter alguma condição $q \leq p$ tal que $q \Vdash^{*} \varphi$. Com isso, obtemos trivialmente condições os casos $\vee, \exists$ e $\neg$. Antes de dar uma definição precisa da relação $\Vdash^{*}$, vamos investigar o caso atômico, sob a restrição do Lema da Verdade.

$$
\begin{aligned}
\exists p \in G\left(p \Vdash^{*} x \in y\right)^{M} & \leftrightarrow(x \in y)^{M[G]} \\
& \leftrightarrow x^{G} \in y^{G} \\
& \leftrightarrow \exists z\left(x \in_{G} y \wedge(z=x)^{M[G]}\right) \\
& \leftrightarrow \exists z \exists q \in G\left((x, q) \in y \wedge(z=x)^{M[G]}\right) \\
& \leftrightarrow \exists z \exists q\left((x, q) \in y \wedge q \in G \wedge(z=x)^{M[G]}\right) .
\end{aligned}
$$

Agora, note que, para validade do Lema da Verdade para fórmulas atômicas, $\exists q \in G(z=x)^{M[G]}$ deve ser equivalente a $\exists q \in G\left(q \Vdash^{*} z=y\right)^{M}$, o que sugere uma definição recursiva sobre o posto de $y$. Por outro lado, como na definição da relação de forcing em $M$ não podemos fazer referência a $G$, uma alternativa é trocar $\exists q \in G$ por $q \geq p$, como sendo uma condição mais fraca e impor que $p$ força $z=x$, para algum $z$. Assim, quando $p \in G$, isso implicará que $q \in G$.

Finalmente, o caso de fórmulas do tipo $x=y$ já foi mencionado. Sabemos que $x \neq y$ se, e somente se, existe $z \in x$ tal que $z \notin y$ ou se existe $z \in y$ tal que $z \notin x$. Isso soa estranhamente circular: o caso $x \in y$ recorre a fórmulas do tipo $z=w$, e o caso $x=y$ recorre a fórmulas do tipo $z \in w$. Isso é perfeitamente possível porque estamos definindo a relação $\mathbb{H}^{*}$ para os casos atômicos $x \in y$ e $x=y$ fazendo uso de recursão sobre os postos de $x$ e $y$.

Definição 3.1.15. Sejam $\mathbb{P} \in M$ uma noção de forcing, $p \in \mathbb{P}$ e $\varphi$ uma fórmula de $\mathcal{L}_{\in}$, definimos $p \Vdash^{*} \varphi$ por indução na complexidade da fórmula $\varphi$ e no posto dos elementos de $M$ :

- $p \Vdash^{*} x \in y \leftrightarrow \exists z \exists q \geq p\left((z, q) \in y \wedge p \Vdash^{*} z=x\right)$.

- $p \Vdash^{*} x \neq y \leftrightarrow \exists z \exists q \geq p\left((z, q) \in y \wedge p \Vdash^{*} z \notin x\right) \vee \exists z \exists q \geq p\left((z, q) \in x \wedge p \Vdash^{*} z \notin y\right)$;

- $p \Vdash^{*} \neg \varphi \leftrightarrow \forall q \leq p\left(q \nVdash^{*} \varphi\right)$.

- $p \Vdash^{*} \varphi \vee \psi \leftrightarrow p \Vdash^{*} \varphi \vee p \Vdash^{*} \psi$.

- $p \Vdash^{*} \exists x \varphi \leftrightarrow \exists x\left(p \Vdash^{*} \varphi(x)\right)$.

Com essa definição precisa de $\Vdash^{*}$ e alguma heurística acerca do Lema da Verdade, estamos prontos para de fato prová-lo. Mas antes, enunciaremos o seguinte lema, que decorre imediatamente da definição de $\Vdash^{*}$ :

Lema 3.1.16. Sejam $\mathbb{P}$ uma noção de forcing e $\varphi$ uma fórmula em $\mathcal{L}_{\in}$. 
1. Se $p \Vdash^{*} \varphi$ e $q \leq p$, então $q \Vdash^{*} \varphi$.

2. $p \Vdash^{*} \varphi \leftrightarrow \neg \exists q \leq p\left(q \Vdash^{*} \neg \varphi\right)$.

Teorema 3.1.17 (Lema da Verdade). Sejam $\mathbb{P} \in M$ uma noção de forcing e G um filtro $\mathbb{P}$ genérico sobre $M$. Então

$$
\varphi^{M[G]} \leftrightarrow \exists p \in G\left(p \Vdash^{*} \varphi\right)^{M} .
$$

Demonstração. Antes de fazer indução na complexidade da fórmula $\varphi$, vamos provar a seguinte:

Afirmação: Se o teorema é verdadeiro para uma fórmula $\varphi$, então

$$
x \in_{G} y \wedge \varphi^{M[G]} \leftrightarrow \exists p \in G \exists q \geq p\left((x, q) \in y \wedge\left(p \Vdash^{*} \varphi\right)^{M}\right)
$$

Demonstração.

- $(\Rightarrow)$ Como $x \in_{G} y$, existe $q \in G$ tal que $(q, x) \in y$. Como estamos assumindo o Lema da Verdade, se $\varphi^{M[G]}$ então existe $r \in G$ tal que $\left(r \Vdash^{*} \varphi\right)^{M}$. Assim, se $p \in G$ é tal que $p \leq q, r$, então $\left(p \Vdash^{*} \varphi\right)^{M}$.

- $(\Leftarrow)$ Sejam $p \in G$ e $q \geq p$ tais que $(x, q) \in y$ e $\left(p \Vdash^{*} \varphi\right)$. Primeiro, $q \in G$ e, portanto, $x \in_{G} y$. Segundo, como $\left(p \Vdash^{*} \varphi\right)^{M}$, pelo Lema da Verdade $\varphi^{M[G]}$.

Agora, faremos a demonstração desse teorema por indução na complexidade de $\varphi$. Os casos em que $\varphi$ é $\chi \vee \psi$, ou $\exists x \psi$, são triviais, e portanto consideraremos somente o caso atômico e a negação.

- $\varphi$ é $x \in y$.

Usaremos indução no posto de $y$ da seguinte forma: suponha que para todo $x \in y$, o Lema da Verdade valha para as fórmulas do tipo $z=x$. Isto é,

$$
(z=x)^{M[G]} \leftrightarrow \exists p \in G\left(p \Vdash^{*} z=x\right)^{M} .
$$

Já vimos que $(x \in y)^{M[G]}$ é equivalente a $\exists z\left(z \in_{G} y \wedge(z=x)^{M[G]}\right)$. Pela hipótese de indução no posto de $y$ e pela afirmação acima, isso é equivalente a

$$
\exists z \exists p \in G \exists q \geq p\left((x, q) \in y \wedge\left(p \Vdash^{*} z=x\right)^{M}\right)
$$

E trocando a ordem de $\exists z$ e $\exists p \in G$, concluímos que essa afirmação é equivalente a $\left(p \Vdash^{*} \varphi\right)^{M}$, usando algumas propriedades básicas de absolutividade e $\mathbb{P} \in M$, como queríamos.

- $\varphi$ é $x \neq y$.

A demonstração segue de forma análoga ao item anterior, usando a afirmação e indução sobre o posto de $y$ e sobre o posto de $x$, separando em dois casos.

- $\varphi$ é $\neg \psi$.

Antes de tudo, considere o conjunto

$$
D \doteq\left\{p \in \mathbb{P} \mid p \Vdash^{*} \psi \vee p \Vdash^{*} \neg \psi\right\} .
$$

Como $\mathbb{P} \in M$ e a relação de forcing é definível em $M$, já que é absoluta para modelos transitivos de ZFC, então $D \in M$. Além disso, se $p \in \mathbb{P}$, temos duas opções: ou $p \Vdash^{*} \neg \psi$, e portanto $p \in D$; ou não, e então por definição existe $q \leq p$ tal que $q \Vdash^{*} \psi$, e portanto $q \in D$, donde segue que $D$ é denso em $\mathbb{P}$. Assim, ou existe $p \in G$ tal que $p \Vdash^{*} \psi$, ou existe $p \in G$ 
tal que $p \Vdash^{*} \neg \psi$, e os dois casos são mutuamente exclusivos (é fácil ver, pois $G$ é um filtro). Assim, usando essa observação e o Lema da Verdade para $\psi$ :

$$
\begin{aligned}
(\neg \psi)^{M[G]} & \leftrightarrow \neg \psi^{M[G]} \\
& \leftrightarrow \neg\left(\exists p \in G\left(p \Vdash^{*} \psi\right)^{M}\right) \\
& \leftrightarrow \exists p \in G\left(p \Vdash^{*} \neg \psi\right)^{M} .
\end{aligned}
$$

Teorema 3.1.18 (Teorema Fundamental do Forcing). Sejam $\mathbb{P} \in M$ uma noção de forcing e $\varphi$ uma $\mathcal{L}_{\in}$-fórmula. Então

$$
\left(p \Vdash^{*} \varphi\right)^{M} \leftrightarrow p \Vdash \varphi .
$$

Demonstração. Suponha $\left(p \Vdash^{*} \varphi\right)^{M}$. Seja $G$ um filtro $\mathbb{P}$-genérico qualquer tal que $p \in G$. Pelo Lema da Verdade (Teorema 3.1.17) temos $\varphi^{M[G]}$.

Para a recíproca, suponha $p \Vdash \varphi$ e $\left(p \nVdash^{*} \varphi\right)^{M}$. Então pelo item 2 do Lema 3.1.16 deve existir $q \leq p$ tal que $\left(q \Vdash^{*} \neg \varphi\right)^{M}$, o que por definição significa que não pode existir $s \leq q$ tal que $s \Vdash^{*} \varphi$. Seja $G$ um filtro genérico tal que $q \in G$. Como $q \leq p$, temos $p \in G$, e então $\varphi^{M[G]}$, por hipótese. Pelo Lema da Verdade, deve existir $r \in G$ tal que $\left(r \Vdash^{*} \varphi\right)^{M}$. Agora, como $p, r \in G$ e $G$ é filtro, seja $s$ uma extensão de $p$ e $q$. Então $\left(s \Vdash^{*} \varphi\right)^{M}$, o que é absurdo.

Definição 3.1.19. Seja $\mathbb{P}$ uma noção de forcing. Definiremos nomes canônicos para elementos de $M$ e um nome canônico para um $\mathbb{P}$-genérico sobre $M$.

1. O nome canônico para um elemento de $M$ é definido por $\check{x} \doteq\{(\check{y}, \mathbb{1}) \mid y \in x\}$.

2. O nome canônico para um filtro $\mathbb{P}$-genérico sobre $M$ é definido por $\dot{G} \doteq\{(\check{p}, p) \mid p \in \mathbb{P}\}$.

Teorema 3.1.20. Sejam $\mathbb{P}$ uma noção de forcing e $G$ um filtro $\mathbb{P}$-genérico sobre $M$.

1. $M \subseteq M[G]$.

2. $G \in M[G]$.

3. $M \cap \mathrm{ON}=M[G] \cap \mathrm{ON}$.

4. Se $N$ é um modelo transitivo enumerável de ZFC tal que $M \subseteq N$ e $G \in N$, então $M[G] \subseteq N$ - isto é, $M[G]$ é o menor modelo transitivo enumerável satisfazendo 1. e 2.

Demonstração.

1. Seja $x \in M$. Basta provar por indução no posto de $x$ que $\check{x}^{G}=x$ : de fato, suponha que $\check{y}^{G}=y$, para todo $y \in x$. Então

$$
\check{x}^{G}=\left\{\check{y}^{G} \mid y \in x\right\}=\{y \mid y \in x\}=x .
$$

2. Basta notar que $\dot{G}^{G}=G$. De fato,

$$
\dot{G}^{G}=\left\{\check{p}^{G} \mid p \in G\right\}=\{p \mid p \in G\}=G .
$$

3. É fácil mostrar por indução que $\rho\left(x^{G}\right) \leq \rho(x)$ para qualquer $x \in M$. Sejam então $\alpha \in$ $M[G] \cap \mathrm{ON}$ e $\dot{\alpha}$ um $\mathbb{P}$-nome para $\alpha$. Temos que $\alpha=\rho(\alpha) \leq \rho(\dot{\alpha}) \in M \cap \mathrm{ON}$, pela absolutividade da função posto. Assim, $M[G] \cap \mathrm{ON} \subseteq M \cap \mathrm{ON}$ e $M[G] \cap \mathrm{ON}=M \cap \mathrm{ON}$ segue de $M \subseteq M[G]$. 
4. Seja $x \in M$ e considere $x^{G} \in M[G]$. Como a definição do colapso $\pi_{M, G}$ é absoluta para modelos transitivos de ZFC e $x, G \in N$, então $x^{G} \in N$.

Lema 3.1.21. Sejam $\mathbb{P} \in M$ uma noção de forcing e $x, y \in M$.

1. Se $\operatorname{par}(x, y) \doteq\{(x, \mathbb{1}),(y, \mathbb{1})\}$, então $(\operatorname{par}(x, y))^{G}=\left\{x^{G}, y^{G}\right\}$, i.e., $\operatorname{par}(x, y)$ é um $\mathbb{P}$-nome para o par não ordenado $\left\{x^{G}, y^{G}\right\}$.

2. Se $\operatorname{po}(x, y) \doteq \operatorname{par}(\operatorname{par}(x, x), \operatorname{par}(x, y))$, então $(\operatorname{po}(x, y))^{G}=\left(x^{G}, y^{G}\right)$, i.e., $\operatorname{po}(x, y)$ é um $\mathbb{P}$ nome para o par ordenado $\left(x^{G}, y^{G}\right)$.

Teorema 3.1.22. Sejam $\mathbb{P} \in M$ uma noção de forcing e $G$ um filtro $\mathbb{P}$-genérico sobre $M$. Então

$$
M[G] \models \mathrm{ZFC} .
$$

Demonstração. É fácil verificar em $M[G]$ os Axioma da Extensionalidade, da Regularidade e do Infinito. É um resultado simples verificar que o Axioma da Separação segue do Axioma da Substituição. O Axioma do Par segue do Lema 3.1.21. Assim, verificaremos o Axioma da União, o Axioma das Partes, o Axioma da Substituição e o Axioma da Escolha. Seguimos a enumeração da Definição 1.3.1.

\section{Axioma da União.}

Seja $x \in M[G]$ e considere $\dot{x}$ um $\mathbb{P}$-nome para $x$. Basta verificar que o conjunto

$$
\dot{y} \doteq\{(\dot{w}, p) \mid \exists(\dot{s}, q) \in \dot{x} \exists r((\dot{w}, p) \in \dot{s} \wedge p \leq r \wedge p \leq q)\}
$$

é um $\mathbb{P}$-nome e $y \doteq \dot{y}^{G}=\bigcup x$.

\section{Axioma das Partes.}

Seja $x \in M[G]$ e considere $\dot{x}$ um $\mathbb{P}$-nome para $x$. A ideia é encontrar um conjunto grande $Q \in M$ que tenha nomes para todos os subconjuntos de $x$ em $M[G]$. Assim, sejam

$$
Q \doteq(\mathcal{P}(\operatorname{dom}(\dot{x}) \times \mathbb{P}))^{M}, \dot{y} \doteq Q \times\{\mathbb{1}\} \text { e } y \doteq \dot{y}^{G} .
$$

Vamos verificar que $(\mathcal{P}(x))^{M[G]}=\mathcal{P}(x) \cap M[G] \subseteq y$. Assim, sejam $z \in \mathcal{P}(x) \cap M[G], \dot{z} \in M$ tal que $\dot{z}^{G}=z$ e considere $\dot{w} \doteq\{(\dot{s}, p) \mid \dot{s} \in \operatorname{dom}(\dot{x}) \wedge p \Vdash \dot{s} \in \dot{z}\}$. Como a relação de forcing é definível em $M$, então $\dot{w} \in M$. Além disso, $\dot{w} \in Q$, então só precisamos provar que $\dot{w}^{G}=z$. De fato, $\dot{w}^{G} \subseteq z$ pois $\dot{w}^{G}=\left\{\dot{s}^{G} \mid \exists p \in G(p \Vdash \dot{s} \in \dot{z})\right\}$ e todos os $\dot{s}^{G}$ estão em $\dot{z}^{G}=z$ pelo Teorema 3.1.18. Para a outra inclusão, como $z \subseteq x=\dot{x}^{G}$, pelo Lema da Verdade (Teorema 3.1.17), existe $p \in G$ tal que $p \Vdash \dot{s} \in \dot{z}$, e então $(\dot{s}, p) \in \dot{w}, \operatorname{logo} \dot{s}^{G} \in \dot{w}^{G}$.

\section{Axioma da Substituição.}

Seja $\varphi$ uma $\mathcal{L}_{\epsilon}$-fórmula em que $Z$ não ocorre livre e seja $x \in M[G]$. Assuma

$$
(\forall y \in x \exists z \varphi(y, z))^{M[G]} .
$$

Vamos mostrar que existe $Z \in M[G]$ tal que $(\forall y \in x \exists z \in Z \varphi(y, z))^{M[G]}$

Seja $\dot{x}$ um $\mathbb{P}$-nome para $x$. Trabalhando em $M$, usando a definibilidade da relação de forcing, seja $Q$ um conjunto de nomes com a seguinte propriedade: para todo $p \in \mathbb{P}$ e todo $\dot{y} \in \operatorname{dom}(\dot{x})$, se existe $\dot{z}$ tal que $p \Vdash \varphi(\dot{y}, \dot{z})$, então pelo menos um $\operatorname{dos} \dot{z}$ está em $Q$. Sejam $\dot{Z} \doteq Q \times\{\mathbb{1}\}$ e $Z \doteq \dot{Z}^{G}$. Agora sejam $y \in x$ e $\dot{y} \in \operatorname{dom}(\dot{x})$ um $\mathbb{P}$-nome para $y$. Como $(\exists z \varphi(y, z))^{M[G]}$, então existe $\dot{z}$ um $\mathbb{P}$-nome tal que $\left(\varphi\left(y, \dot{z}^{G}\right)\right)^{M[G]}$, e pelo Lema da Verdade, existe $p \in G$ tal que $p \Vdash \varphi(\dot{y}, \dot{z})$. Então pela propriedade do $Q$, podemos assumir que $\dot{z} \in Q$. Se $z \doteq \dot{z}^{G}$, então $z \in Z$ e $(\varphi(y, z))^{M[G]}$ 


\section{Axioma da Escolha.}

Provaremos que todo conjunto em $M[G]$ possui uma boa-ordem em $M[G]$. Assim, sejam $x \in M[G]$ e $\dot{x}$ um $\mathbb{P}$-nome para $x$. Em $M$, use AC para bem-ordenar $\operatorname{dom}(\dot{x})=\left\{\dot{y}_{\xi} \mid \xi<\alpha\right\}$, e seja $\dot{f} \doteq\left\{\left(\operatorname{op}\left(\check{\xi}, \dot{y}_{\xi}\right), \mathbb{1}\right) \mid \xi<\alpha\right\}$. Agora seja $f \doteq \dot{f}^{G}$. Pelo Lema 3.1.21, $f=\left\{\left(\xi, \dot{y}_{\xi}^{G}\right) \mid \xi<\alpha\right\}$, e então $f$ é uma função tal que $\operatorname{dom}(f)=\alpha$ e $x \subseteq \operatorname{im}(f)$. Então, trabalhando em $M[G]$, podemos bem-ordenar $x$ por $\triangleleft$ definindo:

$$
a \triangleleft b \leftrightarrow \min \{\xi<\alpha \mid f(\xi)=a\}<\min \{\xi<\alpha \mid f(\xi)=b\} .
$$

Encerramos essa seção fazendo a seguinte convenção: ao invés de usar a linguagem $\mathcal{L}_{\in}(M)$ e a teoria $\mathrm{ZFC}_{M}$, pensaremos que estamos trabalhando na teoria ZFC, e usaremos V "como modelo". $\mathrm{V}$ trivialmente tem a propriedade de "ser transitivo". Além disso, quando trabalhamos em $\mathrm{ZFC}_{M}$, todas as noções envolvidas no nosso argumento de forcing são relativizadas para $M$, e tomamos o cuidado de verificar que as noções relevantes são absolutas para modelos transitivos de ZFC (para que possamos transitar entre os modelos). Com isso, outro cuidado que precisamos tomar é sempre assegurar que as noções envolvidas são absolutas para modelos transitivos de ZFC. Finalmente, o papel da enumerabilidade é exclusivamente usado para construir um filtro genérico $G$ fora de $M$. Podemos pensar que existe um filtro $G$ "fora" de V. Dito isso, fica claro que essa convenção é simplesmente notacional, sendo sempre possível adaptar os argumentos de forcing para a teoria $\mathrm{ZFC}_{M}$ e usar os teoremas metamatemáticos 3.1.6 e 3.1.7. Essa convenção segue a literatura usual de forcing, e sua necessidade ficará clara na Seção ??. Além disso, também diremos apenas " $G$ é filtro $\mathbb{P}$-genérico" e não " $G$ é filtro $\mathbb{P}$-genérico sobre V".

\subsection{Tecnologia do forcing}

Na seção anterior ficou claro que assumimos que o filtro genérico vive "fora" de V (que antes era $M)$. Mas pode não ser o caso em que, para algumas noções de forcing, o filtro está em $V$. Nesse caso, a extensão genérica é dita trivial (é o caso em que $\mathrm{V}=\mathrm{V}[G]$ ). Não que extensões genéricas triviais sejam de todo desinteressantes, às vezes, inclusive, têm uma ou outra aplicação. Consideraremos apenas extensões genéricas não triviais, e veremos agora uma condição suficiente para que uma dada extensão genérica não seja trivial.

Definição 3.2.1. Seja $\mathbb{P}$ uma noção de forcing. Dizemos que $r \in \mathbb{P}$ é um átomo se não existem $p, q \leq r \operatorname{com} p \perp q$. Se $\mathbb{P}$ não possui átomos, então $\mathbb{P}$ é dito forcing não-atômico.

Teorema 3.2.2. Sejam $\mathbb{P}$ um forcing não-atômico e $G$ um filtro $\mathbb{P}$-genérico. Então $G \notin \mathrm{V}$.

Demonstração. Suponha que $G \in \mathrm{V}$ e considere $D=\mathbb{P} \backslash G$. É fácil ver que $D$ é um denso tal que $D \in V$, pois $\mathbb{P}, G \in V$ e "\" tem definição absoluta. Segue que $D \cap G \neq \emptyset$, absurdo.

Para $\kappa>\omega$ um cardinal, considere $\mathbb{P}=\operatorname{Fn}(\omega, \kappa)$ o forcing das funções finitas com domínio contido em $\omega$ e imagem em $\kappa$, que já foi considerado na seção anterior. Pelo que vimos, se $G$ é um filtro $\mathbb{P}$-genérico, então $f \doteq \bigcup G$ é uma função $f: \omega \rightarrow \kappa$ sobrejetora, e então $\kappa$ é enumerável em $\mathrm{V}[G]$. Embora isso soe contraditório, isso não diz além de "cardinalidade não é uma noção absoluta para modelos transitivos de ZFC". No nosso exemplo, o cardinal $\kappa$ deixou de ser um cardinal, mesmo ainda sendo um ordinal enumerável em $\mathrm{V}[G]$ (lembre que $\omega$ é absoluto, então $\kappa$ não poderia ser $\omega$, já que é não-enumerável). Cohen já havia percebido esse problema do colapso de cardinais enquanto tentava provar a consistência de $\neg \mathrm{CH}$ :

"[...]Entretanto, há uma complicação. A saber, o $\aleph_{2}$ que estamos usando é aquele ordinal do modelo original $M$. Mas o enunciado de $\mathrm{CH}$ é que a cardinalidade do continuum é o primeiro cardinal não enumerável. É necessário então provar que $\aleph_{2}$ em $M$ é o segundo cardinal não enumerável no novo modelo." $\quad\left[\mathrm{C}^{+} 02\right]$ 
Definição 3.2.3. Sejam $\mathbb{P}$ uma noção de forcing e $\kappa$ um cardinal.

1. Dizemos que $\mathbb{P}$ preserva cardinais acima de $\kappa$ se para todo cardinal $\theta \geq \kappa$,

$$
\Vdash \check{\theta} \text { é um cardinal. }
$$

2. Dizemos que $\mathbb{P}$ preserva cofinalidades acima de $\kappa$ se para todo ordinal limite $\gamma$ tal que $\operatorname{cf}(\gamma) \geq \kappa$,

$$
\Vdash \operatorname{cf}(\check{\gamma})=\operatorname{cf} \check{(} \gamma)
$$

Note que $\Vdash \operatorname{cf}(\check{\gamma}) \leq \operatorname{cf} \check{(}(\gamma)$, pela definição de cofinalidade e argumentos de absolutividade.

Lema 3.2.4. Sejam $\mathbb{P}$ uma noção de forcing e $\kappa$ um cardinal regular.

1. $\mathbb{P}$ preserva cofinalidades acima de $\kappa$ se, e somente se, para todo cardinal regular $\theta \geq \kappa$,

$$
\Vdash \check{\theta} \text { é regular. }
$$

2. Se $\mathbb{P}$ preserva cofinalidades acima de $\kappa$, então $\mathbb{P}$ preserva cardinais acima de $\kappa$.

Demonstração.

1. Um dos lados é trivial: se $\mathbb{P}$ preserva cofinalidades, então $\mathbb{P}$ preserva os cardinais regulares. Para a recíproca, considere $\gamma$ ordinal limite tal que $\operatorname{cf}(\gamma) \geq \kappa$ e seja $\theta \doteq \operatorname{cf}(\gamma)$. Assim, existe uma função $f: \theta \rightarrow \gamma$ estritamente crescente e cofinal. Como $\theta$ é regular, então $\left(\theta\right.$ é regular) ${ }^{\mathrm{V}[G]}$, por hipótese. Como $f \in \mathrm{V}[G]$, então $\theta=\mathrm{cf}^{\mathrm{V}[G]}(\gamma)$.

2. Todo cardinal ou é regular, ou é $\leq \omega$, ou é supremo de cardinais regulares sucessores. O primeiro caso é o item 1., o segundo caso segue por absolutividade e o terceiro caso segue por absolutividade para o supremo e pelo item 1 .

A relação entre as cardinalidades para anticadeias e preservação de cardinais também foi percebida por Cohen enquanto argumentava para provar a Hipótese do Continuum.

"[...]É possível mostrar que se dois ordinais têm cardinalidades diferentes em $M$, então eles têm cardinalidades diferentes no novo modelo. Existe um fato importante sobre condições elementares que é responsável por isso, a condição de cadeia enumerável"

$$
\left[\mathrm{C}^{+} 02\right]
$$

Definição 3.2.5. Sejam $\mathbb{P}$ uma noção de forcing $e A \subseteq \mathbb{P}$. Dizemos que $A$ é anticadeia em $\mathbb{P}$ se para quaisquer $p, q \in A, p \perp q$.

Definição 3.2.6. Sejam $\mathbb{P}$ uma noção de forcing e $\kappa$ um cardinal. Dizemos que $\mathbb{P}$ tem $\kappa$-c.c. ( $\kappa$ chain-condition) se toda anticadeia em $\mathbb{P}$ tem cardinalidade menor do que $\kappa$. Se $\mathbb{P}$ tem $\omega_{1}$-c.c., então dizemos que $\mathbb{P}$ tem c.c.c. (countable chain condition).

Provaremos que forcings que têm $\kappa-$ c.c. preservam cardinais acima de $\kappa$. Antes disso, precisamos de um lema bastante útil e de uma definição que já apareceu implicitamente na seção anterior:

Definição 3.2.7. Sejam $\mathbb{P}$ uma noção de forcing, $p \in \mathbb{P} e D \subseteq \mathbb{P}$.

1. $\overleftarrow{p} \doteq\{q \in \mathbb{P} \mid q \leq p\}$

2. Dizemos que $D$ é denso abaixo de $p \in \mathbb{P}$ se $D \cap \overleftarrow{p}$ é denso em $\overleftarrow{p}$ 
Lema 3.2.8. Sejam $\mathbb{P}$ uma noção de forcing, $p \in \mathbb{P}$ e $\varphi$ uma $\mathcal{L}_{\in}$-fórmula. Então $p \Vdash \varphi$ se, e somente se, $\{q \leq p \mid q \Vdash \varphi\}$ é denso abaixo de $p$.

Teorema 3.2.9. Sejam $\mathbb{P}$ uma noção de forcing e $\kappa$ um cardinal regular. Se $\mathbb{P}$ tem $\kappa$-c.c., então $\mathbb{P}$ preserva cofinalidades acima de $\kappa$, e portanto preserva cardinais acima de $\kappa$.

Demonstração. Pelo Lema 3.2.4 é suficiente mostrar que $\mathbb{P}$ preserva os cardinais regulares acima de $\kappa$. Seja então $\theta \geq \kappa$ um cardinal regular e $\lambda<\theta$, vamos mostrar que toda função $f \in \mathrm{V}[G]$, $f: \lambda \rightarrow \theta$ é limitada (e portanto não pode ser cofinal em $\theta$ ). Seja $\dot{f}$ um $\mathbb{P}$-nome para $f$ e pelo Lema da Verdade seja $p \in G$ tal que

$$
p \Vdash \dot{f}: \check{\lambda} \rightarrow \check{\theta} .
$$

Agora vamos considerar condições que decidem os valores de $\dot{f}(\alpha)$, para cada $\alpha<\lambda$ :

$$
A_{\alpha} \doteq\{\beta<\theta \mid \exists q \leq p(q \Vdash \dot{f}(\check{\alpha})=\check{\beta})\} .
$$

Note que $\left|A_{\alpha}\right|<\kappa$, para todo $\alpha<\lambda$. De fato, fixe $\alpha<\lambda$ e seja, para cada $\beta<\theta, W_{\alpha}^{\beta} \doteq\{q \leq$ $p \mid q \Vdash \dot{f}(\check{\alpha})=\breve{\beta}\}$ o conjunto das condições que forçam $f(\alpha)=\beta$. Podemos escolher para uma única condição $q_{\beta} \in W_{\alpha}^{\beta}$ usando uma função de escolha $h_{\alpha}:\left\{W_{\alpha}^{\beta}\right\}_{\beta<\theta} \rightarrow \bigcup_{\beta<\theta} W_{\alpha}^{\beta}$. Assim $W_{\alpha} \doteq \operatorname{im}\left(h_{\alpha}\right)$ é trivialmente uma anticadeia, e portanto tem cardinalidade $<\kappa$. O mapa $\beta \mapsto q_{\beta}$ que escolhe para cada $\beta \in A_{\alpha}$, algum $q_{\beta} \in W_{\alpha}$, é uma injeção, e portanto $\left|A_{\alpha}\right|<\kappa$.

Agora, como $\theta$ é regular, então $\left|\bigcup_{\alpha<\theta} A_{\alpha}\right|<\theta$ e, portanto, $\bigcup_{\alpha<\theta} A_{\alpha}$ é limitado por algum $\gamma<\theta$. Segue que para cada $\alpha<\lambda, p \Vdash \dot{f}(\check{\alpha})<\check{\gamma}$, pois pelo que argumentamos $\{q \leq p \mid q \Vdash \dot{f}(\check{\alpha})<\check{\gamma}\}$ é denso abaixo de $p$. Pelo Teorema Fundamental do Forcing, segue que toda função $f: \lambda \rightarrow \theta$ de $\mathrm{V}[G]$ é limitada em $\mathrm{V}[G]$ e, portanto, $(\theta \text { é regular })^{\mathrm{V}[G]}$.

Para nossos propósitos, também é importante analisar a preservação de cardinais abaixo de algum cardinal. $\mathrm{O}$ caso em que estaremos interessados é quando esse cardinal é $\aleph_{1}$.

Definição 3.2.10. Sejam $\mathbb{P}$ uma noção de forcing e $\lambda$ um cardinal.

1. Dizemos que $\mathbb{P}$ preserva cardinais abaixo de $\kappa$ se para todo cardinal $\theta \leq \kappa$,

$\Vdash \check{\theta}$ é um cardinal.

2. Dizemos que $\mathbb{P}$ preserva cofinalidades abaixo de $\kappa$ se para todo ordinal limite $\gamma$ tal que $\operatorname{cf}(\gamma) \leq \kappa$,

$$
\Vdash \operatorname{cf}(\check{\gamma})=\operatorname{cf} \check{(}(\gamma)
$$

Lema 3.2.11. Sejam $\lambda$ um cardinal, $\mathbb{P}$ um forcing $\lambda$-fechado e $\delta<\lambda$ tais que $\Vdash \forall f \in \check{\lambda}^{\check{\delta}}(f \in \check{V})$. Então $\mathbb{P}$ preserva cofinalidades, e portanto cardinais abaixo de $\kappa$.

Definição 3.2.12. Sejam $\mathbb{P}$ uma noção de forcing e $\lambda$ um cardinal. Dizemos que $\mathbb{P}$ é um forcing $\lambda$-fechado se para todo $\delta<\lambda$ e toda $\delta$-sequência não-crescente $\left(p_{\gamma}\right)_{\gamma<\delta}$ de condições em $\mathbb{P}$, existe $p \in \mathbb{P}$ tal que $p \leq p_{\gamma}$ para qualquer $\gamma<\delta$. Se $\mathbb{P}$ é $\omega_{1}$-fechado, então dizemos que $\mathbb{P}$ é $\sigma$-fechado. $\dashv$

Teorema 3.2.13. Sejam $\lambda$ um cardinal, $\mathbb{P}$ um forcing $\lambda$-fechado e $\delta<\lambda$. Então

$$
\Vdash \forall f \in \check{\lambda} \check{\delta}(f \in \check{V}) .
$$

Demonstração. Sejam $G$ um filtro $\mathbb{P}$-genérico e $f \in \mathrm{V}[G] \operatorname{com} f: \delta \rightarrow E$, para algum conjunto $E$. Considere $p \in G$ tal que

$$
p \Vdash \dot{f}: \check{\delta} \rightarrow \check{E} .
$$

Vamos provar que existe uma função $h \in V$ e existe $q \leq p, q \Vdash \dot{f}=\check{h}$. Definiremos uma $(\delta+1)$ sequência não-crescente de elementos de $\mathbb{P}$ recursivamente da seguinte forma: 
- $p_{0}=p$;

- escolhemos $p_{\beta+1} \leq p_{\beta}$ e $e_{\beta}$ tais que $p_{\beta+1} \Vdash \dot{f}(\check{\beta})=\check{e}_{\beta}$;

- se $\gamma \leq \delta$ é limite, escolhemos $p_{\gamma}$ tal que $p_{\gamma} \leq p_{\beta}$, para todo $\beta<\gamma$; isso é possível pois $\mathbb{P}$ é $\lambda$-fechado.

Defina $h(\beta)=e_{\beta}$ e tome $q=p_{\delta}$. Então $q \Vdash \dot{f}(\check{\beta})=\check{h}(\check{\beta})$, para todo $\beta<\delta$ e, portanto, $q \Vdash \dot{f}=\check{h}$.

Corolário 3.2.14. Sejam $\mathbb{P}$ uma noção de forcing e $\lambda$ um cardinal. Se $\mathbb{P}$ é $\lambda$-fechado, então $\mathbb{P}$ preserva cofinalidades abaixo de $\lambda$, e portanto preserva cardinais abaixo de $\lambda$.

Agora introduziremos algumas definições usuais da literatura de forcing e provaremos algumas equivalências sobre genericidade.

Definição 3.2.15. Sejam $\mathbb{P}$ é uma noção de forcing e $D \subseteq \mathbb{P}$. Dizemos que $D$ é predenso em $\mathbb{P}$ se para todo $p \in \mathbb{P}$, existe $q \in D$ tal que $p \| q$. Equivalentemente, $\neg \exists p(p \perp D)$.

Definição 3.2.16. Sejam $\mathbb{P}$ é uma noção de forcing e $E \subseteq \mathbb{P}$.

1. A topologia da ordem em $\mathbb{P}$ é definida por $\tau_{\mathbb{P}} \doteq\{O \subseteq \mathbb{P} \mid \forall p \in O(\overleftarrow{p} \subseteq O)\}$

2. $\overleftarrow{E} \doteq \bigcup\{\overleftarrow{p} \mid p \in E\}$ é o menor aberto (com a topologia da ordem, acima) que contém E. $\quad$

O Teorema 3.2.19 é uma equivalência para genericidade extremamente útil para inúmeros argumentos de forcing.

Lema 3.2.17. Sejam $\mathbb{P}$ uma noção de forcing e $E \subseteq \mathbb{P}$. São equivalentes:

1. E é predenso.

2. $\overleftarrow{E}$ é denso.

3. $\overleftarrow{E}$ é aberto denso.

Lema 3.2.18. Sejam $\mathbb{P}$ uma noção de forcing e $E \subseteq \mathbb{P}$. Então $G \cap E \neq \emptyset$ se, e somente se, $G \cap \overleftarrow{E} \neq \emptyset$

Teorema 3.2.19. Sejam $\mathbb{P}$ uma noção de forcing e $G \subseteq \mathbb{P}$ um filtro. São equivalentes:

1. Gé $\mathbb{P}$-genérico.

2. $G \cap E \neq \emptyset$, sempre que $E$ é predenso.

3. $G \cap E \neq \emptyset$, sempre que $E$ é anticadeia maximal.

4. $G \cap E \neq \emptyset$, sempre que $E$ é aberto denso.

Demonstração.

- $(1 . \Rightarrow 2$.) Se $E$ é predenso, então $\overleftarrow{E}$ é denso, pelo Lema 3.2.17. Assim, $G \cap \overleftarrow{E} \neq \emptyset$ e então $G \cap E \neq \emptyset$, pelo Lema 3.2.18.

- $(2 . \Rightarrow 3$.) Basta observar que se $E$ é uma anticadeia maximal, então $E$ também é predenso pois, caso contrário, teríamos que existe $p \in \mathbb{P}$ tal que $p \perp E$, e então $E^{\prime}=E \cup\{p\}$ seria uma anticadeia contendo $E$ propriamente, o que é absurdo. 
- (3. $\Rightarrow 4$.) Basta observar que se $E$ é denso, então $E$ contém uma anticadeia que é maximal. De fato, considere $A \subseteq E$ uma anticadeia e suponha que não seja maximal em $\mathbb{P}$. Seja então $A^{\prime}=A \cup\{p\}$ tal que $p \perp A$. Como $E$ é denso, então existe $q \leq p$ tal que $q \in E$ e, portanto, $q \perp A$. Logo, $A \cup\{q\}$ não é maximal em $E$. Assim, como existe uma anticadeia maximal contida em $E$, esta cadeia deve ser também maximal em $\mathbb{P}$.

- $(4 . \Rightarrow 1$.) Basta observar que se $E$ é denso, então $\overleftarrow{E}$ é aberto denso e usar o Lema 3.2.18.

A noção de equivalência-equivalência surge quando nos fazemos a seguinte pergunta bastante natural:

Para duas noções de forcing $\mathbb{P}$ e $\mathbb{Q}$, o que significa dizer que elas produzem as mesmas extensões genéricas?

Definição 3.2.20. Sejam $\left(\mathbb{P}, \leq_{\mathbb{P}}, \mathbb{1}_{\mathbb{P}}\right)$ e $\left(\mathbb{Q}, \leq_{\mathbb{Q}}, \mathbb{1}_{\mathbb{Q}}\right)$ noções de forcing. Dizemos que $\mathbb{P}$ e $\mathbb{Q}$ são forcing-equivalentes, ou simplesmente equivalentes, se para todo filtro $G \mathbb{P}$-genérico, existe $H$ um filtro $\mathbb{Q}$-genérico tal que $\mathrm{V}[G]=\mathrm{V}[H]$, e reciprocamente, para todo $H$ filtro $\mathbb{Q}$-genérico existe $G$ filtro $\mathbb{P}$-genérico tal que $\mathrm{V}[G]=\mathrm{V}[H]$.

As condições suficientes para que duas noções de forcing sejam equivalentes são obtidas explorando mergulhos de uma noção de forcing em outra. Esses mergulhos devem ser funções com propriedades que envolvem todas os elementos importantes relacionados à técnica do forcing. Por exemplo, além de ser um isomorfismo de ordem, devem preservar incompatibilidade. Além desses requerimentos, mergulhos de particular interesse serão os mergulhos que preservam anticadeias maximais, ou mergulhos que criam cópias densas.

Definição 3.2.21. Sejam $\left(\mathbb{P}, \leq_{\mathbb{P}}, \mathbb{1}_{\mathbb{P}}\right),\left(\mathbb{Q}, \leq_{\mathbb{Q}}, \mathbb{1}_{\mathbb{Q}}\right)$ noções de forcing e $i: \mathbb{P} \rightarrow \mathbb{Q}$ com as sequintes propriedades:

- $i\left(\mathbb{1}_{\mathbb{P}}\right)=\mathbb{1}_{\mathbb{Q}}$.

- $\forall p_{0}, p_{1} \in \mathbb{P}\left(p_{0} \leq_{\mathbb{P}} p_{1} \rightarrow i\left(p_{0}\right) \leq_{\mathbb{Q}} i\left(p_{1}\right)\right)$.

- $\forall p_{0}, p_{1} \in \mathbb{P}\left(p_{0} \perp_{\mathbb{P}} p_{1} \leftrightarrow i\left(p_{0}\right) \perp_{\mathbb{Q}} i\left(p_{1}\right)\right)$.

Dizemos que $i$ é

1. mergulho completo se para qualquer $A \subseteq \mathbb{P}$ anticadeia maximal em $\mathbb{P}, i(A)$ é anticadeia maximal em $\mathbb{Q}$.

2. mergulho denso se $i$ é um mergulho completo e $i(\mathbb{P})$ é denso em $\mathbb{Q}$.

A existência de mergulhos densos entre noções de forcing é suficiente para provar que elas são equivalentes. Além disso, a relação de forcing em cada uma das noções de forcing pode ser transportada por esse mergulho denso. Esses comentários estão resumidos no Teorema 3.2.25, e os próximos lemas auxiliam em sua demonstração. Antes de tudo, vamos introduzir a transcrição dos nomes via mergulhos:

Lema 3.2.22. Sejam $\mathbb{P}$ e $\mathbb{Q}$ noções de forcing e $i: \mathbb{P} \rightarrow \mathbb{Q}$ um mergulho completo e $H \subseteq \mathbb{Q}$ um filtro $\mathbb{Q}$-genérico. Então $G \doteq i^{-1}(H)$ é $\mathbb{P}$-genérico e $\mathrm{V}[G] \subseteq \mathrm{V}[H]$

Demonstração. É fácil verificar que $G$ é filtro, usando a definição de mergulho completo. Para mostrar que $G$ é $\mathbb{P}$-genérico, pelo Teorema 3.2.19 é suficiente mostrar que $G \cap A \neq \emptyset$ sempre que $A$ é uma anticadeia maximal em $\mathbb{P}$. Para isso, basta observar que $i(A)$ é uma anticadeia maximal em $\mathbb{Q}$, pois $i$ é mergulho completo e, portanto, $G \cap i(A) \neq \emptyset$. Logo, $G \cap A \neq \emptyset$.

Agora, tome $x \in \mathrm{V}[G]$ e seja $\dot{x}$ um $\mathbb{P}$-nome para $x$. Para provar que $\dot{x}^{G} \in \mathrm{V}[H]$, precisamos achar um $\mathbb{Q}$-nome $\dot{y}$ tal que $x=\dot{y}^{H}$. Usando $i$ podemos induzir para o nome $\dot{x}$ um $\mathbb{Q}$-nome $i_{*}(\dot{x})$ recursivamente: $\left.i_{*}(\dot{x})=\left\{\left(i_{*}(\dot{z}), i(q)\right)\right) \mid(\dot{z}, q) \in \dot{x}\right\}$. É fácil checar que $i_{*}(\dot{x})$ é um $\mathbb{Q}$-nome tal que $i_{*}(\dot{x})^{H}=\dot{x}^{G}=x$. Logo, $x \in \mathrm{V}[H]$. 
A demonstração anterior sugere a necessidade de transcrever $\mathbb{P}$-nomes para $\mathbb{Q}$-nomes, usando o mapa $i$, e por isso, introduzimos a seguinte definição:

Definição 3.2.23. Sejam $\mathbb{P}$ e $\mathbb{Q}$ noções de forcing, e $i: \mathbb{P} \rightarrow \mathbb{Q}$. Para cada $x$, definimos o $\mathbb{Q}$-nome $i_{*}(x)$, a transcrição de $x$ via $i$, como sendo $i_{*}(x)=\left\{\left(i_{*}(y), i(q)\right) \mid(y, q) \in x\right\}$.

Lema 3.2.24. Sejam $\mathbb{P}$ uma noção de forcing e $G_{1}, G_{2}$ filtros $\mathbb{P}$-genéricos. Se $G_{1} \subseteq G_{2}$, então $G_{1}=G_{2}$.

Demonstração. Fixe $p \in G_{2}$ e considere $D \doteq\{q \in \mathbb{P} \mid q \leq p \vee q \perp p\}$. D é denso, então existe $q \in G_{1} \cap D \subseteq G_{2} \cap D$. Assim, ou $q \leq p$ ou $q \perp p$. O último não pode ocorrer pois $q, p \in G_{2}$ e $G_{2}$ é filtro. Resta $q \leq p$, e então $p \in G_{1}$.

Teorema 3.2.25. Sejam $\mathbb{P}, \mathbb{Q}$ noções de forcing e $i: \mathbb{P} \rightarrow \mathbb{Q}$ um mergulho denso. Então

1. se $G$ é um filtro $\mathbb{P}$-genérico, então $H \doteq\{q \in \mathbb{Q} \mid \exists p \in G(i(p) \leq q)\}$ é um filtro $\mathbb{Q}$-genérico e $G=i^{-1}(H)$. Além disso, $\mathrm{V}[G]=\mathrm{V}[H]$.

2. se $H$ é um filtro $\mathbb{Q}$-genérico, então $G \doteq i^{-1}(H)$ é um filtro $\mathbb{P}$-genérico e $H=\{q \in \mathbb{Q} \mid \exists p \in$ $G(i(p) \leq q)\}$. Além disso, $\mathrm{V}[G]=\mathrm{V}[H]$.

3. $p \Vdash_{\mathbb{P}} \varphi\left(x_{1}, \ldots, x_{n}\right) \leftrightarrow i(p) \Vdash_{\mathbb{Q}} \varphi\left(i_{*}\left(x_{1}\right), \ldots, i_{*}\left(x_{1}\right)\right)$, onde $\varphi\left(v_{1}, \ldots, v_{n}\right)$ é uma $\mathcal{L}_{\in}$-fórmula, $p \in \mathbb{P}$ e $x_{1}, \ldots, x_{n} \in \mathrm{V}$.

Consequentemente, $\mathbb{P} e \mathbb{Q}$ são forcing-equivalentes.

Demonstração.

1. $H$ é claramente um filtro. Para provar que $H$ é $\mathbb{Q}$-genérico, é suficiente provar que $H \cap D \neq \emptyset$ para todo $D \subseteq \mathbb{Q}$ aberto denso. Fixe tal $D$. Como $i$ é um mergulho denso, então $i^{-1}(D)$ é predenso e aberto, e portanto denso em $\mathbb{P}$. Assim, se $p \in G \cap i^{-1}(D)$, então $i(p) \in H \cap D$. $G \subseteq i^{-1}(H)$ é trivial; como $G$ é genérico por hipótese e $i^{-1}(H)$ é genérico pelo Lema 3.2.22, segue pelo Lema 3.2.24 que $G=i^{-1}(H)$. Além disso, $\mathrm{V}[G] \subseteq \mathrm{V}[H]$ também pelo Lema 3.2.22. Por outro lado, como $i, G \in \mathrm{V}[G]$, então $\mathrm{V}[H] \subseteq \mathrm{V}[G]$. Segue que $\mathrm{V}[G]=\mathrm{V}[H]$.

2. Segue trivialmente do Lema 3.2.22 e do ítem anterior.

3. Seja $G$ um filtro $\mathbb{P}$-genérico e $H$ como no item 1 . Temos que $\mathrm{V}[G]=\mathrm{V}[H]$, então

$$
\varphi^{\mathrm{V}[G]}\left(x_{1}^{G}, \ldots, x_{n}^{G}\right) \leftrightarrow \varphi^{\mathrm{V}[H]}\left(x_{1}^{H}, \ldots, x_{n}^{H}\right) .
$$

Como $x_{i}^{H}=i_{*}\left(x_{i}\right)^{G}$, o resultado segue pela definição de $\Vdash$.

Tornou-se útil no decorrer do desenvolvimento do forcing o uso de álgebras de Boole completas ao invés de ordens parciais, não só por causa da riqueza da teoria das álgebras de Boole, mas também porque o forcing com álgebras de Boole completas pode ser visto como a construção de um modelo a valores booleanos - isto é, um modelo onde os valores de verdade para as sentenças estão entre 0 e 1, ao invés de simplesmente \{Verdadeiro, Falso\}.

Definição 3.2.26. Sejam $\mathbb{B}$ uma álgebra de Boole completa e $\varphi$ uma $\mathcal{L}_{\in}$ fórmula. Definimos

$$
\llbracket \varphi \rrbracket \doteq \bigvee\{p \in \mathbb{B} \mid p \Vdash \varphi\} .
$$


Definição 3.2.27. Seja $X$ um espaço topológico não vazio. Definimos a álgebra dos abertos regulares, denotada por $\operatorname{ro}(X)$, de $X$ como sendo o conjunto de todos os abertos regulares de $X$ (i.e., abertos $O \subseteq X$ tais que $O=\operatorname{int}(\operatorname{cl}(O))$ ), com as seguintes interpretações:

- $\leq=\subseteq$.

- $\wedge=\cap$.

- $0=\emptyset$.

- $1=X$.

- $O \vee U \doteq \operatorname{int}(\operatorname{cl}(U \cup V))$.

- $O^{\prime}=\operatorname{int}(X \backslash O)$.

Lema 3.2.28. Seja $X$ um espaço topológico não vazio. Então ro $(X)$ é uma álgebra de Boole completa.

Teorema 3.2.29. Seja $\mathbb{P}$ uma noção de forcing. Então existem $\mathbb{B}$ uma álgebra de Boole completa e $i: \mathbb{P} \rightarrow \mathbb{B} \backslash\{0\}$ um mergulho denso.

Demonstração. O "completamento" de $\mathbb{P}$ é a álgebra dos abertos regulares, com a topologia da ordem: $\mathbb{B} \doteq \operatorname{ro}(\mathbb{P})$. O mergulho denso é definido por $i(p) \doteq \operatorname{int}(\operatorname{cl}(\overleftarrow{p}))$. Vamos ver que $i$ é de fato um mergulho denso.

- $i(\mathbb{1})=\mathbb{P}=1$.

- Sejam $p, q \in \mathbb{P}$ tais que $p \leq q$, então $i(p)=\operatorname{int}(\operatorname{cl}(\overleftarrow{p})) \subseteq \operatorname{int}(\operatorname{cl}(\overleftarrow{q}))=i(q)$

- Sejam $p, q \in \mathbb{P}$.

$$
\begin{aligned}
p \perp q & \leftrightarrow \overleftarrow{p} \cap \overleftarrow{q}=\emptyset \\
& \stackrel{*}{\leftrightarrow} \overleftarrow{p} \cap \operatorname{int}(\operatorname{cl}(\overleftarrow{q}))=\emptyset \\
& \stackrel{*}{\leftrightarrow} \operatorname{int}(\operatorname{cl}(\overleftarrow{p})) \cap \operatorname{int}(\operatorname{cl}(\overleftarrow{p}))=\emptyset \\
& \leftrightarrow i(p) \wedge i(q)=0 \\
& \leftrightarrow i(p) \perp i(q)
\end{aligned}
$$

Onde $\stackrel{*}{\leftrightarrow}$ vale pois $\overleftarrow{p}$ e $\operatorname{int}(\operatorname{cl}(\overleftarrow{q}))$ são abertos

- $i(\mathbb{P})$ é denso em $\mathbb{B} \backslash\{0\}$.

Seja $O \in \mathbb{B} \backslash\{0\}$, i.e., $O$ é um aberto regular não-vazio e fixe $p \in O$. Como $\overleftarrow{p} \subseteq O$, $i(p)=\operatorname{int}(\operatorname{cl}(\overleftarrow{p})) \subseteq \operatorname{int}(\operatorname{cl}(O))=O . \operatorname{Logo}, i(p) \leq O$

Além das álgebras de Boole completas, o forcing com noções separativas também é frequentemente útil. As noções separativas de forcing são sempre isomorfas a um subconjunto denso de seu completamento booleano, e felizmente é sempre possível mergulhar densamente uma noção de forcing em uma noção de forcing separativa.

Definição 3.2.30. Seja $\mathbb{P}$ uma noção de forcing. Dizemos que $\mathbb{P}$ é separativa se

$$
\forall p, q \in \mathbb{P}(p \not \leq q \rightarrow \exists r \leq p(r \perp q)) .
$$


Definição 3.2.31. Seja $\mathbb{P}$ uma noção de forcing.

- Definimos $\sim$ uma relação de equivalência em $\mathbb{P}$ estipulando para $p, q \in \mathbb{P}$,

$$
p \sim q \leftrightarrow \forall r \in \mathbb{P}(r\|p \leftrightarrow r\| q)
$$

- Para $p \in \mathbb{P}$, denotamos a classe de equivalência de $p$ por $[p]$.

- O quociente separativo de $\mathbb{P}$ é o quociente $\mathbb{P} / \sim$.

Teorema 3.2.32. Seja $\mathbb{P}$ uma noção de forcing. Então o mapa $p \mapsto[p]$ é um mergulho denso de $\mathbb{P}$ em $\mathbb{P} / \sim$.

Encerramos esta seção com a propriedade de homogeneidade fraca para noções de forcing. Essa propriedade é uma condição suficiente para que qualquer fórmula verdadeira na extensão genérica seja forçada por qualquer condição. Isso significa que se uma fórmula é verdadeira em alguma extensão genérica, então ela será verdadeira em todas as extensões genéricas que são geradas pela mesma noção de forcing.

Definição 3.2.33. Seja $\mathbb{P}$ noção de forcing. $\mathbb{P}$ é dita fracamente homogênea se para quaisquer $p, q \in \mathbb{P}$, existe um automorfismo e de $\mathbb{P}$ tal que $e(p)$ e q são compativeis.

Teorema 3.2.34 (Lei 0-1). Seja $\mathbb{P}$ uma noção de forcing. Se $\mathbb{P}$ é fracamente homogênea, então para qualquer fórmula $\varphi\left(v_{1}, \ldots, v_{n}\right)$ e $x_{1}, \ldots, x_{n} \in V$, temos que ou $\Vdash \varphi\left(x_{1}, \ldots, x_{n}\right)$ ou $\Vdash \neg \varphi\left(x_{1}, \ldots, x_{n}\right)$.

Demonstração. Sejam $\varphi\left(v_{1}, \ldots, v_{n}\right)$ uma fórmula e $x_{1}, \ldots, x_{n}$. Suponha que não ocorre o que queremos provar. Sejam então $p, q$ condições tais que $p \Vdash \neg \varphi\left(x_{1}, \ldots, x_{n}\right)$ e $q \Vdash \varphi\left(x_{1}, \ldots, x_{n}\right)$, e seja $e$ um automorfismo de $\mathbb{P}$ tal que $e(p) \| q$. Assim, $e(p) \Vdash \neg \varphi\left(x_{1}, \ldots, x_{n}\right)$, o que contraria $e(p) \| q$.

\subsection{Um mínimo sobre forcing iterado}

O objetivo dessa seção é simples de ser entendido: se $\mathbb{P}$ é uma noção de forcing e $G_{0}$ é um filtro $\mathbb{P}$-genérico, podemos formar a extensão $\mathrm{V}\left[G_{0}\right]$. Agora, se $\mathbb{Q}$ é outra noção de forcing em $\mathrm{V}\left[G_{0}\right]$ e $G_{1}$ é um filtro $\mathbb{Q}$-genérico sobre $\mathrm{V}\left[G_{0}\right]$, o procedimento para adicionar construir a extensão $\mathrm{V}\left[G_{0}\right]\left[G_{1}\right]$ é igual ao que fizemos nas seções anteriores, agora partindo de $\mathrm{V}\left[G_{0}\right]$. É natural então perguntar

Existe alguma noção de forcing $\mathbb{R}$ tal que para algum $G$ filtro $\mathbb{R}$-genérico, $\mathrm{V}[G]=$ $\mathrm{V}\left[G_{0}\right]\left[G_{1}\right]$ ?

Essa noção de forcing $\mathbb{R}$ deve ser uma combinação entre a noção de forcing $\mathbb{P}$ e o $\mathbb{P}$-nome $\dot{\mathbb{Q}}$, para a noção de forcing que vive em $\mathrm{V}\left[G_{0}\right]$. Começamos a nossa análise observando que simplesmente forçar com $\mathbb{P} \times \mathbb{Q}$ adiciona tanto um filtro $\mathbb{P}$-genérico, quanto um filtro $\mathbb{Q}$-genérico.

Definição 3.3.1. Sejam $\left(\mathbb{P}, \leq_{\mathbb{P}}, \mathbb{1}_{\mathbb{P}}\right),\left(\mathbb{Q}, \leq_{\mathbb{Q}}, \mathbb{1}_{\mathbb{Q}}\right)$ noções de forcing. Podemos considerar o forcing produto $(\mathbb{P} \times \mathbb{Q}, \leq, \mathbb{1})$, onde $\mathbb{1}=\left(\mathbb{1}_{\mathbb{P}}, \mathbb{1}_{\mathbb{Q}}\right) e$

$$
\left(p_{0}, q_{0}\right) \leq\left(p_{1}, q_{1}\right) \leftrightarrow p_{0} \leq_{\mathbb{P}} p_{1} \wedge q_{0} \leq_{\mathbb{Q}} q_{1} .
$$

Lema 3.3.2. Sejam $\mathbb{P}$ e $\mathbb{Q}$ noções de forcing, e os mapas $i_{0}: \mathbb{P} \rightarrow \mathbb{P} \times \mathbb{Q}$ e $i_{1}: \mathbb{Q} \rightarrow \mathbb{P} \times \mathbb{Q}$ com $i_{0}(p)=\left(p, \mathbb{1}_{\mathbb{P}}\right)$ e $i_{1}(q)=\left(\mathbb{1}_{\mathbb{Q}}, q\right)$. Seja $G$ um filtro $\mathbb{P} \times \mathbb{Q}$-genérico e, em $\mathrm{V}[G]$, considere $G_{0}=i_{0}^{-1}(G)$ e $G_{1}=i_{1}^{-1}(G)$. Então $G_{0}$ é $\mathbb{P}$-genérico, $G_{1}$ é $\mathbb{Q}$-genérico e $G=G_{0} \times G_{1}$. 
Demonstração. Como $i_{0}$ e $i_{1}$ são mergulhos completos, segue pelo Lema 3.2.22 que $G_{0}$ e $G_{1}$ são genéricos para as suas respectivas noções de forcing. Agora, seja $(p, q) \in G$. Como $(p, q) \leq\left(p, \mathbb{1}_{\mathbb{Q}}\right),\left(\mathbb{1}_{\mathbb{P}}, q\right)$ e $G$ é um filtro em $\mathbb{P} \times \mathbb{Q}$, então $\left(p, \mathbb{1}_{\mathbb{Q}}\right)=i_{0}(p) \in G$ e $\left(\mathbb{1}_{\mathbb{P}}, q\right)=i_{1}(q) \in G$. Logo, $(p, q) \in G_{0} \times G_{1}$.

Reciprocamente, seja $(p, q) \in G_{0} \times G_{1}$. Assim, $i_{0}(p)=\left(p, \mathbb{1}_{\mathbb{Q}}\right) \in G$ e $i_{1}(q)=\left(\mathbb{1}_{\mathbb{P}}, q\right) \in G$. Assim, seja $(r, s) \in G$ tal que $(r, s) \leq\left(p, \mathbb{1}_{\mathbb{Q}}\right),\left(\mathbb{1}_{\mathbb{P}}, q\right)$. Então $(r, s) \leq(p, q)$ e, como $G$ é filtro, então $(p, q) \in G$

Teorema 3.3.3. Sejam $\mathbb{P}, \mathbb{Q}$ noções de forcing e, fora de $V, G_{0} \subseteq \mathbb{P}, G_{1} \subseteq \mathbb{Q}$. São equivalentes:

1. $G_{0} \times G_{1}$ é $\mathbb{P} \times \mathbb{Q}$-genérico.

2. $G_{0}$ é $\mathbb{P}$-genérico e $G_{1}$ é $\mathbb{Q}$-genérico sobre $\mathrm{V}\left[G_{0}\right]$.

3. $G_{1}$ é $\mathbb{Q}$-genérico e $G_{0}$ é $\mathbb{P}$-genérico sobre $\mathrm{V}\left[G_{1}\right]$.

Além disso, se uma das condições acima é satisfeita, $\mathrm{V}\left[G_{0} \times G_{1}\right]=\mathrm{V}\left[G_{0}\right]\left[G_{1}\right]=\mathrm{V}\left[G_{1}\right]\left[G_{0}\right]$.

Demonstração.

- (1. $\Rightarrow 2$.) Já sabemos que $G_{1}$ é $\mathbb{Q}$-genérico (sobre $V$ ). Agora precisamos mostrar que $G_{1}$ intersecta todos os densos de $\mathrm{V}\left[G_{0}\right]$ também. Seja então, em $\mathrm{V}\left[G_{0}\right]$, um denso $D \subseteq \mathbb{Q}$. Sejam $\dot{D}$ um $\mathbb{P}$-nome para $D$ e $p_{0} \in G_{0}$ tais que $p_{0} \Vdash(\dot{D}$ é denso em $\check{\mathbb{Q}})$. Agora seja

$$
D^{\prime}=\left\{(p, q) \in \mathbb{P} \times \mathbb{Q} \mid p \leq p_{0} \wedge p \Vdash \check{q} \in \dot{D}\right\} .
$$

Vamos mostrar que $D^{\prime}$ é denso abaixo de $\left(p_{0}, \mathbb{1}_{\mathbb{Q}}\right)$. De fato, seja $\left(p_{1}, q_{1}\right) \leq\left(p_{0}, \mathbb{1}_{\mathbb{Q}}\right)$. Como $p_{1} \leq p_{0}$, então $p_{1} \Vdash \dot{D}$ é denso em $\check{\mathbb{Q}} \mathrm{e}$, portanto, $p_{1} \Vdash_{\mathbb{P}} \exists x \in \check{\mathbb{Q}}\left(x \in \dot{D} \wedge x \check{\leq} \check{q}_{1}\right)$. Assim, devem existir $p_{2} \leq p_{1}$ e $q_{1} \in \mathbb{Q}$ tais que $p_{2} \Vdash \check{q}_{2} \in \dot{D} \wedge \check{q}_{2} \check{\leq} \check{q}_{1}$. Temos $\left(p_{2}, q_{2}\right) \in D^{\prime}$ e $\left(p_{2}, q_{2}\right) \leq\left(p_{1}, q_{1}\right)$. Agora, como $\left(p_{0}, \mathbb{1}_{\mathbb{Q}}\right) \in G_{0} \times G_{1}$, então $D^{\prime} \cap\left(G_{0} \times G_{1}\right) \neq \emptyset$. Seja $(r, s) \in D^{\prime} \cap\left(G_{0} \times G_{1}\right)$. Temos que $r \in G_{0}, s \in G_{0}$ e $r \Vdash_{\mathbb{P}} \check{s} \in \dot{D}$ e, portanto, $s \in D$. Logo, $s \in G_{1} \cap D$.

- $(2 . \Rightarrow$ 1.) Seja um denso $D \subseteq \mathbb{P} \times \mathbb{Q}$. Considere o conjunto

$$
D^{*}=\left\{q \in \mathbb{Q} \mid \exists p \in G_{0}((p, q) \in D)\right\} .
$$

Vamos mostrar que $D^{*} \in \mathrm{V}\left[G_{0}\right]$ é denso em $\mathbb{Q}$. Seja $q_{0} \in \mathbb{Q}$. Note que o conjunto

$$
\tilde{D}=p \in \mathbb{P} \mid \exists q \leq q_{0}((p, q) \in D)
$$

é denso em $\mathbb{P}$ pois $D$ é denso em $\mathbb{P} \times \mathbb{Q}$. Assim, sejam $p_{1} \in \tilde{D} \cap G_{0}$ e $q_{1} \leq q_{0}$ tais que $\left(p_{1}, q_{1}\right) \in D$. Note que $q_{1} \in D^{*}$, como queríamos. Logo, $D^{*} \cap G_{1} \neq \emptyset$ e, portanto, $D \cap\left(G_{0} \times G_{1}\right) \neq \emptyset$.

O caso 1. $\Leftrightarrow 3$. é análogo. Para verificar a última afirmação, usamos a minimalidade das extensões genéricas: $\mathrm{V}\left[G_{0} \times G_{1}\right] \subseteq \mathrm{V}\left[G_{0}\right]\left[G_{1}\right]$ pois $G_{0} \times G_{1} \in \mathrm{V}\left[G_{0}\right]\left[G_{1}\right]$. Para $\mathrm{V}\left[G_{0}\right]\left[G_{1}\right] \subseteq \mathrm{V}\left[G_{0} \times G_{1}\right]$, primeiro note que $\mathrm{V}\left[G_{0}\right] \subseteq \mathrm{V}\left[G_{0} \times G_{1}\right]$ pois $G_{0} \in \mathrm{V}\left[G_{0} \times G_{1}\right]$, e a partir disso e de que $G_{1} \in \mathrm{V}\left[G_{0} \times G_{1}\right]$ segue que $\mathrm{V}\left[G_{0}\right]\left[G_{1}\right] \subseteq \mathrm{V}\left[G_{0} \times G_{1}\right]$.

Com esse "aquecimento", voltamos para o problema do início da seção. Vamos desenvolver a teoria de iteração em dois passos.

Definição 3.3.4. Seja $\mathbb{P}$ uma noção de forcing. Um $\mathbb{P}$-nome para uma noção de forcing é uma tripla de $\mathbb{P}$-nomes $\left(\dot{\mathbb{Q}}, \dot{\leq}_{\mathbb{Q}}, \dot{1}_{\mathbb{Q}}\right)$ tal que

$$
\vdash_{\mathbb{P}} \dot{\mathbb{1}}_{\mathbb{Q}} \in \dot{\mathbb{Q}} \wedge \dot{\leq}_{\mathbb{Q}} \text { é uma ordem parcial em } \dot{\mathbb{Q}} \text { com maior elemento } \dot{\mathbb{1}}_{\mathbb{Q}} \text {. }
$$

Frequentemente escreveremos simplesmente $\dot{\mathbb{Q}}$ ao invés da tripla $\left(\dot{\mathbb{Q}}, \dot{\leq}_{\mathbb{Q}}, \dot{\mathbb{1}}_{\mathbb{Q}}\right)$. 
Definição 3.3.5. Sejam $\mathbb{P}$ uma noção de forcing e $\mathbb{\mathbb { Q }}$ um $\mathbb{P}$-nome para uma noção de forcing. Definimos o produto $(\mathbb{P} * \dot{\mathbb{Q}}, \leq, \mathbb{1})$ como sendo

- $\mathbb{P} * \dot{\mathbb{Q}} \doteq\{(p, \dot{q}) \in \mathbb{P} \times \operatorname{dom}(\dot{\mathbb{Q}}) \mid p \Vdash \dot{q} \in \dot{\mathbb{Q}}\}$.

- $\left(p_{1}, \dot{q}_{1}\right) \leq\left(p_{2}, \dot{q}_{2}\right) \leftrightarrow p_{1} \leq_{\mathbb{P}} p_{2} \wedge p_{1} \Vdash \dot{q}_{1} \dot{\leq}_{\mathbb{Q}} \dot{q}_{2}$.

- $\mathbb{1}=\left(\mathbb{1}_{\mathbb{P}}, \dot{\mathbb{1}}_{\mathbb{Q}}\right)$.

O mergulho canônico de $\mathbb{P}$ em $\mathbb{P} * \dot{\mathbb{Q}}$ é o mapa $i: \mathbb{P} \rightarrow \mathbb{P} * \dot{\mathbb{Q}}$ definido por $i(p)=\left(p, \dot{\mathbb{1}}_{\mathbb{Q}}\right)$. $\dashv$

Lema 3.3.6. Sejam $\mathbb{P}$ uma noção de forcing e $\dot{\mathbb{Q}}$ um $\mathbb{P}$-nome para uma noção de forcing. Então $i$, o mergulho canônico de $\mathbb{P}$ em $\mathbb{P} * \dot{\mathbb{Q}}$, é um mergulho completo.

Definição 3.3.7. Sejam $\mathbb{P}$ uma noção de forcing e $\dot{\mathbb{Q}}$ um $\mathbb{P}$-nome para uma noção de forcing. Agora, sejam $G_{0}$ um filtro $\mathbb{P}$-genérico e em $\mathrm{V}[G]$ e $G_{1} \subseteq \dot{\mathbb{Q}}^{G_{0}}$. Definimos

$$
G_{0} * G_{1} \doteq\left\{(p, \dot{q}) \in \mathbb{P} * \dot{\mathbb{Q}} \mid p \in G_{0} \wedge \dot{q}^{G_{0}} \in G_{1}\right\} .
$$

Definição 3.3.8. Sejam $\mathbb{P}$ uma noção de forcing, $\mathbb{Q}$ um $\mathbb{P}$-nome para uma noção de forcing, $G$ um filtro $\mathbb{P} * \dot{\mathbb{Q}}$-genérico e $i$ o mergulho canônico de $\mathbb{P}$ em $\mathbb{P} * \dot{\mathbb{Q}}$. Defina os seguintes conjuntos

- $G_{0} \doteq i^{-1}(G), e$

- $G_{1} \doteq\left\{\dot{q}^{G_{0}} \mid \dot{q} \in \operatorname{dom}(\dot{\mathbb{Q}}) \wedge \exists p((p, \dot{q}) \in G)\right\}$.

Teorema 3.3.9. Sejam $\mathbb{P}$ uma noção de forcing, $\mathbb{Q}$ um $\mathbb{P}$-nome para uma noção de forcing e $G$ um filtro $\mathbb{P} * \dot{\mathbb{Q}}$-genérico. Então

1. G $G_{0}$ é $\mathbb{P}$-genérico.

2. $G_{1}$ é $\dot{\mathbb{Q}}^{G_{0}}$-genérico sobre $\mathrm{V}\left[G_{0}\right]$ (i.e., $\left(\dot{\mathbb{Q}}^{G_{0}}, \dot{\leq}_{\mathbb{Q}}^{G_{0}}, \dot{\mathbb{1}}_{\mathbb{Q}}^{G_{0}}\right)$-genérico sobre $\mathrm{V}\left[G_{0}\right]$ ).

3. $G=G_{0} * G_{1} e \mathrm{~V}[G]=\mathrm{V}\left[G_{0}\right]\left[G_{1}\right]$.

Demonstração.

1. Segue diretamente do Lema 3.2.22.

2 .

- $G_{1} \subseteq \dot{\mathbb{Q}}^{G_{0}}$.

Sejam $\dot{q} \in \operatorname{dom}(\dot{\mathbb{Q}})$ e $p \in \mathbb{P}$ tais que $(p, \dot{q}) \in G$ (i.e., $\left.\dot{q}^{G_{0}} \in G_{1}\right)$. Por um lado, $(p, \dot{q}) \in G$ implica $p \Vdash \dot{q} \in \dot{\mathbb{Q}}$. Por outro lado, $(p, \dot{q}) \in G$ implica $\left(p, \dot{\mathbb{1}}_{\mathbb{Q}}\right) \in G$, o que implica $p \in i^{-1}(G)=G_{0}$. Pelo Teorema Fundamental do Forcing, $\dot{q}^{G_{0}} \in \mathbb{\mathbb { Q }}^{G_{0}}$, como queríamos.

- Provar que $\dot{\mathbb{1}}_{\mathbb{Q}}^{G_{0}} \in G_{1}$ e que $G_{1}$ é conectado é apenas notacionalmente complicado, mas segue simplesmente de $G$ ser conectado.

- $G_{1}$ é fechado pra cima.

Sejam $\dot{q}^{G_{0}} \in G_{1}$ e $p \in G_{0}$ tais que $\dot{q} \in \operatorname{dom}(\dot{\mathbb{Q}})$ e $(p, \dot{q}) \in G$. Seja $\dot{r}^{G_{0}} \in \dot{\mathbb{Q}}^{G_{0}}$ tal que $\dot{q}^{G_{0}} \leq \dot{r}^{G_{0}}$ (estamos abreviando $\dot{\leq}_{\mathbb{Q}}^{G_{0}}$ para $\leq$, simplesmente). Pelo Lema da Verdade, existe $p^{\prime} \in G_{0}$ tal que $p^{\prime} \Vdash \dot{q} \dot{\dot{r}}$. Agora, como $\left(p^{\prime}, \dot{\mathbb{1}}_{\mathbb{Q}}\right) \in G$ e $(p, \dot{q}) \in G$, então podemos tomar $\left(p^{\prime \prime}, \dot{s}\right) \in G$ com $\left(p^{\prime \prime}, \dot{s}\right) \leq\left(p^{\prime}, \dot{\mathbb{1}}_{\mathbb{Q}}\right),(p, \dot{q})$, donde segue por definição que $p^{\prime \prime} \Vdash \dot{s} \dot{\dot{q}}$. Assim, combinando as informações, temos que $p^{\prime \prime} \Vdash \dot{q} \dot{\leq} \dot{r}$, e então $p^{\prime \prime} \Vdash \dot{s} \dot{\leq} \dot{r}$. Segue que $\left(p^{\prime \prime}, \dot{s}\right) \leq\left(p^{\prime}, \dot{r}\right)$, então $\left(p^{\prime}, \dot{r}\right) \in G$, e pela definição de $G_{1}$ temos que $\dot{r}^{G_{0}} \in G_{1}$. 
- $G_{1}$ intersecta todos os $\dot{\mathbb{Q}}^{G_{0}}$-densos de $\mathrm{V}\left[G_{0}\right]$.

Seja $D \subseteq \dot{\mathbb{Q}}^{G_{0}}$ denso tal que $D \in \mathrm{V}\left[G_{0}\right]$. Sejam $\dot{D}$ um $\mathbb{P}$-nome para $D$ e $p_{0} \in G_{0}$ tais que $p_{0} \Vdash(\dot{D}$ é denso em $\dot{\mathbb{Q}})$. Agora seja

$$
D^{\prime}=\left\{(p, \dot{q}) \in \mathbb{P} * \dot{\mathbb{Q}} \mid p \leq p_{0} \wedge p \Vdash \dot{q} \in \dot{D}\right\} .
$$

Vamos mostrar que $D^{\prime}$ é denso abaixo de $\left(p_{0}, \dot{\mathbb{1}}_{\mathbb{Q}}\right)$. De fato, seja $\left(p_{1}, \dot{q}_{1}\right) \leq\left(p_{0}, \dot{\mathbb{1}}_{\mathbb{Q}}\right)$. Como $p_{1} \leq p_{0}$, então $p_{1} \Vdash(\dot{D}$ é denso em $\dot{\mathbb{Q}})$ e, portanto, $p_{1} \Vdash \exists x \in \dot{\mathbb{Q}}\left(x \in \dot{D} \wedge x \leq \dot{q}_{1}\right)$. Assim, devem existir $p_{2} \leq p_{1}$ e $\dot{q}_{1} \in \operatorname{dom}(\mathbb{Q})$ tais que $p_{2} \Vdash\left(\dot{q}_{2} \in \dot{D} \wedge \dot{q}_{2} \dot{\leq}_{1} \dot{q}_{1}\right)$. Então $\left(p_{2}, \dot{q}_{2}\right) \in D^{\prime}$ e $\left(p_{2}, \dot{q}_{2}\right) \leq\left(p_{1}, \dot{q}_{1}\right)$. Agora, como $\left(p_{0}, \dot{\mathbb{1}}_{\mathbb{Q}}\right) \in G$, então $D^{\prime} \cap G \neq \emptyset$. Seja então $(r, \dot{s}) \in D^{\prime} \cap G$. Temos que $r \in G_{0}, \dot{s}^{G_{0}} \in G_{0}$ e $r \Vdash \dot{s} \in \dot{D}$ e, portanto, $\dot{s}^{G_{0}} \in D$. Logo, $\dot{s}^{G_{0}} \in G_{1} \cap D$.

3. $\bullet G=G_{0} * G_{1}$.

Se $(p, \dot{q}) \in G$, então $p \in G_{0}$ e $\dot{q}^{G_{0}} \in G_{1}$, portanto $(p, \dot{q}) \in G_{0} * G_{1}$. Reciprocamente, se $(p, \dot{q}) \in G_{0} * G_{1}$, então $(p, \dot{q}) \in \mathbb{P} * \dot{\mathbb{Q}}, p \in G_{0}, \dot{q}^{G_{0}} \in G_{1}$. Assim, $\left(p, \dot{\mathbb{1}}_{\mathbb{Q}}\right) \in G$ e $\left(p^{\prime}, \dot{q}\right) \in G$, para algum $p^{\prime}$. Seja $\left(p^{\prime \prime}, \dot{r}\right) \in G$ tal que $\left(p^{\prime \prime}, \dot{r}\right) \leq\left(p, \dot{\mathbb{1}}_{\mathbb{Q}}\right),\left(p^{\prime}, \dot{q}\right)$. Então $p^{\prime \prime} \leq p, p^{\prime}$ e $p^{\prime \prime} \Vdash \dot{r} \dot{\dot{q}} \dot{q}$. Assim, $\left(p^{\prime \prime}, \dot{r}\right) \leq(p, \dot{q})$ e, portanto, $(p, \dot{q}) \in G$. Concluimos que $G=G_{0} * G_{1}$.

- $\mathrm{V}[G]=\mathrm{V}\left[G_{0}\right]\left[G_{1}\right]$.

$\mathrm{V}[G] \subseteq \mathrm{V}\left[G_{0}\right]\left[G_{1}\right]$ pois $G=G_{0} * G_{1} \in \mathrm{V}\left[G_{0}\right]\left[G_{1}\right]$, e $\mathrm{V}\left[G_{0}\right]\left[G_{1}\right] \subseteq \mathrm{V}[G]$ pois $G_{0} \in \mathrm{V}[G]$, e então $\mathrm{V}\left[G_{0}\right]\left[G_{1}\right] \subseteq \mathrm{V}[G]$ pois $G_{1} \in \mathrm{V}[G]$. Concluimos que $\mathrm{V}[G]=\mathrm{V}\left[G_{0}\right]\left[G_{1}\right]$.

Encerramos esta seção com comentários sobre modelos intermediários entre o modelo inicial e sua extensão por forcing.

Teorema 3.3.10. Sejam $\mathbb{R}$ uma noção de forcing, $G$ um filtro $\mathbb{R}$-genérico e $N$ um modelo canônico de $\mathrm{ZFC}$ tal que

$$
V \subseteq N \subseteq \mathrm{V}[G] .
$$

Então existe uma noção de forcing $\mathbb{P}$ e um $\mathbb{P}$-nome para uma noção de forcing $\dot{\mathbb{Q}}$ tais que $\mathbb{R}$ e $\mathbb{P} * \dot{\mathbb{Q}}$ são isomorfos, e identificando $\mathbb{R}$ com $\mathbb{P} * \dot{\mathbb{Q}}$, temos $N=\mathrm{V}\left[G_{0}\right]$ e $\mathrm{V}[G]=\mathrm{V}\left[G_{0}\right]\left[G_{1}\right]$.

Teorema 3.3.11. Sejam $\mathbb{R}$ uma noção de forcing, $G$ um filtro $\mathbb{R}$-genérico e $S \subseteq$ ON um conjunto de ordinais tal que $S \in \mathrm{V}[G]$. Então existem $\mathbb{O}$ uma noção de forcing e $K$ um filtro $\mathbb{O}$-genérico tal que

- $S \in \mathrm{V}[K]$;

- se $N$ é um modelo canônico transitivo de ZFC com $S \in N$, então $\mathrm{V}[K] \subseteq N$;

- $V \subseteq \mathrm{V}[K] \subseteq \mathrm{V}[G]$

Denotamos esse modelo $\mathrm{V}[K]$ por $\mathrm{V}[S]$. Além disso, existem $\mathbb{P} \in \mathrm{V}[S]$ e $H$ um filtro $\mathbb{P}$-genérico sobre $\mathrm{V}[S]$ tais que $\mathrm{V}[G]=\mathrm{V}[S][H]$.

Embora haja menção em algumas partes desse texto a iterações transfinitas de forcing, não falaremos disso aqui. As referências a esse tipo de forcing iterado serão feitas informalmente. Encerramos essa seção com um breve comentário sobre o forcing próprio, que é um conceito central para os argumentos envolvendo as classes de forcing consideradas neste texto.

É mais difícil achar a parte histórica e motivacional para o forcing próprio do que alguém esperaria. Essa parte da pesquisa foi feita basicamente com o auxílio do Math Overflow. Parece ser um consenso que é particularmente difícil fazer uma pesquisa histórica de objetos e conceitos que estão muito próximos da contemporaneidade, como é o caso do forcing próprio, introduzido por Shelah pela primeira vez em 1980 [She80] — sem a prova do Teorema de Iteração para suporte 
enumerável - utilizado para obter resultados de independência com relação a números de modelos não isomorfos.

Anterior ao artigo [She80], Laver em 1976 [Lav76] utilizou de uma técnica de forcing e uma iteração com suporte enumerável para resolver a Conjectura de Borel (Veja a Seção 4.5). Ao que se sabe, esse foi o primeiro uso "importante" de uma iteração com suporte enumerável. Em 1982 [She82] Shelah reintroduziu o forcing próprio com a demonstração do Teorema de Iteração e diversos outros resultados de preservação. Logo veremos que a propriedade "ser próprio" para uma noção de forcing é indicativa de bom comportamento, em algum sentido. Não surpreendentemente, o forcing introduzido por Laver também tem essa propriedade (i.e., é forcing próprio).

A propriedade de "ser próprio" para noções de forcing é preservada por iterações com suporte enumerável, e essa é uma das propriedades boas. Shelah definiu o forcing próprio como sendo um forcing que preserva - em qualquer extensão genérica - conjuntos estacionários de $[\kappa] \leq \aleph_{0}$, para todo cardinal $\kappa$. Nossa definição de forcing próprio será uma definição que utiliza submodelos elementares, mais adequada para o propósito desse texto. A ideia principal é que para uma noção de forcing $\mathbb{P}$, é possível encapsular o argumento de forcing em uma "miniatura" do universo V (isso lembra a ideia de considerar modelos para fragmentos suficientemente grandes de ZFC). Se $\mathbb{P}$ é uma noção de forcing e $\kappa=|\mathbb{P}|$, se $\lambda>2^{\kappa}$, então $H_{\lambda}$ é um modelo para ZFC $-\mathrm{P}$, que contém uma cópia de $\mathbb{P}$ e $\mathcal{P}(\mathbb{P})$. Embora $H_{\lambda}$ não satisfaça necessariamente o Axioma das Partes, $H_{\lambda}$ tem informação suficiente para definir a relação de forcing $\Vdash$ (que na verdade é $\Vdash_{\mathbb{P}}^{*}$, mas sempre omitiremos o asterisco e $\mathbb{P}$ quando for claro).

Definição 3.3.12. Sejam $\mathbb{P}$ uma noção de forcing, $M \preceq H_{\lambda}$ um submodelo elementar enumerável, com $\lambda>2^{|\mathbb{P}|}$ tal que $\mathbb{P} \in M$ e $G$ um filtro $\mathbb{P}$-genérico (sobre V). Definimos

- $\pi: M \rightarrow \bar{M}$ o colapso transitivo da estrutura $M$;

- se $x \in M, \bar{x} \doteq \pi(x)$;

- $\bar{G} \doteq \pi[G]$.

Dizemos que $G$ é um filtro $\mathbb{P}$-genérico sobre $M$ se $\bar{G}$ é um filtro $\overline{\mathbb{P}}$-genérico sobre $\bar{M}$. Nesse caso, $\bar{M}[\bar{G}]$ é uma extensão genérica de $\bar{M}$. Definimos

$$
M[G] \doteq \bar{M}[\bar{G}] .
$$

Lema 3.3.13. Sejam $\mathbb{P}$ uma noção de forcing, $M \preceq H_{\lambda}$ um submodelo elementar enumerável, com $\lambda>2^{|\mathbb{P}|}$ tal que $\mathbb{P} \in M$ e $G$ um filtro $\mathbb{P}$-genérico. Então $G$ é um filtro $\mathbb{P}$-genérico sobre $M$ se para todo $D \in M,(D \text { é denso em } \mathbb{P})^{M}$ então

$$
G \cap D \cap M \neq \emptyset .
$$

Teorema 3.3.14. Sejam $\mathbb{P}$ uma noção de forcing, $M \preceq H_{\lambda}$, com $\lambda>2^{|\mathbb{P}|}$ tal que $\mathbb{P} \in M$, $\varphi\left(v_{1}, \ldots, v_{n}\right)$ uma $\mathcal{L}_{\in}$-fórmula e $x_{1}, \ldots, x_{n} \in M$. Então

$$
\varphi^{M[G]}\left(\bar{x}_{1}^{G}, \ldots, \bar{x}_{n}^{G}\right) \leftrightarrow \exists p \in G \cap M\left(p \Vdash \varphi\left(x_{1}, \ldots, x_{n}\right)\right)^{M} .
$$

Definição 3.3.15. Sejam $\mathbb{P}$ uma noção de forcing e $M \preceq H_{\lambda}$, com $\lambda>2^{|\mathbb{P}|}$ tal que $\mathbb{P} \in M$. Uma condiçấo $p \in \mathbb{P}$ é dita $(M, \mathbb{P})$-master se para todo $D \in M$ tal que $(D \text { é denso em } \mathbb{P})^{M}$,

$$
p \Vdash \check{M} \cap \check{D} \cap \dot{G} \neq \emptyset .
$$

Ou, equivalentemente, 
Definição 3.3.16. Sejam $\mathbb{P}$ uma noção de forcing. Dizemos que $\mathbb{P}$ é forcing próprio se, e somente se, para todo cardinal $\lambda>2^{|\mathbb{P}|}$ e $M \preceq H_{\lambda}$ enumerável com $\mathbb{P} \in M$, toda condição $p \in \mathbb{P} \cap M$ tem uma extensão $q \leq p$ que é $(M, \mathbb{P})$-master. 


\section{Capítulo 4}

\section{Dualidade entre regularidade e forcing}

\subsection{Plano geral}

No Capítulo 1 introduzimos três noções de regularidade na reta: Lebesgue mensurabilidade (Definição 1.4.6), Baire mensurabilidade (Definição 1.4.2) e a propriedade do conjunto perfeito (Definição 1.2.3). Mostraremos que as propriedades de Lebesgue e de Baire se enquadram em um contexto de regularidade, que chamaremos de $\mathbb{P}_{J}$-mensurabilidade (Definição 5.1.14), onde é possível provar muitos resultados uniformente. A propriedade do conjunto perfeito, por outro lado, se enquadra em um contexto diferente, que chamaremos de $\mathbb{P}_{J}$-dicotomia (Definição 5.1.10). Tanto a $\mathbb{P}_{J}$-mensurabilidade quanto a $\mathbb{P}_{J}$-dicotomia representam formas de regularidade. Além disso, se um conjunto satisfaz a $\mathbb{P}_{J}$-dicotomia, então esse conjunto é $\mathbb{P}_{J}$-mensurável, sendo que a recíproca em geral não é verdadeira. Por alguma razão, a $\mathbb{P}_{J}$-dicotomia acaba se comportando de forma muito mais misteriosa do que a $\mathbb{P}_{J}$-mensurabilidade. Isto é, não é fácil generalizar os resultados obtidos para $\mathbb{P}_{J}$-mensurabilidade, para a $\mathbb{P}_{J}$-dicotomia.

Existe uma relação intrínseca entre as noções de regularidade abordadas neste texto e as noções clássicas de forcing. Sem dúvida, o primeiro trabalho em que é explorada essa dualidade é o artigo [Sol70] de Solovay e falaremos mais disso na Seção 4.2. Assim, dada uma noção de regularidade, a primeira coisa que faremos é verificar qual é a noção de forcing mais natural correspondente, e como caracterizar essa noção de regularidade usando essa noção de forcing. As relações que encontraremos estão resumidas na seguinte tabela:

\begin{tabular}{|c|c|}
\hline Regularidade & forcing clássico \\
\hline \hline Lebesgue mensurabilidade & aleatório $(\mathbb{B})(4.2 .4)$ \\
\hline Baire mensurabilidade & Cohen $(\mathbb{C})$ \\
\hline PCP / Marczewski mensurabilidade $(4.3 .1)$ & Sacks $(\mathbb{S})(4.3 .6)$ \\
\hline Ramsey mensurabilidade $(4.6 .4)$ & Mathias $(\mathbb{R})(4.6 .5)$ \\
\hline Completamente doughnut $(4.7 .3)$ & Silver $(\mathbb{V})(4.7 .9)$ \\
\hline$K_{\sigma \text {-dicotomia }(4.4 .5) / \mathbb{M} \text {-mensurabilidade }(4.4 .6)}$ & Miller $(\mathbb{M})(4.4 .7)$ \\
\hline -dicotomia $(4.5 .7) / \mathbb{L}$-mensurabilidade $(4.5 .4)$ & Laver $(\mathbb{L})(4.5 .2)$ \\
\hline
\end{tabular}

Essa dualidade entre regularidade e forcing deve motivar uma noção geral de regularidade para noções de forcing. A primeira abordagem na direção dessa noção geral de regularidade foi considerada em 1994 [Bre94], e continuada em [BL99], onde foi introduzida a noção de forcing arbóreo. Nosso contexto de regularidade generaliza a noção de regularidade para forcings arbóreos, usando a noção de regularidade idealizada, que foi considerada em 2012 [K $\left.\mathrm{K}^{+} 12\right]$. Isso foi feito usando a ideia bastante geral de forcing idealizado (Definição 4.2.5). Apesar da generalidade dessa técnica, nos restringiremos apenas ao contexto da regularidade.

O forcing idealizado foi introduzido e investigado por Zapletal em 2004 [Zap04]. Zapletal percebeu que diversas noções clássicas de forcing são "idealizadas", em um sentido que será preciso. Esse forcing leva esse nome pois ele é obtido a partir de um $\sigma$-ideal $J$, formando o forcing $\mathbb{P}_{J}$ (Definição 
4.2.5). Veremos que as noções clássicas de forcing são equivalentes a forcings idealizados, mostrando que elas podem ser mergulhadas densamente nesses forcings. As relações que encontraremos estão resumidas na seguinte tabela:

\begin{tabular}{|c|c|c|}
\hline Regularidade & forcing clássico & $\sigma$-ideal \\
\hline \hline Lebesgue mensurabilidade & aleatório $(\mathbb{B})$ & $\mathcal{N}(1.4 .7)$ \\
\hline Baire mensurabilidade & Cohen $(\mathbb{C})$ & $\mathcal{M}(1.4 .3)$ \\
\hline PCP / Marczewski mensurabilidade & Sacks $(\mathbb{S})$ & ctbl $(1.2 .4)$ \\
\hline Ramsey mensurabilidade & Mathias $(\mathbb{R})$ & $\mathcal{R}^{0}(4.6 .7)$ \\
\hline Propriedade doughnut & Silver $(\mathbb{V})$ & $\mathcal{D}^{0}(4.7 .4)$ \\
\hline$K_{\sigma}$-dicotomia / M-mensurabilidade & Miller $(\mathbb{M})$ & $K_{\sigma}(4.4 .3)$ \\
\hline$\ell$-dicotomia / L-mensurabilidade & Laver $(\mathbb{L})$ & $\mathcal{L}^{0}(4.5 .6)$ \\
\hline
\end{tabular}

Tabela 4.1: Relação entre noções clássicas de regularidade, forcings clássicos e ideais

Todas as noções de forcing acima compartilham uma propriedade em comum: são forcings próprios. Esse fato será crucial para desenvolver uma noção geral de regularidade. A saber, há uma boa condição necessária e suficiente para que os forcings idealizados sejam próprios (Teorema 5.1.6).

Depois de introduzir a regularidade idealizada, i.e., a $\mathbb{P}_{J}$-mensurabilidade e a $\mathbb{P}_{J}$-dicotomia, verificaremos o Teorema de Solovay (Corolário 5.2.11), somente para o caso da $\mathbb{P}_{J}$-mensurabilidade. Até o momento, parece não haver uma prova de que a $\mathbb{P}_{J}$-dicotomia sempre vale no modelo de Solovay, nem se conhece algum contraexemplo. O Teorema de Solovay é uma teorema da teoria ZFC $+\exists \kappa(\kappa$ é um cardinal inacessível). É natural então perguntar se é possível obter o mesmo teorema, mas em ZFC somente. Logo veremos que uma forma de abordar esse problema é tentar obter regularidade para a hierarquia projetiva, aumentando gradativamente a complexidade das classes projetivas. Paramos quando atingimos a força de consistência de um cardinal inacessível. Por exemplo, Shelah provou em 1984 [She84] que a Baire mensurabilidade para todos os conjuntos projetivos de reais é possível na força de consistência de ZFC, enquanto que a Lebesgue mensurabilidade para os conjuntos $\boldsymbol{\Sigma}_{3}^{1}$ implica na existência de um cardinal inacessível, quebrando de vez a aparente dualidade entre medida e categoria. Nos restringiremos somente à análise dos conjuntos $\Delta_{2}^{1}$ e $\Sigma_{2}^{1}$.

\subsection{O forcing aleatório foi o primeiro forcing idealizado}

Em 1964 [Sol70] (note que a publicação foi em 1970), apenas um ano após a introdução do forcing por Cohen, Solovay resolveu o Problema da Medida, mencionado na Seção 1.4, estabelecendo o seguinte resultado:

Teorema 4.2.1. Se existe um cardinal inacessivel, então

1. Existe um modelo de $\mathrm{ZFC}$ onde todo subconjunto projetivo dos reais é Lebesgue mensurável e Baire mensurável.

2. Existe um modelo de $\mathrm{ZF}+\mathrm{DC}$ onde todo subconjunto dos reais é Lebesgue mensurável e Baire mensurável.

Demonstração. Veja o Corolário 5.2.11 referente ao Teorema 5.2.10.

A analogia que existe entre medida e categoria permitiu com que Solovay fizesse praticamente a mesma prova tanto para a Lebesgue mensurabilidade, quanto para a Baire mensurabilidade. Em um argumento separado, Solovay também obteve a propriedade do conjunto perfeito.

Teorema 4.2.2 (Continuação do Teorema 4.2.1). 
1'. Para o mesmo modelo do Teorema 4.2.1, item 1., vale a propriedade do conjunto perfeito para todos os conjuntos projetivos.

2'. Para o mesmo modelo do Teorema 4.2.1, item 2., vale a propriedade do conjunto perfeito para todos os subconjuntos de reais.

Até a publicação de [Sol70], foram considerados apenas forcings cujas condições eram funções parciais. Baseado no fato de que os conjuntos mensuráveis podem ser aproximados, em um sentido preciso, por conjuntos borelianos, Solovay desenvolveu uma noção específica de forcing para atacar o Problema da Medida.

Definição 4.2.3. Considere $\mu$ a medida de Lebesgue em $\omega^{\omega}$.

1. Definimos $=_{\mathcal{N}}$ uma relação de equivalência em $\mathcal{B}\left(\omega^{\omega}\right)$ da seguinte forma: dados $A, B \in \mathcal{B}\left(\omega^{\omega}\right)$,

$$
A=\mathcal{N} B \leftrightarrow A \Delta B \in \mathcal{N} .
$$

2. Dado $A \in \mathcal{B}\left(\omega^{\omega}\right)$, a classe de $A$ é o conjunto

$$
[A] \doteq\left\{B \in \mathcal{B}\left(\omega^{\omega}\right) \mid A=_{\mathcal{N}} B\right\} .
$$

3. Denotaremos o quociente $\mathbb{B}\left(\omega^{\omega}\right) /=_{\mathcal{N}}$ simplesmente por $\mathcal{B}\left(\omega^{\omega}\right) / \mathcal{N}$.

Definição 4.2.4. Definimos o forcing aleatório, ou álgebra aleatória, como $\left(\mathbb{B}, \leq_{\mathcal{N}}, \mathbb{1}\right)$, onde

- $\mathbb{B}=\mathcal{B}\left(\omega^{\omega}\right) / \mathcal{N}$;

- $[A] \leq_{\mathcal{N}}[B] \leftrightarrow A \backslash B \in \mathcal{N}$;

- o maior elemento é $\mathbb{1}=\left[\omega^{\omega}\right]$

É possível mostrar que $\mathbb{B}$ é uma álgebra de Boole completa (e por isso o nome "álgebra aleatória"). É importante observar que a definição da ordem $\leq_{\mathcal{N}}$ não depende da escolha dos representantes da classe de equivalência e, além disso, podemos definir naturalmente uma medida em $\mathbb{B}$ a partir da medida de Lebesgue em $\omega^{\omega}$ : se $A$ é um conjunto mensurável, então

$$
\mu_{\mathbb{B}}([A]) \doteq \mu(A) .
$$

Novamente, a definição da medida acima não depende da escolha do representante para a classe de equivalência de $A$.

Essa álgebra de Boole completa é o completamento da noção de forcing definida por $\mathbb{P}_{\mathcal{N}} \doteq$ $\mathcal{B}\left(\omega^{\omega}\right) \backslash \mathcal{N}$, com a ordem de inclusão direta e maior elemento $\omega^{\omega}$. Portanto, forçar com $\mathbb{B}$ é equivalente a forçar com $\mathbb{P}_{\mathcal{N}}$, embora a álgebra de Boole tenha a vantagem de tornar os argumentos mais simples. Desenvolveremos a noção de regularidade para forcings "da forma $\mathbb{P}_{\mathcal{N}}$ ". Tornando essa noção precisa:

Definição 4.2.5. Seja $J$ um $\sigma$-ideal em $\omega^{\omega}$. Definimos o forcing idealizado $\left(\mathbb{P}_{J}, \leq, \mathbb{1}\right)$ onde

- $\mathbb{P}_{J}=\mathcal{B}\left(\omega^{\omega}\right) \backslash J$ é o conjunto dos borelianos $J$-positivos;

- a ordem $\leq$ é a inclusão direta $\subseteq$;

- o maior elemento é $\mathbb{1}=\omega^{\omega}$.

Teorema 4.2.6. O forcing $\mathbb{P}_{\mathcal{N}}$ tem ccc. 
Demonstração. Primeiramente note que duas condições distintas $B$ e $C$ são incompatíveis se, e somente se, $B \cap C$ é nulo.

Seja $E \subseteq \mathbb{P}_{\mathcal{N}}$ tal que $|E|=\aleph_{1}$. Vamos mostrar que $E$ não é anticadeia. Para cada $n<\omega$, considere $E_{n} \doteq\left\{B \in E \mid \mu(B)>\frac{1}{n+1}\right\}$ e note que $E=\bigcup_{n<\omega} E_{n}$. Como $|E|=\aleph_{1}$, então existe $n<\omega$ tal que $E_{n}$ é não-enumerável. Assim, existem $B, C \in E_{n}$ distintos tais que $\mu(B \cap C)>\frac{1}{n+1}$ e, portanto, $B$ e $C$ são compatíveis. Segue que $E$ não é anticadeia.

Seguindo a analogia que existe entre medida e categoria, podemos definir uma álgebra análoga, trocando o $\sigma$-ideal $\mathcal{N}$ dos conjuntos nulos pelo $\sigma$-ideal $\mathcal{M}$ dos conjuntos magros.

\section{Definição 4.2.7.}

1. Definimos $=\mathcal{M}$ uma relação de equivalência em $\mathcal{B}\left(\omega^{\omega}\right)$ da seguinte forma: dados $A, B \in \omega^{\omega}$,

$$
A=\mathcal{M} B \leftrightarrow A \Delta B \in \mathcal{M} .
$$

2. Dado $A \in \mathcal{B}\left(\omega^{\omega}\right)$, a classe de $A$ é o conjunto

$$
[A]=\left\{B \in \mathcal{B}\left(\omega^{\omega}\right) \mid A=\mathcal{M} B\right\} .
$$

3. Denotaremos o quociente $\mathcal{B}\left(\omega^{\omega}\right) /={ }_{\mathcal{M}}$ simplesmente por $\mathcal{B}\left(\omega^{\omega}\right) / \mathcal{M}$. Definimos o forcing $\left(\mathcal{B}\left(\omega^{\omega}\right) / \mathcal{M}, \leq_{\mathcal{M}}, \mathbb{1}\right)$, onde o maior elemento é $\mathbb{1}=\left[\omega^{\omega}\right]$ e

$$
[A] \leq_{\mathcal{M}}[B] \leftrightarrow A \backslash B \in \mathcal{M} .
$$

Novamente, o forcing $\mathcal{B}\left(\omega^{\omega}\right) / \mathcal{M}$ é o completamento do forcing $\mathbb{P}_{\mathcal{M}}$ (Definição 4.2.5). No próximo teorema, veremos que esse é equivalente ao forcing $\mathbb{C}$ de Cohen.

Teorema 4.2.8. O forcing $\mathbb{C}$ de Cohen é equivalente ao forcing $\mathcal{B}\left(\omega^{\omega}\right) / \mathcal{M}$.

Demonstração. Seja $B$ é um boreliano não magro. Como todo boreliano é Baire mensurável, existe um aberto não vazio $O$ tal que $B \Delta O \in \mathcal{M}$. Isto é, $O=\mathcal{M} B$. Por outro lado, existe $s \in \omega^{<\omega}$ tal que $\mathcal{O}_{s} \subseteq O$. Logo, $\mathcal{O}_{s} \backslash B \in \mathcal{M}$ e, portanto, $\left[\mathcal{O}_{s}\right] \leq_{\mathcal{M}}[B]$. Assim, o mapa $e: \mathbb{C} \rightarrow \mathcal{B}\left(\omega^{\omega}\right) / \mathcal{M}$ definido por $e(s)=\left[\mathcal{O}_{s}\right]$ é um mergulho denso, e portanto $\mathbb{C}$ e $\left(\mathcal{B}\left(\omega^{\omega}\right) / \mathcal{M} \leq_{\mathcal{M}}\right)$ são forcing-equivalentes.

Notação. Denotaremos o forcing $\left(\mathcal{B}\left(\omega^{\omega}\right) / \mathcal{M}, \leq \mathcal{M}, \mathbb{1}\right)$ por $\mathbb{C}$, por conta do Teorema 4.2.8.

Resumindo os comentários acima:

Teorema 4.2.9.

1. $\mathbb{P}_{\mathcal{N}}$ e $\mathbb{B}$ são forcing-equivalentes.

2. $\mathbb{P}_{\mathcal{M}} e \mathbb{C}$ são forcing-equivalentes.

Demonstração.

1. O completamento de $\mathbb{P}_{\mathcal{N}}$ é $\mathbb{B}$.

2. O completamento de $\mathbb{P}_{\mathcal{M}}$ é $\mathbb{C}$. 
Teorema 4.2.10. Seja $G$ um filtro $\mathbb{B}$-genérico. Então existe um único real $x_{G} \in V[G]$ tal que para qualquer boreliano $B$ codificado em $\mathrm{V}$

$$
x_{G} \in B^{\mathrm{V}[G]} \leftrightarrow B^{\mathrm{V}} \in G .
$$

Demonstração. Veja o Teorema 5.1.2.

\section{Definição 4.2.11.}

1. O número real $x_{G}$ do Teorema 4.2.10 é dito real aleatório.

2. Seja $M$ um modelo transitivo para ZFC. Dizemos um real $x$ é aleatório sobre $M$ se existe algum $G$ filtro $\mathbb{B}^{M}$-genérico sobre $M$ tal que para qualquer boreliano $B$ codificado em $M$ temos

$$
x \in B^{M[G]} \leftrightarrow B^{M} \in G .
$$

O nome aleatório foi dado por Solovay porque esse real pode ser caracterizado como o único real que não pertence a nenhum boreliano do modelo base. Ou seja, em algum sentido, a probabilidade de um número real arbitrário ser esse "real aleatório" é zero.

Teorema 4.2.12. Seja $M$ um modelo transitivo de ZFC. Então um real x é aleatório sobre $M$ se, e somente se, $x \notin B$ para qualquer boreliano nulo $B$ codificado em $M$.

Demonstração. Veja o Teorema 5.1.13.

Solovay também conseguiu caracterizar a Lebesgue mensurabilidade e a Baire mensurabilidade dos conjuntos $\boldsymbol{\Sigma}_{2}^{1}$ via forcing. Mais precisamente, Solovay mostrou que a Lebesgue mensurabilidade para os conjuntos $\boldsymbol{\Sigma}_{2}^{1}$ é equivalente a dizer que quase todos os reais são aleatórios sobre L[r], para todo real $r$. Analogamente, todos os conjuntos $\boldsymbol{\Sigma}_{2}^{1}$ são Baire mensuráveis se, e somente se, o conjunto dos reais de Cohen sobre $\mathrm{L}[r]$ é comagro, para todo real $r$.

\section{Teorema 4.2.13.}

1. Todos os conjuntos $\boldsymbol{\Sigma}_{2}^{1}$ de reais são Lebesgue mensuráveis se, e somente se, para todo real $r$

$$
\left\{x \in \omega^{\omega} \mid x \text { não é real aleatório sobre } \mathrm{L}[r]\right\} \in \mathcal{N} .
$$

2. Todos os conjuntos $\boldsymbol{\Sigma}_{2}^{1}$ de reais são Baire mensuráveis se, e somente se, para todo real $r$

$$
\left\{x \in \omega^{\omega} \mid x \text { não é real de Cohen sobre } \mathrm{L}[r]\right\} \in \mathcal{M} \text {. }
$$

Demonstração. Veja o Teorema 5.3.14.

Esse tipo de caracterização para a regularidade dos conjuntos $\boldsymbol{\Sigma}_{2}^{1}$ - i.e., a caracterização da regularidade para esses conjuntos via existência de algum conjunto "grande" de reais adicionados quando forçamos sobre L $[r]$ - é dita caracterização tipo Solovay.

Além desse tipo de caracterização, teremos também uma forma de caracterizar a regularidade para os conjuntos $\boldsymbol{\Delta}_{2}^{1}$ : caracterizações tipo Ihoda-Shelah. Ihoda e Shelah provaram em 1989 [IS89] que a Lebesgue mensurabilidade e a Baire mensurabilidade são equivalentes à existência de um real aleatório, respec. de Cohen, sobre L $[r]$, para todo real $r$.

Teorema 4.2.14 ([IS89]).

1. Todos os conjuntos $\boldsymbol{\Delta}_{2}^{1}$ de reais são Lebesgue mensuráveis se, e somente se, para todo real $r$, existe um real aleatório sobre $L[r]$. 
2. Todos os conjuntos $\boldsymbol{\Delta}_{2}^{1}$ de reais são Baire mensuráveis se, e somente se, para todo real $r$, existe um real de Cohen sobre $L[r]$.

Demonstração. Veja o Teorema 5.3.13.

A caracterização da propriedade do conjunto perfeito para conjuntos projetivos é diferente. A propriedade do conjunto perfeito já para o nível $\Pi_{1}^{1}$ implica na existência de um cardinal inacessível.

Teorema 4.2.15 ([Sol69]). São equivalentes:

1. Todos os conjuntos $\boldsymbol{\Sigma}_{2}^{1}$ de reais têm a propriedade do conjunto perfeito.

2. Todos os conjuntos $\Pi_{1}^{1}$ de reais têm a propriedade do conjunto perfeito.

3. $\forall r \in \omega^{\omega}\left(\aleph_{1}^{\mathrm{L}[r]}<\aleph_{1}\right)$.

\subsection{Marczewski mensurabilidade e o forcing de Sacks}

Marczewski em 1935 [Szp35] investigou uma classe de funções introduzida por Sierpiński: funções reais $f$ tais que para todo conjunto perfeito $P$, existe um conjunto perfeito $Q \subseteq P$ tal que $f\lceil Q$ é contínua. Nessa investigação, Marczewski introduziu uma noção de regularidade que hoje leva seu nome.

Definição 4.3.1. Um conjunto $A \subseteq \omega^{\omega}$ é dito Marczewski mensurável se para todo conjunto perfeito $P$ existe um conjunto perfeito $Q \subseteq P$ tal que $Q \subseteq A$ ou $Q \cap A=\emptyset$.

Pensando na ideia de que certos conjuntos de reais podem ser aproximados por árvores, como fizemos no Capítulo 2, introduziremos árvores adequadas para o nosso contexto, i.e., árvores que aproximam conjuntos de reais: as árvores reais. Além disso, são introduzidos alguns elementos arbóreos que serão úteis em outras seções.

Definição 4.3.2. Dizemos que $T$ é uma árvore real se é uma árvore como na Definição 2.2.3, onde $k=0$ e $X=\omega$ ou $X=2$. Para uma árvore real $T$, temos alguns conceitos especiais associados.

- Se $t \in T$, definimos o conjunto dos sucessores imediatos de $t$ por

$$
\operatorname{Succ}_{T}(t)=\left\{s \in T \mid \exists n<\omega\left(t^{\frown} n=s\right)\right\}
$$

- Um nó $t \in T$ ramifica se $\left|\operatorname{Succ}_{T}(t)\right|>1$. O conjunto de todos os nós de $T$ que ramificam é denotado por $\operatorname{Split}(T)$.

- Para cada $s \in \operatorname{Split}(T)$, definimos

$$
\begin{aligned}
\operatorname{Split}(\overleftarrow{s}) & =\{t \in \operatorname{Split}(T) \mid t \subseteq s\} \text { e, para cada } n<\omega \\
\operatorname{Split}_{n}(T) & =\{s \in \operatorname{Split}(T)|| \operatorname{Split}(\overleftarrow{s}) \mid=n\} .
\end{aligned}
$$

- O conjunto de todos os niveis ramificantes de T é definido por

$$
\operatorname{Splev}(\mathrm{T})=\{|t| \mid t \in \operatorname{split}(T)\} \text {. }
$$

- Um nó $t \in T$ é dito terminal se $\operatorname{Succ}_{T}(t)=\emptyset$.

- Se $s \in T$, então a subárvore de T restrita a s é definida por

$$
T \nmid s \doteq\{t \in T \mid t \subseteq s \vee s \subseteq t\}
$$


- O tronco de $T$, que denotamos por $\operatorname{stem}(T)$, quando existe, é o nó maximal s tal que $T\lceil s=$ $T$.

Agora, todo conjunto perfeito é fechado. Se $P \subseteq \omega^{\omega}$ é um conjunto perfeito, pelo Lema 2.2.5, existe uma árvore $T$ tal que $[T]=P$, e diremos que uma árvore assim é uma árvore perfeita. É possível provar que a seguinte definição de árvore perfeita recupera exatamente a definição de conjunto perfeito.

Definição 4.3.3. Seja $T$ uma árvore real. Dizemos que $T$ é uma árvore perfeita se para todo $t \in T$, existe $s \in \operatorname{Split}(T)$ que estende $t$.

Lema 4.3.4. Se $T$ é uma árvore perfeita então $[T]$ é um conjunto perfeito. Reciprocamente, se $P$ é um conjunto perfeito, então existe $T$ uma árvore perfeita tal que $P=[T]$.

Agora, podemos reformular a propriedade do conjunto perfeito e a Marczewski mensurabilidade usando árvores perfeitas.

Corolário 4.3.5. Seja $A \subseteq \omega^{\omega}$ um conjunto.

1. A tem a propriedade do conjunto perfeito se, e somente se, A é enumerável ou existe $T$ uma árvore perfeita tal que $[T] \subseteq A$.

2. A é Marczewski mensurável se, e somente se, para toda árvore perfeita $T$, existe uma árvore perfeita $S \subseteq T$ tal que $[S] \subseteq A$ ou $[S] \cap A=\emptyset$.

A partir disso, podemos considerar o forcing com árvores perfeitas, também conhecido como forcing de Sacks.

Definição 4.3.6. O forcing de Sacks é o forcing $(\mathbb{S}, \leq, \mathbb{1})$, onde

- as condições em $\mathbb{S}$ são árvores perfeitas;

- a ordem $\leq e ́$ a inclusão direta $\subseteq$;

- o maior elemento é $\mathbb{1}=\omega^{<\omega}$.

Forcings com árvores reais ordenadas por inclusão são ditos forcings arbóreos. Esses tipos de forcing constituem uma classe importante de forcings, e todos os exemplos de forcings estudados nesse texto são equivalentes a forcings arbóreos (Veja [Ike10]). Nosso interesse, porém, é colocar os forcings arbóreos na forma idealizada.

O forcing de Sacks é um forcing próprio que adiciona um número real, conhecido como real de Sacks.

Definição 4.3.7. Seja $G$ um filtro $\mathbb{S}$-genérico. Então o número real $f \doteq \bigcup G$ é dito real de Sacks. $\dashv$

Embora o contexto da Marczewski mensurabilidade seja natural para introduzir o forcing de Sacks, Sacks em 1971 [Sac71] introduziu o forcing com árvores perfeitas para obter um modelo com grau mínimo de construtibilidade. Um filtro genérico $G$ tem grau mínimo de construtibilidade se para todo conjunto de ordinais $A \in V[G]$, ou $A \in V$ ou $G \in V[X]$, i.e., ou o conjunto de ordinais já está no modelo base, ou $V[G]=V[A]$. Agora, se $f$ é um real de Sacks, então $V[f]=V[G]$, pois o filtro $G$ é definível a partir de $f: G=\{T \in \mathbb{S} \mid \forall n<\omega(f\lceil n \in T)\}$. Por conta disso, dizemos que o real de Sacks é um real com grau mínimo de construtibilidade.

A fim de colocar o forcing $\mathbb{S}$ na forma idealizada, vamos construir um mergulho denso de $\mathbb{S}$ em algum $\mathbb{P}_{J}$. A propriedade do conjunto perfeito nos dá uma pista: todo boreliano tem a propriedade do conjunto perfeito (Teorema 1.4.12). Assim, um boreliano ou é enumerável, ou contém o conjunto dos ramos de uma árvore perfeita. Esse fato nos permite construir um mergulho denso de $\mathbb{S}$ em $\mathbb{P}_{\text {ctbl }}$. Provando o Teorema de Davis (Teorema 2.4.11) e então o Teorema 1.4.12 seguirá do Teorema da Determinância Boreliana. A vantagem dessa prova é que ela pode ser adaptada para outras noções noções de regularidade, como por exemplo as que serão introduzidas nas duas próximas seções. 
Teorema (Veja 2.4.11). Seja $A \subseteq \omega^{\omega}$.

1. Se o jogador I tem uma estratégia vencedora em $G^{*}(A)$, então A possui um subconjunto não vazio perfeito.

2. Se o jogador II tem uma estratégia vencedora em $G^{*}(A)$, então A é enumerável.

\section{Demonstração.}

1. Sejam $\sigma$ uma estratégia vencedora para I,

$$
\operatorname{Plays}(\sigma) \doteq\left\{\sigma * y \mid y \in \omega^{\omega}\right\}
$$

todas os possíveis jogadas usando a estratégia $\sigma$ e

$$
T_{\sigma} \doteq\left\{s \in \omega^{<\omega} \mid \exists t \in \omega^{<\omega}(s \subseteq \sigma * t)\right\}
$$

Note que $T_{\sigma}$ é uma árvore e $\left[T_{\sigma}\right]=\operatorname{Plays}(\sigma) \subseteq A$. Além disso, $T_{\sigma}$ é uma árvore perfeita.

De fato, seja $t \in T_{\sigma}$ e considere o menor $i$ tal que $t \subseteq \widehat{s_{0}} \widehat{s}_{1} \ldots{ }^{\wedge} s_{i}$. Se o jogador II joga $n_{i+1}$, a estratégia $\sigma$ permite ao jogador I escolher $s_{i+1}$ de modo que $s_{i+1}(0) \neq n_{i+1}$, e se o jogador II joga $m_{i+1} \neq n_{i+1}$, o jogador I escolhe $t_{i+1}$ de modo que $t_{i+1}(0) \neq m_{i+1}$. Claramente $\left.\widehat{s_{0}} \widehat{s_{1} \ldots}\right\urcorner s_{i} s_{i+1}$ e $\left.\widehat{s_{0}} \widehat{s_{1}} \ldots\right\urcorner s_{i} t_{i+1}$ são extensões incompatíveis de $t$, de acordo com $\sigma$, e assim são elementos de $T_{\sigma}$.

2. Seja $\tau$ uma estratégia para II e considere $p=\left(s_{0}, n_{1}, s_{1}, \ldots, s_{i-1}, n_{i}\right)$ uma jogada parcial em que II joga sob $\tau$, e é a vez de I jogar. Denotemos o conjunto de todos os $p$ 's como descritos anteriormente por $P_{\tau, I}$. Seja $\left.p^{*} \doteq s_{0} \widehat{s_{1}} \ldots\right\urcorner s_{i-1}$. Para cada $p \in P_{\tau, I}$ e $x \in \omega^{\omega}$, dizemos que:

- $p$ é compatível com $x$ se existe $s_{i}$ tal que $s_{i}(0) \neq n_{i}$ e $p^{* \frown} s_{i} \subseteq x$. Observe que isso ocorre somente se $p^{*} \subseteq x$ e $n_{i} \neq x(j)$, para todo $j<\omega$. Intuitivamente isso quer dizer que na posição $p$, o jogador I ainda tem uma chance de formar a sequência $x$.

- $p$ rejeita $x$ se $p$ é compatível com $x$ e é maximal com essa propriedade, i.e.: se $s_{i}$ é tal que $s_{i}(0) \neq n_{i}$ e $p^{* \frown} s_{i} \subseteq x$, quando o jogador II jogar $\tau\left(p^{\frown} s_{i}\right) \doteq n_{i+1}$, então $q \doteq p^{\frown}\left(s_{i}, n_{i+1}\right)$ não é mais compatível com $x$. Intuitivamente, isso significa que o jogador I tem a chance de continuar a estender suas jogadas na direção de $x$, mas só por mais um movimento, e na próxima jogada o jogador II vai estragar seu jogo, usando a estratégia $\tau$.

Afirmação 1: Para todo $x \in A$, existe $p \in P_{\tau, I}$ que rejeita $x$.

Demonstração. Suponha que não. Seja $x$ tal que nenhum $p$ rejeita $x$. Isso significa que em cada estágio do jogo, o jogador I pode jogar $s_{i}$ tal que $s_{0}^{\frown} s_{1}^{\frown} \ldots \frown s_{i} \subseteq x$ e o jogador II com a estratégia $\tau$ não pode fazer nada para impedir. Assim, existe uma sequência $y$ jogada por I tal que $y * \tau=x \in A$, o que contradiz que $\tau$ é uma estratégia vencedora.

Afirmação 2: Cada $p \in P_{\tau, I}$ rejeita no máximo um $x$.

Demonstração. Suponha que $p=\left(s_{0}, n_{1}, s_{1}, \ldots, s_{i-1}, n_{i}\right)$ é compatível com $x$ e $y$, e $x \neq y$. Considere então $s_{i}$ maximal tal que $s_{i}(0) \neq n_{i}$ e $p^{* \frown} s_{i} \subseteq x, y$ (isso é possível pois $x(n) \neq y(n)$ para algum $n<\omega)$. Assim $n_{i+1} \doteq \tau\left(p^{\frown} s_{i}\right)$ não pode aparecer em ambos $x$ e $y, \operatorname{logo} p^{\curvearrowleft}\left(s_{i}, n_{i+1}\right)$ ainda pode ser estendido pelo jogador I para ser compatível com $x$ ou $y$. Segue que $p$ não pode rejeitar ambos $x$ e $y$.

Finalmente, o mapa $x \mapsto p_{x}$ que leva $x \in A$ no único $p_{x} \in P_{\tau, I} \subseteq\left(\omega^{<\omega}\right)^{<\omega}$ que rejeita $x$ é uma injeção. Segue que $A$ é enumerável. 
Corolário 4.3.8. $\mathbb{P}_{\text {ctbl }}$ e $\mathbb{S}$ são forcing-equivalentes.

Demonstração. O mapa $T \mapsto[T]$ é um mergulho denso de $\mathbb{S}$ em $\mathbb{P}_{\text {ctbl }}$.

\subsection{E se trocarmos árvores perfeitas por superperfeitas?}

Nessa seção investigaremos uma nova noção de grandeza para os reais (na verdade, essa noção só faz sentido para o espaço de Baire). Na propriedade do conjunto perfeito, os conjuntos pequenos são os conjuntos enumeráveis, e os conjuntos grandes são os conjuntos perfeitos. Agora, os conjuntos pequenos serão os conjuntos $\sigma$-limitados, e os conjuntos grandes serão os conjuntos que contém subconjuntos superperfeitos. Em resumo:

$$
\frac{\text { enumerável }}{\sigma \text {-limitado }}=\frac{\text { perfeito }}{\text { superperfeito }} .
$$

Portanto, assim como na propriedade do conjunto perfeito, temos uma dicotomia: um conjunto ou é $\sigma$-limitado ou contém um subconjunto superperfeito. Essa dicotomia foi isolada por Kechris em 1977 [Kec77], entretando a noção de conjunto superperfeito já havia aparecido implicitamente em 1973 [Fri73], de onde vem a motivação para essa dicotomia.

Antes de definir os conceitos-chave, precisamos definir duas relações de dominância em $\omega^{\omega}$

Definição 4.4.1. Sejam $x, y \in \omega^{\omega}$. As relações $\leq e \leq^{*} e m \omega^{\omega}$ são definidas da seguinte forma:

1. $x \leq y \leftrightarrow \forall n<\omega(x(n) \leq y(n))$.

2. $x \leq^{*} y \leftrightarrow \forall^{\infty} n<\omega(x(n) \leq y(n))$. Nesse caso, dizemos que $y$ domina $x$.

Teorema 4.4.2. Seja $A \subseteq \omega^{\omega}$. São equivalentes:

1. Existe uma sequência $\left\{x_{i} \mid i<\omega\right\}$ em $\omega^{\omega}$ tal que, para todo $a \in A$, existe $i<\omega$ tal que $a \leq x_{i}$

2. A está contido em um conjunto $\sigma$-compacto de $\omega^{\omega}$.

3. Existe $x \in \omega^{\omega}$ tal que $a \leq^{*} x$, para todo $a \in A$.

\section{Definição 4.4.3.}

1. Um conjunto $A \subseteq \omega^{\omega}$ é dito $\sigma$-limitado se satisfaz algum dos itens de 1. a 3. do Teorema 4.4.2.

2. $K_{\sigma}$ é o $\sigma$-ideal dos conjuntos $\sigma$-limitados.

Agora, os nossos conjuntos grandes, i.e., superperfeitos, são obtidos fortalecendo o conceito de árvore perfeita.

Definição 4.4.4. Uma árvore $T$ em $\omega$ é dita superperfeita se todo nó $t \in T$ tem uma extensão $s \supseteq t$ que é infinitamente ramificada. Isto é, $\left|\operatorname{Succ}_{T}(s)\right|=\omega$. Um conjunto $A \subseteq \omega^{\omega}$ é superperfeito se existe $T$ uma árvore superperfeita tal que $[T]=A$.

Assim, obtemos uma dicotomia análoga à propriedade do conjunto perfeito, que chamaremos de $K_{\sigma}$-dicotomia, e uma noção de regularidade análoga à Marczewski mensurabilidade, que chamaremos de Miller mensurabilidade. A noção de Miller mensurabilidade é obtida trocando árvores perfeitas por superperfeitas na definição de Marczewski mensurabilidade. Como vimos, as árvores perfeitas são condições do forcing de Sacks, e como Miller foi a primeira pessoa a considerar um forcing com árvores superperfeitas, essa noção de regularidade hoje leva o seu nome. 
Definição 4.4.5. Um conjunto $A \subseteq \omega^{\omega}$ satisfaz a dicotomia $K_{\sigma}$ se $A$ é $\sigma$-limitado ou se existe uma árvore superperfeita $T$ tal que $[T] \subseteq A$.

Definição 4.4.6. Um conjunto $A \subseteq \omega^{\omega}$ é dito Miller mensurável se para toda árvore superperfeita $T$, existe uma árvore superperfeita $S \subseteq T$ tal que $[S] \subseteq A$ ou $[S] \cap A=\emptyset$.

Definição 4.4.7. O forcing de Miller é o forcing $(\mathbb{M}, \leq, \mathbb{1})$, onde

- as condições em $\mathbb{M}$ são árvores superperfeitas,

- $a$ ordem $\leq$ é a inclusão direta $\subseteq$, e

- o maior elemento é $\mathbb{1}=\omega^{<\omega}$.

O forcing de Miller é um forcing próprio que adiciona um número real, conhecido como real de Miller.

Definição 4.4.8. Seja $G$ um filtro $\mathbb{M}$-genérico. Então o número real $f \doteq \bigcup G$ é dito real de Miller.

O forcing de Miller foi introduzido por Miller em 1984 [Mil84] sob o nome de forcing perfeito racional. Miller estava interessado em estudar conjuntos perfeitos que contêm densamente os racionais. Miller logo percebeu que esses conjuntos são os conjuntos da forma $[T]$, onde $T$ é uma árvore superperfeita.

Assim como no caso do forcing de Sacks, o forcing de Miller admite uma forma idealizada. Não é surpreendente que o forcing idealizado correspondente ao forcing de Miller seja o forcing $\mathbb{P}_{K_{\sigma}}$. Novamente, construiremos um mergulho denso de $\mathbb{M}$ em $\mathbb{P}_{K_{\sigma}}$, e para isso vamos provar a dicotomia $K_{\sigma}$ para os borelianos. Em analogia com o jogo de Davis, Kechris em [Kec77] introduziu o jogo $\widetilde{G}$.

Definição 4.4.9. Seja $A \subseteq \omega^{\omega}$. O jogo de Kechris $\widetilde{G}(A)$ é definido da seguinte forma:

- O jogador I joga com sequências finitas não vazias de números naturais (i.e., elementos de $\left.\omega^{<\omega}\right)$. O jogador II joga com números naturais, como ilustrado abaixo.

$\begin{array}{lllllll}I & s_{0} & & s_{1} & & \cdots & \\ I I & & n_{1} & & n_{2} & & \cdots\end{array}$

- O jogador I ganha o jogo $\widetilde{G}(A)$ se, e somente se

(a) $\forall i \geq 1\left(s_{i}(0)>n_{i}\right) e$

(b) $x \doteq s_{0} s_{1}^{\frown} s_{2}^{\imath} \cdots \in A$.

Teorema 4.4.10 ([Kec77]). Seja $A \subseteq \omega^{\omega}$.

1. Se o jogador I tem uma estratégia vencedora em $\widetilde{G}(A)$, então A possui um subconjunto não vazio superperfeito.

2. Se o jogador II tem uma estratégia vencedora em $\widetilde{G}(A)$, então A é $\sigma$-limitado.

Como consequência, vale a dicotomia $K_{\sigma}$ para os borelianos.

Demonstração. A demonstração é análoga à do Teorema 2.4.11. 
1. Sejam $\sigma$ uma estratégia vencedora para I,

$$
\operatorname{Plays}(\sigma) \doteq\left\{\sigma * y \mid y \in \omega^{\omega}\right\}
$$

todas os possíveis jogadas usando a estratégia $\sigma$ e

$$
T_{\sigma} \doteq\left\{s \in \omega^{<\omega} \mid \exists t \in \omega^{<\omega}(s \subseteq \sigma * t)\right\} .
$$

Note que $T_{\sigma}$ é uma árvore e $\left[T_{\sigma}\right]=\operatorname{Plays}(\sigma) \subseteq A$. De forma análoga à demonstração do Teorema 2.4.11 obtemos que $T_{\sigma}$ é uma árvore superperfeita.

De fato, seja $t \in T_{\sigma}$ e considere o menor $i$ tal que $\left.t \subseteq s_{0} \widehat{s_{1}} \ldots\right\urcorner s_{i}$. Para qualquer $n_{i+1}$ que o jogador II escolher, o jogador I seguindo a estratégia $\sigma$ pode escolher $s_{i+1}$ de modo que $s_{i+1}(0)>n_{i+1}$, obtendo então uma infinidade de $s_{i+1}$ 's com $s_{i+1}(0)$ cada vez maiores, e para esses infinitos $s_{i+1}$ 's, $\left.s_{0} s_{1}^{\frown} \ldots\right\urcorner s_{i} s_{i+1} \in T_{\sigma}$.

2. Exatamente como na demonstração do Teorema 2.4.11, seja $\tau$ uma estratégia para II e considere $p=\left(s_{0}, n_{1}, s_{1}, \ldots, s_{i-1}, n_{i}\right)$ uma jogada parcial em que II joga sob $\tau$, e é a vez de I jogar. Denotemos o conjunto de todos os $p$ 's como descritos anteriormente por $P_{\tau, I}$. Seja $p^{*} \doteq \widehat{s_{0}} \widehat{s_{1} \ldots} s_{i-1}$. Para cada $p \in P_{\tau, I}$ e $x \in \omega^{\omega}$, dizemos que:

- $p$ é compatível com $x$ se existe $s_{i}$ tal que $s_{i}(0)>n_{i}$ e $p^{* \frown} s_{i} \subseteq x$.

- $p$ rejeita $x$ se $p$ é compatível com $x$ e maximal com essa propriedade, i.e., se para todo $s_{i}$ tal que $s_{i}(0)>n_{i}$, temos que $p^{\curvearrowleft}\left(s_{i}, \tau\left(p^{\wedge} s_{i}\right)\right)$ não é compatível com $x$.

Afirmação 1: Para todo $x \in A$, existe $p \in P_{\tau, I}$ que rejeita $x$.

Demonstração. Veja a Afirmação 1 na demonstração do Teorema 2.4.11.

Afirmação 2: Para cada $p \in P_{\tau, I}$ o conjunto

$$
K_{p} \doteq\left\{x \in \omega^{\omega} \mid p \text { rejeita } x\right\}
$$

é $\sigma$-compacto.

Demonstração. Basta reescrever $K_{p}$ como

$$
\begin{aligned}
K_{p}=\left\{x \in \omega^{\omega}\right. & \mid \exists s_{i}\left[\left(s_{i}(0)>n_{i} \wedge p^{* \frown} s_{i} \subseteq x\right)\right. \\
\wedge & \left.\left.\forall s_{j}\left(\left(p^{\frown}\left(s_{i}, \tau\left(p^{\frown} s_{i}\right)\right)\right)^{*} s_{j} \subseteq x \rightarrow s_{j}(0) \leq \tau\left(p^{\frown} s_{i}\right)\right)\right]\right\}
\end{aligned}
$$

Agora, fixe $s_{i}$ tal que $s_{i}(0)<n_{i}$ e $p^{* \frown} s_{i} \subseteq x$, e $s_{j} \in \omega^{<\omega}$ qualquer. Defina

- $\left.K_{p, s_{i}, s_{j}} \doteq\left\{x \in \omega^{\omega} \mid\left(p^{\frown}\left(s_{i}, \tau\left(p^{\frown} s_{i}\right)\right)\right)^{*^{\frown}} s_{j} \subseteq x \rightarrow s_{j}(0) \leq \tau\left(p^{\frown} s_{i}\right)\right)\right\}$. Note que esses conjuntos são compactos.

- $K_{p, s_{i}} \doteq \bigcap_{s_{j} \in \omega<\omega} K_{p, s_{i}, s_{j}}$. Como o espaço de Baire é Hausdorff, cada um desses conjuntos também é compacto.

Agora note que

$$
K_{p}=\bigcup_{s_{i} \in \omega<\omega} K_{p, s_{i}}
$$

Portanto, cada $K_{p}$ é união enumerável de compactos. 
Finalmente, $A \subseteq \bigcup_{p \in P_{\tau, I}} K_{p}$. Segue que $A$ é $\sigma$-limitado.

Corolário 4.4.11. $\mathbb{P}_{K_{\sigma}}$ e $\mathbb{M}$ são forcing-equivalentes.

\subsection{Uma noção de regularidade advinda da conjectura de Borel}

Em 1919 [Bor19], em uma investigação sistemática acerca dos conjuntos de medida nula, Borel introduziu a seguinte generalização: um conjunto $A \subseteq \mathbb{R}$ (os reais de verdade) tem medida fortemente nula se para toda sequência $\left(\varepsilon_{n}\right)_{n<\omega}$ de reais positivos, existe uma sequência $\left(I_{n}\right)_{n<\omega}$ de intervalos abertos tais que $A \subseteq \bigcup_{n<\omega} I_{n}$ e $\operatorname{diam}\left(I_{n}\right)<\varepsilon_{n}$. Também diremos que $A$ é fortemente nulo. Consequências simples dessa definição são:

- Todo conjunto fortemente nulo é nulo.

- Os conjuntos fortemente nulos formam um $\sigma$-ideal, usualmente denotado por $\mathcal{S N}$.

- Todo conjunto enumerável é fortemente nulo.

Naturalmente, podemos nos perguntar se existe algum conjunto nulo que não é fortemente nulo. Pelos comentários acima, o candidato a contra-exemplo deve ser um conjunto nulo não enumerável. De fato, o conjunto de Cantor é um conjunto nulo que não é fortemente nulo. Para ver isso, é preciso provar que $\mathcal{S N}$ é invariante por funções contínuas e a função de Cantor é uma função contínua cuja imagem é o intervalo $[0,1]$. Agora, uma pergunta natural é

Existe algum conjunto não enumerável fortemente nulo?

Borel conjecturou que a resposta para essa pergunta é negativa, e essa afirmação é conhecida como Conjectura de Borel. A primeira resposta na direção de resolver esse problema foi dada por Sierpiński em 1928 [Sie28], construindo um conjunto não enumerável fortemente nulo usando CH. Somente em 1976 [Lav76] Laver, usando a técnica de forcing que hoje leva seu nome, resolveu completamente essa conjectura, construindo um modelo de ZFC onde $2^{\aleph_{0}}=\aleph_{2}$ e todo conjunto fortemente nulo é enumerável (implicando que a Conjectura de Borel é independente de ZFC).

A seguir, introduziremos a noção de forcing utilizada por Laver e faremos uma breve descrição de suas ferramentas.

Definição 4.5.1. Uma árvore $T$ em $\omega$ é dita árvore de Laver se todo nó $t \in T$ tal que $\operatorname{stem}(T) \subseteq t$ possui uma extensão $s \supseteq t$ infinitamente ramificada - isto é, $\left|\operatorname{Succ}_{T}(s)\right|=\omega$. Um conjunto $A \subseteq \omega^{\omega}$ é de Laver se existe uma árvore de Laver $T$ tal que $[T]=A$.

Definição 4.5.2. O forcing de Laver é o forcing $(\mathbb{L}, \leq, \mathbb{1})$, onde

- as condições em $\mathbb{L}$ são árvores de Laver,

- $a$ ordem $\leq$ é a inclusão direta $\subseteq, e$

- o maior elemento é $\mathbb{1}=\omega^{<\omega}$.

O forcing de Laver é um forcing próprio que adiciona um número real, conhecido como real de Laver.

Definição 4.5.3. Seja $G$ um filtro $\mathbb{L}$-genérico. Então o número real $f \doteq \bigcup\{\operatorname{stem}(T) \mid T \in G\}$ é dito real de Laver.

Laver provou um lema central para construção de seu modelo: 
Todo conjunto em $V$ fortemente nulo em $V[G]$ é enumerável em $V[G]$.

Por fim, Laver mostrou que a conjectura de Borel vale iterando esse forcing $\omega_{2}$ vezes com suporte enumerável, partindo de um modelo para GCH. Para mais detalhes sobre a conjectura de Borel, além do trabalho original [Lav76], veja também [Jec03] ou [BJ].

Agora, como o forcing de Laver é arbóreo, em analogia com os forcings de Sacks e Miller, podemos introduzir a Laver mensurabilidade.

Definição 4.5.4. Um conjunto $A \subseteq \omega^{\omega}$ é dito Laver mensurável se para toda árvore de Laver $T$, existe uma árvore de Laver $S \subseteq T$ tal que $[S] \subseteq A$ ou $[S] \cap A=\emptyset$.

O forcing de Laver foi estudado sob o ponto de vista da regularidade primeiramente em 1995 [GRSS95], com a intenção de comparar invariantes cardinais associados a ideias dos forcings de Miller e Laver.

Nosso objetivo agora é encontrar a forma idealizada do forcing de Laver. Nos casos dos forcings de Sacks e de Miller, construímos mergulhos densos $\mathbb{S} \hookrightarrow \mathbb{P}_{\text {ctbl }}$ e $\mathbb{M} \hookrightarrow \mathbb{P}_{K_{\sigma}}$, e para isso tivemos que provar que os borelianos satisfazem a propriedade do conjunto perfeito e a dicotomia $K_{\sigma}$. $\mathrm{O}$ mesmo deve ser feito para o forcing de Laver: a fim de construir um mergulho denso $\mathbb{L} \hookrightarrow \mathbb{P}_{\mathcal{L}^{0}}$, para algum $\sigma$-ideal $\mathcal{L}^{0}$, precisamos isolar uma noção adequada de grandeza (os conjuntos pequenos formarão o $\sigma$-ideal $\mathcal{L}^{0}$ ), que deve sugerir uma dicotomia natural. Finalmente, devemos provar que essa dicotomia é verdadeira para os borelianos. $\mathrm{O} \sigma$-ideal $\mathcal{L}^{0}$ é o ideal dos conjuntos não fortemente dominantes. Introduziremos esses conceitos a seguir.

\section{Definição 4.5.5.}

1. Sejam $x, f \in \omega^{\omega}$. Dizemos que $x$ domina fortemente $f$ se

$$
\forall^{\infty} n(x(n+1)>f(x(n))) .
$$

2. Um conjunto $A \subseteq \omega^{\omega}$ é dito fortemente dominante se para todo $f \in \omega^{\omega}$ existe $x \in A$ tal que $x$ domina fortemente $f$.

Definição 4.5.6. $\mathcal{L}^{0}$ é o $\sigma$-ideal gerado pelos conjuntos não fortemente dominantes.

Agora, podemos introduzir uma dicotomia associada a essa noção de grandeza.

Definição 4.5.7. Um conjunto $A \subseteq \omega^{\omega}$ é $\ell$-regular se não é fortemente dominante ou se existe uma árvore de Laver $T$ tal que $[T] \subseteq A$.

Da mesma forma que Kechris usou um argumento via jogos para verificar a dicotomia $K_{\sigma}$ para algumas classes de conjuntos (e.g., para os borelianos), no artigo [GRSS95] também foi desenvolvido um jogo associado à $\ell$-regularidade.

Definição 4.5.8. Seja $A \subseteq \omega^{\omega}$. O jogo de dominância $D(A)$ é definido da seguinte forma:

- O jogador I apenas na primeira jogada joga com uma sequência finita de números naturais (i.e., elementos de $\omega^{<\omega}$ ), e nas rodadas seguintes ele joga somente com números naturais. $O$ jogador II joga somente com números naturais, como ilustrado abaixo.

$\begin{array}{llllllll}I & s & & m_{0} & & m_{1} & & \cdots \\ I I & & n_{0} & & n_{1} & & \ldots & \end{array}$


(a) $\forall i \geq 1\left(m_{i}>n_{i}\right) e$

(b) $x \doteq s^{\wedge} m_{0}^{\imath} m_{1}^{\imath} \cdots \in A$.

Teorema 4.5.9 ([GRSS95]). Seja $A \subseteq \omega^{\omega}$.

1. Se o jogador I tem uma estratégia vencedora em $D(A)$, então $A$ possui um subconjunto de Laver não vazio.

2. Se o jogador II tem uma estratégia vencedora em $D(A)$, então A não é fortemente dominante.

Consequentemente, todo boreliano é $\ell$-regular.

Demonstração.

1. Sejam $\sigma$ uma estratégia vencedora para I,

$$
\operatorname{Plays}(\sigma) \doteq\left\{\sigma * y \mid y \in \omega^{\omega}\right\}
$$

todas os possíveis jogadas usando a estratégia $\sigma$ e

$$
T_{\sigma} \doteq\left\{s \in \omega^{<\omega} \mid \exists t \in \omega^{<\omega}(s \subseteq \sigma * t)\right\} .
$$

Note que $T_{\sigma}$ é uma árvore e $\left[T_{\sigma}\right]=\operatorname{Plays}(\sigma)$. De forma análoga às demonstrações dos Teoremas 2.4.11 e 4.4.10 obtemos que $T_{\sigma}$ é uma árvore de Laver.

2. Vamos mostrar que se $A$ é fortemente dominante, então II não tem uma estratégia vencedora em $D(A)$. Isto é, para qualquer estratégia $\tau$ de II, existe uma jogada $\left(s, m_{0}, m_{1}, \ldots, m_{k}, \ldots\right)$ em que I ganha. Assim, suponha $A$ fortemente dominante e seja $\tau$ uma estratégia para II. Defina funções $F: \omega^{<\omega} \times \omega \rightarrow \omega$ e $f \in \omega^{\omega}$ da seguinte forma:

- $F\left(s^{\frown} m_{0}^{\frown} \ldots{ }^{\frown} m_{k},|s|\right)=\tau\left(s, m_{0}, \ldots, m_{k}\right)$.

- $f(m)=\max \left\{F(s, i) \mid i \leq m \wedge s \in m^{\leq m+1}\right\}+m$.

Note que $f$ como acima é crescente, e então $f(m) \leq m$, para todo $m<\omega$. Além disso, como $A$ é fortemente dominante, existe $x$ que domina fortemente $f$, i.e., tal que

$$
\forall^{\infty} k x(k)>f(x(k-1)) .
$$

Assim, $x$ é crescente. Assim, tomando $m \doteq x(k-1)$, temos que $\forall^{\infty} k, m \leq k-1$ e, então, $x \uparrow k \in m+1^{m+1}$. Pela definição de $f$ temos que $f(m) \leq F(x \uparrow k, i)$, para todo $i \leq k$. Portanto,

$$
x(k)>f(x(k-1)) \geq F(x\lceil k, i) .
$$

Com isso, conseguimos construir uma jogada $\left(s, m_{0}, \ldots, m_{k}, \ldots\right)$ em que I ganha:

$$
\begin{array}{cc}
\text { I } & \text { II } \\
s=x \uparrow k_{0} & n_{0} \doteq \tau(s)=F\left(x \uparrow k_{0}, k_{0}\right) \\
m_{0} \doteq x\left(k_{0}+1\right) & \\
m_{1} \doteq x\left(k_{0}+2\right) & n_{1} \doteq \tau\left(s, m_{0}\right)=F\left(x \uparrow\left(k_{0}+1\right), k_{0}\right)
\end{array}
$$


Corolário 4.5.10. $\mathbb{P}_{\mathcal{L}^{0}}$ e $\mathbb{L}$ são forcing-equivalentes.

\subsection{Uma noção de regularidade relacionada com colorações de sub- conjuntos de $\omega$}

Ramsey em 1930 [Ram30] provou o teorema que hoje conhecemos como Teorema Finito de Ramsey para resolver um problema de lógica formal: a decidibilidade das classes de fórmulas $\exists \forall$ com identidade. Ramsey também estabeleceu uma versão infinita de seu teorema, que é basicamente o Princípio da Casa dos Pombos para subconjuntos de $\omega$ de cardinalidade finita.

Teorema 4.6.1 (Teorema Infinito de Ramsey [Ram30]). Sejam $n<\omega$ e c : $[\omega]^{n} \rightarrow 2$ uma bicoloração. Então existe um conjunto $H \in[\omega]^{\omega}$ que é c-monocromático. Equivalentemente, dada uma partição de $[\omega]^{n}$ em $A$ e $[\omega]^{n} \backslash A$, existe um conjunto $H \in[\omega]^{\omega}$ tal que $[H]^{n} \subseteq A$ ou $[H]^{n} \cap A=\emptyset$.

O Princípio da Casa dos Pombos é o teorema acima para o caso $n=1$. É fácil ver a equivalência entre colorações e partições: toda coloração $c:[\omega]^{n} \rightarrow 2$ induz uma partição de $[\omega]^{n}$ dada por $A \doteq c^{-1}(\{1\})$ e $\left[\omega^{n}\right] \backslash A \doteq c^{-1}(\{0\})$. Reciprocamente, qualquer partição $A$ trivialmente induz uma bicoloração.

É natural perguntar se é possível generalizar o Teorema Infinito de Ramsey para subconjuntos enumeráveis de $\omega$. Isto é:

Para toda coloração $c:[\omega]^{\omega} \rightarrow 2$ existe um conjunto $H \in[\omega]^{\omega}$ que é $c$-monocromático?

Essa pergunta foi respondida negativamente por Erdös em 1952 [ER52]. A prova de Erdös da existência de uma bicoloração em $[\omega]^{\omega}$ sem um conjunto monocromático infinito é não construtiva, fazendo uso uma boa ordem para $[\omega]^{\omega}$. Ao invés de considerar colorações de subconjuntos de $\omega$, podemos considerar colorações para subconjuntos de algum conjunto infinito $A \subseteq \omega$. Agora, ainda que o Teorema Infinito de Ramsey não seja verdadeiro para $[\omega]^{\omega}$, é possível que seja verdadeiro para $[A]^{\omega}$, para alguns conjuntos infinitos $A \subseteq \omega$. Dizemos que os conjuntos infinitos $A \subseteq \omega$ para os quais toda coloração $c:[A]^{\omega} \rightarrow 2$ possui subconjunto infinito monocromático têm a Propriedade de Ramsey. Podemos enunciar essa definição em termos de partições:

Definição 4.6.2. Um conjunto $A \subseteq[\omega]^{\omega}$ tem a propriedade de Ramsey se existe $x \in[\omega]^{\omega}$ tal que $[x]^{\omega} \subseteq A$ ou $[x]^{\omega} \cap A=\emptyset$.

Embora nem todos os conjuntos tenham a Propriedade de Ramsey, a possibilidade de que conjuntos definiveis tenham essa propriedade foi especulada por Scott. Um resultado parcial nessa direção foi obtido por Galvin e Prikry em 1967 [GP73] (note que esse resultado só foi publicado em 1973), que introduziram a noção de conjunto completamente Ramsey, uma generalização da Propriedade de Ramsey, e provaram que todo boreliano é completamente Ramsey. A possibilidade de obter a consistência relativa para a Propriedade de Ramsey foi apontada por Friedman em 1967, em um seminário conduzido por Scott (Veja [Kan16]).

Definição 4.6.3. Seja $(s, x) \in[\omega]^{<\omega} \times[\omega]^{\omega}$ tal que $\max (s)<\min (x)$. Definimos

$$
[s, x]^{\omega}=\left\{z \in[\omega]^{\omega} \mid s \subseteq z \subseteq s \cup x\right\} .
$$

Convenção. Em todo o texto, ao escrever " $[s, x]^{\omega}$ " ficará sempre implícito que $s \in[\omega]^{<\omega}$, xin $[\omega]^{\omega}$ e $\max (s)<\min (x)$. Assim, ao escrever " $\forall[s, x]^{\omega}$ ", por exemplo, queremos dizer " $\forall s \in[\omega]<\omega \forall x \in$ $[\omega]^{\omega}(\max (s)<\min (x) \ldots)^{\prime \prime}$. 
Definição 4.6.4. Um conjunto $A \subseteq[\omega]^{\omega}$ é dito completamente Ramsey, ou Ramsey mensurável, se para todo $[s, x]^{\omega}$ existe $y \in[x]^{\omega}$ tal que $[s, y]^{\omega} \subseteq A$ ou $[s, y]^{\omega} \cap A=\emptyset$.

Em 1968 [Pri68] Prikry desenvolveu um forcing para cardinais mensuráveis: o forcing de Prikry. Mathias em 1969 [Mat69] desenvolveu uma versão desse forcing para $\omega$. O forcing de Mathias é formado por pares que devem aproximar esses conjuntos $[s, x]^{\omega}$. Não é surpreendente que as condições desse forcing sejam pares da forma $(s, x) \in[\omega]^{<\omega} \times[\omega]^{\omega}$, e a ordem deve ser tal que $(s, x) \leq(t, y) \leftrightarrow[s, x]^{\omega} \subseteq[t, y]^{\omega}$.

Definição 4.6.5. O forcing de Mathias é o forcing $(\mathbb{R}, \leq, \mathbb{1})$, onde

- $\mathbb{R}=\left\{(s, x) \in[\omega]^{<\omega} \times[\omega]^{\omega} \mid \max (s)<\min (x)\right\}$,

- $(s, x) \leq(t, y)$ se, e somente se, $t \subseteq s \wedge x \subseteq y \wedge s \backslash t \subseteq y, e$

- $\mathbb{1}=(\emptyset, \omega)$.

O forcing de Mathias é um forcing próprio que adiciona um número real, conhecido como real de Mathias.

Definição 4.6.6. Seja $G$ um filtro $\mathbb{R}$-genérico. Então o subconjunto de $\omega$ definido por $x_{G} \doteq \bigcup \pi G$ (i.e., a projeção na primeira coordenada) é dito real de Mathias.

Nosso objetivo agora é colocar o forcing de Mathias na forma idealizada, assim como fizemos nas seções anteriores. Precisamos de um $\sigma$-ideal candidato, que denotaremos por $\mathcal{R}^{0}$. Ao invés de considerar a topologia usual de $[\omega]^{\omega}$, usaremos a topologia de Ellentuck, que é mais natural para a nossa "nomenclatura". Silver em 1970 [Sil70] provou - usando técnicas de forcing e absolutividade que todo conjunto analítico é completamente Ramsey (usaremos uma adaptação dessa técnica de Silver na Seção 5.3.13). Ellentuck em 1974 [Ell74] obteve uma nova prova - sem usar forcing - de que os conjuntos analíticos são completamente Ramsey. Para isso, Ellentuck considerou os conjuntos da forma $[s, x]^{\omega}$ como abertos básicos para uma topologia em $[\omega]^{\omega}$.

Definição 4.6.7. O ideal $\mathcal{R}^{0}$ dos conjuntos completamente Ramsey-nulos é definido por

$$
\mathcal{R}^{0}=\left\{A \subseteq[\omega]^{\omega} \mid \forall[s, x]^{\omega} \exists y \in[\omega]^{\omega}\left([s, y]^{\omega} \cap A=\emptyset\right)\right\} .
$$

Teorema 4.6.8. $\mathcal{R}^{0}$ é um $\sigma$-ideal.

Demonstração. Sejam $\left\{A_{n} \mid n<\omega\right\}$ uma família em $\mathcal{R}^{0} \operatorname{com} A \doteq \bigcup_{n<\omega} A_{n}$ e $[s, x]^{\omega}$ arbitrário. Vamos construir um conjunto $y \in[x]^{\omega}$ recursivamente da seguinte forma:

Seja $x_{0} \in[x]^{\omega}$ tal que tal que $\left[s, x_{0}\right]^{\omega} \cap A_{0}=\emptyset$ e tome $a_{0}=\min \left(x_{0}\right)$. Seja $x_{1} \subseteq x_{0} \backslash\left\{a_{0}\right\}$ tal que $\left[s \cup\left\{a_{0}\right\}, x_{1}\right]^{\omega} \cap A_{1}=\emptyset$ e tome $a_{1}=\min \left(x_{1}\right)$. Construídos $x_{0} \supseteq x_{1} \supseteq \ldots \supseteq x_{n}$ e $a_{0}<a_{1}<$ $\ldots<a_{n}=\min \left(x_{n}\right)$, seja $x_{n+1} \in\left[x_{n} \backslash\left\{a_{0}, \ldots, a_{n}\right\}\right]^{\omega}$ tal que $\left[s \cup t, x_{n+1}\right]^{\omega} \cap A_{n+1}=\emptyset$ para todo $t \subseteq\left\{a_{0}, \ldots, a_{n}\right\}$ e tome $a_{n+1}=\min \left(x_{n+1}\right)$.

Considere $y=\left\{a_{n} \mid n<\omega\right\}$. Por construção, $[s, y]^{\omega} \cap A=\emptyset$, e então $A \in \mathcal{R}^{0}$.

Definição 4.6.9. A topologia de Ellentuck é a topologia em $[\omega]^{\omega}$ obtida tomando os conjuntos $[s, x]^{\omega}$ como abertos básicos.

Com a finalidade de construir um mergulho denso $\mathbb{R} \hookrightarrow \mathbb{P}_{\mathcal{R}^{0}}$ (note que aqui $\mathbb{P}_{\mathcal{R}^{0}}$ é na verdade $\left.\mathcal{B}\left([\omega]^{\omega}\right) \backslash \mathcal{R}^{0}\right)$, precisamos fazer uma indução boreliana. Começamos pela observação trivial de que a Ramsey mensurabilidade é fechada por complementação: 
Lema 4.6.10. Seja $A \subseteq \omega^{\omega}$. Então $A$ é completamente Ramsey se, e somente se, $[\omega]^{\omega} \backslash A$ é completamente Ramsey.

Teorema 4.6.11. Todo aberto (ou fechado) na topologia de Ellentuck é completamente Ramsey.

Demonstração. Seja $O$ aberto e considere as seguintes definições:

- $[s, x]^{\omega}$ é bom se $[s, y]^{\omega} \subseteq O$, para algum $y \in[x]^{\omega}$. Caso contrário, dizemos que $[s, x]^{\omega}$ é ruim.

- se $[s, x]^{\omega}$ é ruim, dizemos que $[s, x]^{\omega}$ é muito ruim se $[s \cup\{n\}, x / n]^{\omega}$ é ruim, para todo $n \in x$, onde $x / n=\{m \in x \mid m>n\}$.

Primeiro, note que se $[s, x]^{\omega}$ é ruim e $y \in[x]^{\omega}$, então $[s, y]^{\omega}$ também é ruim.

Afirmação: Se $[s, x]^{\omega}$ é ruim, então existe $y \in[x]^{\omega}$ tal que $[s, y]^{\omega}$ é muito ruim.

Demonstração. Suponha que a afirmação acima é falsa. Então existe $n_{0} \in x$ tal que $\left[s \cup\left\{n_{0}\right\}, x / n_{0}\right]^{\omega}$ é bom. Assim, existe $y_{0} \in\left[x / n_{0}\right]^{\omega}$ tal que $\left[s \cup\left\{n_{0}\right\}, y_{0}\right]^{\omega} \subseteq O$. Agora, definiremos recursivamente uma sequência crescente $\left(n_{k}\right)_{k<\omega}$ e uma sequência $\left(y_{k}\right)_{k<\omega}$ tais que para todo $k<\omega, n_{k} \in y_{k-1}$, $y_{k} \in\left[y_{k-1} / n_{k}\right]^{\omega}$ e $\left[s \cup\left\{n_{k}\right\}, y_{k}\right]^{\omega} \subseteq O$.

Suponha que definimos a sequência acima para todo $j<k$, para algum $k<\omega$. Note que $\left[s \cup\left\{n_{k-1}\right\}, y_{k-1} / n_{k-1}\right]^{\omega}=\left[s \cup\left\{n_{k-1}\right\}, y_{k-1}\right]^{\omega}$, pois $y_{k-1} \subseteq y_{k-2} / n_{k-1}$. Como $\left[s \cup\left\{n_{k-1}\right\}, y_{k-1}\right]^{\omega} \subseteq O$ por hipótese, então $\left[s, y_{k-1}\right]^{\omega}$ não é muito ruim, por definição. Assim, seja $n_{k} \in y_{k-1}\left(\log n_{k}>\right.$ $\left.n_{k-1}\right)$ tal que $\left[s \cup\left\{n_{k}\right\}, y_{k-1} / n_{k}\right]^{\omega}$ é bom. Agora, tome $y_{k} \in\left[y_{k-1} / n_{k}\right]^{\omega}$ tal que $\left[s \cup\left\{n_{k}\right\}, y_{k}\right]^{\omega} \subseteq O$.

Por fim, seja $y=\left\{n_{k} \mid k<\omega\right\}$ e note que $[s, y]^{\omega} \subseteq O$, contradizendo que $[s, x]^{\omega}$ é ruim. Isso encerra a prova da Afirmação.

Agora, se $[s, x]^{\omega}$ é bom, então terminamos. Se $[s, x]^{\omega}$ é ruim, vamos achar $y \in[x]^{\omega}$ tal que $[s, y]^{\omega} \cap O=\emptyset$ da seguinte forma:

Usando a afirmação anterior, sejam $y_{0} \in[x]^{\omega}$ tal que $\left[s, y_{0}\right]^{\omega}$ é muito ruim e $n_{0}=\min \left(y_{0}\right)$. Assim, $\left[s \cup\left\{n_{0}\right\}, y_{0} / n_{0}\right]^{\omega}$ é ruim. Novamente, aplique a afirmação anterior para obter $y_{1} \in\left[y_{0} / n_{0}\right]^{\omega}$ tal que $\left[s \cup\left\{n_{0}\right\}, y_{1}\right]^{\omega}$ é muito ruim. Então $\left[s \cup\left\{n_{0}, n_{1}\right\}, y_{1} / n_{1}\right]$ é ruim, onde $n_{1}=\min \left(y_{1}\right)$. Repetindo esse processo via recursão obtemos uma sequência decrescente $x \supseteq y_{0} \supseteq \cdots \operatorname{com} n_{k}=\min \left(y_{k}\right)$ tal que para todo $t \subseteq\left\{n_{0}, \ldots, n_{k-1}\right\},\left[s \cup t, y_{k}\right]^{\omega}$ é muito ruim e, portanto, $\left[s \cup t, y_{k} / n_{k}\right]^{\omega}$ é ruim, para todo $t \subseteq\left\{n_{0}, \ldots, n_{k}\right\}$. Seja então $y=\left\{n_{k} \mid k<\omega\right\}$. Vamos ver que $[s, y]^{\omega} \cap O=\emptyset$.

De fato, suponha $[s, y]^{\omega} \cap O \neq \emptyset$. Como $O$ e $[s, y]^{\omega}$ são abertos, seja $\left[s^{\prime}, y^{\prime}\right]^{\omega} \subseteq[s, y]^{\omega} \cap O$. Então existe algum $k<\omega$ tal que $s^{\prime}=s \cup t$ com $t \subseteq\left\{n_{0}, \ldots, n_{k}\right\}$ e $y^{\prime} / n_{k} \subseteq y_{k} / n_{k}$. Assim, como $\left[s \cup t, y^{\prime} / n_{k}\right]^{\omega} \subseteq O$, temos que $\left[s \cup t, y_{k} / n_{k}\right]^{\omega}$ é bom, o que é uma contradição.

Corolário 4.6.12. Todo boreliano na topologia de Ellentuck é completamente Ramsey.

Corolário 4.6.13. $\mathbb{P}_{\mathcal{R}^{0}}$ e $\mathbb{R}$ são forcing-equivalentes.

Demonstração. O mapa $(s, x) \mapsto[s, x]^{\omega}$ é um mergulho denso de $\mathbb{R}$ em $\mathbb{P}_{\mathcal{R}^{0}}$.

Ellentuck mostrou que os conjuntos completamente Ramsey são exatamente os conjuntos Baire mensuráveis na topologia de Ellentuck. Embora isso não seja necessário para o nosso objetivo, como esse resultado é mencionado na literatura e a prova não é muito difícil, ele é apresentado a seguir.

Lema 4.6.14 ([Ell74]). Seja $A \subseteq[\omega]^{\omega}$. São equivalentes:

1. A é raro.

2. A é completamente Ramsey-nulo. 
3. A é magro.

Demonstração. Provaremos 1. $\Leftrightarrow 2$., e como obviamente $1 . \Rightarrow 3$, finalizaremos provando que $3 . \Rightarrow$ 1.

- (1. $\Leftrightarrow 2$.) $A \subseteq[\omega]^{\omega}$ é raro se, e somente se, para qualquer aberto da forma $[s, x]^{\omega}$ existe $[t, y]^{\omega}$ tal que $[t, y]^{\omega} \cap A=\emptyset$. Assim, se A é completamente Ramsey-nulo então é trivialmente raro. Reciprocamente, suponha $A$ raro. Temos que $\bar{A}$ é completamente Ramsey, pelo Teorema 4.6.11. Assim, para todo $[s, x]^{\omega}$ existe $y \in[x]^{\omega}$ tal que $[s, y]^{\omega} \subseteq \bar{A}$ ou $[s, y]^{\omega} \cap \bar{A}=\emptyset$. O primeiro caso não pode ocorrer pois $\operatorname{int}(\bar{A})=\emptyset$. Logo, $\emptyset=[s, y]^{\omega} \cap \bar{A} \supseteq[s, y]^{\omega} \cap A$ e, portanto, $A$ é completamente Ramsey-nulo.

- (3. $\Rightarrow$ 1.) Suponha $A \subseteq[\omega]^{\omega}$ magro e seja $\left\{A_{n} \mid n<\omega\right\}$ uma família de raros tal que $A=\bigcup_{n<\omega} A_{n}$. Pelo ítem anterior, cada $A_{n}$ é completamente Ramsey-nulo e, como $\mathcal{R}^{0}$ é um $\sigma$-ideal (Teorema 4.6.8), $A$ é completamente Ramsey-nulo, portanto um conjunto raro.

Teorema 4.6.15 ([Ell74]). $A \subseteq[\omega]^{\omega}$ é completamente Ramsey se, e somente se, $A$ é Baire mensurável na topologia de Ellentuck.

Demonstração. Suponha $A$ completamente Ramsey e seja $B=A \backslash \operatorname{int}(A)$. Vamos verificar que $B$ é raro.

De fato, se $B$ não é raro, então existe $[s, x]^{\omega} \subseteq \bar{B}$. Primeiro, note que $[s, y]^{\omega} \subseteq \bar{B}$ e, por um argumento simples de topologia, $[s, y]^{\omega} \cap B \neq \emptyset$. Agora, seja $y \in[x]^{\omega}$ tal que $[s, y]^{\omega} \subseteq A$ ou $[s, y]^{\omega} \cap A=\emptyset$. Se $[s, y]^{\omega} \cap A=\emptyset$, então $[s, y]^{\omega} \cap B=\emptyset$, absurdo. Se $[s, y]^{\omega} \subseteq A$, então $[s, y]^{\omega} \subseteq \operatorname{int}(A)$, e então $[s, y]^{\omega} \cap B=\emptyset$, absurdo. Assim, $B$ é raro e $A=B \cup \operatorname{int}(A)=B \Delta \operatorname{int}(A)$. Logo, $A$ é Baire mensurável.

Reciprocamente, suponha que $A$ é Baire mensurável e sejam $B$ um raro (portanto magro e completamente Ramsey-nulo, pelo Teorema 4.6.14) e $O$ um aberto tais que $A=B \Delta O$. Seja $[s, x]^{\omega}$ qualquer e considere $y \in[x]^{\omega}$ tal que $[s, y]^{\omega} \cap B=\emptyset$. Como $O$ é aberto, então é completamente Ramsey. Seja então $z \in[y]^{\omega}$ tal que $[s, z]^{\omega} \subseteq O$ ou $[s, z]^{\omega} \cap O=\emptyset$. No primeiro caso, $[s, z]^{\omega} \subseteq A$, e no segundo, $[s, z]^{\omega} \cap A=\emptyset$.

\subsection{Fazendo doughnuts com árvores de Silver}

Em 2000 [DPH00], investigando propriedades de partição da reta, DiPrisco e Henle introduziram a noção de doughnut e a propriedade doughnut. Essa noção de regularidade é bastante similar à Propriedade de Ramsey (de fato, é um enfraquecimento da propriedade de Ramsey).

Definição 4.7.1. Sejam $a \subseteq b \subseteq \omega$ tais que $b \backslash a \in[\omega]^{\omega}$. O conjunto

$$
D \doteq\left\{x \in[\omega]^{\omega} \mid a \subseteq x \subseteq b\right\}
$$

é dito doughnut (nesse caso, um (a,b)-doughnut).

Note que um $(a, b)$-doughnut nunca é vazio, pois $b$ sempre é um elemento. Agora, sejam $a \subseteq b \subseteq \omega$ e suponha que existe $x \in[\omega]^{\omega}$ tal que $|x \backslash a|=\aleph_{0}$ ou $|b \backslash x|=\aleph_{0}$. Assim, $|b \backslash a|=|(b \backslash x) \cup(x \backslash a)|=$ $|b \backslash x|+|x \backslash a|=\aleph_{0}$, e então podemos definir um $(a, b)$-doughnut.

Definição 4.7.2. Sejam $a \subseteq b \subseteq \omega$. Definimos

$$
[a, b]^{\omega}=\left\{x \in[\omega]^{\omega} \mid a \subseteq x \subseteq b \wedge\left(|x \backslash a|=\aleph_{0} \vee|b \backslash x|=\aleph_{0}\right)\right\}
$$


Assim, $[a, b]^{\omega}$ como acima, ou é o conjunto vazio ou é um $(a, b)$-doughnut. Estamos usando quase a mesma notação que usamos para os abertos da topologia de Ellentuck, mas note que reservamos a primeira metade do alfabeto para os doughnuts $[a, b]^{\omega}$, enquanto reservamos a última metade do alfabeto para os abertos da topologia de Ellentuck $[s, x]^{\omega}$. Agora, podemos então definir a propriedade doughnut:

Definição 4.7.3. Seja $A \subseteq[\omega]^{\omega}$.

1. Dizemos que A tem a propriedade doughnut quando existe um doughnut $[a, b]^{\omega}$ tal que $[a, b]^{\omega} \subseteq A$ ou $[a, b]^{\omega} \cap A=\emptyset$.

2. Dizemos que A é completamente doughnut se para todo doughnut $[a, b]^{\omega}$ existe um doughnut $[c, d]^{\omega} \subseteq[a, b]^{\omega}$ tal que $[c, d]^{\omega} \subseteq A$ ou $[c, d]^{\omega} \cap A=\emptyset$.

Da mesma forma que no caso da Ramsey mensurabilidade, temos naturalmente um ideal associado à propriedade "completamente doughnut".

Definição 4.7.4. O ideal $\mathcal{D}^{0}$ dos conjuntos completamente doughnut-nulos é definido por

$$
\mathcal{D}^{0}=\left\{A \subseteq[\omega]^{\omega} \mid \forall[a, b]^{\omega} \neq \emptyset \exists[c, d]^{\omega} \neq \emptyset\left([c, d]^{\omega} \subseteq[a, b]^{\omega} \wedge[c, d]^{\omega} \cap A=\emptyset\right)\right\}
$$

Ainda não estamos preparados para provar que $\mathcal{D}^{0}$ é uma $\sigma$-ideal. Logo faremos isso com o auxílio das árvores de Silver.

Assim como no caso de caso da Ramsey mensurabilidade, poderíamos considerar alguma noção de forcing cujas condições da forma $(a, b)$, com $b \backslash a \in[\omega]^{\omega}$, que aproximam os conjuntos da forma $[a, b]^{\omega}$, para alguma ordem adequada. Ao invés disso, usaremos o forcing de Silver, cujas condições são árvores uniformes perfeitas (também chamadas de árvores de Silver). Essa relação entre a propriedade doughnut e o forcing de Silver foi explorada em 2003 [Hal03] por Halbeisen. Em analogia com o trabalho de Ellentuck, Halbeisen desenvolveu a topologia doughnut. Com essa topologia doughnut em $[\omega]^{\omega}$, provaremos que o forcing $\mathbb{P}_{\mathcal{D}^{0}}$ é a forma idealizada do forcing de Silver.

Definição 4.7.5. A topologia doughnut é a topologia em $[\omega]^{\omega}$ obtida tomando como base $\emptyset,[\omega]^{\omega}$, e os conjuntos da forma

$$
(a, b)^{\omega} \doteq\left\{x \in[\omega]^{\omega} \mid a \subseteq x \subseteq b \wedge\left(|x \backslash a|=|b \backslash x|=\aleph_{0}\right)\right\},
$$

onde $a \subseteq b \subseteq \omega$.

Nosso objetivo é provar que os borelianos da topologia doughnut são completamente doughnut. Faremos isso por indução boreliana, começando pelos abertos.

Lema 4.7.6. Para todo doughnut $[a, b]^{\omega}$, existem $c, d \in[a, b]^{\omega}$ tais que

$$
\emptyset \neq(c, d)^{\omega} \subseteq[c, d]^{\omega} \subseteq(a, b)^{\omega} \subseteq[a, b]^{\omega} .
$$

Demonstração. Considere $c, d \in[a, b]^{\omega}$ tais que $a \subseteq c \subseteq d \subseteq b$ e $c \backslash a, d \backslash c$ e $b \backslash c$ são infinitos.

Teorema 4.7.7. Todo aberto (ou fechado) é completamente doughnut.

Demonstração. Se $O \subseteq[\omega]^{\omega}$ é aberto, então $O$ é a união de abertos básicos. Assim, para qualquer doughnut $[a, b]^{\omega}$, temos que $O \cap(a, b)^{\omega}$ é aberto. Se $O \cap(a, b)^{\omega}=\emptyset$, existe $[c, d]^{\omega} \subseteq(a, b)^{\omega}$ e, portanto, $O \cap[c, d]^{\omega}=\emptyset$. Se $O \cap(a, b)^{\omega} \neq \emptyset$, existe um doughnut $[c, d]^{\omega} \subseteq O \cap(a, b)^{\omega}$. Segue que $O$ é completamente doughnut. 
Para finalizar a indução boreliana, resta provar que $\mathcal{D}^{0}$ é um $\sigma$-ideal. Para isso, precisamos do forcing de Silver.

Definição 4.7.8. Seja $T \subseteq 2^{<\omega}$ uma árvore.

1. T é uma árvore uniforme se

$$
\forall s, t \in T\left(|s|=|t| \rightarrow\left(s^{\wedge} i \in T \leftrightarrow t^{\frown} i \in T\right)\right)
$$

para todo $i \in\{0,1\}$.

2. T é uma árvore de Silver se é uma árvore perfeita e uniforme.

Definição 4.7.9. O forcing de Silver é o forcing $(\mathbb{V}, \leq, \mathbb{1})$, onde

- as condições em $\mathbb{V}$ são árvores uniformes perfeitas,

- $a$ ordem $\leq e ́$ a inclusão direta $\subseteq$, e

- o maior elemento é $\mathbb{1}=2^{<\omega}$.

Assim como os forcings anteriores, o forcing de Silver adiciona um real genérico - real de Silver - e é próprio.

Teorema 4.7.10 ([Hal03]). Existe um isomorfismo do forcing de Silver no conjunto de todos os doughnuts, com a ordem da inclusão. O único doughnut associado a uma árvore de Silver $T$ será denotado por $\operatorname{dough}(T)$.

Demonstração. Defina, para $y \in 2^{\omega}, \chi^{-1}(y)=\{n \in \omega \mid y(n)=1\}$. Agora, sejam $T$ uma árvore de Silver e

$$
\operatorname{dough}(T) \doteq\left\{\chi^{-1}(y) \mid y \in[T]\right\} \cap[\omega]^{\omega} .
$$

Vamos mostrar que $\operatorname{dough}(T)=[a, b]^{\omega}$, onde $a=\bigcap_{y \in[T]} \chi^{-1}(y)$ e $b=a \cup \operatorname{Splev}(T)$. Primeiro, tome $y \in[T]$ tal que $\chi^{-1}(y) \in[\omega]^{\omega}$. Claramente $a \subseteq \chi^{-1}(y)$ e $b \backslash a \in[\omega]^{\omega}$, pois $T$ é uma árvore perfeita. Além disso, se $n \in \chi^{-1}(y)$ e $n \in a$, então trivialmente $\chi^{-1}(y) \subseteq b$. Se $n \notin a$, então $y(n)=1$ e existe $z \in[T]$ tal que $z(n)=0$. Ora, $y_{n} \doteq y\left\lceil n \in T\right.$ e $z_{n} \doteq z\left\lceil n \in T\right.$. Assim, $y_{n} 1 \in T$ e $z_{n} 0 \in T$. Como $T$ é uniforme e $\left|y_{n}\right|=\left|z_{n}\right|$, isso implica que $y_{n} 0 \in T$ e $z_{n}^{\frown} 1 \in T$. Logo, $n \in \operatorname{Splev}(T)$ e, portanto, $\chi^{-1}(y) \subseteq b$. Segue que $\chi^{-1}(y) \in[a, b]^{\omega}$.

Agora, tome $y \in[a, b]^{\omega}$ e note que $y \in[T]$. Considere $z \doteq \chi_{y}$ a função característica de $y$. Temos que

$$
n \in y \leftrightarrow z(n)=1 \leftrightarrow n \in \chi^{-1}(z) .
$$

Logo, $y=\chi^{-1}(z) \in[\omega]^{\omega}$, e então $y \in \operatorname{dough}(T)$.

Para provar que a correspondência $T \mapsto \operatorname{dough}(T)$ é sobrejetora, sejam $a \subseteq b \subseteq \omega$ tais que $b \backslash a \in[\omega]^{\omega}$ e defina $T \subseteq 2^{<\omega}$ como

$$
T=\left\{y\left\lceil n \mid y \in 2^{\omega} \wedge a \subseteq \chi^{-1}(y) \subseteq b\right\}\right.
$$

e note que

$$
[T]=\left\{y \in 2^{\omega} \mid a \subseteq \chi^{-1}(y) \subseteq b\right\}=\left\{y \in 2^{\omega} \mid y\lceil a=1 \wedge y\lceil\omega \backslash b=0\} .\right.
$$

Vamos mostrar que $T$ é uma árvore de Silver. Sejam $y, z \in 2^{\omega}, n<\omega$ tais que $s \doteq y\lceil n \in T$ e $t \doteq z\lceil n \in T(n=|s|=|t|)$. 
- Se $n \in a$, então $y(n)=z(n)=1 \mathrm{e}$, portanto, $s^{\frown} 1, t^{\frown} 1 \in T$, e $s^{\frown} 0, t^{\frown} 0 \notin T$.

- Se $n \in \omega \backslash b$, então $y(n)=z(n)=0$ e, portanto, $s^{\frown} 0, t^{\frown} 0 \in T$, e $s^{\frown} 1, t^{\frown} 1 \notin T$.

- Se $n \in b \backslash a$, então $s^{\frown} i, t^{\frown} i \in T$, para $i<2$.

Falta mostrar que $T$ é uma árvore perfeita. Sejam então $y \in 2^{\omega}$ e $n<\omega$ tais que $s \doteq y\lceil n \in T$. Como $b \backslash a$ é infinito, seja $m>n$ o menor natural em $b \backslash a$. Temos que $t \doteq y\lceil m$ é tal que $t^{\frown} 0, t^{\frown} 1 \in T$.

Por fim, se $T, S$ são árvores de Silver, é fácil ver que

$$
T \leq S \leftrightarrow \operatorname{dough}(T) \subseteq \operatorname{dough}(S) .
$$

Teorema 4.7.11. $\mathcal{D}^{0}$ é um $\sigma$-ideal.

Corolário 4.7.12. Todo boreliano da topologia doughnut é completamente doughnut.

Corolário 4.7.13. $\mathbb{P}_{\mathcal{D}^{0}} e \mathbb{V}$ são forcing-equivalentes.

Demonstração. O mapa $T \mapsto \operatorname{dough}(T)$ é um mergulho denso de $\mathbb{V}$ em $\mathbb{P}_{\mathcal{D}^{0}}$.

Como os conjuntos completamente Ramsey são os conjuntos Baire mensuráveis na topologia de Ellentuck, não é de se surpreender que os conjuntos completamente doughnuts sejam os conjuntos Baire mensuráveis na topologia doughnut. Novamente, apesar de interessante, esse resultado não é relevante para o nosso objetivo. As provas desses resultados podem ser encontradas em [Hal03].

Lema 4.7.14 ([Hal03]). Seja $A \subseteq[\omega]^{\omega}$. São equivalentes:

1. A é raro.

2. A é completamente doughnut-nulo.

3. A é magro.

Teorema 4.7.15. Seja $A \subseteq[\omega]^{\omega}$. A é completamente doughnut se, e somente se, $A$ é Baire mensurável na topologia doughnut. 


\section{Capítulo 5}

\section{Regularidade idealizada}

\subsection{Propriedades gerais do forcing idealizado}

O forcing idealizado captura em uma única definição muitas noções clássicas de forcing, como as consideradas no capítulo anterior. Os forcings que consideramos adicionam um número real, com certas propriedades que dependem da combinatória de cada noção de forcing. Analisaremos a adição de um real genérico pelo forcing idealizado, mas antes precisamos fazer algumas considerações sobre nomes para borelianos codificados no modelo inicial.

Sejam $J$ um $\sigma$-ideal, $G$ um filtro $\mathbb{P}_{J}$-genérico e $B$ um boreliano codificado em $\mathrm{V}$, com código c. Convencionamos que uma fórmula do tipo $x \in B^{\mathrm{V}[G]}$ é na verdade $x \in B_{c}^{\mathrm{V}[G]}$. Entendemos esse processo da seguinte forma: consideramos o código $c$ de $B$, e então construímos $B_{c}$ em $V[G]$, que é como $\mathrm{V}[G]$ enxerga o boreliano $B$, então verificamos se $x \in B_{c}^{\mathrm{V}[G]}$. Nesse caso, deve existir uma condição $C$ que força essa afirmação. Por ora, vamos denotar por $B: \mathrm{BC} \rightarrow \mathcal{B}\left(\omega^{\omega}\right)$ a decodificação de Borel, para facilitar a discussão (i.e., $B(c)=B_{c}$ ). Se $x \in B(c)^{\mathrm{V}[G]}$, então existe $C \in \mathbb{P}_{J}$ tal que $C \Vdash \dot{x} \in \check{B}(\check{c})$. Denotaremos esse nome $\check{B}(\check{c})$ por $B^{*}$.

Definição 5.1.1. Sejam $J$ um $\sigma$-ideal, $G$ um filtro $\mathbb{P}_{J}$-genérico e $B$ um boreliano codificado em $\mathrm{V}$, com código $c$. Denotamos o $\mathbb{P}_{J}$-nome para $B_{c}$ por $B^{*}$.

O seguinte teorema foi enunciado e provado no contexto do forcing aleatório por Solovay em [Sol70], e no contexto do forcing idealizado por Zapletal em [Zap04].

Teorema 5.1.2 ([Sol70, Zap04]). Seja J um $\sigma$-ideal. Se $G$ é um filtro $\mathbb{P}_{J}$-genérico, então existe um único real $x_{G} \in \mathrm{V}[G]$ tal que para todo conjunto boreliano $B$ codificado em $V, x_{G} \in B^{\mathrm{V}[G]}$ se, e somente se, $B^{V} \in G$. Além disso, $G$ é definivel em $V\left[x_{G}\right]$ e, portanto, $V\left[x_{G}\right]=\mathrm{V}[G]$.

Demonstração. Primeiro vamos mostrar que os abertos básicos da forma $\mathcal{O}_{s}$ têm a propriedade que queremos. Isto é, existe $x_{G}$ tal que

$$
x_{G} \in \mathcal{O}_{s}^{\mathrm{V}[G]} \leftrightarrow \mathcal{O}_{s} \in G .
$$

Faremos uma construção recursiva, argumentando em $\mathrm{V}[G]$, da seguinte forma:

- $s_{0}=\emptyset$. Assim, $\mathcal{O}_{s_{0}}=\omega^{\omega} \in G$.

- Suponha que para $n \in \omega$ construimos uma sequência $\left(s_{i}\right)_{i \leq n}$ tal que $s_{i} \in \omega^{i}, \mathcal{O}_{s_{i}} \in G$ e $s_{j} \subseteq s_{i}$, para todos $j \leq i \leq n$. Note que o conjunto

$$
D_{n} \doteq\left\{B \in \mathbb{P}_{J} \mid \exists k\left(B \subseteq \mathcal{O}_{\widehat{s} \widehat{n} k}\right)\right\}
$$

é denso abaixo de $\mathcal{O}_{n}$.

De fato, se $B \subseteq \mathcal{O}_{s_{n}}$, então $B=B \cap\left(\bigcup_{k \in \omega} \mathcal{O}_{\widehat{s_{n}} \widehat{ }}\right)=\bigcup_{k \in \omega}\left(B \cap \mathcal{O}_{s_{\tilde{n}} k}\right)$. Logo, para algum $k \in \omega$, devemos ter $B \cap \mathcal{O}_{\widehat{s} \widehat{n}} \in \mathbb{P}_{J}$. 
Portanto, devem existir $k \in \omega$ e $B$ um borealiano $I$-positivo tais que $B \cap \mathcal{O}_{s_{\widehat{n}} k} \in G$ e, portanto, $\mathcal{O}_{s_{n} k} \in G$

Assim, defina $s_{n+1}=\widehat{s_{n}} k$ para $k$ como acima. Temos então definida uma sequência $\left(s_{n}\right)_{n \in \omega}$ de elementos de $\omega^{<\omega}$ de modo que para todo $n \in \omega, s_{n} \subseteq s_{n+1}, \mathcal{O}_{s_{n+1}} \subseteq \mathcal{O}_{s_{n}}$ e $\mathcal{O}_{s_{n}} \in G$.

- Defina $x_{G}=\bigcup\left\{s_{n}: n \in \omega\right\}$.

Afirmamos que esse $x_{G} \in \mathrm{V}[G]$ é como queremos.

De fato, sejam $s \in \omega^{<\omega}$ e $\mathcal{O}_{s}$ aberto básico. Se $x_{G} \in \mathcal{O}_{s}^{\mathrm{V}[G]}$, então existe $n \in \omega$ tal que $s \subseteq s_{n}$. Assim, $\mathcal{O}_{s_{n}} \subseteq \mathcal{O}_{s}$ e, como $\mathcal{O}_{s_{n}} \in G$, então $\mathcal{O}_{s} \in G$. Reciprocamente, se $x_{G} \notin \mathcal{O}_{s}^{\mathrm{V}[G]}$, então existem $n \in \omega$ e $i \in \operatorname{dom}\left(s_{n}\right) \cap \operatorname{dom}(s)$ tais que $s_{n}(i) \neq s$ e, portanto, $s, s_{n}$ são funções incompatíveis, o que implica $\mathcal{O}_{s_{n}} \cap \mathcal{O}_{s}=\emptyset \notin G$. Como $\mathcal{O}_{s_{n}} \in G$, não podemos ter $\mathcal{O}_{s} \in G$.

Agora, considere o conjunto $\mathcal{B}^{\prime}=\left\{B \in \mathbb{P}_{J} \mid B \Vdash \dot{x}_{G} \in B^{*}\right\}$. Pelo argumento acima, temos que $\mathcal{B}^{\prime}$ contém os abertos básicos. Seja $\left(B_{n}\right)_{n \in \omega}$ uma família em $\mathcal{B}^{\prime}$. Se $B=\bigcap_{n \in \omega} B_{n}$, como $B \subseteq B_{n}$ para todo $n \in \omega$, então $B \Vdash \dot{x}_{G} \in B_{n}^{*}$, para todo $n \in \omega$ e, portanto, $B \Vdash \dot{x}_{G} \in \bigcap_{n \in \omega} B_{n}^{*}=B^{*}$. Se $B=\bigcup_{n \in \omega} B_{n}$, note que $\left\{C \leq B \mid C \Vdash \dot{x}_{G} \in B^{*}\right\}$ é denso abaixo de $B$. De fato, se $C \leq B$, então $C=C \cap B=\bigcup_{n \in \omega} C \cap B_{n} \notin J$ e, portanto, deve existir algum $n \in \omega$ tal que $C \cap B_{n}$ é um borealino $J$-positivo. Como $B_{n} \Vdash \dot{x}_{G} \in \dot{B}_{n}^{*}$, então $C \cap B_{n} \Vdash \dot{x}_{G} \in \dot{B}_{n}^{*} \subseteq B^{*}$. Logo, $B \Vdash \dot{x}_{G} \in B^{*}$. Assim, $\mathcal{B}^{\prime}=\mathbb{P}_{J}$, e isso prova que se $B \in G$ então $x_{G} \in B^{\mathrm{V}[G]}$.

Para a recíproca, suponha que $x_{G} \in B^{\mathrm{V}}[G]$ e seja $C \in G$ tal que $C \Vdash \dot{x}_{G} \in B^{*}$. Note que $C \backslash B \in J$. De fato, se $C \backslash B$ fosse $J$-positivo, teríamos $C \backslash B \Vdash \dot{x}_{G} \in C^{*} \backslash B^{*}$ e, portanto, $C \backslash B$ seria uma extensão de $C$ forçando $\dot{x}_{G} \notin B^{*}$, absurdo. Agora, note que se não tivéssemos $B \in G$, então teríamos $\omega^{\omega} \backslash B \in G$ e, portanto, $C \backslash B=C \cap\left(\omega^{\omega} \backslash B\right) \in G$, o que pelo comentário anterior é um absurdo. Logo, $B \in G$.

Agora, para ver que $G$ é definível em $V\left[x_{G}\right]$, basta verificar que

$$
G=\left\{B \in \mathbb{P}_{J} \mid \operatorname{code}(B) \in V \wedge x_{G} \in B^{V\left[x_{G}\right]}\right\} .
$$

Da mesma forma que podemos considerar filtros que são genéricos sobre algum modelo interno $\in$-transitivo, podemos também considerar reais que são $\mathbb{P}_{J}$-genéricos para esses modelos internos.

Definição 5.1.3. Sejam $J$ um $\sigma$-ideal $M$ um modelo $\in$-transitivo para $Z F C$. Dizemos um real $x$ é $\mathbb{P}_{J}$-genérico sobre $M$ se existe algum $G$ filtro $\mathbb{P}_{J}^{M}$-genérico sobre $M$ tal que para qualquer boreliano $B$ codificado em $M$, temos

$$
x \in B^{M[G]} \leftrightarrow B^{M} \in G .
$$

É possível mostrar, de forma análoga à prova do Teorema 5.1.2, que se $G$ é $\mathbb{P}_{J}^{M}$-genérico sobre $M$ então existe um único $x$ que é $\mathbb{P}_{J}$-genérico sobre $M$.

Lema 5.1.4. Sejam $J$ um $\sigma$-ideal e $M$ um modelo $\in$-transitivo para $Z F C$. Então $x$ é um real $\mathbb{P}_{J}$-genérico sobre $M$ se, e somente se, $x \in \bigcup D$, para todo $D \in M$ denso em $\mathbb{P}_{J}^{M}$.

Demonstração. Sejam $x$ um $\mathbb{P}_{J}$-genérico sobre $M, G \subseteq \mathbb{P}_{J}^{M}$ o correspondente filtro $\mathbb{P}_{J}^{M}$-genérico sobre $M$ e $D \in M$ um denso qualquer em $\mathbb{P}_{J}^{M}$. O filtro $G$ pode ser definido da seguinte forma:

$$
G=\left\{B \in \mathbb{P}_{J}^{M} \mid c(B) \in M \wedge x \in B\right\} .
$$

Agora, como $G$ é $\mathbb{P}_{J}^{M}$-genérico sobre $M$, então $G \cap D \neq \emptyset$ e, portanto, existe $B \in D$ tal que $x \in B$. Logo, $x \in \bigcup D$. 
Definição 5.1.5. Sejam $J$ um $\sigma$-ideal e $M$ um modelo, não necessariamente transitivo, de ZF-P. Dizemos que $x$ é um $\left(M, \mathbb{P}_{J}\right)$-genérico se $G_{x} \doteq\left\{B \in \mathbb{P}_{J} \cap M \mid x \in B\right\}$ é um filtro $\left(M, \mathbb{P}_{J}\right)$-genérico. Ou, equivalente, $x \in \bigcup(D \cap M)$ para todo $D \in M$ denso em $\mathbb{P}_{J} \cap M$.

Todas as noções de forcing consideradas no capítulo anterior são próprias, e a forma idealizada preserva essa propriedade. Zapletal [Zap04] provou uma boa caracterização para forcings idealizados próprios: as condições $\left(M, \mathbb{P}_{J}\right)$-masters são conjuntos borelianos $J$-positivos.

Teorema 5.1.6. Seja J um $\sigma$-ideal. São equivalentes:

1. $\mathbb{P}_{J}$ é forcing próprio.

2. Para todo submodelo elementar enumerável $M \preceq H_{\lambda}$ de uma subestrutura suficientemente grande e para todo $B \in \mathbb{P}_{J} \cap M$, o conjunto

$$
C \doteq\{x \in B \mid x \text { é M-genérico }\}
$$

é um boreliano J-positivo.

Demonstração. Suponha que $\mathbb{P}_{J}$ é próprio e sejam $M \preceq H_{\lambda}$ um submodelo elementar enumerável de uma estrutura suficientemente grande e $B \in \mathbb{P}_{J} \cap M$.

Note que

$$
C=B \cap \bigcap\left\{\bigcup(D \cap M): D \in M \text { é denso em } \mathbb{P}_{J}\right\}
$$

é boreliano, pois $M$ é enumerável.

Agora, como $\mathbb{P}_{J}$ é próprio, seja $D \subseteq B$ condição $M$-master. Isto é,

$D \Vdash \dot{x}_{G}$ é um real $M$-genérico.

Assim, pela definição de $C$, temos que $D \Vdash \dot{x}_{G} \in \dot{C}$. Logo, pelo Teorema 5.1.2, $C$ deve ser $I$-positivo.

Reciprocamente, suponha que $C$ é $I$-positivo. Então, novamente pelo Teorema 5.1.2, temos $C \Vdash \dot{x}_{G} \in \dot{C}$ e, portanto, $C \Vdash \dot{x}_{G}$ é real $M$-genérico. Logo, $C$ é a condição $M$-master abaixo de $B$ e $\mathbb{P}_{J}$ é próprio.

A primeira implicação importante dessa caracterização para forcings idealizados próprios é que cada real da extensão genérica é a imagem do real genérico por alguma função boreliana codificada no modelo inicial. Essa propriedade é chamada de leitura boreliana para nomes, e foi provada por Zapletal em 2008 [Zap08].

Teorema 5.1.7 (Leitura boreliana de nomes [Zap08]). Sejam $J$ um $\sigma$-ideal tal que $\mathbb{P}_{J}$ é forcing próprio e $B$ uma condição tal que $B \Vdash \dot{x}$ é um número real. Então existe uma condição $C \leq B$ e uma função boreliana $f: C \rightarrow \omega^{\omega}$ tais que $C \Vdash \dot{x}=\check{f}\left(\dot{x}_{G}\right)$.

Há outra propriedade comum entre os ideais dos forcings idealizados que consideramos no capítulo anterior. A saber, todos eles são Borel gerados. Um $\sigma$-ideal é Borel gerado se todo elemento está contido em algum boreliano desse $\sigma$-ideal.

Definição 5.1.8. Seja $J$ um $\sigma$-ideal. Dizemos que $J$ é Borel gerado se para todo $A \in J$ existe um boreliano $B \in J$ tal que $A \subseteq B$.

\section{Exemplo 5.1.9.}

1. O ideal ctbl é Borel gerado, pois todo conjunto de reais enumerável é boreliano (é a união enumerável de unitários). 
2. O ideal null é Borel gerado por definição.

3. O ideal meag é Borel gerado. De fato, meag é o $\sigma$-ideal gerado pelos conjuntos raros. Se $F$ é raro, então $F \subseteq \mathrm{cl}(F)$, sendo o último um boreliano (fechado) raro.

4. $O$ ideal $K_{\sigma}$ é Borel gerado, sendo o $\sigma$-ideal gerado pelos conjuntos $\sigma$-compactos, que são borelianos.

5. O ideal $\mathcal{L}^{0}$ é Borel gerado, por definição.

6. Os ideais $\mathcal{R}^{0}$ e $\mathcal{D}^{0}$ são Borel gerados nas topologias de Ellentuck e doughnut, respectivamente.

Restringir nossos argumentos apenas aos $\sigma$-ideais Borel gerados é crucial para definir um $\sigma$ ideal auxiliar que será importante nos nossos argumentos: o $\sigma$-ideal $\mathcal{N}_{J}$. Porém, é sempre possível considerar a borelização de um $\sigma$-ideal, que é o $\sigma$-ideal formado pelos conjuntos que estão contidos em algum boreliano desse $\sigma$-ideal.

Está decidido que nossa definição de regularidade idealizada (i.e., regularidade para forcings da forma $\mathbb{P}_{J}$ ) será feita para $\sigma$-ideais Borel gerados e forcings próprios. Com base no capítulo anterior, é natural definir a regularidade do tipo dicotomia da seguinte forma:

Definição 5.1.10. Seja $J$ um $\sigma$-ideal Borel gerado tal que $\mathbb{P}_{J}$ é forcing próprio. Dizemos que $A \subseteq \omega^{\omega}$ satisfaz a $\mathbb{P}_{J}$-dicotomia se $A \in J$ ou se existe um boreliano $J$-positivo $B$ tal que $B \subseteq A$.

Por outro lado, temos duas opções naturais para a regularidade do tipo mensurabilidade:

1. Similarmente ao caso da Lebesgue mensurabilidade e da Baire mensurabilidade, podemos dizer que $A$ é $\mathbb{P}_{J}$-mensurável se existe um boreliano $B$ tal que $A \Delta B \in J$.

2. Similarmente ao caso das outras noções de regularidade, podemos dizer que $A$ é $\mathbb{P}_{J}$-mensurável se para todo boreliano $J$-positivo $B$, existe um boreliano $J$-positivo $C \subseteq B$ tal que $C \subseteq A$ ou $C \cap A=\emptyset$.

Antes de decidirmos qual noção de mensurabilidade usaremos, vamos introduzir a definição do ideal $\mathcal{N}_{J}$ e provar algumas afirmações importantes sobre esse ideal.

Definição 5.1.11. Seja $J$ um $\sigma$-ideal Borel gerado. Definimos $\mathcal{N}_{J}$ o ideal auxiliar

$$
\mathcal{N}_{J}=\left\{A \subseteq \omega^{\omega} \mid \forall B \in \mathbb{P}_{J} \exists C \leq B(C \cap A=\emptyset)\right\} .
$$

Segue imediatamente dessa definição que se $A \in \mathcal{N}_{J}$, então o conjunto

$$
D=\left\{B \in \mathbb{P}_{J} \mid B \cap A=\emptyset\right\}
$$

é denso. Por isso, esse ideal auxiliará os argumentos de densidade nos nossos forcings.

Lema 5.1.12. Seja $J$ um $\sigma$-ideal Borel gerado tal que $\mathbb{P}_{J}$ é forcing próprio. Então

1. $\mathcal{N}_{J}$ é um $\sigma$-ideal que estende $J$ e coincide com $J$ nos borelianos.

2. Se $\mathbb{P}_{J}$ tem ccc, então $\mathcal{N}_{J}=J$.

Demonstração. 
1. Seja $A \in J$. Como $J$ é Borel gerado, existe um boreliano $A^{\prime} \in J$ tal que $A \subseteq A^{\prime}$. Agora, se $B \in \mathbb{P}_{J}$, então $B \backslash A^{\prime} \in \mathbb{P}_{J}$ e, portanto, podemos tomar $C=B \backslash A^{\prime}$, e então $C \cap A=\emptyset$.

Agora, se $B \notin J$ é um boreliano (i.e., $B \in \mathbb{P}_{J}$ ), trivialmente não pode existir $C \leq B$ tal que $C \cap B=\emptyset$.

Vamos mostrar que $\mathcal{N}_{J}$ é um $\sigma$-ideal: sejam $\left\{A_{n} \mid n<\omega\right\}$ uma família em $\mathcal{N}_{J}$ e $A=\bigcup_{n<\omega} A_{n}$. Agora, para cada $n<\omega$, defina $D_{n}=\left\{B \in \mathbb{P}_{J} \mid B \cap A_{n}=\emptyset\right\}$. Temos que cada $D_{n}$ é denso. Sejam $B \in \mathbb{P}_{J}$ e $M$ um submodelo elementar enumerável contendo $B$ e $D_{n}$, para todo $n<\omega$, e considere $C=\{x \in B \mid x$ é $M$-genérico $\}$. Temos que, pelo Teorema 5.1.6, $C \in \mathbb{P}_{J}$ e, além disso, para todo $x \in C$ e para todo $n<\omega, x \in \bigcup\left(D_{n} \cap M\right)$. Assim, para cada $n<\omega$, existe $B_{n} \in M$ tal que $B_{n} \cap A_{n}=\emptyset$ e $x \in B_{n}$, o que implica $x \notin A_{n}$. Segue que $C \cap A=\emptyset$ e então $A \in \mathcal{N}_{J}$.

2. Seja $A \in \mathcal{N}_{J}$ e considere o conjunto denso $D=\left\{B \in \mathbb{P}_{J} \mid B \cap A=\emptyset\right\}$. Como $D$ é denso, existe uma anticadeia maximal $E$ tal que $E \subseteq D$. Como $\mathbb{P}_{J}$ tem ccc, $E$ é enumerável, então $C \doteq \omega^{\omega} \backslash \bigcup E$ é boreliano (para isso que precisamos que $E$ fosse enumerável) e $A \subseteq C$. Note que $C \in J$ pois, caso contrário, $E^{\prime}=E \cup\{C\}$ seria uma anticadeia (de fato, $C \cap B=\emptyset$ para todo $B \in E)$.

Teorema 5.1.13. Sejam $J$ um $\sigma$-ideal tal que $\mathbb{P}_{J}$ tem ccc e $A \subseteq \omega^{\omega}$. São equivalentes:

1. Existe um boreliano $B$ tal que $A \Delta B \in J$.

2. Para todo boreliano $J$-positivo $B$, existe um boreliano $J$-positivo $C \subseteq B$ tal que $C \subseteq A$ ou $C \cap A=\emptyset$.

\section{Demonstração.}

- $\left(1 . \Rightarrow 2\right.$.) Seja $B$ um boreliano $J$-positivo qualquer. Considere $B^{\prime}$ boreliano tal que $A \Delta B^{\prime} \in$ $J \subseteq \mathcal{N}_{J}$, pelo Lema 5.1.12. Assim, pela definição de $\mathcal{N}_{J}$, existe $C \subseteq B$ um boreliano $J$-positivo tal que $C \cap\left(A \Delta B^{\prime}\right)=\emptyset$. Temos dois casos: ou $C \cap(A \cup B)=\emptyset$, o que implica $C \cap A=\emptyset$, ou $C \subseteq A \cap B$, o que implica $C \subseteq A$. Note que para essa implicação não usamos que $\mathbb{P}$ tem ccc.

- $(2 . \Rightarrow 1$.) Vamos encontrar um boreliano $B$ que aproxima $A$ "módulo $J$ " por borelianos $J$ positivos dentro de $A$ e fora de $A$. Esse argumento é análogo ao argumento clássico usado para provar que todo conjunto Lebesgue mensurável pode ser aproximado por um $G_{\delta}$ por dentro e um $F_{\sigma}$ por fora. Assim, note que, por hipótese, o conjunto

$$
D \doteq\left\{C \in \mathbb{P}_{J} \mid C \cap A=\emptyset \vee C \subseteq A\right\}
$$

é denso. Seja então $E \subseteq D$ uma anticadeia maximal. Considere $G \doteq \bigcup\{C \subseteq A \mid C \in A\}$ e $F \doteq\left\{C \subseteq \omega^{\omega} \backslash A \mid C \in E\right\}$. Como $\mathbb{P}_{J}$ tem ccc, $E$ é enumerável e, portanto, $G$ e $F$ são borelianos. Além disso, é fácil ver que $A \Delta G=A \backslash G$. Vamos mostrar que $F \backslash G \in J$, e então $G$ pode ser tomado como nosso conjunto $B$ do enunciado. De fato, se $F \backslash G \notin J$, então $F \backslash G$ é um boreliano $J$-positivo, e como $D$ é denso, existe $C \subseteq F \backslash G$ tal que $C \subseteq A$ ou $C \cap A=\emptyset$. Em qualquer um dos casos, $E \cup\{C\}$ é uma anticadeia, o que contraria a maximalidade de $E$. Logo, $F \backslash G \in J$.

Com isso, concluímos que a propriedade do item 1 anterior é mais forte, pois implica na propriedade 2 sem usar a propriedade ccc, e portanto é a candidata natural a ocupar a definição de $\mathbb{P}_{J}$-mensurabilidade. No entanto, os argumentos com a propriedade mais fraca 2 são mais fáceis de serem feitos, e é neste ponto que entra a propriedade ccc: podemos usar a propriedade 2 para definir 
$\mathbb{P}_{J}$-mensurabilidade, pois nos casos em que $\mathbb{P}_{J}$ tem ccc, ela implica na propriedade 1 , como é o caso do forcing aleatório e do forcing de Cohen, que caracterizam a Lebesgue mensurabilidade e a Baire mensurabilidade, respectivamente.

Definição 5.1.14. Seja $J$ um $\sigma$-ideal Borel gerado tal que $\mathbb{P}_{J}$ é forcing próprio. $A \subseteq \omega^{\omega}$ é dito $\mathbb{P}_{J}$-mensurável se, e somente se, para todo $B \in \mathbb{P}_{J}$ existe $C \leq B$ tal que $C \subseteq A$ ou $C \cap A=\emptyset$.

No capítulo anterior, diversas vezes usamos alguma dicotomia para provar alguma noção de mensurabilidade.

Teorema 5.1.15. Sejam $J$ um $\sigma$-ideal Borel gerado e $A \subseteq \omega^{\omega}$. Se A satisfaz a $\mathbb{P}_{J}$-dicotomia então $A$ é $\mathbb{P}_{J}$-mensurável.

Demonstração. Seja $B \in \mathbb{P}_{J}$. Como $A$ satisfaz a $\mathbb{P}_{J}$ dicotomia, então ou existe $C \in \mathbb{P}_{J}$ tal que $A \subseteq C$ (e então $A$ é $\mathbb{P}_{J}$-mensurável, por definição), ou $A \in J \subseteq \mathcal{N}_{J}$ e, neste caso, existe $C \leq B$ tal que $C \cap A=\emptyset$.

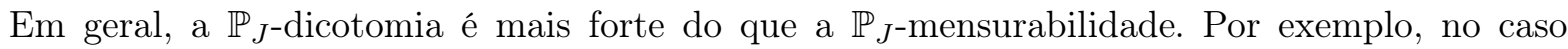
do forcing $\mathbb{P}_{\text {ctbl }}$ (equivalmente $\mathbb{S}$ ), a $\mathbb{P}_{\text {ctbl-mensurabilidade (que é a Marczewski mensurabilidade) }}$ para os conjuntos coanalíticos vale em ZFC, enquanto a $\mathbb{P}_{\text {ctbl-dicotomia (que é a propriedade do }}$ conjunto perfeito) para os conjuntos coanalíticos é equiconsistente com a existência de um cardinal inacessível. Existe, porém, uma condição suficiente para que essas duas noções coincidam:

Teorema 5.1.16. Sejam $J$ um $\sigma$-ideal tal que $\mathbb{P}_{J}$ tem ccc e $A \subseteq \omega^{\omega}$. Então A satisfaz a $\mathbb{P}_{J^{-}}$ dicotomia se, e somente se, $A$ é $\mathbb{P}_{J}$-mensurável.

Demonstração. Já sabemos que se $A$ satisfaz a $\mathbb{P}_{J}$-dicotomia, então $A$ é $\mathbb{P}_{J}$-mensurável. Agora suponha que $A$ é $\mathbb{P}_{J}$-mensurável. Se $A \in J$, acabou. Se $A \notin J$, então $A \notin \mathcal{N}_{J}$ (pelo Lema 5.1.12), e isso implica que existe $B \in \mathbb{P}_{J}$ tal que para todo $C \leq B, C \cap A \neq \emptyset$. Mas como $A$ é $\mathbb{P}_{J}$-mensurável, a última afirmação implica que deve existir algum $C \leq B$ tal que $C \subseteq A$. Logo, $A$ satisfaz a $\mathbb{P}_{J}$-dicotomia.

\subsection{Mensurabilidade obtida colapsando um cardinal inacessível}

Nesta seção descreveremos o Colapso de Levy, que permite construir um modelo de ZFC no qual todo conjunto projetivo de reais é mensurável, para nossa definição mais geral de mensurabilidade. Também é possível definir um modelo interno a esse modelo, que satisfaz ZF + DC e é tal que todo conjunto de reais é mensurável. Levy em 1963 [Lev63], pouco depois de Cohen ter publicado sobre o método do forcing, introduziu uma noção de forcing que colapsa um cardinal inacessível para $\omega_{1}$. A seguir, discutiremos algumas propriedades desse colapso que serão utilizadas para provar o Teorema de Solovay. Toda a discussão relativa feita aqui pode ser encontrada em [Kan08], apenas algumas alterações de caráter organizacional foram feitas. Também foram adicionados alguns detalhes e alguma heurística própria às demonstrações dos teoremas.

Definição 5.2.1. Sejam $S \subseteq \mathrm{ON}$ e $\lambda$ um cardinal regular. $\operatorname{Col}(\lambda, S)$ é o conjunto das condições $p$ que são funções tais que:

- $|p|<\lambda$;

- $\operatorname{dom}(p) \subseteq S \times \lambda$;

- $\forall(\alpha, \xi) \in(S, \lambda)((\alpha, \xi) \in \operatorname{dom}(p) \rightarrow p(\alpha, \xi)<\alpha)$; 
onde a ordem é a de inclusão contrária $\supseteq$. Quando $S=\kappa>\lambda$ e $\operatorname{Col}(\lambda, \kappa)$ tem $\kappa$-c.c, dizemos que $\operatorname{Col}(\lambda, \kappa)$ é o Colapso de Levy de $\kappa$ para $\lambda^{+}$. Veremos a seguir por quê.

Faremos a análise do Colapso de Levy para o caso geral $S \subseteq$ ON, mas estamos interessados principalmente no caso em que $S=\kappa$ é um cardinal inacessível. A primeira observação é que o Colapso de Levy colapsa um cardinal $\kappa$ inacessível para $\lambda^{+}$na extensão genérica.

Teorema 5.2.2. Sejam $\kappa>\lambda, \lambda, \kappa$ regulares. Se $\lambda=\omega$ ou se $\kappa$ é inacessível então

1. $\operatorname{Col}(\lambda, \kappa)$ é $\lambda$-fechado.

2. $\operatorname{Col}(\lambda, \kappa)$ tem $\kappa$-c.c.

3. Forçando $\operatorname{com} \operatorname{Col}(\lambda, \kappa): \Vdash \check{\kappa}=\check{\lambda}^{+}$.

\section{Demonstração.}

1. Para este item poderíamos trocar $\kappa$ por $S \subseteq$ ON. Sejam então $\delta<\lambda$ e $\left\{p_{\gamma} \mid \gamma<\delta\right\} \subseteq$ $\operatorname{Col}(\lambda, \kappa)$ uma $\delta$-sequência decrescente (o que é uma $\delta$-sequência $\subseteq$-crescente). Seja então $p \doteq \bigcup\left\{p_{\gamma} \mid \gamma<\delta\right\}$. Temos que $p$ é uma função pois é a união de uma cadeia e $|p|<\lambda$ pois $\lambda$ é regular. Segue que $p \in \operatorname{Col}(\lambda, \kappa)$.

2. Considere $\left\{p_{\alpha} \mid \alpha<\kappa\right\} \subseteq \operatorname{Col}(\lambda, \kappa)$. Vamos mostrar que esse conjunto não pode ser uma anticadeia, exibindo condições compatíveis. Note que nos casos em que $\lambda=\omega$ ou que $\kappa$ é inacessível, vale que se $\alpha<\kappa$ então $\alpha^{<\lambda}<\kappa$. Além disso, todas as nossas condições têm cardinalidade $<\lambda$. Portanto, podemos aplicar o Lema do $\Delta$-sistema para $\left\{\operatorname{dom}\left(p_{\alpha}\right) \mid \alpha<\kappa\right\}$ obtendo um $X \in[\kappa]^{\kappa}$ tal que $\left\{\operatorname{dom}\left(p_{\alpha}\right) \mid \alpha \in X\right\}$ é um $\Delta$-sistema. Isto é, existe algum $r \subseteq \kappa \times \lambda$ tal que $\operatorname{dom}\left(p_{\alpha}\right) \cap \operatorname{dom}\left(p_{\beta}\right)=r$, sempre que $\alpha \neq \beta$ estão ambos em $X$. Agora estamos interessados em saber quantas funções com domínio $r$ estão em $\left\{p_{\alpha} \mid \alpha \in X\right\}$. Note que $|r|<\lambda<\kappa$, e se $\delta \doteq \sup \{\alpha<\lambda \mid \exists \xi((\alpha, \xi) \in r)\} \leq \lambda<\kappa$, e se $p_{\beta}$ tem domínio $r$, então $p_{\beta}(\alpha, \xi)<\delta$, para qualquer $(\alpha, \xi) \in r$. Assim, todas as funções em $\left\{p_{\alpha} \mid \alpha \in X\right\}$ com domínio $r$ têm imagem contida em $\delta$, e como $\left|\delta^{r}\right|<\kappa$, então deve existir também $Y \in[X]^{\kappa}$ tal que $p_{\alpha}\left\lceil r=p_{\beta}\left\lceil r\right.\right.$ sempre que $\alpha, \beta \in Y, \alpha \neq \beta$ e para tais $\alpha, \beta, p_{\alpha}, p_{\beta}$ são compatíveis.

3. Seja $G$ um filtro $\operatorname{Col}(\lambda, \kappa)$-genérico. Temos que $f_{G} \doteq \bigcup G$ é uma função de $\kappa \times \lambda$ em $\kappa$. Agora, se $\alpha<\kappa$, então a função $f_{G}^{\alpha}: \lambda \rightarrow \alpha$ definida por $f_{G}^{\alpha}(\beta)=f_{G}(\alpha, \beta)$ é uma sobrejeção. Logo, em $\mathrm{V}[G]|=| \alpha \mid=\lambda$ e, $\operatorname{como} \operatorname{Col}(\lambda, \kappa)$ preserva cardinais $\leq \lambda$ e $\geq \kappa$, então $\mathrm{V}[G] \models \kappa=\lambda^{+}$.

Agora suponha que $\kappa$ é um cardinal inacessível e considere $\operatorname{Conl}(\omega, \kappa)$ o Colapso de Levy. Seja $G$ um filtro $\operatorname{Col}(\omega, \kappa)$-genérico. Nossa intenção é obter a $\mathbb{P}_{J \text {-mensurabilidade para uma certa }}$ classe de conjuntos em $\mathrm{V}[G]$. Uma propriedade-chave dessa extensão genérica é que para quaisquer sequências de ordinais $s_{1}, s_{2} \in \mathrm{V}[G]$ e qualquer fórmula $\varphi\left(v_{1}, v_{2}\right)$, podemos "fatorar" o modelo $\mathrm{V}[G]$ em $V\left[s_{1}\right]\left[s_{2}\right]$ de modo que, para alguma fórmula correspondente $\tilde{\varphi}\left(v_{1}, v_{2}\right)$,

$$
\mathrm{V}[G] \models \varphi\left(s_{1}, s_{2}\right) \leftrightarrow V\left[s_{1}\right]\left[s_{2}\right] \models \tilde{\varphi}\left(s_{1}, s_{1}\right) .
$$

Logo a importância dessa propriedade do Colapso de Levy ficará clara. Os lemas a seguir servem para provar essa propriedade.

Lema 5.2.3. Sejam $S \subseteq \mathrm{ON}, S=X \cup Y$ uma união disjunta, $\lambda$ regular, $\mathbb{P}_{0}=\operatorname{Col}(\lambda, X) e$ $\mathbb{P}_{1}=\operatorname{Col}(\lambda, Y)$. Então $G$ é $\operatorname{Col}(\lambda, S)$-genérico se, e somente se, $G=\left\{p \cup q:(p, q) \in G_{0} \times G_{1}\right\}$, onde $G_{0}$ é $\mathbb{P}_{0}$-genérico, $\operatorname{Col}(\lambda, Y)^{V\left[G_{0}\right]}=\mathbb{P}_{1}$ e $G_{1}$ é $\mathbb{P}_{1}$-genérico sobre $V\left[G_{0}\right]$. 
Demonstração. Note que a aplicação $p \mapsto(p \nmid X \times \lambda, p \nmid Y \times \lambda)$ é um isomorfismo de $\operatorname{Col}(\lambda, S)$ em $\mathbb{P}_{0} \times \mathbb{P}_{1}$, e podemos usar o resultado para o forcing produto. Além disso, se $f \in \operatorname{Col}(\lambda, Y) \cap V\left[G_{0}\right]$ então $f \in \operatorname{Col}(\lambda, Y)$ pois o forcing é $\lambda$-fechado, e portanto não adiciona sequências de tamanho menor que $\lambda$.

Lema 5.2.4. Sejam $\kappa>\lambda, \lambda, \kappa$ regulares tais que $\operatorname{Col}(\lambda, \kappa)$ tem $\kappa$-c.c., $G$ um filtro $\operatorname{Col}(\lambda, \kappa)$ genérico e $s \in \mathrm{V}[G]$, $s: \gamma \rightarrow$ ON uma $\gamma$-sequência de ordinais com $\gamma<\kappa$. Então existe $\delta<\kappa$ tal que $s \in V[G \cap \operatorname{Col}(\lambda, \delta)]$.

Demonstração. Queremos achar algum $\delta<\kappa$ de modo que $s: \gamma \rightarrow$ ON possa ser definida a partir de $G \cap \operatorname{Col}(\lambda, \delta)$. Assim, como $s: \gamma \rightarrow$ ON é uma $\gamma$-sequência em $\mathrm{V}[G]$, então para cada $\alpha<\gamma$, existem $p \in G$ e $\xi \in \mathrm{ON}$ tais que $p \Vdash \dot{s}(\check{\alpha})=\check{\xi}$. Queremos que essa condição $p$ tenha sempre domínio contido em algum $\delta \times \lambda$. Considere então $X_{\alpha}=\{p \in \operatorname{Col}(\lambda, \kappa) \mid \exists \xi \in \mathrm{ON}(p \Vdash \dot{s}(\check{\alpha})=\check{\xi})\}$. Pelo comentário anterior, $X_{\alpha} \neq \emptyset$. Agora, os $X_{\alpha}$ são os conjuntos de condições que forçam os valores da sequência de ordinais que fixamos, mas pode ter muitas dessas condições. Além disso, queremos que essas condições estejam em algum colapso de cardinalidade menor, algum $\operatorname{Col}(\omega, \delta)$. Para isso, podemos usar a condição de anticadeia do colapso, que limita os tamanhos das condições. Seja então uma anticadeia maximal $A_{\alpha} \subseteq X_{\alpha}$. $\operatorname{Como} \operatorname{Col}(\lambda, \kappa)$ tem $\kappa$-c.c., $\left|A_{\alpha}\right|<\kappa$ e, como $\kappa$ é regular, $\left|\bigcup_{\alpha<\gamma} A_{\alpha}\right|<\kappa$. Logo, existe algum $\delta<\kappa$ tal que sempre que $p \in \bigcup_{\alpha<\gamma} A_{\alpha}$ tem-se $\operatorname{dom}(p) \subseteq \delta \times \lambda$. Agora, podemos definir $s$ a partir de $G \cap \operatorname{Col}(\lambda, \delta)$ da seguinte forma:

$$
s(\alpha)=\xi \leftrightarrow p \Vdash \dot{s}(\check{\alpha})=\check{\xi},
$$

onde $p$ é o único elemento de $G \cap A_{\alpha}$.

Lema 5.2.5. Seja $\mathbb{P}$ uma noção de forcing separativa tal que $|\mathbb{P}| \leq|\alpha|$ e

$$
\Vdash \exists f(f: \check{\omega} \rightarrow \check{\alpha} \text { é sobrejetora } \wedge f \notin \check{V}) .
$$

Então existe um mergulho denso injetor de um subconjunto denso de $\operatorname{Col}(\omega,\{\alpha\})$ em $\mathbb{P}$, e portanto $\operatorname{Col}(\omega,\{\alpha\})$ e $\mathbb{P}$ são forcing-equivalentes.

Demonstração. Primeiro, fixemos $\nu=|\alpha|$. Vamos mostrar que, para qualquer $p \in \mathbb{P}$, existe uma anticadeia maximal abaixo de $p$ de cardinalidade $\nu$. Se $\nu=\aleph_{0}$, isso ocorre pois, caso contrário, teríamos que $\mathbb{P}$ seria um filtro $\mathbb{P}$-genérico, e então não teríamos que $\Vdash f \notin \check{V}$. Se $\nu>\aleph_{0}$, isso ocorre pois o forcing colapsa $\nu$ para $\omega$, então não vale $\nu$-c.c.

Agora, o subconjunto denso de $\operatorname{Col}(\omega,\{\alpha\})$ que queremos é

$$
D=\{p \in \operatorname{Col}(\omega,\{\alpha\}) \mid \exists n(p:\{\alpha\} \times n \rightarrow \alpha)\} .
$$

Note que o colapso de $\nu$ para $\aleph_{0}$ implica que a cardinalidade do filtro genérico também é colapsada para $\aleph_{0}$, uma vez que $|\mathbb{P}| \leq \nu$ e $G \subseteq \mathbb{P}$ na extensão genérica. Assim, $\Vdash \exists g(g: \omega \rightarrow$ $\dot{G}$ é sobrejetora ) e, pelo princípio maximal, existe um $\mathbb{P}$-nome $\dot{g}$ tal que $\Vdash \dot{g}: \check{\omega} \rightarrow \dot{G}$ é sobrejetora .

Agora, construiremos um isomorfismo $e: D \rightarrow e[D]$ e mostraremos que $e[D]$ é denso em $\mathbb{P}$.

Defina $e(\emptyset)=\mathbb{1}$. Suponha que definimos $e(p)$, com $p \in D, p:\{\alpha\} \times n \rightarrow \alpha$. Cuidaremos agora de todos as condições em $D$ que têm cardinalidade $n+1$. Assim, considere $\left\{a_{\xi}^{p}: \xi<\alpha\right\}$ uma anticadeia maximal abaixo de $e(p)$ de cardinalidade $\nu$. Podemos assumir que $a_{\xi}^{p} \Vdash \dot{g}(\check{n})=\check{r}$, para algum $r \in \mathbb{P}$. Para cada $\xi<\alpha$ defina $e(p \cup\{((\alpha, n), \xi)\})=a_{\xi}^{p}$. Note que $e: D \rightarrow e[D]$ assim definido é um isomorfismo. Agora vamos mostrar que $e[D]$ é denso em $\mathbb{P}$.

Seja então $r \in \mathbb{P}$. Como $r \Vdash \check{r} \in \dot{G}$, então existem $s \leq r$ e $n \in \omega$ tais que $s \Vdash \dot{g}(\check{n})=\check{r}$. Além disso, por construção temos que existe $p \in D$ tal que $|p|=n+1$ e $e(p) \| s$. Como $e(p) \Vdash \dot{g}(\check{n})=\check{t}$ para algum $t \in \mathbb{P}$, temos que $e(p) \Vdash \dot{g}(\check{n})=\check{r}$ e, portanto, $e(p) \Vdash \check{r} \in \dot{G}$. Logo, pela separatividade, $e(p) \leq r$. 
Lema 5.2.6. Suponha que $\kappa>\omega$ é regular e $G$ é $\operatorname{Col}(\omega, \kappa)$-genérico. Então para todo $s \in \mathrm{V}[G]$ com $s: \omega \rightarrow \mathrm{ON}$, existe $H$ que é $\mathrm{Col}(\omega, \kappa)$-genérico sobre $V[s]$ tal que

$$
\mathrm{V}[G]=V[s][H] .
$$

Demonstração. Pelo Lema 5.2.4, existe $\delta<\kappa$ tal que $s \in V[G \cap \operatorname{Col}(\omega, \delta)]$. Vamos particionar o filtro genérico em duas partes, e então em três, usando o Lema 5.2.3. Note que $\kappa=\kappa \backslash(\delta+1) \cup(\delta+1)$, e $\delta+1=\delta \cup\{\delta\}$, sendo essas uniões disjuntas. Agora, considere os seguintes filtros:

$$
\begin{aligned}
& G_{0} \doteq G \cap \operatorname{Col}(\omega, \delta) \\
& G_{1} \doteq G \cap \operatorname{Col}(\omega,\{\delta\}) \\
& G_{2} \doteq G \cap \operatorname{Col}(\omega, \kappa \backslash(\delta+1))
\end{aligned}
$$

Primeiro, pelo Lema 5.2.3, $G_{0}$ é $\operatorname{Col}(\omega, \delta)$-genérico, e como $s \in V\left[G_{0}\right]$, então pelo Teorema 3.3.11

$$
V \subseteq V[s] \subseteq V\left[G_{0}\right]
$$

e além disso, existe uma noção de forcing $\mathbb{P} \in V[s]$, que pelo Teorema 3.2.32 podemos assumir ser separativa, e existe $H_{0}$ um filtro $\mathbb{P}$-genérico sobre $V[s]$ tal que $V\left[G_{0}\right]=V[s]\left[H_{0}\right]$. Agora, argumentando em $V[s]$, considere $\mathbb{Q}=\mathbb{P} \times \operatorname{Col}(\omega,\{\delta\})$. Pelo Lema 5.2.3, $G_{1}$ é $\operatorname{Col}(\omega,\{\delta\})$-genérico sobre $V\left[G_{0}\right]$, e portanto $V[s]\left[H_{0}\right]\left[G_{1}\right]$ é uma extensão genérica sobre $V[s]$ usando o forcing $\mathbb{Q} \in V[s]$. Por outro lado, note que $|\mathbb{Q}| \leq|\operatorname{Col}(\omega, \delta+1)|=|\delta|$ e $\Vdash_{\mathbb{Q}} \exists f(f: \check{\omega} \rightarrow \check{\delta}$ é sobrejetora $\wedge f \notin \check{V})$, pois a segunda componente de $\mathbb{Q}$ - i.e., $\operatorname{Col}(\omega,\{\delta\})$ - colapsa $\delta$ para $\omega$. Pelo Lema 5.2 .5 , existe um mergulho denso injetor de um subconjunto denso de $\operatorname{Col}(\omega,\{\delta\})$ em $\mathbb{Q}$, o que implica que $\mathbb{Q}$ e $\operatorname{Col}(\omega,\{\delta\})$ são forcing-equivalentes. Assim, deve existir $H_{1}$ um filtro $\operatorname{Col}(\omega,\{\delta\})$-genérico sobre $V[s]$ tal que $V[s]\left[H_{1}\right]=V[s]\left[H_{0}\right]\left[G_{1}\right]$. Aplicando o Teorema 5.2.5 também ao forcing $\operatorname{Col}(\omega, \delta+1)$, de forma análoga concluimos que deve existir $H_{2}$ um filtro $\operatorname{Col}(\omega, \delta+1)$-genérico sobre $V[s]$ tal que $V[s]\left[H_{2}\right]=V[s]\left[H_{1}\right]$.

$$
\mathrm{V}[G]=V\left[G_{0}\right]\left[G_{1}\right]\left[G_{2}\right]=V[s]\left[H_{0}\right]\left[G_{1}\right]\left[G_{2}\right]=V[s]\left[H_{2}\right]\left[G_{2}\right]
$$

e $H_{2} \cup G_{2}$ é $\operatorname{Col}(\omega, \kappa)$-genérico sobre $V[s]$.

Lema 5.2.7. Sejam $S \subseteq \mathrm{ON}$ um conjunto de ordinais e $\lambda$ um cardinal regular. Então $\operatorname{Col}(\lambda, S)$ é fracamente homogêneo.

Demonstração. Sejam $p, q \in \operatorname{Col}(\lambda, S)$. A fim de que um automorfismo $e \operatorname{de} \operatorname{Col}(\lambda, S)$ seja tal que $e(p) \| q$ é suficiente que $\operatorname{dom}(e(p)) \cap \operatorname{dom}(q)=\emptyset$. Considere $f: \lambda \rightarrow \lambda$ uma bijeção tal que $f(\xi) \neq \zeta$ sempre que $(\alpha, \xi) \in \operatorname{dom}(p)$ e $(\beta, \zeta) \in \operatorname{dom}(q)$. Usaremos essa função para induzir um automorfismo $e$, da seguinte forma: para cada $r \in \operatorname{Col}(\lambda, S), e(r) \in \operatorname{Col}(\lambda, S)$ é tal que

$$
(\alpha, \xi) \in \operatorname{dom}(e(r)) \leftrightarrow(\alpha, f(\xi)) \in \operatorname{dom}(r) \wedge e(r)(\alpha, \xi)=r(\alpha, f(\xi)) .
$$

Note que $\operatorname{dom}(e(p)) \cap \operatorname{dom}(q)=\emptyset$. De fato, se tivéssemos $(\alpha, \xi) \in \operatorname{dom}(e(p)) \cap \operatorname{dom}(q)$, então teríamos $(\alpha, f(\xi)) \in \operatorname{dom}(p)$ e $(\alpha, \xi) \in \operatorname{dom}(q)$, mas pela nossa escolha de $f$, isso implicaria $f(\xi) \neq$ $f(\xi)$, absurdo.

Teorema 5.2.8. Suponha que $\kappa>\omega$ é regular e $G$ é $\operatorname{Col}(\omega, \kappa)$-genérico. Então para qualquer fórmula $\varphi(v)$ existe uma fórmula $\tilde{\varphi}(v)$ tal que para qualquer $s \in \mathrm{ON}^{\omega} \cap \mathrm{V}[G]$,

$$
\mathrm{V}[G] \models \varphi(s) \leftrightarrow V[s] \models \tilde{\varphi}(s) .
$$

Demonstração. Pelo Lema 5.2.6, existe $H$ um filtro $\operatorname{Col}(\omega, \kappa)$-genérico sobre $V[s]$ tal que $\mathrm{V}[G]=$ 
$V[s][H]$. Assim,

$$
\begin{aligned}
\mathrm{V}[G] \models \varphi(s) & \leftrightarrow V[s][H] \models \varphi(s) \\
& \leftrightarrow \exists p \in H(p \Vdash \varphi(\check{s})) \\
& \stackrel{*}{\leftrightarrow} V[s] \models(\Vdash \varphi(\check{s}))
\end{aligned}
$$

onde $(*)$ segue da Lei 0-1 para forcings fracamente homogêneos (Teorema 3.2.34). Assim, podemos tomar a fórmula $\tilde{\varphi}(s)$ como sendo $\Vdash \varphi(\check{s})$.

Usaremos uma versão do teorema anterior para fórmulas de duas variáveis. Para provar essa versão do teorema, é preciso fazer uma alteração equivalente no Lema 5.2.6. A complicação é somente notacional.

Antes de provar o teorema principal dessa seção, precisamos garantir que a condição de inacessibilidade seja preservada por forcing.

Teorema 5.2.9. Sejam $\kappa$ um cardinal inacessivel e $\mathbb{P}$ é uma noção de forcing tal que $|\mathbb{P}|<\kappa$. Então

$$
\Vdash \kappa \text { é inacessivel. }
$$

Demonstração. Como $\mathbb{P}$ tem $|\mathbb{P}|^{+}$-c.c, então $\Vdash \kappa$ é regular. Além disso, se $\lambda$ é um cardinal qualquer, então

$$
\Vdash 2^{\lambda} \leq\left(|\mathbb{P}|^{<\kappa}\right)^{\lambda},
$$

o que implica que $\Vdash \kappa$ é limite forte.

Teorema 5.2.10. Sejam $\kappa$ um cardinal inacessivel, $G$ um filtro $\operatorname{Col}(\omega, \kappa)$-genérico e $J$ um $\sigma$-ideal Borel gerado tal que $\mathbb{P}_{J}$ é forcing próprio. Então em $\mathrm{V}[G]$ todos os conjuntos sequencialmente ordinal definíveis são $\mathbb{P}_{J}$-mensuráveis.

Demonstração. Em $\mathrm{V}[G]$ considere $A$ um conjunto definível por uma sequência enumerável de ordinais. Isto é, sejam $s \in \mathrm{ON}^{\omega} \cap \mathrm{V}[\mathrm{G}]$ e $\varphi\left(v_{1}, v_{2}\right)$ uma fórmula tais que

$$
\mathrm{V}[G] \models x \in A \leftrightarrow \mathrm{V}[G] \models \varphi(s, x) .
$$

Agora, pelo Teorema 5.2.8, existe uma fórmula $\widetilde{\varphi}\left(v_{1}, v_{2}\right)$ tal que, para todo $x \in A$ :

$$
\mathrm{V}[G] \models \varphi(s, x) \leftrightarrow V[s][x] \models \widetilde{\varphi}(s, x) .
$$

Seja $B \in \mathbb{P}_{J}^{\mathrm{V}[G]}$ um boreliano $J$-positivo codificado em $\mathrm{V}[G]$. Podemos assumir sem perda de generalidade que o código de $B$ está contido em $s$. Considere o forcing $\mathbb{P}_{J}$ em $V[s]: \mathbb{P}_{J}^{V[s]}$. Assim, fixe $B^{\prime} \leq B, B^{\prime}$ codificado em $V[s]$ tal que $\left(B^{\prime} \Vdash \widetilde{\varphi}\left(\check{s}, \dot{x}_{G}\right)\right)^{V[s]}$ ou $\left(B^{\prime} \Vdash \neg \widetilde{\varphi}\left(\check{s}, \dot{x}_{G}\right)\right)^{V[s]}$. Como $\kappa$ é inacessível em $V[s]$, então

$$
\left(\mid\left\{D \subseteq \mathbb{P}_{J} \mid D \text { é denso }\right\} \mid<\kappa\right)^{V[s]} .
$$

Assim, em $\mathrm{V}[G]$, a família dos densos em $\mathbb{P}_{J}^{V[s]}$ é enumerável, pois $\kappa=\aleph_{1}^{\mathrm{V}[G]}$. Seja $\left\{D_{n} \mid n<\omega\right\} \in$ $\mathrm{V}[G]$ uma enumeração dos densos de $\mathbb{P}_{J}^{V[s]}$. Considere o conjunto

$$
X=\left\{x \in \omega^{\omega} \mid x \text { não é } \mathbb{P}_{J} \text {-genérico sobre } V[s]\right\} .
$$

Vamos mostrar que $X \in \mathcal{N}_{J}$. Primeiro note que

$$
x \in X \leftrightarrow x \in \omega^{\omega} \backslash \bigcup D_{n}, \text { para algum } n
$$

Assim, se definirmos $A_{n} \doteq \omega^{\omega} \backslash \bigcup D_{n}$, para todo $n<\omega$, temos que $X=\bigcup_{n<\omega} A_{n}$. Logo, basta mostrar que cada $A_{n} \in \mathcal{N}_{J}$ e usar que $\mathcal{N}_{J}$ é um $\sigma$-ideal. De fato, seja $E \in \mathbb{P}_{J}$ qualquer. Como cada $D_{n}$ é denso, existe $C_{n} \in D_{n}$ tal que $C_{n} \leq E$, e então $C_{n} \cap A_{n}=\emptyset$. Segue que $A_{n} \in \mathcal{N}_{J}$. Portanto, 
existe $C \leq B^{\prime}$ um boreliano $J$-positivo codificado em $\mathrm{V}[G]$ tal que todo $x \in C$ é $\mathbb{P}_{J}$-genérico sobre $V[s]$. Fixe $x \in C$.

- Se $\left(B^{\prime} \Vdash \widetilde{\varphi}\left(\check{s}, \dot{x}_{G}\right)\right)^{V[s]}$ então $V[s][x] \models \widetilde{\varphi}(s, x)$, o que implica $\mathrm{V}[G] \models \varphi(s, x)$ e, portanto, $\mathrm{V}[G] \models x \in A$. Segue que $\mathrm{V}[G] \models C \subseteq A$.

- Se $\left(B^{\prime} \Vdash \neg \widetilde{\varphi}\left(\check{s}, \dot{x}_{G}\right)\right)^{V[s]}$ então $V[s][x] \models \neg \widetilde{\varphi}(s, x)$, o que implica $\mathrm{V}[G] \models \neg \varphi(s, x)$ e, portanto, $\mathrm{V}[G] \models x \notin A$. Segue que $\mathrm{V}[G] \models C \cap A=\emptyset$.

Logo, $\left(A \text { é } \mathbb{P}_{J} \text {-mensurável }\right)^{\mathrm{V}[G]}$.

Corolário 5.2.11 (Teorema de Solovay). Seja $J$ um $\sigma$-ideal Borel gerado tal que $\mathbb{P}_{J}$ é forcing próprio. Se existe um cardinal inacessivel então

1. Existe um modelo de ZFC onde todos os conjuntos projetivos são $\mathbb{P}_{J}$-mensuráveis.

2. Existe um modelo de $\mathrm{ZF}+\mathrm{DC}$ onde todos os conjuntos de reais são $\mathbb{P}_{J}$-mensuráveis.

Demonstração.

1. Basta observar que todo conjunto projetivo é sequencialmente ordinal definível. Então no modelo $\mathrm{V}[G]$ todos os conjuntos projetivos são $\mathbb{P}_{J}$-mensuráveis.

2. Basta considerar o modelo $\mathrm{HOD}^{\mathrm{V}[\mathrm{G}]}$, que é o modelo interno $\mathrm{HOD}^{\omega}$ definido dentro de $\mathrm{V}[G]$. Esse modelo satisfaz ZF + DC e, além disso, todo conjunto de reais é sequencialmente ordinal definível.

Embora seja um resultado conhecido que a propriedade do conjunto perfeito seja verdadeira no Modelo de Solovay, assim como as propriedades de dicotomia abordadas no capítulo anterior, não se conhece uma prova uniforme para a $\mathbb{P}_{J}$-dicotomia, nem algum contraexemplo.

Problema em aberto 1. Seja $J$ um $\sigma$-ideal Borel gerado tal que $\mathbb{P}_{J}$ é forcing próprio. Todos os suconjuntos dos reais satisfazem a $\mathbb{P}_{J}$-dicotomia no Modelo de Solovay?

Como vimos na Seção 2.4, o uso de jogos infinitos e teoremas de determinância para jogos é um dos mainstreams da teoria dos conjuntos. Em geral, técnicas de determinância são usadas para provar a regularidade para diversas classes de conjuntos definíveis. Não se sabe, porém, se AD implica que todo conjunto de números reais é $\mathbb{P}_{J}$-mensurável, ou que satisfaz a $\mathbb{P}_{J}$-dicotomia.

Problema em aberto 2. Se J é uma $\sigma$-ideal Borel gerado tal que $\mathbb{P}_{J}$ é forcing próprio. AD implica que todos os suconjuntos dos reais satisfazem a $\mathbb{P}_{J}$-dicotomia? E que todos eles são $\mathbb{P}_{J}$-mensuráveis?

\subsection{Mensurabilidade dos conjuntos $\Delta_{2}^{1}$ e $\Sigma_{2}^{1}$ via forcing sobre L}

Ao fim da Seção 4.1 foi mencionada a possibilidade de se obter regularidade para os conjuntos projetivos sem o uso de um cardinal inacessível. As caracterizações do tipo Solovay e do tipo Ihoda-Shelah mencionadas ao fim da Seção 4.2.4 podem nos ajudar com esse problema. Com essas caracterizações, o problema de obter regularidade para os conjuntos $\boldsymbol{\Delta}_{2}^{1}$ e $\boldsymbol{\Sigma}_{2}^{1}$ se transforma no problema de se adicionar, usando forcing iterado a partir de L, alguma certa quantidade de reais genéricos (ou quasi-genéricos). Em 1999 [BL99] Brendle e Löwe investigaram caracterizações desse tipo para alguns forcings arbóreos. Destacamos as caracterizações obtidas para os forcings $\mathbb{S}, \mathbb{M}$ e $\mathbb{L}$ :

Teorema 5.3.1 ([BL99]). 
1. As seguintes afirmações são equivalentes:

1a. Todo conjunto $\boldsymbol{\Sigma}_{2}^{1}$ de reais é Marczewski mensurável.

1b. Todo conjunto $\boldsymbol{\Delta}_{2}^{1}$ de reais é Marczewski mensurável.

1c. Para todo real $r$, existe um número real que não está em $\mathrm{L}[r]$.

2. As seguintes afirmações são equivalentes:

2a. Todo conjunto $\boldsymbol{\Sigma}_{2}^{1}$ de reais é Miller mensurável.

2b. Todo conjunto $\boldsymbol{\Delta}_{2}^{1}$ de reais é Miller mensurável.

2c. Para todo real $r$, existe um número real ilimitado sobre $\mathrm{L}[r]$.

3. As seguintes afirmações são equivalentes:

3a. Todo conjunto $\boldsymbol{\Sigma}_{2}^{1}$ de reais é Laver mensurável.

3b. Todo conjunto $\boldsymbol{\Delta}_{2}^{1}$ de reais é Laver mensurável.

3c. Para todo real $r$, existe um número real dominante sobre $\mathrm{L}[r]$.

Note que a caracterização para a mensurabilidade para as noções de forcing acima envolvem reais novos, iliminantes e dominantes, e não reais de Sacks, de Miller e de Laver. Veremos que isso pode ser resolvido introduzindo a noção de real quasi-genérico (assim, por exemplo, um real ilimitado sobre $\mathrm{L}[r]$ será um real $\mathbb{M}$-quasi-genérico sobre $\mathrm{L}[r]$ ).

Nosso objetivo nessa seção é provar alguns resultados sobre a $\mathbb{P}_{J}$-mensurabilidade para os conjuntos $\boldsymbol{\Sigma}_{1}^{1}, \boldsymbol{\Delta}_{2}^{1}$ e $\boldsymbol{\Sigma}_{2}^{1}$. Primeiramente, como esperado, todos os conjuntos $\boldsymbol{\Sigma}_{1}^{1}$ de reais são $\mathbb{P}_{J}$-mensuráveis.

Teorema 5.3.2. Seja $J$ um $\sigma$-ideal Borel gerado tal que $\mathbb{P}_{J}$ é forcing próprio. Então todos os conjuntos analíticos são $\mathbb{P}_{J}$-mensuráveis.

Demonstração. Sejam $A$ um conjunto analítico, $r \in \omega^{\omega}$ um parâmetro real tal que $A$ é definível por uma fórmula $\varphi \in \Sigma_{1}^{1}(r)$. Sejam $B$ um boreliano $J$-positivo e $M$ um submodelo elementar enumerável contendo $r$ e $B$. Em $M$, deve existir $B^{\prime} \leq B$ tal que $\left(B^{\prime} \Vdash \varphi\left(\dot{x}_{G}\right)\right)^{M}$ ou $\left(B^{\prime} \Vdash \neg \varphi\left(\dot{x}_{G}\right)\right)^{M}$. Em qualquer um dos casos, seja $C=\left\{x \in B^{\prime} \mid x\right.$ é $M$-genérico $\}$ a condição $M$-master abaixo de $B^{\prime}$, que provamos ser um boreliano $J$-positivo.

- Se $\left(B^{\prime} \Vdash \varphi\left(\dot{x}_{G}\right)\right)^{M}$, então $C \Vdash \varphi\left(\dot{x}_{G}\right)$ e, portanto, para todo $x \in C, M[x] \models \varphi(x)$. Como $\varphi$ é uma fórmula $\Sigma_{1}^{1}$, então por absolutividade analítica, $\mathrm{V} \models \varphi(x)$. Assim, $C \subseteq A$.

- Se $\left(B^{\prime} \Vdash \neg \varphi\left(\dot{x}_{G}\right)\right)^{M}$ e, portanto, para todo $x \in C, M[x] \models \neg \varphi(x)$. Como anteriormente, vale $\mathrm{V} \models \neg \varphi(x)$ e, portanto, $C \cap A=\emptyset$.

Assim como não se sabe se todas todas as $\mathbb{P}_{J}$-dicotomias são verdadeiras no modelo de Solovay, também não se sabe se todos os conjuntos analíticos satisfazem as $\mathbb{P}_{J}$-dicotomias.

Problema em aberto 3. Seja $J$ um $\sigma$-ideal Borel gerado tal que $\mathbb{P}_{J}$ é forcing próprio. Todo conjunto analítico satisfaz a $\mathbb{P}_{J}$-dicotomia?

Na Seção 1.5 vimos que em L há contraexemplos para a regularidade dos conjuntos projetivos. Não existe exceção para o nosso caso de regularidade idealizada.

Teorema 5.3.3. Seja $J$ um $\sigma$-ideal. Se $\mathrm{V}=\mathrm{L}$, então existe um conjunto $\boldsymbol{\Delta}_{2}^{1}$ que não é $\mathbb{P}_{J^{-}}$ mensurável. 
Demonstração. Primeiramente, note que como os $\sigma$-ideais contém todos os conjuntos enumeráveis, então todos os borelianos $J$-positivos são não-enumeráveis. Como todo boreliano tem a propriedade do conjunto perfeito, então todo boreliano $J$-positivo deve conter um conjunto perfeito. Agora, se $A$ é um conjunto $\mathbb{P}_{J}$-mensurável e $B$ é um boreliano $J$-positivo tal que $B \subseteq A$, então $A$ contém um conjunto perfeito, e se $B \cap A=\emptyset$, então $\omega^{\omega} \backslash A$ contém um conjunto perfeito. Pelo Teorema 1.5.13, existe $A$ um conjunto $\Delta_{2}^{1}$ de Bernstein, que por definição não contém nenhum conjunto perfeito, nem é disjunto de algum conjunto perfeito, e portanto não pode ser $\mathbb{P}_{J}$-mensurável.

Temos uma condição suficientemente boa para que os conjuntos $\boldsymbol{\Delta}_{2}^{1}$ sejam $\mathbb{P}_{J}$-mensuráveis.

Teorema 5.3.4. Se para todo $r \in \omega^{\omega}$ existe um real $\mathbb{P}_{J}$-genérico sobre $\mathrm{L}[r]$ então todos os conjuntos $\Delta_{2}^{1}$ de reais são $\mathbb{P}_{J}$-mensuráveis.

Demonstração. Sejam $A$ um conjunto $\Delta_{2}^{1}$ e $r \in \omega^{\omega}$ um parâmetro real tal que $A$ é definível por fórmulas $\varphi \in \Delta_{2}^{1}(r)$. Sejam $B$ um boreliano $J$-positivo cujo código de Borel é $c$ e $x$ o real $\mathbb{P}_{J}$-genérico sobre $\mathrm{L}[r, c]$. Temos que ou $\varphi(x)$ é verdadeira, ou $\neg \varphi(x)$ é verdadeira, e como as duas fórmulas são $\Sigma_{2}^{1}(r)$, então o mesmo ocorre em L $[r, c, x]$, pelo Teorema da Absolutividade de Shoenfield. Assim, deve existir $B^{\prime} \leq B$ em $\mathrm{L}[r, c]$ tal que $\left(B^{\prime} \Vdash \varphi\left(\dot{x}_{G}\right)\right)^{\mathrm{L}[r, c]}$ ou $\left(B^{\prime} \Vdash \neg \varphi\left(\dot{x}_{G}\right)\right)^{\mathrm{L}[r, c]}$. Como $\varphi$ e $\neg \varphi$ são $\Sigma_{2}^{1}(r)$, podemos considerar apenas um caso, e o outro caso é inteiramente análogo. Assim, suponha que $\left(B^{\prime} \Vdash \varphi\left(x_{G}\right)\right)^{\mathrm{L}[r, c]}$. Sejam $M \preceq \mathrm{L}[r, c]$ um submodelo elementar enumerável contendo $B^{\prime}, r$ e $c$ e $C=\left\{x \in B^{\prime} \mid x\right.$ é $M$-genérico $\}$ condição $M$-master abaixo de $B^{\prime}$. Agora, em $V$, se $x \in C$, então $M[x] \models \varphi(x)$, pois $\left(C \Vdash \varphi\left(\dot{x}_{G}\right)\right)^{M}$, e como consequência da Absolutividade Analítica, $\varphi(x)$ é verdadeira em $V$ também. Assim, $C \subseteq A$. De forma completamente análoga, se $\left(B^{\prime} \Vdash \neg \varphi\left(\dot{x}_{G}\right)\right)^{\mathrm{L}[r, c]}$, então se obtém $A \cap C=\emptyset$.

Essa condição nos dá um método para obter um modelo onde todo conjunto $\boldsymbol{\Delta}_{2}^{1}$ de reais é $\mathbb{P}_{J}$-mensurável via forcing sobre $\mathrm{L}$, a saber, iterando o $\mathbb{P}_{J}$, partindo de $\mathrm{L}$ como modelo inicial (veja a Seção 5.4). Com isso, concluimos que a $\mathbb{P}_{J}$-mensurabilidade para os conjuntos $\boldsymbol{\Delta}_{2}^{1}$ nunca está na força de consistência de um cardinal inacessível. Analogamente, também é possível obter uma condição suficiente para que os conjuntos $\boldsymbol{\Sigma}_{2}^{1}$ de reais sejam $\mathbb{P}_{J}$-mensuráveis.

Teorema 5.3.5. Se para todo $r \in \omega^{\omega}$ o conjunto $\left\{x \in \omega^{\omega} \mid x\right.$ não é $\mathbb{P}_{J}$-genérico sobre $\left.\mathrm{L}[r]\right\}$ está em $\mathcal{N}_{J}$, então todos os conjuntos $\Sigma_{2}^{1}$ são $\mathbb{P}_{J}$-mensuráveis.

Demonstração. Sejam $A$ um conjunto $\Sigma_{2}^{1}$ e $r \in \omega^{\omega}$ um parâmetro real tal que $A$ é definível por uma fórmula $\varphi \in \Sigma_{2}^{1}(r)$. Seja $B$ um boreliano $J$-positivo com código de Borel $c$. $\operatorname{Em~} \mathrm{L}[r, c]$ existe $B^{\prime} \leq B$ tal que $\left(B^{\prime} \Vdash \varphi\left(\dot{x}_{G}\right)\right)^{\mathrm{L}[r, c]}$ ou $\left(B^{\prime} \Vdash \neg \varphi\left(\dot{x}_{G}\right)\right)^{\mathrm{L}[r, c]}$. Suponha o primeiro caso. Agora, em $V$, deve existir $C \leq B^{\prime}$ tal que $C$ é um conjunto formado por reais $\mathbb{P}_{J}$-genéricos sobre $\mathrm{L}[r, c]$, por hipótese. Assim, para todo $x \in C, \mathrm{~L}[r, c, x] \models \varphi(x)$, e pelo Teorema da Absolutividade de Shoenfield, o mesmo vale em $V$. Assim, $C \subseteq A$. Analogamente, se $\left(B^{\prime} \Vdash \neg \varphi\left(\dot{x}_{G}\right)\right)^{\mathrm{L}[r, c]}$, então $A \cap C=\emptyset$.

Diferentemente do caso dos conjuntos $\boldsymbol{\Delta}_{2}^{1}$, a $\mathbb{P}_{J}$-mensurabilidade para os conjuntos $\boldsymbol{\Sigma}_{2}^{1}$ não é possível usando uma simples iteração do forcing $\mathbb{P}_{J}$, pois precisamos adicionar um conjunto grande de genéricos sobre cada $\mathrm{L}[r]$. Em alguns casos, isso é possível, em outros casos, a $\mathbb{P}_{J}$-mensurabilidade implica que $\aleph_{1}$ é inacessível aos reais (veja a Seção 5.4).

Nosso objetivo agora é obter caracterizações dos tipos Solovay e Ihoda-Shelah. Como foi mencionado no início da seção, em geral a caracterização para a $\mathbb{P}_{J}$-mensurabilidade dos conjuntos $\boldsymbol{\Delta}_{2}^{1}$ e $\boldsymbol{\Sigma}_{2}^{1}$ não necessariamente envolve reais genéricos. Veremos que a caracterização para a mensurabilidade dessas classes é possível com o conceito de real quasi-genérico, que foi introduzido em 2005 [BHL05] por Brendle, Löwe e Halbeisen no contexto do forcing de Silver, e veremos que a noção de genérico e quasi-genérico coincidem quando o forcing tem ccc. 
Definição 5.3.6. Sejam $J$ um $\sigma$-ideal Borel gerado e $M$ um modelo transitivo de ZFC. Um real $x$ é dito $\mathbb{P}_{J}$-quasi-genérico sobre $M$ se para todo boreliano $B \in J$ codificado em $M$ tem-se $x \notin B$.

Lema 5.3.7. Sejam $J$ um $\sigma$-ideal Borel gerado tal que $\mathbb{P}_{J}$ tem ccc, e $M$ um modelo transitivo de $Z F C$. Então um real $x$ é $\mathbb{P}_{J}$-genérico se, e somente se, é $\mathbb{P}_{J}$-quasi-genérico sobre $M$.

Demonstração. Seja $x$ um real quasi-genérico sobre $M$. Para cada anticadeia maximal $E \in M$, seja $B_{E} \doteq \omega^{\omega} \backslash \bigcup E$. Como $\mathbb{P}_{J}$ tem ccc, $B_{E}$ é um boreliano codificado em $M$ tal que $B_{E} \in \mathcal{N}_{J}=J$. Como $x$ é quasi-genérico sobre $M$, então $x \notin B_{E}$, e portanto $x \in \bigcup E$. Segue que $x$ é $\mathbb{P}_{J}$-genérico sobre $M$.

Agora veremos que as recíprocas dos Teoremas 5.3.4 e 5.3.5 são verdadeiras quando trocamos $\mathbb{P}_{J^{-}}$ genéricos por $\mathbb{P}_{J}$-quasi-genéricos, com a ressalva de que o $\sigma$-ideal $J$ tenha complexidade no máximo $\boldsymbol{\Sigma}_{2}^{1}$. Esse resultado foi primeiramente demonstrado por Ikegami em 2010 [Ike10], no contexto dos forcings (fortemente) arbóreos, e por Khomskii em 2012 [K $\left.\mathrm{K}^{+} 12\right]$, para forcings idealizados. Além da relação entre transcendência sobre L e propriedades de regularidade, Ikegami em [Ike10] também investigou a relação desses com forcing absolutividade. Um exemplo clássico de caracterização para uma propriedade de regularidade via forcing absolutividade é o seguinte:

Teorema 5.3.8 ([Bag91, Woo82]). São equivalentes:

1. Toda fórmula $\Sigma_{3}^{1}$ é absoluta entre $\mathrm{V}$ e $\mathrm{V}[G]$, onde $G$ é um filtro $\mathbb{C}$-genérico.

2. Todo conjunto $\boldsymbol{\Delta}_{2}^{1}$ de reais é Baire mensurável.

Definição 5.3.9. Uma noção de forcing $\mathbb{P}$ é $\Sigma_{3}^{1}$-absoluta se toda fórmula $\Sigma_{3}^{1}$ é absoluta entre $\mathrm{V} e$ $\mathrm{V}[G]$, para todo $G$ filtro $\mathbb{P}$-genérico.

Antes de prosseguirmos, é importante esclarecer o que queremos dizer com "ideal $\Sigma_{2}^{1}$ ", uma vez que um $\sigma$-ideal é um subconjunto de $\mathcal{P}\left(\omega^{\omega}\right)$, e não de $\omega^{\omega}$.

Definição 5.3.10. Uma $\sigma$-ideal $J$ é dito $\boldsymbol{\Sigma}_{2}^{1}$ se $\left\{c \in \mathrm{BC} \mid B_{c} \in J\right\} \in \boldsymbol{\Sigma}_{2}^{1}$.

Sabemos que BC tem complexidade $\Pi_{1}^{1}$, então só é necessário limitar a complexidade de " $B_{c} \in J$ ", para um código $c \in \mathrm{BC}$.

Agora considere a seguinte definição, introduzida por Zapletal em 2008 [Zap08].

Definição 5.3.11. Sejam $J$ um $\sigma$-ideal Borel gerado tal que $\mathbb{P}_{J}$ é forcing próprio e $\Gamma$ uma classe projetiva qualquer.

- Sejam $B$ um boreliano $J$-positivo e $A \subseteq B \times \omega^{\omega}$ tal que $B=p A$. Dizemos que uma função $g: C \rightarrow \omega^{\omega}$ é uma uniformização boreliana de $A$ em relação a $B$ se $C$ é um boreliano $J$-positivo, $C \leq B, g$ é uma função boreliana $e$

$$
\forall x \in C((x, g(x)) \in A)
$$

- Dizemos que $\Gamma$ tem a propriedade de $\mathbb{P}_{J}$-uniformização se para todo $B \in \mathbb{P}_{J}$ e para todo $A \subseteq B \times \omega^{\omega}$ tal que $A \in \Gamma$, existe uma uniformização boreliana de $A$ em relação a $B$.

Zapletal provou em [Zap08] que $\boldsymbol{\Sigma}_{1}^{1}$ tem a propriedade de $\mathbb{P}_{J}$-uniformização, e que se $\mathrm{V}=\mathrm{L}$, então $\Pi_{1}^{1}$ não tem a propriedade de $\mathbb{P}_{J}$-uniformização. O Teorema de Ikegamii-Khomskii também mostra que a propriedade de $\mathbb{P}_{J}$-uniformização para $\boldsymbol{\Pi}_{1}^{1}$ pode ser caracterizada por forcing sobre $\mathrm{L}$, juntamente com a forcing absolutividade para as fórmulas $\Sigma_{3}^{1}$ e a $\mathbb{P}_{J}$-mensurabilidade dos conjuntos $\boldsymbol{\Delta}_{2}^{1}$. Um ingrediente crucial da prova do Teorema de Ikegami-Khomskii é o Teorema da Decomposição de Shoenfield (Teorema 2.3.3). Esse teorema tem como hipótese que $\aleph_{1}$ é acessível aos reais, e por isso lidaremos com o caso inacessível no lema seguinte. 
Lema 5.3.12. Se $\forall r\left(\aleph_{1}^{\mathrm{L}[r]}<\aleph_{1}\right)$, então todos os conjuntos $\boldsymbol{\Sigma}_{2}^{1}$ de reais são $\mathbb{P}_{J}$-mensuráveis.

Demonstração. Seja $r$ um real qualquer. Como $\aleph_{1}^{\mathrm{V}}$ é inacessível em $\mathrm{L}[r]$, a coleção de todos os densos em $\left(\mathbb{P}_{J}\right)^{\mathrm{L}[r]}$ é enumerável em V. Seja $\left\{D_{n} \mid n<\omega\right\}$ uma enumeração desses densos e para cada $n<\omega$, defina $A_{n} \doteq \omega^{\omega} \backslash \bigcup D_{n}$. Temos que $A_{n} \in \mathcal{N}_{J}$, para todo $n<\omega$ (esse argumento já apareceu na demonstração do Teorema 5.2.10). Assim, se $x$ não é $\mathbb{P}_{J}$-genérico sobre L $[r]$, então $x \notin \bigcup D_{n}$, para algum $D_{n}$, e então $x \in \bigcup_{n<\omega} A_{n} \in \mathcal{N}_{J}$, pois $\mathcal{N}_{J}$ é um $\sigma$-ideal. Provamos que

$$
\left\{x \in \omega^{\omega} \mid x \text { não é } \mathbb{P}_{J} \text {-genérico sobre } \mathrm{L}[r]\right\} \subseteq \bigcup_{n<\omega} A_{n} \in \mathcal{N}_{J} .
$$

Pelo Teorema 5.3.5, todos os conjuntos $\boldsymbol{\Sigma}_{2}^{1}$ são $\mathbb{P}_{J}$-mensuráveis.

Teorema 5.3.13 (Teorema de Ikegami-Khomskii [Ike10, $\left.\mathrm{K}^{+} 12\right]$ ). Seja $J$ um $\sigma$-ideal $\boldsymbol{\Sigma}_{2}^{1}$ Borel gerado tal que $\mathbb{P}_{J}$ é forcing próprio. São equivalentes:

1. Para todo $r \in \omega^{\omega}$ e para todo $B \in \mathbb{P}_{J}$, existe $x \in B$ que é $\mathbb{P}_{J}$-quasi-genérico sobre $\mathrm{L}[r]$.

2. Todos os conjuntos $\boldsymbol{\Delta}_{2}^{1}$ são $\mathbb{P}_{J}$-mensuráveis.

3. $\boldsymbol{\Pi}_{1}^{1}$ tem a propriedade de $\mathbb{P}_{J}$-uniformização.

4. $\mathbb{P}_{J}$ é $\Sigma_{3}^{1}$-absoluto.

\section{Demonstração.}

- $\left(1 . \Rightarrow 2\right.$.) Sejam $A$ um conjunto $\Delta_{2}^{1}$ e $r \in \omega^{\omega}$ um parâmetro real tal que $A \in \Delta_{2}^{1}(r)$. Seja $B$ um boreliano $J$-positivo.

Se, para todo $s \in \omega^{\omega}, \aleph_{1}^{\mathrm{L}[s]}<\aleph_{1}$, segue do Lema 5.3.12. Assim, suponha que existe $s \in \omega^{\omega}$ tal que $\aleph_{1}^{\mathrm{L}[s]}=\aleph_{1}$. Note que, nesse caso, $\aleph_{1}^{\mathrm{L}[r, s]}=\aleph_{1}$. Podemos codificar os reais $r, s$ em um único real $z$ tal que $\mathrm{L}[r, s]=\mathrm{L}[z]$ e $A$ é $\Delta_{2}^{1}(z)$.

Agora, tanto $B \cap A$ quanto $B \backslash A$ são conjuntos $\Sigma_{2}^{1}(z)$, pois $A$ é $\Delta_{2}^{1}(z)$. Assim, Pelo Teorema $\Sigma_{2}^{1}$ de Shoenfield, $A \cap B=\bigcup_{\alpha<\aleph_{1}} C_{\alpha}$ e $B \backslash A=\bigcup_{\beta<\aleph_{1}} D_{\beta}$, onde $C_{\alpha}, D_{\beta}$ são borelianos codificados em L $[z]$, para todos $\alpha, \beta<\aleph_{1}$.

Seja então $x \in B$ um real $\mathbb{P}_{J}$-quasi-genérico sobre $\mathrm{L}[z]$. Se $x \in A$, então existe $\alpha<\aleph_{1}$ tal que $x \in C_{\alpha} \subseteq A$, o que implica que $C_{\alpha}$ é um boreliano $J$-positivo. Se $x \notin A$, então existe $\beta<\aleph_{1}$ tal que $x \in D_{\beta} \subseteq B \backslash A$, e, portanto, $D_{\beta}$ é um boreliano $J$-positivo. Logo, $A$ é $\mathbb{P}_{J}$-mensurável.

- (2. $\Rightarrow$ 3.) Sejam $B$ um boreliano $J$-positivo e $A \subseteq B \times \omega^{\omega}$ um conjunto coanalítico. Pelo Teorema da Uniformização de Kondô, existe uma função $f$, cujo gráfico é $\Pi_{1}^{1}, \operatorname{dom}(f)=B$, e $f$ uniformiza $A$. Agora seja $D_{n, m} \doteq\left\{B^{\prime} \leq B \mid \forall x \in B^{\prime}(f(x)(n)=m)\right\}$ e considere $D_{n} \doteq \bigcup_{m<\omega} D_{n, m}$. Afirmamos que cada $D_{n}$ é denso abaixo de $B$.

De fato, fixe $B^{\prime} \leq B$ e $n<\omega$. Temos que para cada $m<\omega$ o conjunto $A_{m} \doteq\{x \in$ $\left.B^{\prime} \mid f(x)(n)=m\right\}$ é $\boldsymbol{\Delta}_{2}^{1}$ pois, como o gráfico de $f$ é $\boldsymbol{\Pi}_{1}^{1}$, então $A_{m}$ é $\boldsymbol{\Sigma}_{2}^{1}$ e, por outro lado, $\omega^{\omega} \backslash A_{m}=\bigcup_{k \neq m} A_{k}$. Assim, cada $A_{m}$ é $\mathbb{P}_{J}$-mensurável. Agora, como $B^{\prime}=\bigcup_{m<\omega} A_{m} \in \mathbb{P}_{J}$, então algum $A_{m} \notin \mathcal{N}_{J}$ pois $\mathcal{N}_{J}$ é um $\sigma$-ideal. Assim, como esse $A_{m}$ é $\mathbb{P}_{J}$-mensurável, deve existir $B^{\prime \prime} \in \mathbb{P}_{J}$ tal que $B^{\prime \prime} \subseteq A_{m} \subseteq B^{\prime}$ e, portanto, $B^{\prime \prime} \leq B^{\prime}$ e $B^{\prime \prime} \in D_{n, m}$.

Assim, seja $M$ um submodelo elementar enumerável contendo $B$ e $D_{n}$, para todo $n<\omega$. Seja $C=\{x \in B \mid x$ é $M$-genérico $\} \in \mathbb{P}_{J}$ condição $M$-master abaixo de $B$. Primeiro, note que se $m \neq k$ então $\bigcup\left(D_{n, m} \cap M\right)$ e $\bigcup\left(D_{n, k} \cap M\right)$ são disjuntos. Assim, definimos uma função $g: C \rightarrow \omega^{\omega}$ da seguinte forma:

$$
g(x)(n)=m \leftrightarrow x \in \bigcup\left(D_{n, m} \cap M\right) .
$$


Como cada $x \in C$ é $M$-genérico e cada $D_{n}$ é denso abaixo de $B$, então $x \in \bigcup\left(D_{n} \cap M\right)$. Logo, existe $m<\omega$ tal que $x \in \bigcup\left(D_{n, m} \cap M\right)$. Além disso, se $s \in \omega^{<\omega}$, então

$$
g^{-1}\left(\mathcal{O}_{s}\right)=\bigcap_{n \in \operatorname{dom}(s)} \bigcup\left(D_{n, s(n)} \cap M\right)
$$

e como $D_{n, m} \cap M$ é enumerável, para todos $n, m<\omega$ então $g^{-1}\left(\mathcal{O}_{s}\right)$ é um boreliano. Segue que $g$ é uma função boreliana.

Finalmente, note que $g=f \uparrow C$. Logo, $g$ é uma função boreliana que uniformiza $A$.

- (3. $\Rightarrow$ 4.) Sejam $r \in \omega^{\omega}$ um parâmetro real e considere a fórmula $\exists x \forall y \varphi(x, y) \in \Sigma_{3}^{1}(r)$ onde $\varphi \in$ $\Sigma_{1}^{1}(r)$. Vamos mostrar a absolutividade pra baixo. Isto é, suponha que $\mathrm{V}[G] \models \exists x \forall y \varphi(x, y)$ para algum filtro $\mathbb{P}_{J}$-genérico. Assim, sejam $B \in G$ uma condição e $\dot{x}$ um $\mathbb{P}_{J}$-nome tais que $B \Vdash \forall y \varphi(\dot{x}, y)$. Pelo Teorema 5.1.7, existem uma condição $B^{\prime} \leq B$ e função boreliana $f: B^{\prime} \rightarrow \omega^{\omega}$ tais que $B^{\prime} \Vdash \dot{x}=f\left(\dot{x}_{G}\right)$ e, portanto, $B^{\prime} \Vdash \forall y \varphi\left(f\left(\dot{x}_{G}\right), y\right)$. Vamos mostrar que a fórmula $\exists x \forall y \varphi(x, y)$ é verdadeira em $V$. Suponha que não. Então para todo $x \in B^{\prime}$ existe $y$ tal que $\neg \varphi(f(x), y)$. Usando a propriedade de $\mathbb{P}_{J}$-uniformização para o conjunto $A \doteq\left\{(x, y) \in B^{\prime} \times \omega^{\omega} \mid \neg \varphi(f(x), y)\right\} \in \Pi_{1}^{1}$, considere $C \leq B^{\prime}$ um boreliano e $g: C \rightarrow \omega^{\omega}$ função boreliana que uniformiza $A$. Assim, para todo $x \in C, \neg \varphi(f(x), g(x))$ vale em $V$. Por outro lado, como $f, g$ são funções borelianas, então a fórmula $\forall x \in C \neg \varphi(f(x), g(x))$ é $\Pi_{1}^{1}$, e por Absolutividade Analítica é verdadeira em $\mathrm{V}[G]$. Agora, se $C \in G$, então $x_{G} \in C^{\mathrm{V}[G]}$ e, portanto, $\mathrm{V}[G] \models \neg \varphi\left(f\left(x_{G}\right), g\left(x_{G}\right)\right)$. Segue que $C \Vdash \neg \varphi\left(f\left(\dot{x}_{G}\right), g\left(\dot{x}_{G}\right)\right)$, contradizendo $C \leq B^{\prime}$ e $B^{\prime} \Vdash \forall y \varphi\left(f\left(\dot{x}_{G}\right), y\right)$.

- (4. $\Rightarrow$ 1.) Sejam $r \in \omega^{\omega}$ e $B \in \mathbb{P}_{J}$ com código de Borel $c$. Como $J$ é $\Sigma_{2}^{1}$ então a fórmula

$$
\exists x\left(x \in B \wedge \mathrm{x} \text { é } \mathbb{P}_{J} \text {-quasi-genérico sobre } \mathrm{L}[r]\right)
$$

é $\Sigma_{3}^{1}(r, c)$.

Essa fórmula é verdadeira em qualquer extensão genérica $\mathrm{V}[G]$ tal que $B \in G$, pois $\mathbb{P}_{J}$ adiciona um real genérico (que pertence a todo boreliano $J$-positivo) sobre $V$ e, portanto, sobre $\mathrm{L}[r]$. Além disso, todo real $\mathbb{P}_{J}$-genérico sobre $\mathrm{L}[r]$ é também $\mathbb{P}_{J}$-quasi-genérico sobre $\mathrm{L}[r]$. Assim, essa fórmula também é verdadeira em $V$, por $\Sigma_{3}^{1}$-absolutividade.

Observação. Note que só usamos a hipótese de que o ideal é $\boldsymbol{\Sigma}_{2}^{1}$ na implicação $4 . \Rightarrow 1$. e, portanto, as implicações 1. $\Rightarrow 2$. $\Rightarrow 3 \Rightarrow 4$. seguem sem essa hipótese. Além disso, as afirmações 2., 3. e 4. são sempre equivalentes, não importando a complexidade do ideal $J$.

Teorema 5.3.14. Seja $J$ um $\sigma$-ideal Borel gerado $\boldsymbol{\Sigma}_{2}^{1}$ tal que $\mathbb{P}_{J}$ é forcing próprio. São equivalentes:

1. Todos os conjuntos $\boldsymbol{\Sigma}_{2}^{1}$ de reais são $\mathbb{P}_{J}$-mensuráveis.

2. Para todo $r \in \omega^{\omega}$, o conjunto $\left\{x \mid x\right.$ não é $\mathbb{P}_{J}$-quasi-genérico sobre $\left.\mathrm{L}[r]\right\} \in \mathcal{N}_{J}$.

\section{Demonstração.}

- $\left(1 . \Rightarrow 2\right.$.) Como $J$ é $\Sigma_{2}^{1}$, então o conjunto $\left\{x \mid x\right.$ não é $\mathbb{P}_{J}$-quasi-genérico sobre $\left.\mathrm{L}[r]\right\}$ é também $\Sigma_{2}^{1}$ e, portanto, $\mathbb{P}_{J}$-mensurável. Agora, suponha que esse conjunto não está em $\mathcal{N}_{J}$ e considere então $B \in \mathbb{P}_{J}$ contido em $\left\{x \mid x\right.$ não é $\mathbb{P}_{J}$-quasi-genérico sobre $\left.\mathrm{L}[r]\right\}$. Assim, para todo $x \in B$, $x$ não é $\mathbb{P}_{J}$-quasi-genérico sobre $\mathrm{L}[r]$. Logo, pelo Teorema 5.3 .13 , existe um conjunto $\boldsymbol{\Delta}_{2}^{1}$ que não é $\mathbb{P}_{J}$-mensurável. Absurdo. 
- (2. $\Rightarrow$ 1.) Sejam A um conjunto $\Sigma_{2}^{1}$ e $r \in \omega^{\omega}$ um parâmetro real tal que $A$ é $\Sigma_{2}^{1}(r)$. Se para todo $s \in \omega^{\omega}, \aleph_{1}^{\mathrm{L}[s]}<\aleph_{1}$, o resultado segue pelo Lema 5.3.12. Caso contrário, analisando a prova do Teorema 5.3.13, podemos supor sem perda de generalidade que $\aleph_{1}^{\mathrm{L}[r]}=\aleph_{1}$. Seja $B \in \mathbb{P}_{J}$ e considere o conjunto $A \cap B$. Se não existe um real $x \in A \cap B \mathbb{P}_{J}$-quasi-genérico sobre $\mathrm{L}[r]$, então, por hipótese

$$
A \cap B \subseteq\left\{x \mid x \text { não é } \mathbb{P}_{J} \text {-quasi-genérico sobre } \mathrm{L}[r]\right\} \in \mathcal{N}_{J} .
$$

Logo, existe $C \leq B$ tal que $A \cap C=\emptyset$. Caso contrário, usando o Teorema $\Sigma_{2}^{1}$ de Shoenfield, podemos escrever $A \cap B=\bigcup_{\alpha<\aleph_{1}} C_{\alpha}$ de modo que cada $C_{\alpha}$ é um boreliano codificado em

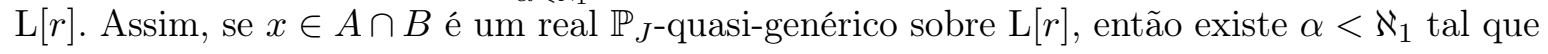
$x \in C_{\alpha} \subseteq A$ e $C_{\alpha}$ é $J$-positivo. Segue que $A$ é $\mathbb{P}_{J}$-mensurável.

Como o Teorema de Ikegami-Khomskii depende da complexidade dos ideais considerados, é natural perguntar quais dos $\sigma$-ideias considerados no capítulo anterior são $\boldsymbol{\Sigma}_{2}^{1}$. Além disso, juntando o Teorema 5.3.1 enunciado no início dessa seção com o Teorema de Ikegami-Khomskii, observamos que os reais novos sobre $\mathrm{L}[r]$ são os reais $\mathbb{S}$-quasi-genéricos, os reais ilimitados sobre $\mathrm{L}[r]$ são os reais $\mathbb{M}$-quasi-genéricos e os reais dominantes sobre $\mathrm{L}[r]$ são os reais $\mathbb{L}$-quasi-genéricos. Assim, é interessante também obter uma propriedade mais familiar para $\mathbb{P}_{J}$-quasi-genericidade. Faremos isso definindo a noção de $R$-transcendência para relações borelianas. Com isso, responderemos a pergunta do início do parágrafo para os casos dos forcings $\mathbb{S}, \mathbb{M}$ e $\mathbb{L}$.

Definição 5.3.15. Sejam $R$ uma relação boreliana em $\omega^{\omega} e A \subseteq \omega^{\omega}$. Sejam $y \in \omega^{\omega} e\left(y_{n}\right)_{n<\omega}$ uma sequência em $\omega^{\omega}$. Dizemos que

1. y é R-transcendente sobre $A$ se $\forall x \in A(x R y)$

2. $\left(y_{n}\right)_{n<\omega}$ é $R$ - $\sigma$-transcendente sobre $A$ se $\forall x \in A \exists n<\omega\left(x R y_{n}\right)$.

3. y é R-transcendente sobre $M$, onde $M$ é um modelo transitivo de $\mathrm{ZFC}$, se y é $R$-transcendente sobre $\omega^{\omega} \cap M$.

Definição 5.3.16. Seja $R$ uma relação boreliana em $\omega^{\omega}$. Definimos

1. Para cada $x \in \omega^{\omega}, K_{x}^{R} \doteq\left\{y \in \omega^{\omega} \mid \neg(x R y)\right\}$.

2. $J^{R}$ é o $\sigma$-ideal gerado por todos os $K_{x}^{R}$.

Trivialmente o $\sigma$-ideal $J^{R}$ é Borel gerado, pois a relação $R$ é boreliana. Além disso, $J^{R}$ é $\boldsymbol{\Sigma}_{2}^{1}$.

\section{Exemplo 5.3.17.}

1. Seja $R$ a relação definida por $x R y \leftrightarrow x \neq y$. Então $J^{R}=$ ctbl, cujo forcing correspondente é o forcing de Sacks.

2. Seja $R$ a relação definida por $x R y \leftrightarrow x \nsupseteq^{*} y$. Então $J^{R}=K_{\sigma}$, cujo forcing correspondente é o de Miller.

3. Seja $R$ a relação definida por $x R y \leftrightarrow y$ domina fortemente $x$. Então $J^{R}=\mathcal{L}^{0}$, cujo forcing correspondendo é o de Laver. 
Teorema 5.3.18. Sejam $R$ uma relação boreliana em $\omega^{\omega}$ e $M$ um modelo de ZFC tal que $\omega_{1} \subseteq M$.

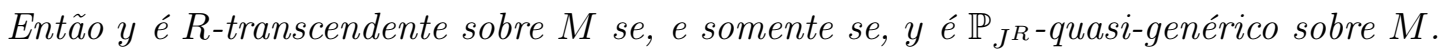

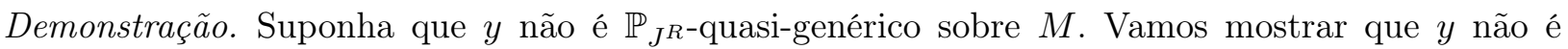
$R$-transcendente sobre $M$. Assim, seja $B \in J^{R}$ um boreliano codificado em $M$ tal que $y \in B$.

Usando a $\Sigma_{2}^{1}$-up-absolutividade, $M \models B \in J^{R}$, e então existe uma sequência $\left(x_{n}\right)_{n<\omega}$ em $M$ tal que $M=B \subseteq \bigcup_{n<\omega} K_{x_{n}}^{R}$. Agora, é fácil verificar que " $B \subseteq \bigcup_{n<\omega} K_{x_{n}}^{R}$ " pode ser escrito como uma fórmula $\Pi_{1}^{1}$. Portanto, por absolutividade analítica também vale em V. Como $y \in B$, então existe $n<\omega$ tal que $y \in K_{x_{n}}^{R}$, i.e., tal que $\neg\left(x_{n} R y\right)$. Logo, $y$ não é $R$-transcendente sobre $M$.

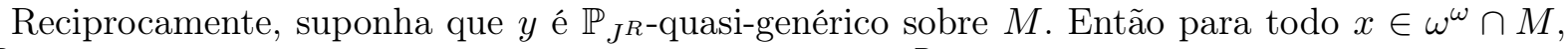
$K_{x}^{R} \in J$ é um boreliano codificado em $M$. Portanto $y \notin K_{x}^{R}$, e então $x R y$.

Com o teorema anterior e o Teorema de Ikegami-Khosmkii, as equivalências $n b \leftrightarrow n c$ do Teorema 5.3.1 ficam provadas, com $n \in\{1,2,3\}$.

Teorema 5.3.19. null é $\Pi_{1}^{1}$.

Demonstração. Seja $\left(s_{n}\right)_{n<\omega}$ uma enumeração recursiva de $\omega^{<\omega}$ tal que $\left|s_{n}\right| \leq n$, para todo $n<\omega$. Temos que $B \in$ null $\leftrightarrow \exists^{0} n\left(B \subseteq \mathcal{O}_{s_{n}} \wedge \mu\left(\mathcal{O}_{s_{n}}\right)<\frac{1}{n}\right)$, e a complexidade de " $B \subseteq \mathcal{O}_{s_{n}}$ " é $\Pi_{1}^{1}$.

O $\sigma$-ideal meag requer um argumento separado, mas não muito difícil. Para o $\sigma$-ideal $v^{0}$ dos conjuntos Silver-nulos (ou completamente doughnut-nulos), Ikegami em [Ike10] usou o seguinte resultado de Zapletal:

Teorema 5.3.20 ([Zap08]). Seja G um grafo em $2^{\omega}$ definido por

$$
x G y \leftrightarrow \exists ! n(x(n) \neq y(n)) .
$$

Seja $J_{G}$ o $\sigma$-ideal Borel gerado pelos conjuntos $G$-independentes, i.e., os borelianos $B$ de $2^{\omega}$ tais que dois elementos distintos $x, y \in B$ nunca estão $G$-conectados. Então para todo analítico A, ou $A \in J_{G}$ ou existe uma árvore de Silver $T$ tal que $[T] \subseteq A$.

Observando que $v^{0}$ e $J_{G}$ coincidem nos borelianos, é fácil verificar que " $B \in J_{G}$ " pode ser escrito usando uma fórmula $\Sigma_{2}^{1}$.

Teorema 5.3.21 ([Ike10]). $v^{0}$ é $\boldsymbol{\Sigma}_{2}^{1}$.

Embora a maioria dos exemplos de $\sigma$-ideais sejam $\boldsymbol{\Sigma}_{2}^{1}$, como os $\sigma$-ideais considerados em [Zap08], o $\sigma$-ideal $\mathcal{R}^{0}$ dos conjuntos completamente Ramsey-nulos é um contraexemplo. Isso foi provado por Sabok em 2012 [Sab12]. Isso nos leva naturalmente à seguinte pergunta.

Problema em aberto 4 . Se todo conjunto $\boldsymbol{\Delta}_{2}^{1}$ de reais é Ramsey mensurável, então para todo real $r$ existe um real $\mathbb{R}$-quasi-genérico sobre $\mathrm{L}[r]$ ?

\subsection{Considerações finais}

\section{Sobre o teorema de Brendle e Löwe 5.3.1}

No Teorema 5.3.1, foram provadas as equivalências $n a \leftrightarrow n b$, para $n \in\{1,2,3\}$. A equivalência com " $n a$ " decorre da análise específica de cada uma das noções de forcing em questão, como foi feito em [BL99]. Além das três noções de forcing $\mathbb{S}, \mathbb{M}$ e $\mathbb{L}$, Brendle e Löwe [BL99] também consideraram o forcing $\mathbb{C}$ de Cohen e o forcing $\mathbb{D}$ de Hechler. Brevemente,

Definição 5.4.1. O forcing de Hechler é o forcing $\mathbb{D} \doteq \omega \times \omega^{\omega}$ com a seguinte ordem:

$$
(n, f) \leq(m, g) \leftrightarrow n \geq m \wedge f \uparrow m=g \uparrow m \wedge f \geq g .
$$


É possível mostrar que esse forcing tem ccc. Agora, defina

$$
[n, f] \doteq\left\{x \in \omega^{\omega} \mid \forall n<\omega(f\lceil n \subseteq x \wedge x(n) \geq f(n))\} .\right.
$$

Da mesma forma que as condições do forcing de Cohen dão origem a abertos que formam a base canônica do espaço de Baire, os conjuntos da forma $[n, f]$, que são gerados pelas condições do forcing de Hechler, formam uma base para uma topologia em $\omega^{\omega}$, chamada de topologia dominante. Os forcings $\mathbb{C}$ e $\mathbb{D}$ são ditos forcings topológicos, enquanto que os forcings $\mathbb{S}, \mathbb{M}$ e $\mathbb{L}$ são ditos forcings não topológicos.

Temos uma noção natural de regularidade associada ao forcing de Hechler, a saber, a Baire mensurabilidade na topologia dominante. Assim, o forcing idealizado correspondente é o forcing dos borelianos da topologia dominante que não são magros na topologia dominante. Se um conjunto é Baire mensurável na topologia dominante, dizemos que esse conjunto é Hechler mensurável. Agora, a Hechler mensurabilidade para os conjuntos $\boldsymbol{\Sigma}_{2}^{1}$ de reais já está na força de consistência de um cardinal inacessível:

Teorema 5.4.2 ([BL99]). Todo conjunto $\boldsymbol{\Sigma}_{2}^{1}$ de reais é Hechler mensurável se, e somente se, $\forall r \in$ $\omega^{\omega}\left(\aleph_{1}^{\mathrm{L}}[r]<\aleph_{1}\right)$.

Aparentemente, há diferenças essenciais entre os forcings topológicos $\mathbb{C}, \mathbb{D}$ e os forcings não topológicos $\mathbb{S}, \mathbb{M}$ e $\mathbb{L}$, e por alguma razão, a mensurabilidade dos conjuntos $\boldsymbol{\Sigma}_{2}^{1}$ no caso dos forcings não topológicos é equivalente à mensurabilidade dos conjuntos $\boldsymbol{\Delta}_{2}^{1}$. Assim, a seguinte pergunta é natural:

Pergunta: Para forcings próprios da forma $\mathbb{P}_{J}$, que propriedades de $J$ são suficientes para que a $\mathbb{P}_{J}$-mensurabilidade dos conjuntos $\boldsymbol{\Sigma}_{2}^{1}$ seja equivalente à $\mathbb{P}_{J}$-mensurabilidade dos conjuntos $\boldsymbol{\Delta}_{2}^{1}$ ?

\section{Sobre resultados ótimos para a mensurabilidade}

Ao fim da Seção 4.1, foi mencionada a possibilidade "subir" na hierarquia projetiva, tentando obter mensurabilidade para classes projetivas cada vez mais complexas. Comentaremos brevemente a situação atual sobre os resultados ótimos para as noções de mensurabilidade que abordamos.

Como já foi dito, por um resultado de Shelah ([She84]), é possível construir somente na força de consistência de ZFC um modelo onde todos os conjuntos projetivos são Baire mensuráveis. Agora, pelo Corolário 3.5 de [BL99], se $\boldsymbol{\Gamma}$ é uma classe projetiva, então

$$
\Gamma(\mathbb{C}) \Rightarrow \Gamma(\mathbb{M}) \Rightarrow \Gamma(\mathbb{S}) .
$$

Com isso, a Miller mensurabilidade e a Marczewski mensurabilidade para todos os conjuntos projetivos de reais também são consistentes, na força de consistência de ZFC. No caso da Silver mensurabilidade, Brendle, Halbeisen e Löwe em 2005 [BHL05] provaram que todos os conjuntos projetivos de reais são Silver mensuráveis em um modelo obtido pelo $\aleph_{1}$-produto (com suporte finito) do forcing de Cohen.

Para o caso de Lebesgue mensurabilidade, o melhor resultado na força de consistência de ZFC é para os conjuntos $\boldsymbol{\Delta}_{3}^{1}$ (veja o Teorema 9.4.6 de [BJ]), e já foi dito que o caso $\boldsymbol{\Sigma}_{3}^{1}$ está na força de consistência de um inacessível, por um resultado de Shelah (veja [Rai84] para uma prova simplificada). Finalmente, para os casos da Ramsey mensurabilidade e da Laver mensurabilidade, os resultados ótimos não foram obtidos ainda. Resumimos esses comentários na tabela seguinte. 


\begin{tabular}{|c|c|}
\hline Regularidade & Resultado ótimo na hierarquia projetiva \\
\hline \hline Baire mensurabilidade & $\boldsymbol{\Sigma}_{n}^{1}$ para todo $n$ \\
\hline Miller mensurabilidade & $\boldsymbol{\Sigma}_{n}^{1}$ para todo $n$ \\
\hline Marczewski mensurabilidade & $\boldsymbol{\Sigma}_{n}^{1}$ para todo $n$ \\
\hline Silver mensurabilidade & $\boldsymbol{\Sigma}_{n}^{1}$ para todo $n$ \\
\hline Lebesgue mensurabilidade & $\boldsymbol{\Delta}_{3}^{1}$ \\
\hline Hechler mensurabilidade & $\boldsymbol{\Delta}_{2}^{1}$ \\
\hline Ramsey mensurabilidade & $?$ \\
\hline Laver mensurabilidade & $?$ \\
\hline
\end{tabular}

Problema em aberto 5. É consistente que todos os conjuntos projetivos de reais são Ramsey mensuráveis, na força de consistência de ZFC?

Problema em aberto 6. É consistente que todos os conjuntos projetivos de reais são Laver mensuráveis, na força de consistência de $\mathrm{ZFC}$ ?

\section{Sobre aplicações para o Teorema de Ikegami-Khomskii}

Para essa discussão, denotaremos REG para alguma propriedade de regularidade, $\boldsymbol{\Gamma}$ para alguma classe projetiva e $\boldsymbol{\Gamma}(\mathrm{REG})$ é a afirmação

"Todo conjunto de reais em $\boldsymbol{\Gamma}$ tem a propriedade de regularidade REG."

A primeira observação sobre os teoremas da Seção 5.3, especialmente o Teorema de IkegamiKhomskii 5.3.13, é que questões sobre uma noção de regularidade se transformam em questões sobre a noção de forcing relacionada com essa noção de regularidade. Consequentemente, perguntas sobre relações entre noções de regularidade, se transformam em perguntas sobre relações entre noções de forcing.

Exemplo 5.4.3. Como o forcing de Cohen adiciona reais de Cohen mas não adiciona reais aleatórios, e essa propriedade do forcing de Cohen é preservada por iteração com suporte finito, uma iteração do forcing de Cohen partindo de $\mathrm{L}$ nos dá um modelo de ZFC onde $\boldsymbol{\Delta}_{2}^{1}$ (Baire) $+\neg \boldsymbol{\Delta}_{2}^{1}$ (Lebesgue). Reciprocamente, como o forcing aleatório adiciona reais aleatórios mas não adiciona reais de Cohen, e essa propriedade do forcing aleatório é preservada por iteração com suporte finito, uma iteração do forcing de Cohen partindo de L nos dá um modelo de ZFC onde $\boldsymbol{\Delta}_{2}^{1}$ (Lebesgue) $+\neg \boldsymbol{\Delta}_{2}^{1}$ (Baire).

Exemplo 5.4.4. O forcing de Laver adiciona reais dominantes sobre o modelo inicial, que são ilimitados também sobre o modelo inicial. O forcing de Miller adiciona reais que são ilimitados sobre o modelo inicial. Assim, se temos $\boldsymbol{\Delta}_{2}^{1}$ (Laver), o que é possivel em uma iteração do forcing de Laver a partir de L (com suporte enumerável), também temos $\boldsymbol{\Delta}_{2}^{1}(\mathrm{Miller})$. O mesmo vale para a classe $\boldsymbol{\Sigma}_{2}^{1}$ por causa da equivalência com $\boldsymbol{\Delta}_{2}^{1}$ para esses forcings. Por outro lado, o forcing de Miller adiciona reais ilimitados, mas não adiciona reais dominantes sobre o modelo inicial, e essa propriedade é preservada por iteração (com suporte enumerável). Assim, uma iteração do forcing de Miller nos dá um modelo de ZFC onde $\boldsymbol{\Sigma}_{2}^{1}$ (Miller) $+\neg \boldsymbol{\Sigma}_{2}^{1}$ (Laver).

Quais tipos de reais uma noção de forcing adiciona, ou deixa de adicionar, em um processo iterativo, é o que permite o estudo das relações entre noções de regularidade. Portanto, para explorar as relações de implicação entre noções de regularidade, ou a independência entre cada uma delas, o ambiente adequado é a teoria do forcing iterado. Para esse assunto, textos excelentes são [BJ] e [Hal12]. 


\section{Referências Bibliográficas}

[Ale16] Paul Alexandroff. Sur la puissance des ensembles mesurables b 162. 1916. 13

[Bag91] Juan Pigrau Bagaria. Definable forcing and regularity properties of projective sets of reals. University of California, Berkeley, 1991. 96

[Bai99] René Baire. Sur les fonctions de variables réelles. Annali di Matematica Pura ed Applicata (1898-1922), 3(1):1-123, 1899. 10

[Ben83] Ivar Bendixson. Quelques theorèmes de la théorie des ensembles de points. Acta Mathematica, 2(1):415-429, 1883. 3

[Ber07] Felix Bernstein. Zur theorie der trigonometrischen reihe. Journal für die reine und angewandte Mathematik, 132:270-278, 1907. 12

[BHL05] Jörg Brendle, Lorenz Halbeisen, and Benedikt Löwe. Silver measurability and its relation to other regularity properties. In Mathematical Proceedings of the Cambridge Philosophical Society, volume 138, pages 135-149. Cambridge University Press, 2005. 95, 101

[BJ] Tomek Bartoszyñski and Haim Judah. Set theory: on the structure of the real line, ak peters, 1995. MR1350295 (96k: 03002). 73, 101, 102

[BL99] Jörg Brendle and Benedikt Löwe. Solovay-type characterizations for forcing-algebras. The Journal of Symbolic Logic, 64(3):1307-1323, 1999. 61, 93, 100, 101

[Bor98] Émile Borel. Leçons sur la théorie des fonctions. Gauthier-Villars et fils, 1898. 10

[Bor19] Emil Borel. Sur la classification des ensembles de mesure nulle. Bull. Soc. Math. France, 47:97-125, 1919. 72

[Bre94] Jörg Brendle. Strolling through paradise. arXiv preprint math/9407204, 1994. 61

$\left[\mathrm{C}^{+} 02\right]$ Paul Cohen et al. The discovery of forcing. Rocky Mountain Journal of Mathematics, 32(4):1071-1100, 2002. 41, 48, 49

[Can72] Georg Cantor. Über die ausdehnung eines satzes aus der theorie der trigonometrischen reihen. Mathematische Annalen, 5(1):123-132, 1872. 1

[Can74] Georg Cantor. Ueber eine eigenschaft des inbegriffs aller reellen algebraischen zahlen. Journal für die reine und angewandte Mathematik, 77:258-262, 1874. 2

[Can77] Georg Cantor. Ein beitrag zur mannigfaltigkeitslehre. Journal für die reine und angewandte Mathematik, 84:242-258, 1877. 2

[Can83] Georg Cantor. Grundlagen einer allgemeinen Mannichfaltigkeitslehre: ein mathematischphilosophischer Versuch in der Lehre des Unendlichen. Teubner, 1883. 2

[Can84] Georg Cantor. De la puissance des ensembles parfaits de points. Acta Mathematica, 4(1):381-392, 1884. 3 
[Can91] Georg Cantor. Uber eine elementare frage der mannigfaltigkeitslehre. Jahresbericht der Deutschen Mathematiker-Vereinigung, 1(1):75-78, 1891. 3

[Coh08] Paul J Cohen. Set theory and the continuum hypothesis. Courier Corporation, 2008. 39

[Dav64] Morton Davis. Infinite games of perfect information. Advances in game theory, 52:85-101, 1964. 37

[DPH00] Carlos A Di Prisco and James M Henle. Doughnuts, floating ordinals, square brackets, and ultraflitters. The Journal of Symbolic Logic, 65(1):461-473, 2000. 78

[El174] Erik Ellentuck. A new proof that analytic sets are ramsey. Journal of Symbolic Logic, pages $163-165,1974.76,77,78$

[ER52] Paul Erdos and Richard Rado. Combinatorial theorems on classifications of subsets of a given set. Proceedings of the London mathematical Society, 3(1):417-439, 1952. 75

[Fra22] Adolf Fraenkel. Zu den grundlagen der cantor-zermeloschen mengenlehre. Mathematische annalen, 86(3):230-237, 1922. 3

[Fri73] Harvey M Friedman. Borel sets and hyperdegrees1. The Journal of Symbolic Logic, 38(3):405-409, 1973. 69

[Göd38] Kurt Gödel. The consistency of the axiom of choice and of the generalized continuumhypothesis. Proceedings of the National Academy of Sciences, 24(12):556-557, 1938. 14, 18

[GP73] Fred Galvin and Karel Prikry. Borel sets and ramsey's theorem 1. The Journal of Symbolic Logic, 38(2):193-198, 1973. 75

[GRSS95] Martin Goldstern, Miroslav Repickỳ, Saharon Shelah, and Otmar Spinas. On tree ideals. Proceedings of the American Mathematical Society, 123(5):1573-1581, 1995. 73, 74

[GS53] David Gale and Frank M Stewart. Infinite games with perfect information. Contributions to the Theory of Games, 2:245-266, 1953. 34, 35, 36

[Haj56] András Hajnal. On a consistency theorem connected with the generalized continuum problem. Mathematical Logic Quarterly, 2(8-9):131-136, 1956. 19

[Hal03] Lorenz Halbeisen. Making doughnuts of cohen reals. Mathematical Logic Quarterly, 49(2):173-178, 2003. 79, 80, 81

[Hal12] Lorenz J Halbeisen. Combinatorial set theory. Springer, 2012. 102

[Hau14] F Hausdorff. Grundzuge der mengenlehre. reprinted (1949) by the chelsea publishing company. New York, 1914. 11

[Ike10] Daisuke Ikegami. Forcing absoluteness and regularity properties. Annals of Pure and Applied Logic, 161(7):879-894, 2010. 67, 96, 97, 100

[IS89] Jaime I Ihoda and Saharon Shelah. $\delta 12$-sets of reals. Annals of Pure and Applied Logic, $42(3): 207-223,1989.65$

[Jec03] Thomas Jech. Set theory. the third millennium edition. Springer Monographs in Mathematics. Springer-Verlag, 2003. 19, 73

[Jef13] Thomas Jefferson. Letter to isaac mcpherson. 1813. 39 
$\left[\mathrm{K}^{+} 12\right] \quad$ Yurii Daniilovich Khomskii et al. Regularity properties and definability in the real number continuum: idealized forcing, polarized partitions, Hausdorff gaps and mad families in the projective hierarchy. ILLC, 2012. 61, 96, 97

[Kan08] Akihiro Kanamori. The higher infinite: large cardinals in set theory from their beginnings. Springer Science \& Business Media, 2008. 19, 36, 37, 38, 88

[Kan16] Akihiro Kanamori. Mathias and set theory. Mathematical Logic Quarterly, 62(3):278294, 2016. 75

[Kec77] Alexander S Kechris. On a notion of smallness for subsets of the baire space. Transactions of the American Mathematical Society, 229:191-207, 1977. 69, 70

[Kec95] Alexander S Kechris. Classical descriptive set theory. Graduate Texts in Mathematics, 156, 1995. 38

[Kle43] Stephen Cole Kleene. Recursive predicates and quantifiers. Transactions of the American Mathematical Society, 53(1):41-73, 1943. 15

[Kle55a] Stephen C Kleene. Arithmetical predicates and function quantifiers. Transactions of the American Mathematical Society, 79(2):312-340, 1955. 15

[Kle55b] Stephen Cole Kleene. Hierarchies of number-theoretic predicates. Bulletin of the American Mathematical Society, 61(3):193-213, 1955. 15

[Kle55c] Stephen Cole Kleene. On the forms of the predicates in the theory of constructive ordinals (second paper). American journal of mathematics, 77(3):405-428, 1955. 15

[Kon37] Motokiti Kondô. L'uniformisation des complémentaires analytiques. Proceedings of the Imperial Academy, 13(8):287-291, 1937. 14, 15

[Kun14] Kenneth Kunen. Set theory an introduction to independence proofs, volume 102. Elsevier, 2014. $7,19,40$

$\left[\mathrm{L}^{+} 25\right] \quad$ Nicolas Luzin et al. Sur les ensembles projectifs de m. henri lebesgue. CR Hebdomadaires des Seances Acad. Sci. Paris, 180:1572-1574, 1925. 13, 14

[Lav76] Richard Laver. On the consistency of borel's conjecture. Acta mathematica, 137(1):151169, 1976. 59, 72, 73

[Leb02] Henri Lebesgue. Intégrale, longueur, aire. Annali di Matematica Pura ed Applicata (1898-1922), 7(1):231-359, 1902. 11

[Leb05] Henri Lebesgue. Sur les fonctions représentables analytiquement. Journal de mathematiques pures et appliquees, 1:139-216, 1905. 11, 12, 13

[Lév60a] Azriel Lévy. Axiom schemata of strong infinity in axiomatic set theory. Pacific journal of mathematics, 10(1):223-238, 1960. 19, 40

[Lev60b] Azriel Levy. Principles of reflection in axiomatic set theory. Fundamenta Mathematicae, 1(49):1-10, 1960. 40

[Lev63] Azriel Levy. Independence results in set theory by cohen's method iv. Notices of the American Mathematical Society, 10:592-593, 1963. 88

[Lev02] Azriel Levy. Basic set theory, volume 13. Courier Corporation, 2002. 1

[LS18] Nicolas Lusin and Wacław Sierpínski. Sur quelques proprietes des ensembles (A). Imprimerie de l'Universite, 1918. 14 
[LS23] Nicolas Lusin and Wacław Sierpínski. Sur une ensemble non mesurable b. Journal de Mathématiques Pures et Appliquées, 2(9):53-72, 1923. 14, 26, 27, 29

[Lus26] N Lusin. Mémoire sur les ensembles analytiques et projectifs. Mathematicheskii Sbornii, 33(3):237-290, 1926. 14

[Luz17] Nicolas Luzin. Sur la classification de m. baire. CR Acad. Sci. Paris, 164:91-94, 1917. 13,14

[Mar75] Donald A Martin. Borel determinacy. Annals of Mathematics, pages 363-371, 1975. 38

[Mat69] Adrian Richard David Mathias. On a generalization of Ramsey's theorem. PhD thesis, University of Cambridge, 1969. 76

[Mau81] R Daniel Mauldin. The Scottish book: mathematics from the Scottish Café. Birkhauser, 1981. 36, 37

[Mil84] A Miller. Rational perfect set forcing. Contemporary Mathematics, 31:143-159, 1984. 70

[Mon56] Richard M Montague. Zermelo-fraenkel set theory is not a finite extension of zermelo set theory. Bulletin of the American Mathematical Society, 62:260, 1956. 40

[Mon61] Richard Montague. Fraenkel's addition to the axioms of zermelo. 1961. 40

[MS62] Jan Mycielski and Hugo Steinhaus. A mathematical axiom contradicting the axiom of choice. Bulletin de l'Académie Polonaise des Sciences, 10:1-3, 1962. 36

[MŚ64] Jan Mycielski and Stanisław Świerczkowski. On the lebesgue measurability and the axiom of determinateness. Fundamenta Mathematicae, 54(1):67-71, 1964. 38

[Oxt13] John C Oxtoby. Measure and category: A survey of the analogies between topological and measure spaces, volume 2. Springer Science \& Business Media, 2013. 12

[Pri68] Karel Prikry. Changing measurable into accessible cardinals(changing measurable into accessible cardinals). 1968. 76

[Rai84] Jean Raisonnier. A mathematical proof of s. shelah's theorem on the measure problem and related results. Israel Journal of Mathematics, 48(1):48-56, 1984. 101

[Ram30] FP Ramsey. On a problem of formal logic. Proceedings of the London Mathematical Society, 2(1):264-264, 1930. 75

[Sab12] Marcin Sabok. Complexity of ramsey null sets. Advances in Mathematics, 230(3):11841195, 2012. 100

[Sac71] Gerald E Sacks. Forcing with perfect closed sets. In Axiomatic set theory, volume 1, pages 331-355. Proceedings of Symposia in Pure Mathematics, 1971. 67

[She80] Saharon Shelah. Independence results. The Journal of Symbolic Logic, 45(3):563-573, 1980. 58,59

[She82] Saharon Shelah. Proper forcing. In Proper Forcing, pages 73-113. Springer, 1982. 59

[She84] Saharon Shelah. Can you take solovay's inaccessible away? Israel Journal of mathematics, 48(1):1-47, 1984. 62, 101

[Sho61] J Shoenfield. The problem of predicativity in: Y. barmhillel, eij poznanski, m. o. rabin and a. robinson, eds., essays on the foundations of mathematics dedicated to aa fraenkel on his seventieth anniversary, 1961. 31, 33 
[Sho71] Joseph R Shoenfield. Unramified forcing. In Axiomatic set theory, volume 13, pages 357-381. AMS Providence, RI, 1971. 43

[Sie25] Wacław Sierpiński. Sur une classe d'ensembles. Fundamenta Mathematicae, 1(7):237243, 1925. 13, 14, 31

[Sie28] Wacław Sierpiński. Sur un ensemble non dénombrable, dont toute image continue est de mesure nulle. Fundamenta Mathematicae, 1(11):302-303, 1928. 72

[Sil70] Jack Silver. Every analytic set is ramsey. The Journal of Symbolic Logic, 35(1):60-64, 1970. 76

[Sko22] Thoralf Skolem. Einige bemerkungen zur axiomatischen begründung der mengenlehre. 1922. 4

[Sko34] Th Skolem. Über die nicht-charakterisierbarkeit der zahlenreihe mittels endlich oder abzählbar unendlich vieler aussagen mit ausschliesslich zahlenvariablen. Fundamenta mathematicae, 23(1):150-161, 1934. 4

[Sol69] Robert M Solovay. On the cardinality of $\backslash$ sum_2^ 1 sets of reals. In Foundations of Mathematics, pages 58-73. Springer, 1969. 66

[Sol70] Robert M Solovay. A model of set-theory in which every set of reals is lebesgue measurable. Annals of Mathematics, pages 1-56, 1970. 23, 61, 62, 63, 83

[Sus17] Mikhail Suslin. Sur une définition des ensembles mesurables b sans nombres transfinis. CR Acad. Sci. Paris, 164(2):88-91, 1917. 13, 27

[Szp35] Edward Szpilrajn. Sur une classe de fonctions de m. sierpiński et la classe correspondante d'ensembles. Fundamenta Mathematicae, 24(1):17-34, 1935. 66

[Vit05] Giuseppe Vitali. Sul problema della misura dei Gruppi di punti di una retta: Nota. Tip. Gamberini e Parmeggiani, 1905. 12

[VN23] John Von Neumann. Zur einführung der transfiniten zahlen. Acta Szeged, 1:199-208, 1923. 4

[VN29] John Von Neumann. Über eine widerspruchsfreiheit frage in der axiomatischen mengenlehre. Journal für die reine und angewandte Mathematik, 160:227-241, 1929. 4

[Wea14] Nik Weaver. Forcing for mathematicians. World Scientific, 2014. 40

[Woo82] W Hugh Woodin. On the consistency strength of projective uniformization. In Studies in Logic and the Foundations of Mathematics, volume 107, pages 365-384. Elsevier, 1982. 96

[Zap04] Jindřich Zapletal. Descriptive set theory and definable forcing. Number 793. American Mathematical Soc., 2004. 61, 83, 85

[Zap08] Jindřich Zapletal. Forcing idealized, volume 174. Cambridge University Press Cambridge, 2008. $85,96,100$

[Zer04] Ernst Zermelo. Beweis, daß jede menge wohlgeordnet werden kann. Mathematische Annalen, 59(4):514-516, 1904. 3

[Zer07] Ernst Zermelo. Neuer beweis für die möglichkeit einer wohlordnung. Mathematische Annalen, 65(1):107-128, 1907. 3

[Zer08] Ernst Zermelo. Untersuchungen über die grundlagen der mengenlehre. i. Mathematische Annalen, 65(2):261-281, 1908. 3 